\title{
Second-Order Relative Motion Equations
}

\author{
Christopher D. Karlgaard
}

Thesis submitted to the Faculty of the

Virginia Polytechnic Institute and State University

in partial fulfillment of the requirements for the degree of

\author{
Master of Science \\ in \\ Aerospace Engineering
}

Dr. Frederick H. Lutze - chair

Dr. Eugene M. Cliff

Dr. Christopher D. Hall

July 2001

Blacksburg, Virginia

Keywords: Orbital Mechanics, Perturbation Methods

Copyright 2001, Christopher D. Karlgaard 


\title{
Second-Order Relative Motion Equations
}

\author{
Christopher D. Karlgaard
}

(ABSTRACT)

This thesis presents an approximate solution of second order relative motion equations. The equations of motion for a Keplerian orbit in spherical coordinates are expanded in Taylor series form using reference conditions consistent with that of a circular orbit. Only terms that are linear or quadratic in state variables are kept in the expansion. A perturbation method is employed to obtain an approximate solution of the resulting nonlinear differential equations. This new solution is compared with the previously known solution of the linear case to show improvement, and with numerical integration of the quadratic differential equation to understand the error incurred by the approximation. In all cases, the comparison is made by computing the difference of the approximate state (analytical or numerical) from numerical integration of the full nonlinear Keplerian equations of motion. 


\section{Acknowledgments}

First I would like to thank my advisor, Fred Lutze, for suggesting this topic to me and guiding me through its completion. I had lots of ideas during the course of this project, and he was patient with me when I was wrong and encouraged me when they worked out. I enjoyed all the discussions we had about this and other "neat" dynamics problems.

Thanks to Chris Hall for his support of this project, for helping me learn $\mathrm{HT}_{\mathrm{E} X} \mathrm{X}$ and for serving on my committee. I also thank Eugene Cliff for taking the time from his schedule to serve on my committee and review the final draft.

Thanks for all my cohorts down here in Blacksburg for making these years fun and exciting, and thanks to all my friends back home who don't get to see me much anymore just for being there.

Finally, thanks to my family for everything. 


\section{Contents}

1 Introduction $\quad 1$

1.1 Problem Statement . . . . . . . . . . . . . . . . . . . 1

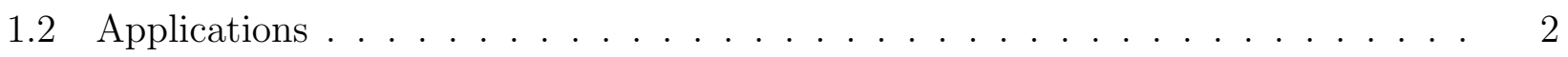

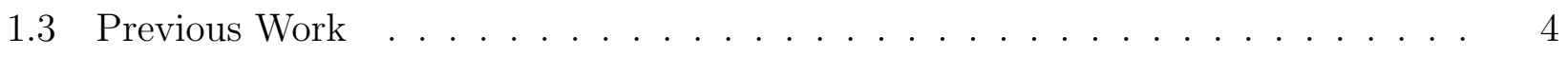

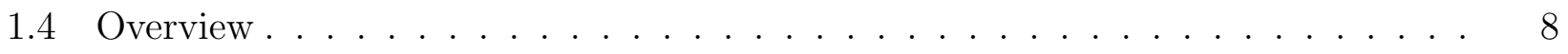

2 Preliminaries $\quad 9$

2.1 Orbital Mechanics . . . . . . . . . . . . . . . . . . . . . . . . 9

2.1.1 The Two-Body Problem ........................ 9

2.1.2 Equations of Motion ..................... 11

2.2 Perturbation Methods . . . . . . . . . . . . . . . . . 13

2.2.1 The Straightforward Expansion . . . . . . . . . . . . . . . . 14

2.2.2 The Method of Multiple Scales . . . . . . . . . . . . . . . 16

3 Linear Model $\quad 23$

3.1 Equations of Motion . . . . . . . . . . . . . . . . . . 23

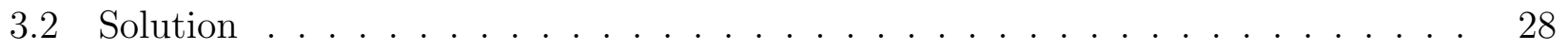

3.3 Relative Position and Velocity Vectors . . . . . . . . . . . . . . . . . 31

4 Nonlinear Model $\quad 34$

4.1 Equations of Motion . . . . . . . . . . . . . . . . . . . . . . 34

4.2 The Method of Multiple Scales . . . . . . . . . . . . . . . . . 37

4.2.1 First Order Solution . . . . . . . . . . . . . . . . . . 40 
4.2 .2 Second Order Solution . . . . . . . . . . . . . . . . 46

4.2 .3 Solvability Conditions . . . . . . . . . . . . . . . . . 51

4.2 .4 Overall Solution . . . . . . . . . . . . . . . . . 54

4.3 Summary . . . . . . . . . . . . . . . . . . . . . 60

$\begin{array}{llr}5 & \text { Results } & 61\end{array}$

6 Conclusions $\quad 63$

$\begin{array}{lr}\text { A Nonlinear Model } & 65\end{array}$

B Method of Multiple Scales $\quad 72$

B.1 Evaluation of the First Order PDE . . . . . . . . . . . . . . . . . . . 72

B.2 Evaluation of the Second Order PDE . . . . . . . . . . . . . . . . . . . 74

$\begin{array}{lr}\text { C Plots } & 84\end{array}$ 


\section{List of Figures}

1.1 Geometry of the Relative Motion Problem . . . . . . . . . . . . . . 2

2.1 Orbit with Constant Out-of-Plane Offset . . . . . . . . . . . . . . . 11

2.2 Spherical Coordinate System . . . . . . . . . . . . . . . . . . . . . . . . 12

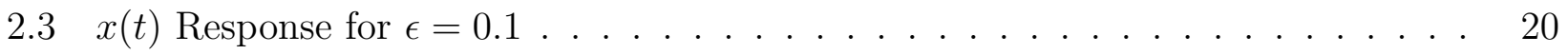

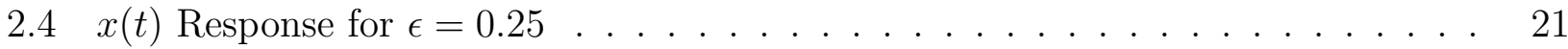

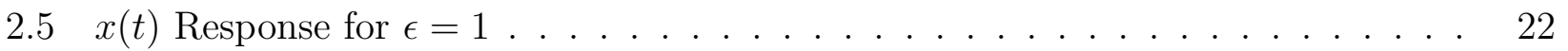

3.1 Relative Spherical Coordinate System . . . . . . . . . . . . . . . . . . . . 32

C.1 Error of Position Variables for $\Delta \hat{r}(0)=0.001 \ldots \ldots \ldots \ldots \ldots$

C.2 Error of Velocity Variables for $\Delta \hat{r}(0)=0.001 \ldots \ldots \ldots \ldots$

C.3 Magnitude of Relative Position and Velocity Error for $\Delta \hat{r}(0)=0.001 \ldots \ldots$. 87

C.4 Error of Position Variables for $\Delta \hat{\phi}(0)=0.001 \ldots \ldots \ldots$

C.5 Error of Velocity Variables for $\Delta \hat{\phi}(0)=0.001 \ldots \ldots \ldots \ldots$

C.6 Magnitude of Relative Position and Velocity Error for $\Delta \hat{\phi}(0)=0.001 \ldots \ldots$

C.7 Error of Position Variables for $\Delta \hat{v}_{r}(0)=0.001 \ldots \ldots \ldots \ldots \ldots$

C.8 Error of Velocity Variables for $\Delta \hat{v}_{r}(0)=0.001 \ldots \ldots \ldots \ldots$

C.9 Magnitude of Relative Position and Velocity Error for $\Delta \hat{v}_{r}(0)=0.001 \ldots \ldots . \quad 93$

C.10 Error of Position Variables for $\Delta \hat{\omega}_{\theta}(0)=0.001 \ldots \ldots \ldots \ldots$

C.11 Error of Velocity Variables for $\Delta \hat{\omega}_{\theta}(0)=0.001 \ldots \ldots \ldots \ldots$

C.12 Magnitude of Relative Position and Velocity Error for $\Delta \hat{\omega}_{\theta}(0)=0.001 \ldots \ldots .96$

C.13 Error of Position Variables for $\Delta \hat{\omega}_{\phi}(0)=0.001 \ldots \ldots \ldots \ldots \ldots$. . . . . 97

C.14 Error of Velocity Variables for $\Delta \hat{\omega}_{\phi}(0)=0.001 \ldots \ldots \ldots \ldots$ 
C.15 Magnitude of Relative Position and Velocity Error for $\Delta \hat{\omega}_{\phi}(0)=0.001$. . . . . 99

C.16 Error of Position Variables for $\Delta \hat{r}(0)=0.001, \Delta \hat{\phi}(0)=0.001 \ldots$. . . . . . 100

C.17 Error of Velocity Variables for $\Delta \hat{r}(0)=0.001, \Delta \hat{\phi}(0)=0.001 \ldots$. . . . . . . 101

C.18 Magnitude of Relative Position and Velocity Error for $\Delta \hat{r}(0)=0.001, \Delta \hat{\phi}(0)=0.001102$

C.19 Error of Position Variables for $\Delta \hat{v}_{r}(0)=0.001, \Delta \hat{\phi}(0)=0.001 \ldots \ldots 3$

C.20 Error of Velocity Variables for $\Delta \hat{v}_{r}(0)=0.001, \Delta \hat{\phi}(0)=0.001$. . . . . . . . 104

C.21 Magnitude of Relative Position and Velocity Error for $\Delta \hat{v}_{r}(0)=0.001, \Delta \hat{\phi}(0)=$

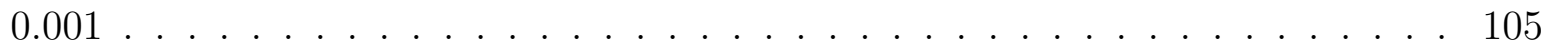

C.22 Error of Position Variables for $\Delta \hat{\omega}_{\theta}(0)=0.001, \Delta \hat{\phi}(0)=0.001 \ldots$. . . . . . 106

C.23 Error of Velocity Variables for $\Delta \hat{\omega}_{\theta}(0)=0.001, \Delta \hat{\phi}(0)=0.001 \ldots$. . . . . . 107

C.24 Magnitude of Relative Position and Velocity Error for $\Delta \hat{\omega}_{\theta}(0)=0.001, \Delta \hat{\phi}(0)=$

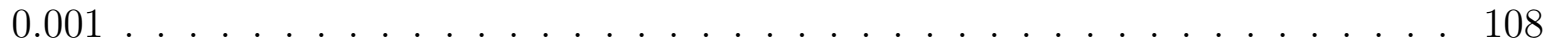

C.25 Error of Position Variables for $\Delta \hat{r}(0)=0.001, \Delta \hat{\omega}_{\phi}(0)=0.001 \ldots$. . . . . . 109

C.26 Error of Velocity Variables for $\Delta \hat{r}(0)=0.001, \Delta \hat{\omega}_{\phi}(0)=0.001$. . . . . . . . 110

C.27 Magnitude of Relative Position and Velocity Error for $\Delta \hat{r}(0)=0.001, \Delta \hat{\omega}_{\phi}(0)=$ $0.001 \ldots \ldots \ldots \ldots \ldots \ldots \ldots \ldots \ldots$

C.28 Error of Position Variables for $\Delta \hat{v}_{r}(0)=0.001, \Delta \hat{\omega}_{\phi}(0)=0.001$. . . . . . . . 112

C.29 Error of Velocity Variables for $\Delta \hat{v}_{r}(0)=0.001, \Delta \hat{\omega}_{\phi}(0)=0.001 \ldots \ldots$. . . . 113

C.30 Magnitude of Relative Position and Velocity Error for $\Delta \hat{v}_{r}(0)=0.001, \Delta \hat{\omega}_{\phi}(0)=$

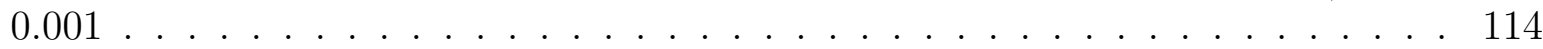

C.31 Error of Position Variables for $\Delta \hat{\omega}_{\theta}(0)=0.001, \Delta \hat{\omega}_{\phi}(0)=0.001 \ldots \ldots$

C.32 Error of Velocity Variables for $\Delta \hat{\omega}_{\theta}(0)=0.001, \Delta \hat{\omega}_{\phi}(0)=0.001 \ldots \ldots$

C.33 Magnitude of Relative Position and Velocity Error for $\Delta \hat{\omega}_{\theta}(0)=0.001, \Delta \hat{\omega}_{\phi}(0)=$

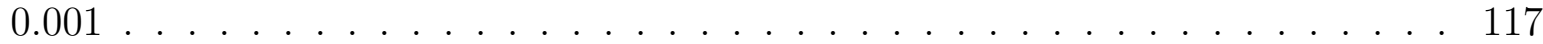




\section{Chapter 1}

\section{Introduction}

\section{$1.1 \quad$ Problem Statement}

The traditional development of relative motion equations results from a linearized model of orbital mechanics. It is the purpose of this thesis to develop relative motion equations that result from nonlinear theory. Relative motion equations are used very often in the field of orbital mechanics because an explicit dependence on time can be obtained, whereas the exact theory contains a transcendental relationship between the position and velocity of the satellite to time. There are other ways to obtain approximations that lead to this explicit time dependence, but a relative motion approach is arguably the most conceptually straightforward of these.

Figure (1.1) shows the geometry of the relative motion problem. The goal is to find the position vector, $\mathbf{R}$, from a reference point on a known orbit, $\mathbf{r}_{0}$, to a satellite, $\mathbf{r}$, as an explicit function of time. The relative velocity, $\mathbf{V}$, must also be known in order to completely solve the problem. Note that it is possible for the reference point to be occupied by another satellite.

Assuming the magnitude of $\mathbf{R}$ and $\mathbf{V}$ to be small compared with that of position and velocity of the reference orbit allows for the governing equations of motion to be approximated by using a truncated series expansion. Often this series is truncated after only the first term, resulting in a linear expression of the dynamics. There have been several attempts at developing relative motion equations that take into account nonlinear dynamics. While these solutions are much more accurate than the solutions resulting from the linearized model, they are limited to only small time periods where the solution will be valid. This thesis will develop nonlinear relative motion equations that do not have these problems.

The nonlinear relative motion equations will be found by applying a standard analytical technique used for examining nonlinear ordinary differential equations and determining approximate solutions of these equations. This technique can be applied to any system of equations that contain a small nonlinearity, and it seeks to construct a series solution that builds upon the solution of the linear form of the governing equations. The method is based on the method

of variation of parameters, where constants of integration that result from the linear solution 


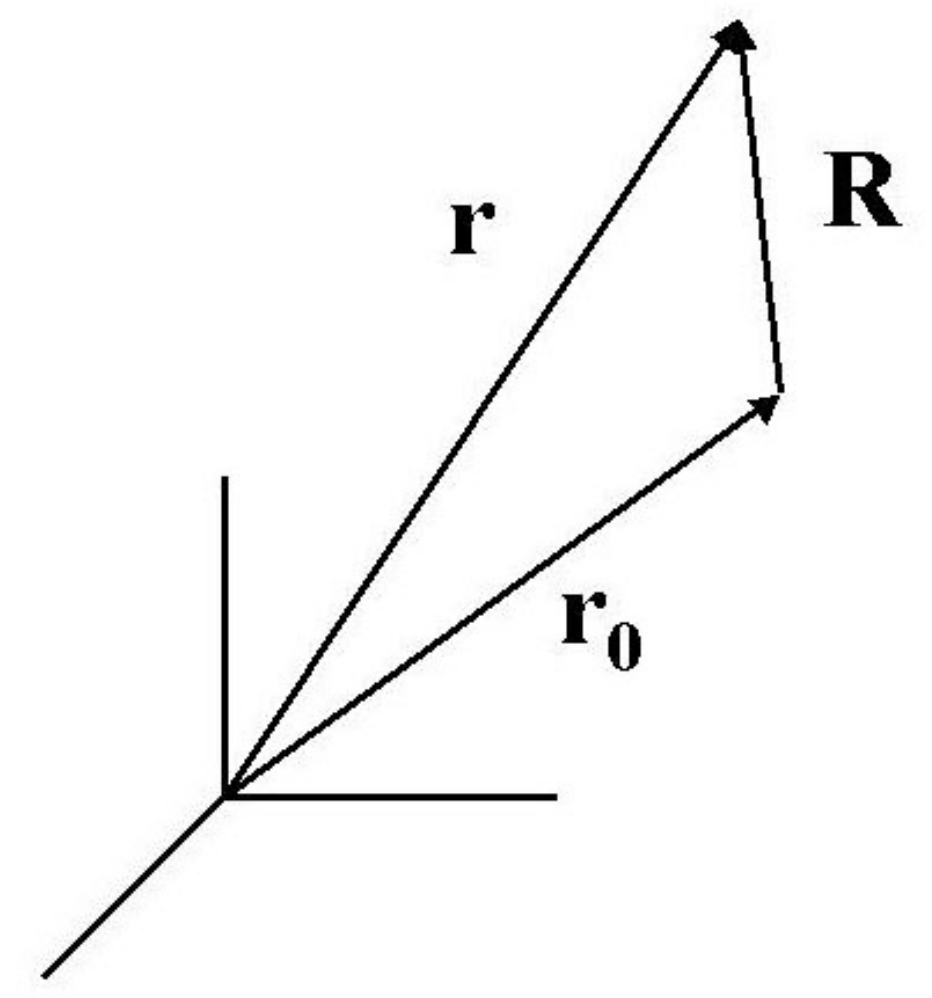

Figure 1.1: Geometry of the Relative Motion Problem

are instead allowed to be functions of time.

The accuracy of the new nonlinear relative motion equations is evaluated and compared against the linear solution using numerical integration of the full nonlinear equations of motion as truth. Comparison of the new equations is also made with numerical integration of the nonlinear equations that formed the basis for the new result, in order to understand the error induced by applying the approximation techniques.

\subsection{Applications}

Relative motion equations have seen several different application areas in the history of orbital mechanics. The first use was by Hill [1] in the late $19^{\text {th }}$ century who was studying the motion of the Moon. His goal was to construct a more mathematically sound means of developing tables of lunar motion, which, at the time, were based on "practical astronomy rather than of mathematics" in his words.

The first aerospace applications were in the area of intercept and rendezvous mechanics during the late 1950's and continuing today. The intercept problem is one in which a chase vehicle is forced in such a way that its path intersects the path of a target point (which may be occupied by another vehicle) at a specified time. The rendezvous problem further insists that 
the relative velocity of the two spacecraft be driven to zero at the time of intersection so that a docking procedure or other such activities may be conducted. This problem was studied by Clohessy and Wiltshire [2] in the interest of developing a guidance scheme for the rendezvous problem assuming that the target vehicle was in a circular orbit. This target satellite was to be a control center issuing relative position and velocity data to the slave satellites, which then used an on-board propulsion system to carry out the rendezvous and docking maneuver.

Anthony and Sasaki [3] further studied the rendezvous problem after developing a higher order approximation of the relative motion equations. Using these new equations, the velocity impulse requirements for the rendezvous maneuver were developed and an analysis of the miss distance due to the approximation was conducted.

Werlwas [4], using a new set of relative motion equations, developed an optimal two impulse approach to the intercept and rendezvous problem. This approach minimized the fuel consumed in the process. A comparison was also made with the solution resulting from the new relative motion equations and those of reference [2]

Jezewski and Donaldson [5] also considered a fuel-optimal approach to the rendezvous problem using the equations of reference [2]. A transformation of variables is introduced into the problem which leads to a closed form expression for the optimal thrust times. The problem is also constrained in such a way that the exhaust plume of the spacecraft's thrusters do not interfere with its payload.

Kelly [6] developed an optimal solution to the two impulse rendezvous problem using relative motion equations and also includes the effects of eccentric orbits and gravity perturbations. A nonlinear model of relative motion was also given, but an analytical solution was not developed and so required numerical integration to solve the problem.

Lutze [7] used relative motion equations to generate intercept and rendezvous charts for use by untethered space shuttle astronauts with maneuvering units, so that this astronaut can determine the thrust magnitude and direction in order to return to the shuttle. Furthermore, charts are also developed which instruct the astronaut on how to null the relative velocity at the time of intercept in order to rendezvous safely. Analyses are also presented to quantify errors due to the possibility of either incorrect relative position and velocity determination or improper application of the thrust.

More recent applications of relative motion concepts are in the area of satellite formations. Satellite formations are of great interest, because it is thought that large numbers of simple, low efficiency satellites working in a cohesive fashion can produce better results than a single, high performance satellite. Such formations can also achieve a greater cost effectiveness, chance of mission success and flexibility. Satellite formations are useful for Earth observing missions, where distributed groups of low resolution instruments, operating in conjunction with one another, can provide a higher overall data quality than a single, high resolution instrument.

Relative motion equations have been useful in the design and optimization of satellite formation geometry, as well as for developing control algorithms to maintain these formations. Sabol et al [8], [9] determined the orbit parameters of a formation type given the desired motion of 
that formation as determined from relative motion equations. In-plane, in-track, circular and projected circular formation types are analyzed. Furthermore, these formations are propagated forward in time in order to understand how the formation evolves as well as to estimate the fuel usage required to maintain the formation. The circular and projected circular formations were found to be extremely costly in this regard.

Chao et al [10] considered a similar problem, but developed formation geometry based on relative orbit parameters and then uses the linearized relative motion equations to visualize and further examine the relative orbits of the formation. A perturbation study was also conducted to examine the long term behavior of the formation. Based on these results, a formation keeping strategy developed from the linearized dynamics was also suggested.

Hughes [11] and Hughes and Hall [12] developed performance measures for satellite formations. Diamond formations and rotating formations (variations of the circular and projected circular formations of [8]) are considered. These performance measures are evaluated using a numerical integration scheme, but approximate analytical results are also obtained using the linearized relative motion equations. A good agreement was found between the two methods.

Badesha et al [13] used the equations of [2] modified to include atmospheric drag forces to investigate the deployment and initialization of a cluster of six satellites in an in-track formation. The investigation focused on determining possible collision scenarios between the satellites during the deployment phase, and the effects of error in the deployment velocity as well as error in the time spacing between subsequent deployments. No study was conducted to determine the effect of error in the deployment direction. A procedure was outlined for determining the amount of fuel needed to initialize the formation.

Chichka [14] used a relative motion solution to characterize the relative orbits of satellite formations that appear to have a constant distribution from an observer located on the planetary surface. A more detailed study was then conducted in order to ascertain the error made in the linearized study.

Another technique for controlling satellite formations, based on relative motion equations, is found in [15]. This technique used the relative motion equations to design periodic relative orbits of a deputy with respect to a chief. A control was then developed in order to cancel a simple gravity perturbation model in such a way that the average fuel consumption of each satellite is minimized.

\subsection{Previous Work}

Hill [1] developed a set of relative motion equations in the context of the 3-body problem in order to develop a mathematical basis for the motion of the moon in the Sun-Earth-moon system. A rotating rectangular coordinate system was used to develop these equations by using Lagrange's equations and Jacobi's integral. It was assumed that each body had a perfect inverse square gravitational field and that the mass of the moon was small compared with that of the Earth 
and Sun. Hill did not linearize the equations of motion, but rather studied the properties of the nonlinear equations. A solution of the motion was given in terms of a power series in time. The solution was not completely analytical because interpolation and numerical integration was required to determine the coefficients of the power series. This solution diverges and therefore can only be valid for short time intervals. Solutions that exhibit this type of behavior are said to contain secular terms. A definition of secular terms will be given later, but for now it suffices to say that these terms exhibit unbounded growth in time. This unbounded growth of position and velocity of the satellite does not satisfy certain principles such as conservation of momentum and energy. The accuracy of this solution is also limited by the number of terms kept in the series. Hill's solution to this problem only includes motion within the plane of the Sun-Earth-moon system.

Clohessy and Wiltshire [2] took the differential equations examined in [1] and further assumed that the satellite was moving under the influence of a linear gravity field of just one body. This linearization produced a system of constant coefficient ordinary differential equations that allowed for a simple closed form solution. The origin of the coordinate system used was a point in a circular orbit around the body. The equations that describe the motion of the satellite in the same plane as the reference circular orbit were found to de-couple from the motion perpendicular to this plane under the assumption of a linear gravity field. De-coupling is an advantage in the sense that it led to a simple solution, but is truly a disadvantage because a coupling of motion does in fact exist.

London [16] carried the work of [2] to the next level and assumed that the satellite was influenced by a quadratic gravity field. The same coordinate system of [2] was used. The assumption of a quadratic gravity field led to a re-coupling of motion in the plane and out of the plane of the reference orbit. A straightforward expansion (defined in detail in chapter (2)) was used to obtain an approximate solution of the nonlinear differential equations, as an exact solution cannot be found. This method is referred to as the method of successive approximations in many sources. The solution of the linear problem determined in [2] was used as the foundation for this approximation. The resulting solution was found to be very accurate compared with the linear solution over a period of two revolutions of the reference orbit. The solution does contain secular terms which limits the time interval over which the solution is valid, much like the solution given in [1]. Another problem with this solution is that it contains a constant offset term in the equation describing the motion out of the plane of the reference orbit. It will be shown in chapter (2) that this behavior also does not satisfy the basic physics of the problem.

De Vries [17] considered the problem of relative motion in an elliptical orbit. The equations of motion are linear and expressed using the true anomaly of the reference orbit as the independent variable. A straightforward technique is used to approximate these equations to first order in eccentricity. Secular terms result as well as the constant offset in the out of plane motion. This constant offset does vanish for the case of a circular orbit. This solution still requires a numerical procedure to evaluate the solution as a function of time, which is a disadvantage for most applications of relative motion equations. No plots are given to demonstrate the performance of the solution. 
Anthony and Sasaki [3] built upon the work of London by referencing the equations of motion to a slightly elliptical orbit rather than a circular orbit. It was assumed that the gravity field of the primary body was quadratic, and that eccentric effects beyond first order were negligible. These assumptions lead to a time varying system of nonlinear differential equations, which was also solved with a straightforward expansion. The solution is also limited in its validity range by the presence of secular terms similar to those found in previous studies. A constant offset term in the out of plane motion also appears. It was also found that there is a difference between the new solution and that developed in [16] in the case where the reference orbit is circular. The new solution was shown to improve on the equations given in [2] for two revolutions of the reference orbit.

Euler and Shulman [18] considered the motion of a satellite with respect to an arbitrary elliptical orbit keeping quadratic terms in the equations of motion. True anomaly was used as the independent variable. A straightforward expansion was employed to obtain a solution of this problem, but the result cannot be expressed analytically as numerical integration was still required. Dependence on numerical integration, combined with the fact that a numerical scheme was required to evaluate the solution as a function of time, severely limits the usefulness of the solution. The solution is not given, and hence it is not clear if secular terms or the constant offset in the out-of-plane motion appear. Several plots were given which show improvement over a linear solution, although it is not clear which linear solution this is. These plots are only given for the out-of-plane motion, and the new solution is found to be valid for approximately one half of the period of the reference orbit while the linear solution is valid for only one quarter of the period for the example given.

Lancaster [19] gave a numerical scheme for determining the relative motion of two orbits in terms of the eccentric anomaly. Although it is shown for one example that the method is very accurate, a numerical procedure looses much of the convenience that is possible from an analytical solution.

Werlwas [4] develoed a new solution of the relative motion problem by placing the origin of the reference frame on the satellite itself rather than a point on a reference orbit. The equations of motion were linearized with respect to this configuration. This formulation lead to a complicated, although accurate solution. A limitation of the solution was that there can be no initial out of plane displacement, although an initial velocity out of plane is allowable. The form of the solution also depends on the initial conditions, which is a disadvantage as well.

Berreen and Crisp [20] developed a new linear solution for the relative motion of a satellite with respect to an elliptical reference orbit using true anomaly as the independent variable. The solution requires that these two orbits be coplanar. It is true that all Keplerian motion is restricted to a single plane, so for a given set of initial position and velocity its motion can be determined relative to a any orbit in the same plane. Since the properties of the reference orbit are known, this motion can then be determined in any other coordinate system by a series of simple transformations. However, most applications of interest, such as intercept or rendezvous, will in general have two vehicles in non-coplanar orbits, so this restriction is a disadvantage when considering practical problems. The authors also pointed out that the accuracy of the 
original linear solution given in [2] can be improved by essentially transforming the solution to cylindrical coordinates.

Szebehely [21] presented three transformations between various anomalies and time that can be used to improve the accuracy of determining the relative motion of two particles when evaluated numerically. No restrictions are placed on the eccentricity of the orbits or the size of their relative displacements. Such a scheme could be employed to further improve upon the work of Lancaster or Euler and Shulman.

Kelly [6] reformulated the problem by including the effects of a non-spherical gravity perturbation. The motion of a satellite under this perturbed gravity field is referenced to a point on an arbitrary un-perturbed orbit. The equations of motion are developed in a non-orthogonal coordinate system with time-varying coefficients. A straightforward perturbation technique was used to obtain an approximate solution of this system of equations. The actual solution was not presented, although it was mentioned that it does contain secular terms. It was not mentioned if the solution contains the constant offset in the out-of-plane solution.

Garrison et al [22] gave the solution of a linear set of relative motion equations referenced to an elliptical orbit. This solution again uses true anomaly as the independent variable and therefore still requires a numerical procedure for determining the solution as a function of time. The results are plotted for several sets of initial conditions and are found to be accurate for up to two revolutions of the reference orbit, although for very large initial separations the results were be not as good. A study was also conducted to understand the error induced by the linear representation of the dynamics as well as the effects of a non-spherical central body and other perturbations that were ignored in the model. It was determined that the error made by the linearization is much greater than that of the perturbations over the course of two revolutions of the reference orbit.

The most recent work on this subject is by Melton [23], [24] who developed a linear set of relative motion equations for an elliptical orbit including second order powers of eccentricity in the approximation. The advantage of this solution over many of the previous solutions for the case of an elliptical reference orbit is that the equations are given explicitly in terms of time, hence no iterative technique is required to relate anomaly to time. The solution was given in both rectangular and cylindrical coordinates, and can include the effects of certain forms of perturbations through a convolution integral. The cylindrical coordinate representation was found to be more accurate than the rectangular coordinate solution. Plots were given for a set of initial conditions which shows the error of the new solution for each order of eccentricity, which improves as more terms are kept in the expansion. Secular terms are present which limits the time interval over which the solution will be valid.

Note several general trends in the history of this problem. It seems that any attempt at considering nonlinear terms in the equations of motion results in a constant offset in the out-ofplane motion. Also, any attempt at extending the linear equations of motion about a circular orbit to considering any combination of nonlinear or eccentric effects also results in false secular terms. No analytic solution that improves the results of [2] has been given which does not exhibit at least one these two false behaviors. 


\subsection{Overview}

The rest of this thesis continues with a review of several basic concepts from orbital mechanics and mathematics. This review is brief and only includes what is necessary to give a complete picture of this problem. This summary starts with an overview of orbital mechanics, the TwoBody problem and several of its basic solutions. Next, a review of several analytical techniques used to solve systems of nonlinear and/or nonautonomous ordinary differential equations is presented. An example of a nonlinear oscillator is given to illustrate the application these methods.

Chapter (3) focuses on the previously known linear model and its solutions, as it is necessary to have an understanding of the linear solution before attempting to find a nonlinear solution. These equations result from a Taylor expansion of the two body equations of motion. This expansion is derived, and then the solution is developed in spherical coordinates. This solution is often called Hill's equations, or the Clohessy-Wiltshire equations. The advantages and disadvantages of the linear model are also discussed.

Chapter (4) discusses the nonlinear model. The two body equations of motion are expanded in Taylor series form, very much like the linear model, although now terms that contain quadratic nonlinearities are also kept. Then the two perturbation techniques are employed to find the solution of the resulting nonlinear differential equations.

In chapter (5) a comparison is drawn between the previously known linear solution, the new approximate analytical solution found in chapter (4), and numerical integration of the quadratic equations of motion. These are all compared with numerical integration of the full nonlinear equations of motion. 


\section{Chapter 2}

\section{Preliminaries}

It is the purpose of this chapter to review several basic concepts from the areas of orbital mechanics and mathematics. This review is conducted in order to set down a notation and language style more than anything else, so detailed proofs are not given, although they are referenced.

\subsection{Orbital Mechanics}

\subsubsection{The Two-Body Problem}

The basic assumptions of the Two-Body problem are [25]:

1. The equations of motion are expressed in a non-inertial reference frame and the origin of that frame coincides with the center of mass of the central body

2. The central body and satellite are both homogeneous spheres or points of equivalent mass

3. The inverse-square gravitational force between the two bodies is the only force in action

Under these assumptions, the governing equation is

$$
\ddot{\mathbf{r}}=-\frac{\mu}{r^{3}} \mathbf{r}
$$

where $\mathbf{r}$ is the vector from the center of mass of the central body to the satellite in question and $\mu$ is the gravitational parameter. The value of $\mu$ is equal to $G(M+m)$, where $G$ is the universal gravitational constant, $M$ is the mass of the central body and $m$ is the mass of the satellite. A further assumption that is commonly made is that the mass of the satellite, $m$, is extremely small compared to the mass of the central body $M$. In the limit as $m \rightarrow 0$, the center of mass of the central body becomes an inertial reference frame. 
The determination of such an inertial reference frame relies on first choosing a fundamental plane. Traditionally, the $x$ axis of the system is chosen to be in some fundamental direction within that plane, the $y$ axis being perpendicular to the $x$ axis but also within the fundamental plane. The $z$ axis is taken to be perpendicular to the plane in a right-handed sense. For the Earth, the fundamental plane is the equatorial plane, the $x$ axis is in the direction of the vernal equinox and the $z$ axis coincides with the axis of rotation of the Earth. This frame is commonly called the Earth-Centered Inertial frame. In the case of the Sun, the fundamental plane is the plane of the Earth's orbit, the $x$ axis is also in the direction of the vernal equinox and the $z$ axis in the direction normal to the orbit plane. This frame is known as the Heliocentric Coordinate System. For both frames, the direction of the $x$ axis is found by drawing a line from the center of the Earth through the center of the Sun at the precise moment of the vernal equinox (the first day of spring). This choice of $x$ axis is traditional - there are an infinite number of other valid choices of inertial reference frames for both of these systems. Note that these frames are not truly inertial because they are in fact accelerating. It is a useful first approximation to assume that they are inertial, and corrections can be made to the results of this assumption to account for this acceleration as well as other perturbations.

Several constants of motion can be extracted from equation (2.1). First, an energy equation can be found from the dot product of (2.1) with the velocity vector $\mathbf{v}$

$$
\begin{aligned}
\mathbf{v} \cdot \dot{\mathbf{v}} & =-\frac{\mu}{r^{3}} \mathbf{v} \cdot \mathbf{r} \\
v \dot{v} & =-\frac{\mu}{r^{3}} r \dot{r}
\end{aligned}
$$

Note that

$$
\frac{d}{d t}\left(\frac{v^{2}}{2}-\frac{\mu}{r}\right)=0
$$

or, that

$$
\frac{v^{2}}{2}-\frac{\mu}{r}=\text { constant }
$$

An angular momentum equation can be found from the cross product of (2.1) with the radius vector, $\mathbf{r}$,

$$
\mathbf{r} \times \ddot{\mathbf{r}}=-\frac{\mu}{r^{3}} \mathbf{r} \times \mathbf{r}=0
$$

Note that

$$
\frac{d}{d t}(\mathbf{r} \times \mathbf{v})=\mathbf{v} \times \mathbf{v}+\mathbf{r} \times \dot{\mathbf{v}}
$$

therefore, it must be true that

$$
\mathbf{r} \times \mathbf{v}=\text { constant }
$$

Equation (2.7) states that an orbit must always be restricted to a plane, and that this plane must always contain the origin of the inertial coordinate system located at the center of mass of the system. Figure (2.1) shows a path that contains a constant offset out of the plane of a reference orbit as in the solution given in reference [16]. This constant offset defies the conservation of angular momentum, since the plane of this orbit does not pass through the origin of the coordinate system. 


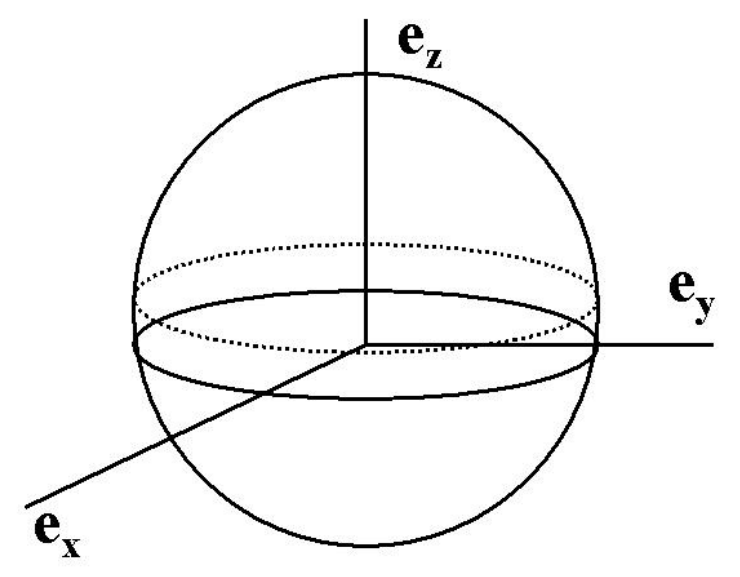

Figure 2.1: Orbit with Constant Out-of-Plane Offset

\subsubsection{Equations of Motion}

The coordinate systems shown in figure (2.2) will be used to further develop equation (2.1). The coordinate system described by $\mathbf{e}_{x}, \mathbf{e}_{y}$ and $\mathbf{e}_{z}$ are the unit vectors of an inertial coordinate system (which may be any of those discussed previously) that uses the variable $x, y$ and $z$ to locate a point in three dimensions. The coordinate system $\mathbf{e}_{r}, \mathbf{e}_{\theta}$ and $\mathbf{e}_{\phi}$ are the unit vectors of a spherical coordinate system and uses variable $r, \theta$ and $\phi$. In inertial coordinates, the vector from the origin to a point of interest is

$$
\mathbf{r}=x \mathbf{e}_{x}+y \mathbf{e}_{y}+z \mathbf{e}_{z}
$$

The same vector in spherical coordinates is

$$
\mathbf{r}=r \mathbf{e}_{r}
$$

The spherical coordinate system may be related to the inertial coordinate system by a series of two simple coordinate transformations. First, the inertial frame is rotated by an angle $\theta$ about the $\mathbf{e}_{z}$ axis, to an intermediate axis system $\mathbf{e}_{\tilde{x}}, \mathbf{e}_{\tilde{y}}$ and $\mathbf{e}_{\tilde{z}}$. This intermediate system is then rotated about axis $\mathbf{e}_{\tilde{y}}$ by an angle $-\phi$. This operation may be expressed in matrix form 


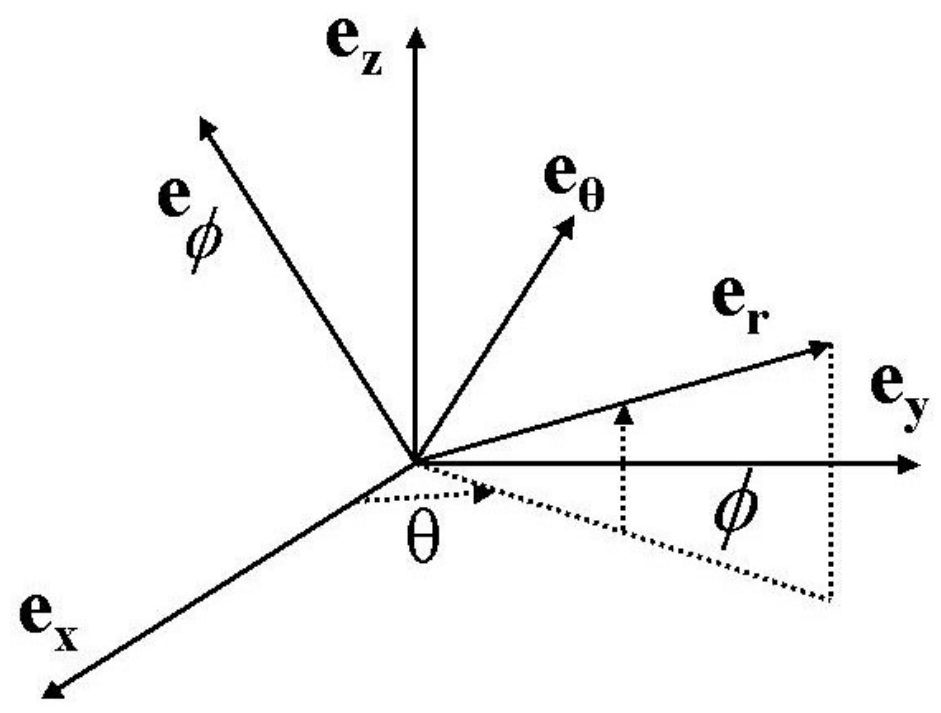

Figure 2.2: Spherical Coordinate System

as:

$$
\begin{aligned}
\left(\begin{array}{l}
\mathbf{e}_{r} \\
\mathbf{e}_{\theta} \\
\mathbf{e}_{\phi}
\end{array}\right)= & {\left[\begin{array}{ccc}
\cos \phi & 0 & \sin \phi \\
0 & 1 & 0 \\
-\sin \phi & 0 & \cos \phi
\end{array}\right]\left[\begin{array}{ccc}
\cos \theta & \sin \theta & 0 \\
-\sin \theta & \cos \theta & 0 \\
0 & 0 & 1
\end{array}\right]\left(\begin{array}{l}
\mathbf{e}_{x} \\
\mathbf{e}_{y} \\
\mathbf{e}_{z}
\end{array}\right) } \\
= & {\left[\begin{array}{ccc}
\cos \phi \cos \theta & \cos \phi \sin \theta & \sin \phi \\
-\sin \theta & \cos \theta & 0 \\
-\sin \phi \cos \theta & -\sin \theta \sin \theta & \cos \phi
\end{array}\right]\left(\begin{array}{l}
\mathbf{e}_{x} \\
\mathbf{e}_{y} \\
\mathbf{e}_{z}
\end{array}\right) }
\end{aligned}
$$

Since the direction of the inertial unit vectors is fixed, the derivatives of the spherical unit vectors are simply

$$
\begin{aligned}
\left(\begin{array}{c}
\dot{\mathbf{e}}_{r} \\
\dot{\mathbf{e}}_{\theta} \\
\dot{\mathbf{e}}_{\phi}
\end{array}\right) & =\left[\begin{array}{ccc}
-\dot{\phi} \sin \phi \cos \theta-\dot{\theta} \cos \phi \sin \theta & -\dot{\phi} \sin \phi \sin \theta+\dot{\theta} \cos \phi \cos \theta & \dot{\phi} \cos \phi \\
-\dot{\theta} \cos \theta & -\dot{\theta} \sin \theta & 0 \\
-\dot{\phi} \cos \phi \cos \theta+\dot{\theta} \sin \phi \sin \theta & -\dot{\phi} \cos \phi \sin \theta-\dot{\theta} \sin \phi \cos \theta-\dot{\phi} \sin \phi
\end{array}\right]\left(\begin{array}{l}
\mathbf{e}_{x} \\
\mathbf{e}_{y} \\
\mathbf{e}_{z}
\end{array}\right) \\
& =\left[\begin{array}{ccc}
0 & \dot{\theta} \cos \phi & \dot{\phi} \\
-\dot{\theta} \cos \phi & 0 & \dot{\theta} \sin \phi \\
-\dot{\phi} & -\dot{\theta} \sin \phi & 0
\end{array}\right]\left(\begin{array}{l}
\mathbf{e}_{r} \\
\mathbf{e}_{\theta} \\
\mathbf{e}_{\phi}
\end{array}\right)
\end{aligned}
$$

Therefore, the velocity of the point of interest is

$$
\begin{aligned}
\dot{\mathbf{r}} & =\dot{r} \mathbf{e}_{r}+r \dot{\mathbf{e}}_{r} \\
& =\dot{r} \mathbf{e}_{r}+r \dot{\theta} \cos \phi \mathbf{e}_{\theta}+\dot{\phi} \mathbf{e}_{\phi}
\end{aligned}
$$


and the acceleration is

$$
\begin{aligned}
\ddot{\mathbf{r}}= & \ddot{r} \mathbf{e}_{r}+\dot{r} \dot{\mathbf{e}}_{r}+(\dot{r} \dot{\theta} \cos \phi+r \ddot{\theta} \cos \phi-r \dot{\theta} \dot{\phi} \sin \phi) \mathbf{e}_{\theta}+r \dot{\theta} \cos \phi \dot{\mathbf{e}}_{\theta}+(\dot{r} \dot{\theta}+r \ddot{\phi}) \mathbf{e}_{\phi}+r \dot{\phi} \dot{\mathbf{e}}_{\phi} \\
= & \left(\ddot{r}-r \dot{\theta}^{2} \cos ^{2} \phi-r \dot{\phi}^{2}\right) \mathbf{e}_{r}+(r \ddot{\theta} \cos \phi+2 \dot{r} \dot{\theta} \cos \phi-2 r \dot{\theta} \dot{\phi} \sin \phi) \mathbf{e}_{\theta}+ \\
& +\left(r \ddot{\phi}+2 \dot{r} \dot{\phi}+r \dot{\theta}^{2} \sin \phi \cos \phi\right) \mathbf{e}_{\phi}
\end{aligned}
$$

Relating (2.13 to the expression given in (2.1), the equations of motion for a particle in spherical coordinates are

$$
\begin{aligned}
\ddot{r}-r \dot{\theta}^{2} \cos ^{2} \phi-r \dot{\phi}^{2}+\frac{\mu}{r^{2}} & =0 \\
r \ddot{\theta} \cos \phi+2 \dot{r} \dot{\theta} \cos \phi-2 r \dot{\theta} \dot{\phi} \sin \phi & =0 \\
r \ddot{\phi}+2 \dot{r} \dot{\phi}+r \dot{\theta}^{2} \sin \phi \cos \phi & =0
\end{aligned}
$$

Note that $(2.14-2.16)$ is a sixth order system of coupled, nonlinear ordinary differential equations. An exact solution of these equations is not possible, hence the need to introduce techniques that can be used to find approximate solutions.

\subsection{Perturbation Methods}

This section discusses several techniques that may be employed to find an approximate analytical solution of a system of differential equations. Typically these methods are applied to problems where the exact solution is either impossible or impractical to find by other means. Such systems are often nonlinear and/or nonautonomous in nature. Approximation techniques exist for both ordinary and partial differential equations, but for the purposes here only a discussion of the techniques for nonlinear ordinary differential equations is needed. For a more detailed description of these types of problems, see reference [26].

The analytical techniques discussed in this section require that the system be weakly nonlinear or weakly nonautonomous, meaning that those terms in the equation containing the nonlinearity or nonautonomy are small. Alternatively, systems of this nature can be thought of as almost linear, or quasi-linear. A consequence of almost linear systems is that the differential equations will have linear terms and small nonlinear or nonautonomous terms separated from each other. The disturbing terms are commonly known as perturbations, and the class of analytical techniques used to treat these systems are known as perturbation methods. These perturbation terms are typically represented by a coefficient whose value is very small, and the perturbation methods discussed here construct a power series solution in this small parameter.

Unfortunately, it is also quite common to encounter problems that do not contain a small parameter. In this event, an approximation may still be found by introducing a parameter into the problem to be used if it were small, but in the end its value set to 1 . This technique is valid when the terms rendering the differential equation quasi-linear are small in and of themselves. Parameters introduced in this way are often called place keeping parameters. 
Next an overview of three perturbation methods is given. The discussion of each method is motivated by a weakly nonlinear mass-spring system given by

$$
\ddot{x}+x=\epsilon f(x, \dot{x})
$$

where $x$ is a non-dimensional displacement, $f(x, \dot{x})$ is a disturbing function and $\epsilon$ is very small compared with 1 . It is also possible that $\epsilon$ is a place keeping parameter that has been introduced into the system. Since this is a second order ordinary differential equation, two conditions must be prescribed in order to solve for the constants of integration which will appear in the solution process. For this discussion a set of initial conditions is assumed, where

$$
\begin{aligned}
& x(0)=a \\
& \dot{x}(0)=b
\end{aligned}
$$

\subsubsection{The Straightforward Expansion}

The most straightforward technique for finding analytical solutions of almost linear systems is, naturally, called the straightforward technique. This method seeks a power series solution of the form

$$
x(t, \epsilon)=x_{0}(t)+\epsilon x_{1}(t)+\epsilon^{2} x_{2}(t)+\cdots
$$

where the $\epsilon x_{1}, \epsilon^{2} x_{2}$ and so on are small correction terms to $x_{0}$. The number of such correction terms needed depends on the size of $\epsilon$ and the required accuracy of the approximation.

Substituting (2.20) into (2.17) and keeping only terms of order $\epsilon$ leads to

$$
\ddot{x_{0}}+\epsilon \ddot{x_{1}}+x_{0}+\epsilon x_{1}=f\left(x_{0}+\epsilon x_{1}, \dot{x_{0}}+\epsilon \dot{x_{1}}\right)+\cdots
$$

The function $f$ may be expanded in a Taylor series about the point $\epsilon=0$, leading to

$$
f\left(x_{0}+\epsilon x_{1}, \dot{x_{0}}+\epsilon \dot{x_{1}}\right)=f\left(x_{0}, \dot{x_{0}}\right)+\epsilon x_{1} \frac{\partial f}{\partial x}\left(x_{0}, \dot{x_{0}}\right)+\epsilon \dot{x_{1}} \frac{\partial f}{\partial \dot{x}}\left(x_{0}, \dot{x_{0}}\right)+\cdots
$$

Since this expansion must hold for any value of $\epsilon$, it is necessary to equate the coefficients of the successive powers of $\epsilon$, which leads to the equations

$$
\begin{aligned}
& \ddot{x_{0}}+x_{0}=0 \\
& \ddot{x_{1}}+x_{1}=f\left(x_{0}, \dot{x_{0}}\right)
\end{aligned}
$$

Note that (2.23) is simply the unperturbed linear system, and that (2.24) is the same system but with an input that depends on the solution of the unperturbed problem. Each of 
the differential equations may be solved independently in order to construct a series solution of the original problem.

In this procedure, a single second order ordinary differential equation has been replaced with a series of two second order ordinary differential equations. Each of these differential equations will need two conditions in order to completely solve the problem. However, only two such conditions are given in the statement of the problem. One way to deal with this requirement is to assume that the constants of integration that arise in (2.23) and (2.24) are unknown, then the initial conditions of the system are enforced when the full solution is assembled in (2.20) in such a way that the constants of integration that appear in the nonlinear solution remain defined in the same manner as the linear solution. Another method is to enforce the given initial conditions on the solution of equation (2.23) and assume that the initial conditions of (2.24) are zero, implying that the constants of integration depend on $\epsilon$. Each of these two procedures for dealing with initial conditions produce the same result. For the purposes of this paper, the first of these methods will be used.

As an example of the straightforward expansion, take again the function $f(x, \dot{x})=-x^{3}$. Equations (2.23) and (2.24) become

$$
\begin{aligned}
& \ddot{x_{0}}+x_{0}=0 \\
& \ddot{x_{1}}+x_{1}=-x_{0}^{3}
\end{aligned}
$$

The solution of (2.25) with initial conditions $x(0)=a$ and $\dot{x}(0)=b$ may be written as

$$
x_{0}=c \cos (t+\phi)
$$

where

$$
\begin{aligned}
& x(0)=x_{0}(0)+\epsilon x_{1}(0) \\
& \dot{x}(0)=\dot{x}_{0}(0)+\epsilon \dot{x}_{1}(0)
\end{aligned}
$$

which means that in order for the constants of integration to remain defined in the same manner as the linear solution, it must be true that $x_{1}(0)=0$ and $\dot{x}_{1}(0)=0$. Equation $(2.26)$ becomes

$$
\ddot{x_{1}}+x_{1}=-\frac{c^{3}}{4}[\cos (3 t+3 \phi)+3 \cos (t+\phi)]
$$

which has the general solution

$$
x_{1}=A \cos t+B \sin t+c^{3}\left[\frac{1}{32} \cos (3 t+3 \phi)-\frac{3}{8} t \sin (t+\phi)\right]
$$

Evaluating this solution with the initial conditions gives

$$
\begin{aligned}
& A=-\frac{c^{3}}{32} \cos 3 \phi \\
& B=\frac{c^{3}}{32} \sin 3 \phi+\frac{3 c^{3}}{8} \sin \phi
\end{aligned}
$$


Assembling the full solution in (2.20) results in

$$
\begin{aligned}
x(t)= & c \cos (t+\phi)+\epsilon c^{3}\left[\left(\frac{1}{32} \sin 3 \phi+\frac{3}{8} \sin \phi\right) \sin t-\frac{1}{32} \cos 3 \phi \cos t+\right. \\
& \left.+\frac{1}{32} \cos (3 t+3 \phi)-\frac{3}{8} t \sin (t+\phi)\right]
\end{aligned}
$$

where

$$
\begin{aligned}
& a=c \cos \phi \\
& b=-c \sin \phi
\end{aligned}
$$

Notice the appearance of the terms $t \sin t$. This term possess an unbounded amplitude growth over time, and at time $t=\frac{1}{\epsilon}$, the relative magnitude of the correction term $\epsilon x_{1}$ becomes the same as $x_{0}$. As time increases further, these terms begin to dominate the behavior of the solution. This unbounded growth in time violates the assumption that the correction terms are small compared with $x_{0}$. Also, this example did not contain any damping terms, meaning that the total energy is conserved. Unbounded behavior also does not satisfy these conditions. Terms that contain an unbounded growth in time are called secular terms. If it is known from either numerical integration or some form of physical insight that the solution should not exhibit this unbounded growth in time, then these secular terms are called false or spurious secular terms. A solution that contains such secular terms is often called nonuniform.

Several methods exist for dealing with these secular terms. These methods, in one form or another, all take a variation of parameters approach to the problem, and allow the constants of integration to now be unknown functions of time which are determined in the solution process. In the perturbation method case, these functions are assumed to be slowly varying.

\subsubsection{The Method of Multiple Scales}

The method of multiple scales, described in [27], [28] and [26], seeks a solution whose behavior depends on several time scales, each an order of magnitude in $\epsilon$ slower than the previous. The method of multiple scales is a generalized form of the Lindstedt-Poincaré technique (often called the method of strained coordinates) which is based on the observation that the frequency of a nonlinear oscillation may depend upon its amplitude. The frequency is expanded in an asymptotic series, and the coefficients of each term in the series are determined in such a way the the solution is free of secular terms. In a similar fashion, the method of multiple scales allows the solution vary on fast and slow time scales. The fast time scale corresponds with the first (linear) order Lindstedt-Poincaré expansion, and each slow scale matches with the second and higher terms in the expansion. The main difference is that multiple scales assumes that the coefficients of each scale are fixed (typically equal to 1 ), and uses a variation-of-parameters approach and lets the constants of integration that appear in the linear solution be functions that vary on the slow time scale. This procedure results in systems of partial differential equations at 
each order of $\epsilon$ that must be solved to produce a uniform solution of the problem. The method of multiple scales is a much more powerful tool than the Lindstedt-Poincaré technique because the former allows for all of the "constants" to vary on the slow time scales (so, for a second order differential equation there are two such parameters) while the latter only introduces one free parameter at each order of $\epsilon$.

For this problem, $t$ is the fast scale, $\epsilon t$ a slow scale, $\epsilon^{2} t$ an ever slower scale and so on. These time scales are written as $T_{0}=t, T_{1}=\epsilon t, T_{2}=\epsilon^{2} t$, and in general, $T_{k}=\epsilon^{k} t$. The dependent variable $x$ is then written in the series expansion

$$
x(t)=x_{0}\left(T_{0}, T_{1}, T_{2}, \ldots\right)+\epsilon x_{1}\left(T_{0}, T_{1}, T_{2}, \ldots\right)+\epsilon^{2} x_{2}\left(T_{0}, T_{1}, T_{2}, \ldots\right)+\cdots
$$

If a solution accuracy of order $\epsilon^{n}$ is needed, then it is necessary to keep the time scale $T_{n}$ and the correction term $\epsilon^{n} x_{n}$ in the procedure. For the case where only terms of order $\epsilon$ are required,

$$
\begin{aligned}
\dot{x} & =\frac{\partial x_{0}}{\partial T_{0}} \dot{T}_{0}+\frac{\partial x_{0}}{\partial T_{1}} \dot{T}_{1}+\epsilon \frac{\partial x_{1}}{\partial T_{0}} \dot{T}_{0}+\epsilon \frac{\partial x_{1}}{\partial T_{1}} \dot{T}_{1}+\cdots \\
& =\frac{\partial x_{0}}{\partial T_{0}}+\epsilon\left(\frac{\partial x_{0}}{\partial T_{1}}+\frac{\partial x_{1}}{\partial T_{0}}\right)+\cdots \\
\ddot{x} & =\frac{\partial^{2} x_{0}}{\partial T_{0}^{2}} \dot{T}_{0}+\frac{\partial^{2} x_{0}}{\partial T_{1} \partial T_{0}} \dot{T}_{1}+\epsilon\left(\frac{\partial^{2} x_{0}}{\partial T_{0} \partial T_{1}} \dot{T}_{0}+\frac{\partial^{2} x_{0}}{\partial T_{1}^{2}} \dot{T}_{1}+\frac{\partial^{2} x_{1}}{\partial T_{0}^{2}} \dot{T}_{0}+\frac{\partial^{2} x_{1}}{\partial T_{1} \partial T_{0}} \dot{T}_{1}\right) \\
& =\frac{\partial^{2} x_{0}}{\partial T_{0}^{2}}+\epsilon\left(2 \frac{\partial^{2} x_{0}}{\partial T_{1} \partial T_{0}}+\frac{\partial^{2} x_{1}}{\partial T_{0}^{2}}\right)
\end{aligned}
$$

Substituting equations (2.39) and (2.37) into (2.17) and grouping powers of $\epsilon$ yields

$$
\begin{aligned}
& \frac{\partial^{2} x_{0}}{\partial T_{0}^{2}}+x_{0}=0 \\
& \frac{\partial^{2} x_{1}}{\partial T_{0}^{2}}+x_{1}=f\left(x_{0}, \dot{x_{0}}\right)-2 \frac{\partial^{2} x_{0}}{\partial T_{1} \partial T_{0}}
\end{aligned}
$$

Equation (2.40) is similar to a mass-spring system, although since it is now a partial differential equation the constants of integration are instead functions of the other variables, in this case $T_{1}$. Therefore, the solution of this equation is

$$
x_{0}=c\left(T_{1}\right) \cos \left[T_{0}+\phi\left(T_{1}\right)\right]
$$

which can also be written as

$$
x_{0}=A\left(T_{1}\right) \mathrm{e}^{i T_{0}}+\bar{A}\left(T_{1}\right) \mathrm{e}^{-i T_{0}}
$$

where $A=\frac{1}{2} c e^{i \phi}$, and $\bar{A}$ is its complex conjugate. The method of multiple scales lends itself well to using the latter form of the solution. The initial conditions of the problem (to order $\epsilon$ ) are

$$
\begin{aligned}
& x(0)=x_{0}(0)+\epsilon x_{1}(0) \\
& \dot{x}(0)=\frac{\partial x_{0}}{\partial T_{0}}(0)+\epsilon\left(\frac{\partial x_{0}}{\partial T_{1}}(0)+\frac{\partial x_{1}}{\partial T_{0}}(0)\right)
\end{aligned}
$$


and therefore,

$$
\begin{aligned}
x_{1}(0) & =0 \\
\frac{\partial x_{1}}{\partial T_{0}}(0) & =-\frac{\partial x_{0}}{\partial T_{1}}(0)
\end{aligned}
$$

Equation (2.41) with $f(x, \dot{x})=-x^{3}$ becomes

$$
\begin{aligned}
\frac{\partial^{2} x_{1}}{\partial T_{0}^{2}}+x_{1}= & -2 i \frac{\partial A}{\partial T_{1}} \mathrm{e}^{i T_{0}}+2 i \frac{\partial \bar{A}}{\partial T_{1}} \mathrm{e}^{-i T_{0}}-\left(A\left(T_{1}\right) \mathrm{e}^{i T_{0}}+\bar{A}\left(T_{1}\right) \mathrm{e}^{-i T_{0}}\right)^{3} \\
= & -\left(2 i \frac{\partial A}{\partial T_{1}}+3 A^{2} \bar{A}\right) \mathrm{e}^{i T_{0}}+\left(2 i \frac{\partial \bar{A}}{\partial T_{1}}-3 \bar{A}^{2} A\right) \mathrm{e}^{-i T_{0}}- \\
& -A^{3} \mathrm{e}^{3 i T_{0}}-\bar{A}^{3} \mathrm{e}^{-3 i T_{0}}
\end{aligned}
$$

which has the general solution

$$
\begin{aligned}
x_{1}= & \frac{1}{2} i\left(2 i \frac{\partial A}{\partial T_{1}}+3 A^{2} \bar{A}\right) T_{0} \mathrm{e}^{i T_{0}}+\frac{1}{2} i\left(2 i \frac{\partial A}{\partial T_{1}}-3 \bar{A}^{2} A\right) T_{0} \mathrm{e}^{-i T_{0}}+ \\
& +\frac{A^{3}}{8} \mathrm{e}^{3 i T_{0}}+\frac{\bar{A}^{3}}{8} \mathrm{e}^{-3 i T_{0}}+B \mathrm{e}^{i T_{0}}+\bar{B} \mathrm{e}^{-i T_{0}}
\end{aligned}
$$

In order to eliminate secular terms, it must be true that

$$
\begin{aligned}
& 2 i \frac{\partial A}{\partial T_{1}}+3 A^{2} \bar{A}=0 \\
& 2 i \frac{\partial \bar{A}}{\partial T_{1}}-3 \bar{A}^{2} A=0
\end{aligned}
$$

Note that (2.51) is simply the complex conjugate of (2.50), so no new information is contained within it. Making the transformation

$$
A=\frac{1}{2} c \mathrm{e}^{i \phi}
$$

results in

$$
\frac{\partial A}{\partial T_{1}}=\frac{1}{2} \frac{\partial c}{\partial T_{1}} \mathrm{e}^{i \phi}+\frac{1}{2} i c \frac{\partial \phi}{\partial T_{1}} \mathrm{e}^{i \phi}
$$

Substituting this into (2.50) and multiplying through by $\mathrm{e}^{-i \phi}$ yields

$$
i \frac{\partial c}{\partial T_{1}}-c \frac{\partial \phi}{\partial T_{1}}+\frac{3 c^{3}}{8}=0
$$

Equating real and imaginary parts,

$$
\begin{gathered}
\frac{\partial c}{\partial T_{1}}=0 \\
c \frac{\partial \phi}{\partial T_{1}}=\frac{3 c^{3}}{8}
\end{gathered}
$$


which leads to

$$
\begin{aligned}
c & =c_{0} \\
\phi & =\frac{3 c_{0}^{3}}{8} T_{1}+\phi_{0}
\end{aligned}
$$

Evaluating the initial conditions gives

$$
\begin{aligned}
B+\bar{B}= & -\frac{1}{8} A(0)^{3}-\frac{1}{8} \bar{A}(0)^{3} \\
B-\bar{B}= & i \frac{\partial A}{\partial T_{1}}(0)+i \frac{\partial \bar{A}}{\partial T_{1}}(0)-\frac{3}{8} A(0)^{3}+\frac{3}{8} \bar{A}(0)^{3} \\
& =-\frac{3}{2} A(0)^{2} \bar{A}+\frac{3}{2} \bar{A}(0)^{2} A(0)-\frac{3}{8} A(0)^{3}+\frac{3}{8} \bar{A}(0)^{3}
\end{aligned}
$$

Making the transformation

$$
B=\frac{1}{2} \mathrm{e}^{i \beta}
$$

and the substitution

$$
A(0)=\frac{1}{2} c_{0} \mathrm{e}^{i \phi_{0}}
$$

leads to

$$
\begin{aligned}
b \cos \beta & =-\frac{1}{32} c_{0}^{3} \cos 3 \phi_{0} \\
b \sin \beta & =-\frac{3}{8} c_{0}^{3} \sin \phi_{0}-\frac{3}{32} c_{0}^{3} \sin 3 \phi_{0}
\end{aligned}
$$

Combining these results together with (2.49), (2.43) and (2.37) gives

$$
\begin{aligned}
x & =A \mathrm{e}^{i T_{0}}+\bar{A} \mathrm{e}^{-i T_{0}}+\epsilon\left[B \mathrm{e}^{i T_{0}}+\bar{B} \mathrm{e}^{-i T_{0}}+\frac{A^{3}}{8} \mathrm{e}^{3 i T_{0}}+\frac{\bar{A}^{3}}{8} \mathrm{e}^{-3 i T_{0}}\right] \\
& =c \cos \left(T_{0}+\phi\right)+\epsilon\left[\frac{c^{3}}{32} \cos \left(3 T_{0}+3 \phi_{0}\right)+b \cos \beta \cos T_{0}-b \sin \beta \sin T_{0}\right]
\end{aligned}
$$

And therefore,

$$
\begin{aligned}
x= & c_{0} \cos \left[\left(1+\frac{3 \epsilon c_{0}^{2}}{8}\right) t+\phi_{0}\right]+\epsilon c_{0}^{3}\left[\frac{1}{32} \cos \left[3\left(1+\frac{3 \epsilon c_{0}^{3}}{8}\right) t+3 \phi_{0}\right]-\right. \\
& \left.-\frac{1}{32} \cos 3 \phi_{0} \cos t+3\left(\frac{1}{8} \sin \phi_{0}+\frac{1}{32} \sin 3 \phi_{0}\right) \sin t\right]
\end{aligned}
$$

The method of multiple scales has replaced the original ordinary differential equation with a series of partial differential equations. The solution found by multiple scales is uniform, since it does not contain any false secular terms. The technique constructs a uniform solution by finding how the "constants" of integration must vary (in a manner similar to the method of variation 


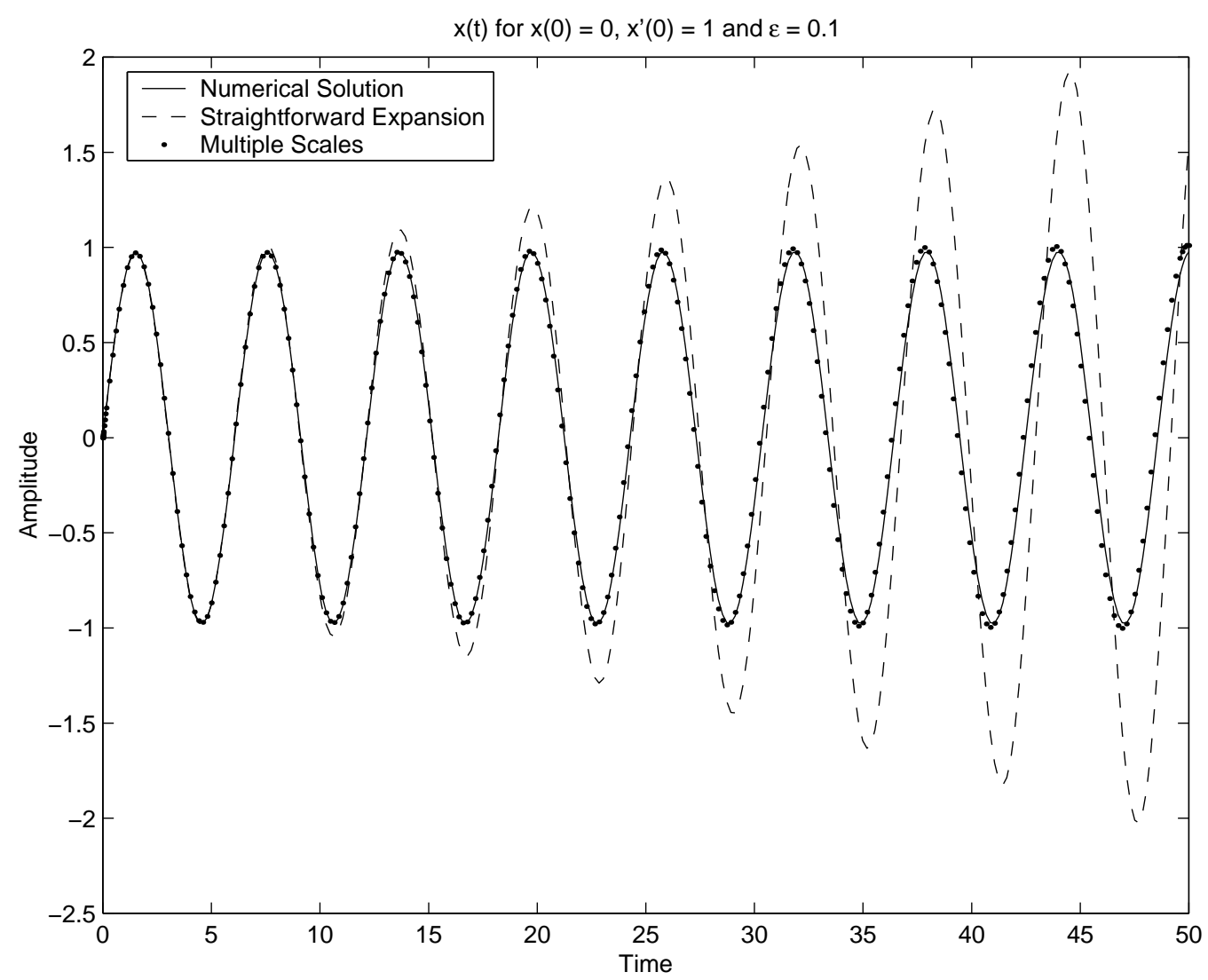

Figure 2.3: $x(t)$ Response for $\epsilon=0.1$

of parameters) in order to eliminate secular terms. This leads to systems of partial differential equations that describes the behavior of these parameters. These conditions, whatever the form, are sometimes called solvability conditions.

The results of the application of this perturbation method is compared with numerical integration of the original nonlinear ordinary differential equation in figures (2.3), (2.4) and (2.5). The plots show the amplitude response where the numerical integration is in solid, the straightforward expansion in dashed, and the multiple scales solution in dotted lines. All figures also use the initial conditions $x(0)=0$ and $\dot{x}(0)=1$ for varying values of $\epsilon$ and a time scale from 0 to 50 units. 


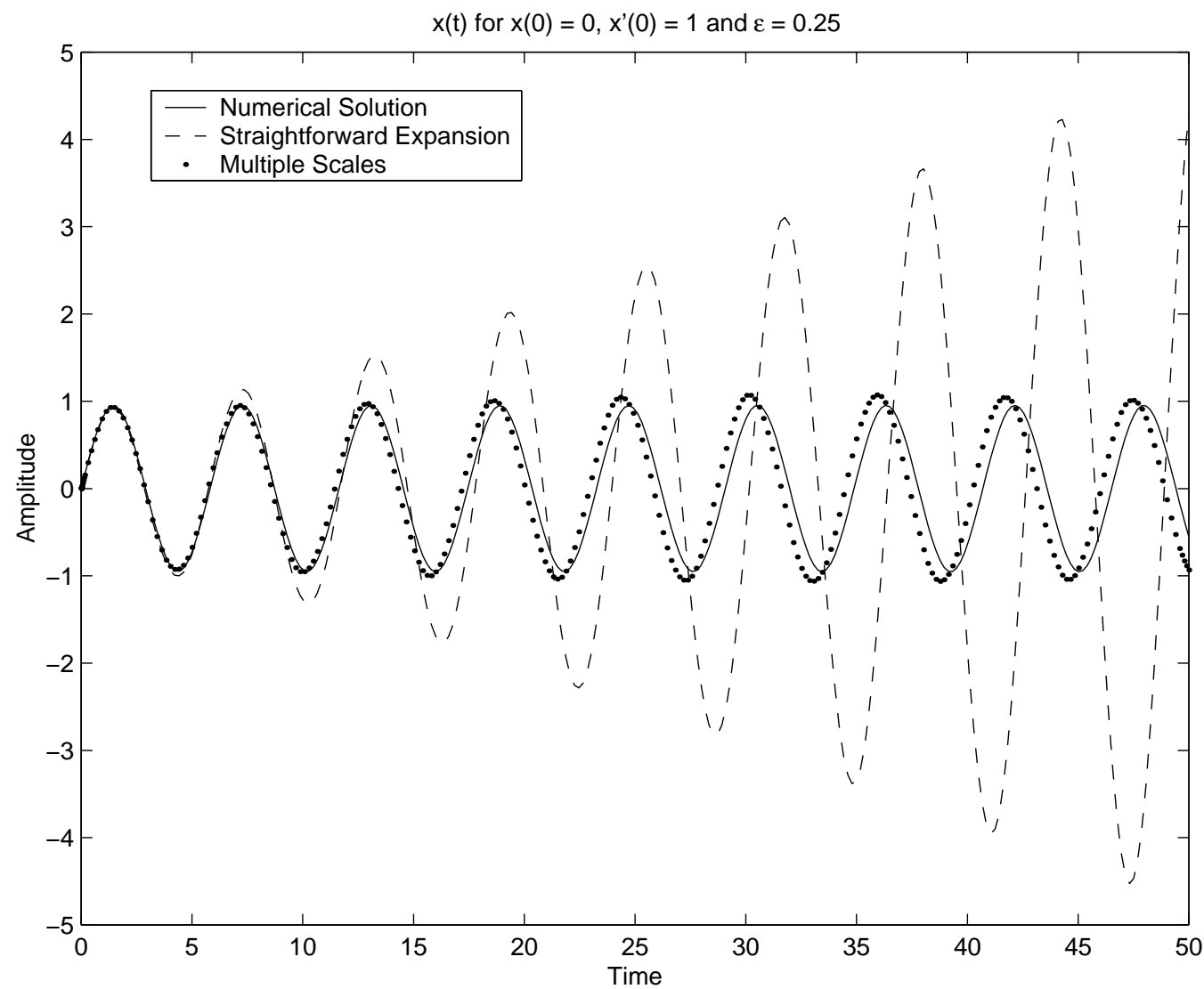

Figure 2.4: $x(t)$ Response for $\epsilon=0.25$ 


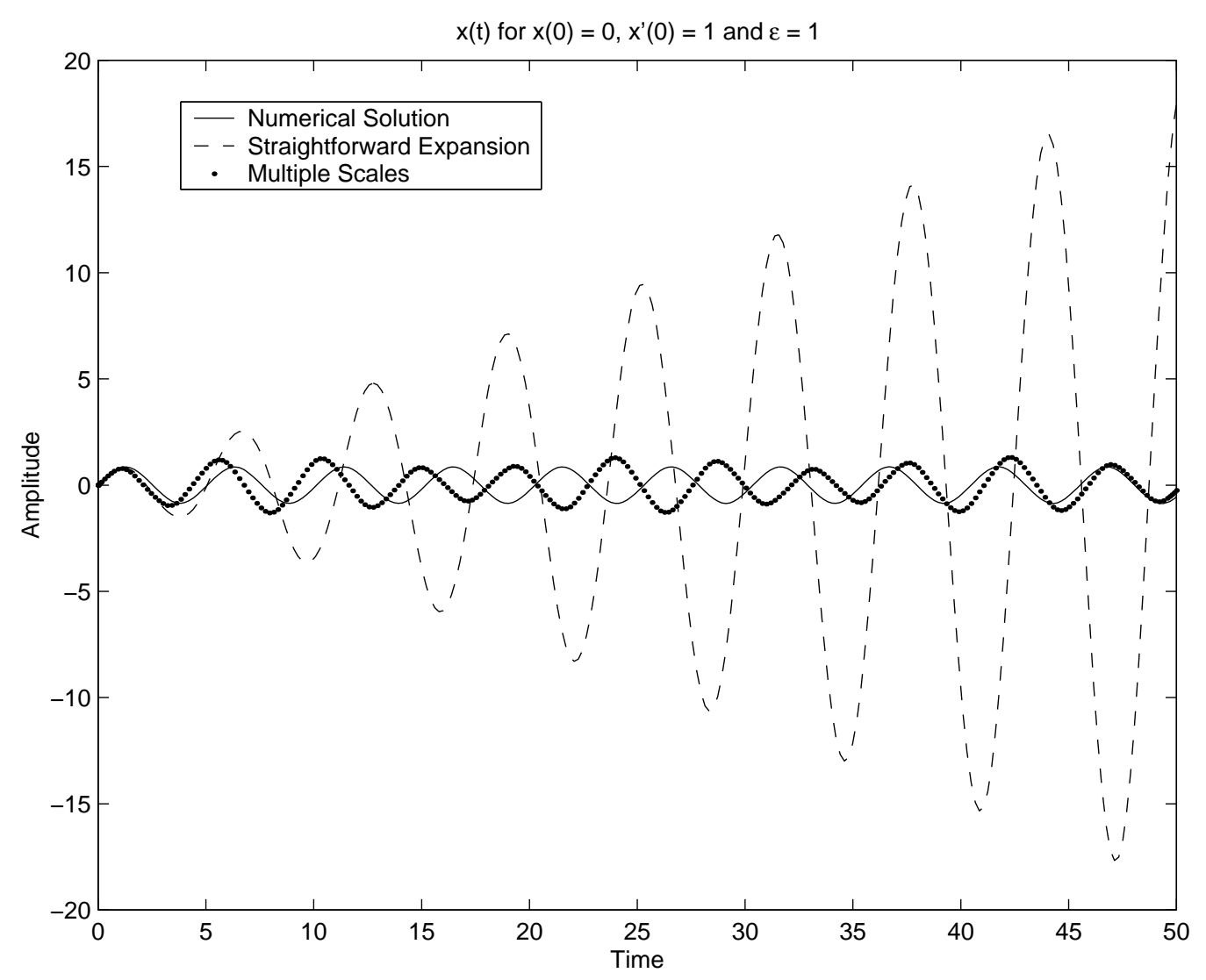

Figure 2.5: $x(t)$ Response for $\epsilon=1$

For $\epsilon=0.1$ the numerical integration, straightforward expansion, and multiple scales solution are essentially indistinguishable at the beginning, but as time increases the differences become apparent. The multiple scales solution remains close to the numerical integration, while the secular terms contained within the straightforward expansion begin to dominate at $t=\frac{1}{\epsilon}=10$ and the solution diverges. This divergence is further demonstrated in figures (2.4) and (2.5) for larger values of $\epsilon$. In these examples the straightforward expansion diverges sooner, and slight frequency differences are apparent in the numerical integration and multiple scales solution. The error between the multiple scales solution and numerical integration remains bounded, however, because the motion of each is bounded on its own.

In order to apply a perturbation method to a weakly nonlinear problem, it is first necessary to know the solution to the linear problem. The next chapter develops a linear form of the equations of motion and the solution is found my means of Laplace transforms. This solution will be needed in chapter (4) where a perturbation method is applied to a nonlinear form of the equations of motion in order to find an approximate solution. 


\section{Chapter 3}

\section{Linear Model}

This chapter will review the development of the linear relative motion equations. The orbital dynamics will be approximated by a Taylor series expansion of full nonlinear equations of motion about a circular reference orbit. This expansion is truncated after one term, leading to a system of linear, constant coefficient, ordinary differential equations. Non-dimensional state variables are introduced to the problem. Laplace transforms are used to solve this linear system, and the solution is presented in several useful forms.

\subsection{Equations of Motion}

As derived in chapter 2, the equations of motion in spherical coordinates, but now expressed in state variable form, are

$$
\begin{aligned}
\dot{r} & =v_{r} \\
\dot{\theta} & =\omega_{\theta} \\
\dot{\phi} & =\omega_{\phi} \\
\dot{v}_{r} & =r \omega_{\phi}^{2}+r \omega_{\theta}^{2} \cos ^{2} \phi-\frac{\mu}{r^{2}} \\
\dot{\omega}_{\theta} & =-\frac{2 v_{r} \omega_{\theta}}{r}+2 \omega_{\theta} \omega_{\phi} \tan \phi \\
\dot{\omega}_{\phi} & =-\frac{2 v_{r} \omega_{\phi}}{r}-\omega_{\theta}^{2} \sin \phi \cos \phi
\end{aligned}
$$

A linear representation of the dynamics can be found by using a Taylor expansion of equations $(3.4-3.6)$ (equations $(3.1-3.3)$ being already linear) with the reference conditions

$$
\begin{aligned}
r_{\text {ref }} & =r_{0} \\
\phi_{\text {ref }} & =0
\end{aligned}
$$




$$
\begin{aligned}
v_{r_{\text {ref }}} & =0 \\
\omega_{\theta_{\text {ref }}} & =\omega_{0} \\
\omega_{\phi_{\text {ref }}} & =0
\end{aligned}
$$

and only keeping the linear terms. These reference conditions are consistent with a circular orbit in the r- $\theta$ plane of radius $r_{0}$ and mean motion $\omega_{0}$. Note that the variable $\theta$ does not appear in the equations of motion. Variables that have no effect upon the dynamics of a system are often called ignorable or cyclic variables. Since $\theta$ is an ignorable variable, no reference condition is needed to expand the equations of motion. It is necessary to introduce a new set of variables $\Delta r, \Delta \theta, \Delta \phi, \Delta v_{r}, \Delta \omega_{\theta}$ and $\Delta \omega_{\phi}$ where

$$
\begin{aligned}
\Delta r & =r-r_{0} \\
\Delta \theta & =\theta-\theta_{0} \\
\Delta \phi & =\phi \\
\Delta v_{r} & =v_{r} \\
\Delta \omega_{\theta} & =\omega_{\theta}-\omega_{0} \\
\Delta \omega_{\phi} & =\omega_{\phi}
\end{aligned}
$$

A Taylor expansion of equation (3.4) about these conditions, and only keeping the linear terms results in

$$
\begin{gathered}
\dot{v}_{r}=\left[\dot{v}_{r}\right]_{r e f}+\Delta r\left[\frac{\partial \dot{v}_{r}}{\partial r}\right]_{r e f}+\Delta \theta\left[\frac{\partial \dot{v}_{r}}{\partial \theta}\right]_{r e f}+\Delta \phi\left[\frac{\partial \dot{v}_{r}}{\partial \phi}\right]_{r e f}+ \\
+\Delta v_{r}\left[\frac{\partial \dot{v}_{r}}{\partial v_{r}}\right]_{r e f}+\Delta \omega_{\theta}\left[\frac{\partial \dot{v}_{r}}{\partial \omega_{\theta}}\right]_{r e f}+\Delta \omega_{\phi}\left[\frac{\partial \dot{v}_{r}}{\partial \omega_{\phi}}\right]_{r e f}
\end{gathered}
$$

where

$$
\begin{aligned}
{\left[\dot{v}_{r}\right]_{r e f} } & =r_{0} \omega_{0}^{2}-\frac{\mu}{r_{0}^{2}} \\
& =r_{0} \omega_{0}^{2}-r_{0} \omega_{0}^{2} \\
& =0 \\
{\left[\frac{\partial \dot{v}_{r}}{\partial r}\right]_{r e f} } & =\left[\omega_{\phi}^{2}+\omega_{\theta}^{2} \cos ^{2} \phi+2 \frac{\mu}{r^{3}}\right]_{r e f} \\
& =3 \omega_{0}^{2} \\
{\left[\frac{\partial \dot{v}_{r}}{\partial \theta}\right]_{r e f} } & =0
\end{aligned}
$$




$$
\begin{aligned}
{\left[\frac{\partial \dot{v}_{r}}{\partial \phi}\right]_{r e f} } & =\left[-2 r \omega_{\theta}^{2} \cos \phi \sin \phi\right]_{r e f} \\
& =0 \\
{\left[\frac{\partial \dot{v}_{r}}{\partial v_{r}}\right]_{r e f} } & =0 \\
{\left[\frac{\partial \dot{v}_{r}}{\partial \omega_{\theta}}\right]_{r e f} } & =\left[2 r \omega_{\theta} \cos ^{2} \phi\right]_{r e f} \\
& =2 r_{0} \omega_{0} \\
{\left[\frac{\partial \dot{v}_{r}}{\partial \omega_{\phi}}\right]_{r e f} } & =\left[2 r \omega_{\phi}\right]_{r e f} \\
& =0
\end{aligned}
$$

Similarly, expanding equation (3.5) results in

$$
\begin{aligned}
\dot{\omega}_{\theta}= & {\left[\dot{\omega}_{\theta}\right]_{r e f}+\Delta r\left[\frac{\partial \dot{\omega}_{\theta}}{\partial r}\right]_{r e f}+\Delta \theta\left[\frac{\partial \dot{\omega}_{\theta}}{\partial \theta}\right]_{r e f}+\Delta \phi\left[\frac{\partial \dot{\omega}_{\theta}}{\partial \phi}\right]_{r e f}+} \\
& +\Delta v_{r}\left[\frac{\partial \dot{\omega}_{\theta}}{\partial v_{r}}\right]_{r e f}+\Delta \omega_{\theta}\left[\frac{\partial \dot{\omega}_{\theta}}{\partial \omega_{\theta}}\right]_{r e f}+\Delta \omega_{\phi}\left[\frac{\partial \dot{\omega}_{\theta}}{\partial \omega_{\phi}}\right]_{r e f}
\end{aligned}
$$

where

$$
\begin{aligned}
{\left[\dot{\omega}_{\theta}\right]_{r e f} } & =\left[-\frac{2 v_{r} \omega_{\theta}}{r}+2 \omega_{\theta} \omega_{\phi} \tan \phi\right]_{r e f} \\
& =0 \\
{\left[\frac{\partial \dot{\omega}_{\theta}}{\partial r}\right]_{r e f} } & =\left[\frac{2 v_{r} \omega_{\theta}}{r^{2}}\right]_{r e f} \\
& =0 \\
{\left[\frac{\partial \dot{\omega}_{\theta}}{\partial \theta}\right]_{r e f} } & =0 \\
{\left[\frac{\partial \dot{\omega}_{\theta}}{\partial \phi}\right]_{r e f} } & =\left[2 \omega_{\theta} \omega_{\phi} \sec ^{2} \phi\right]_{r e f} \\
& =0
\end{aligned}
$$




$$
\begin{aligned}
{\left[\frac{\partial \dot{\omega}_{\theta}}{\partial v_{r}}\right]_{r e f} } & =\left[-\frac{2 \omega_{\theta}}{r}\right] \\
& =-\frac{2 \omega_{0}}{r_{0}} \\
{\left[\frac{\partial \dot{\omega}_{\theta}}{\partial \omega_{\theta}}\right]_{r e f} } & =\left[-\frac{2 v_{r}}{r}+2 \omega_{\phi} \tan \phi\right]_{r e f} \\
& =0 \\
{\left[\frac{\partial \dot{\omega}_{\theta}}{\partial \omega_{\phi}}\right]_{r e f} } & =\left[2 \omega_{\theta} \tan \phi\right]_{r e f} \\
& =0
\end{aligned}
$$

Lastly, the expansion of equation (3.6) is

$$
\begin{gathered}
\dot{\omega}_{\phi}=\left[\dot{\omega}_{\phi}\right]_{r e f}+\Delta r\left[\frac{\partial \dot{\omega}_{\phi}}{\partial r}\right]_{r e f}+\Delta \theta\left[\frac{\partial \dot{\omega}_{\phi}}{\partial \theta}\right]_{r e f}+\Delta \phi\left[\frac{\partial \dot{\omega}_{\phi}}{\partial \phi}\right]_{r e f}+ \\
+\Delta v_{r}\left[\frac{\partial \dot{\omega}_{\phi}}{\partial v_{r}}\right]_{r e f}+\Delta \omega_{\theta}\left[\frac{\partial \dot{\omega}_{\phi}}{\partial \omega_{\theta}}\right]_{r e f}+\Delta \omega_{\phi}\left[\frac{\partial \dot{\omega}_{\phi}}{\partial \omega_{\phi}}\right]_{r e f}
\end{gathered}
$$

where

$$
\begin{aligned}
{\left[\dot{\omega}_{\phi}\right]_{r e f} } & =\left[-\frac{2 v_{r} \omega_{\phi}}{r}-\omega_{\theta}^{2} \sin \phi \cos \phi\right]_{r e f} \\
& =0 \\
{\left[\frac{\partial \dot{\omega}_{\phi}}{\partial r}\right]_{r e f} } & =\left[\frac{2 v_{r} \omega_{\phi}}{r^{2}}\right]_{r e f} \\
& =0 \\
{\left[\frac{\partial \dot{\omega}_{\phi}}{\partial \theta}\right]_{r e f} } & =0 \\
{\left[\frac{\partial \dot{\omega}_{\phi}}{\partial \phi}\right]_{r e f} } & =\left[-\omega_{\theta}^{2} \cos ^{2} \phi+\omega_{\theta}^{2} \sin ^{2} \phi\right]_{r e f} \\
{\left[\frac{\partial \dot{\omega}_{\phi}}{\partial v_{r}}\right]_{r e f} } & =-\omega_{0}^{2}
\end{aligned}
$$




$$
\begin{aligned}
& =0 \\
{\left[\frac{\partial \dot{\omega}_{\phi}}{\partial \omega_{\theta}}\right]_{r e f} } & =\left[-2 \omega_{\theta} \sin \phi \cos \phi\right]_{r e f} \\
& =0 \\
{\left[\frac{\partial \dot{\omega}_{\phi}}{\partial \omega_{\phi}}\right]_{r e f} } & =\left[-\frac{2 v_{r}}{r}\right]_{r e f} \\
& =0
\end{aligned}
$$

Assembling equations (3.18), (3.26) and (3.34) results in

$$
\begin{aligned}
\Delta \dot{r} & =\Delta v_{r} \\
\Delta \dot{\theta} & =\Delta \omega_{\theta} \\
\Delta \dot{\phi} & =\Delta \omega_{\phi} \\
\Delta \dot{v}_{r} & =3 \omega_{0}^{2} \Delta r+2 r_{0} \omega_{0} \Delta \omega_{\theta} \\
\Delta \dot{\omega}_{\theta} & =-\frac{2 \omega_{0}}{r_{0}} \Delta v_{r} \\
\Delta \dot{\omega}_{\phi} & =-\omega_{0}^{2} \Delta \phi
\end{aligned}
$$

Note that the out-of-plane variable, $\phi$, has decoupled from the in-plane variables, $r$ and $\theta$. This means that the out-of-plane motion may be examined independently of the in-plane motion. Also, since $\theta$ is ignorable, the solution of (3.43) may be found separately from the solution of the rest of the in plane variables in equations (3.42), (3.45) and (3.46). It is convenient to non-dimensionalize the problem by introducing the variables

$$
\begin{aligned}
\Delta \hat{r} & =\frac{\Delta r}{r_{0}} \\
\Delta \hat{\theta} & =\Delta \theta \\
\Delta \hat{\phi} & =\Delta \phi
\end{aligned}
$$

and the non-dimensional time variable

$$
\tau=\omega_{0} t
$$

so that

$$
\frac{d(\cdot)}{d t}=\omega_{0} \frac{d(\cdot)}{d \tau}
$$

Using this new differentiation rule, the non-dimensional velocity variables become

$$
\Delta \hat{v}_{r}=\frac{\Delta v_{r}}{r_{0} \omega_{0}}
$$




$$
\begin{aligned}
\Delta \hat{\omega}_{\theta} & =\frac{\Delta \omega_{\theta}}{\omega_{0}} \\
\Delta \hat{\omega}_{\phi} & =\frac{\Delta \omega_{\phi}}{\omega_{0}}
\end{aligned}
$$

Assembling the non-dimensional equations of motion in matrix form, breaking up the uncoupled system and using the notation $(\cdot)^{\prime}$ to mean differentiation with respect to the nondimensional time variable $\tau$ results in

$$
\begin{aligned}
\left(\begin{array}{c}
\Delta \hat{r}^{\prime} \\
\Delta \hat{v}_{r}^{\prime} \\
\Delta \hat{\omega}_{\theta}^{\prime}
\end{array}\right) & =\left[\begin{array}{ccc}
0 & 1 & 0 \\
3 & 0 & 2 \\
0 & -2 & 0
\end{array}\right]\left(\begin{array}{c}
\Delta \hat{r} \\
\Delta \hat{v}_{r} \\
\Delta \hat{\omega}_{\theta}
\end{array}\right) \\
\Delta \hat{\theta}^{\prime} & =\Delta \hat{\omega}_{\theta} \\
\left(\begin{array}{c}
\Delta \hat{\phi}^{\prime} \\
\Delta \hat{\omega}_{\phi}^{\prime}
\end{array}\right) & =\left[\begin{array}{cc}
0 & 1 \\
-1 & 0
\end{array}\right]\left(\begin{array}{c}
\Delta \hat{\phi} \\
\Delta \hat{\omega}_{\phi}
\end{array}\right)
\end{aligned}
$$

Equations (3.56), (3.57) and (3.58) are the linearized form of the equations of motion represented in spherical coordinates. Overall, it is a sixth order system of ordinary differential equations, but due to decoupling of motion and an ignorable coordinate, this set of equations is reduced to a third order system, a first order system and a second order system. It is the subject of the next section to find the solutions of these equations.

\subsection{Solution}

Equations (3.56) through (3.58) are easily solved by means of Laplace transforms. A detailed description of the Laplace transform is given in reference [29]. For this process it is more convenient to write the equations of motion as

$$
\begin{aligned}
\Delta \hat{r}^{\prime \prime}-3 \Delta \hat{r}-2 \Delta \hat{\theta}^{\prime} & =0 \\
\Delta \hat{\theta}^{\prime \prime}+2 \Delta \hat{r}^{\prime} & =0 \\
\Delta \hat{\phi}^{\prime \prime}+\Delta \hat{\phi} & =0
\end{aligned}
$$

with initial conditions

$$
\begin{aligned}
\Delta \hat{r}(0) & =\Delta \hat{r}_{00} \\
\Delta \hat{\theta}(0) & =\Delta \hat{\theta}_{00} \\
\Delta \hat{\phi}(0) & =\Delta \hat{\phi}_{00} \\
\Delta \hat{v}_{r}(0) & =\Delta \hat{v}_{r_{00}} \\
\Delta \hat{\omega}_{\theta}(0) & =\Delta \hat{\omega}_{\theta_{00}} \\
\Delta \hat{\omega}_{\phi}(0) & =\Delta \hat{\omega}_{\phi_{00}}
\end{aligned}
$$


If the Laplace transform of $\Delta \hat{r}$ and $\Delta \hat{\theta}$ is $\Delta \hat{R}$ and $\Delta \hat{\Theta}$, respectively, then the Laplace transform of in-plane differential equations in matrix form is

$$
\left[\begin{array}{cc}
s^{2}-3 & -2 s \\
2 s & s^{2}
\end{array}\right]\left(\begin{array}{c}
\Delta \hat{R} \\
\Delta \hat{\Theta}
\end{array}\right)=\left(\begin{array}{c}
s \Delta \hat{r}_{00}+\Delta \hat{v}_{00}-2 \Delta \hat{v}_{00} \\
s \Delta \hat{\theta}_{00}+\Delta \hat{\omega}_{\theta_{00}}+2 \Delta \hat{r}_{00}
\end{array}\right)
$$

Inverting the matrix to solve for $\Delta \hat{R}$ and $\Delta \hat{\Theta}$ results in

$$
\begin{aligned}
\left(\begin{array}{c}
\Delta \hat{R} \\
\Delta \hat{\Theta}
\end{array}\right) & =\frac{1}{s^{2}\left(s^{2}+1\right)}\left[\begin{array}{cc}
s^{2} & 2 s \\
-2 s & s^{2}-3
\end{array}\right]\left(\begin{array}{c}
s \Delta \hat{r}_{00}+\Delta \hat{v}_{r_{00}}-2 \Delta \hat{\omega}_{\theta_{00}} \\
s \Delta \hat{\theta}_{00}+\Delta \hat{\omega}_{\theta_{00}}+2 \Delta \hat{r}_{00}
\end{array}\right) \\
& =\frac{1}{s^{2}\left(s^{2}+1\right)}\left(\begin{array}{c}
s^{3} \Delta \hat{r}_{00}+s^{2} \Delta \hat{v}_{r_{00}}+2 s\left(\Delta \hat{\omega}_{000}+2 \Delta \hat{r}_{00}\right) \\
s^{3} \Delta \hat{\theta}_{00}+s^{2} \Delta \hat{\omega}_{\theta_{00}}+s\left(\Delta \hat{\theta}_{00}-2 \Delta \hat{v}_{r_{00}}\right)-3 \Delta \hat{\omega}_{\theta_{00}}-6 \Delta \hat{r}_{00}
\end{array}\right)
\end{aligned}
$$

Using the notation $\mathcal{L}^{-1}$ for the inverse Laplace transform,

$$
\begin{aligned}
\mathcal{L}^{-1}\left[\frac{s^{3}}{s^{2}\left(s^{2}+1\right)}\right] & =\cos \tau \\
\mathcal{L}^{-1}\left[\frac{s^{2}}{s^{2}\left(s^{2}+1\right)}\right] & =\sin \tau \\
\mathcal{L}^{-1}\left[\frac{s}{s^{2}\left(s^{2}+1\right)}\right] & =1-\cos \tau \\
\mathcal{L}^{-1}\left[\frac{1}{s^{2}\left(s^{2}+1\right)}\right] & =\tau-\sin \tau
\end{aligned}
$$

Substituting equations $(3.71-3.74)$ into $(3.70$ gives the result

$$
\begin{aligned}
\Delta \hat{r}(\tau)= & 4 \Delta \hat{r}_{00}+2 \Delta \hat{\omega}_{\theta_{00}}-\left(3 \Delta \hat{r}_{00}+2 \Delta \hat{\omega}_{\theta_{00}}\right) \cos \tau+\Delta \hat{v}_{r_{00}} \sin \tau \\
\Delta \hat{\theta}(\tau)= & \Delta \hat{\theta}_{00}-2 \Delta \hat{v}_{r_{00}}-\left(6 \Delta \hat{r}_{00}+3 \Delta \hat{\omega}_{\theta_{00}}\right) \tau+2 \Delta \hat{v}_{r_{00}} \cos \tau+ \\
& +\left(6 \Delta \hat{v}_{r_{00}}+4 \Delta \hat{\omega}_{\theta_{00}}\right) \sin \tau
\end{aligned}
$$

and the expression for $\Delta \hat{v}_{r}$ and $\Delta \hat{\omega}_{\theta}$ are simply the derivatives of equations (3.75) and (3.76):

$$
\begin{aligned}
\Delta \hat{v}_{r}(\tau) & =\left(3 \Delta \hat{r}_{00}+2 \Delta \hat{\omega}_{\theta_{00}}\right) \sin \tau+\Delta \hat{v}_{r_{00}} \cos \tau \\
\Delta \hat{\omega}_{\theta}(\tau) & =-6 \Delta \hat{r}_{00}-3 \Delta \hat{\omega}_{\theta_{00}}-2 \Delta \hat{v}_{r_{00}} \sin \tau+\left(6 \Delta \hat{v}_{r_{00}}+4 \Delta \hat{\omega}_{\theta_{00}}\right) \cos \tau
\end{aligned}
$$

The out-of-plane equation is the same form as that of a linear mass-spring system with a natural frequency of 1 . The solution of this type of system is well known and takes the form

$$
\begin{aligned}
\Delta \hat{\phi}(\tau) & =\Delta \hat{\phi}_{00} \cos \tau+\Delta \hat{\omega}_{\phi_{00}} \sin \tau \\
\Delta \hat{\omega}_{\phi}(\tau) & =-\Delta \hat{\phi}_{00} \sin \tau+\Delta \hat{\omega}_{\phi_{00}} \cos \tau
\end{aligned}
$$


The solution may also be written in several other forms which will be useful in later sections. One is the state transition matrix form:

$$
\left(\begin{array}{c}
\Delta \hat{r} \\
\Delta \hat{\theta} \\
\Delta \hat{\phi} \\
\Delta \hat{v}_{r} \\
\Delta \hat{\omega}_{\theta} \\
\Delta \hat{\omega}_{\phi}
\end{array}\right)=\left[\begin{array}{cccccc}
4-3 \cos \tau & 0 & 0 & \sin \tau & 2(1-\cos \tau) & 0 \\
-6(\tau-\sin \tau) & 1 & 0 & 2 \cos \tau & 4 \sin \tau-3 \tau & 0 \\
0 & 0 & \cos \tau & 0 & 0 & \sin \tau \\
3 \sin \tau & 0 & 0 & \cos \tau & 2 \sin \tau & 0 \\
-6(1-\cos \tau) & 0 & 0 & -2 \sin \tau & 4 \cos \tau-3 & 0 \\
0 & 0 & -\sin \tau & 0 & 0 & \cos \tau
\end{array}\right]\left(\begin{array}{c}
\Delta \hat{r}_{00} \\
\Delta \hat{\theta}_{00} \\
\Delta \hat{\phi}_{00} \\
\Delta \hat{v}_{r_{00}} \\
\Delta \hat{\omega}_{\theta_{00}} \\
\Delta \hat{\omega}_{\phi_{00}}
\end{array}\right)
$$

Another form is

$$
\begin{aligned}
& \Delta \hat{r}=a \cos (\tau+\alpha)+b \\
& \Delta \hat{\theta}=-2 a \sin (\tau+\alpha)-\frac{3}{2} b \tau+c \\
& \Delta \hat{\phi}=d \cos (\tau+\delta)
\end{aligned}
$$

The constants $a, b, c, d, \alpha$ and $\delta$ may be found by expanding equations $(3.82-3.84)$ with trigonometric identities and comparing with equations (3.75), (3.76) and (3.79).

$$
\begin{aligned}
& \Delta \hat{r}=a \cos \alpha \cos \tau-a \sin \alpha \sin \tau+b \\
& \Delta \hat{\theta}=-2 a \cos \alpha \sin \tau-2 a \sin \alpha \cos \tau-\frac{3}{2} b \tau+c \\
& \Delta \hat{\phi}=d \cos \delta \cos \tau-d \sin \delta \sin \tau
\end{aligned}
$$

which leads to

$$
\begin{aligned}
a \cos \alpha & =-3 \Delta \hat{r}_{00}-2 \Delta \hat{\omega}_{\theta_{00}} \\
a \sin \alpha & =-\Delta \hat{v}_{r_{00}} \\
b & =4 \Delta \hat{r}_{00}+2 \Delta \hat{\omega}_{\theta_{00}} \\
c & =\Delta \hat{\theta}_{00}-2 \Delta \hat{v}_{r_{00}} \\
d \cos \delta & =\Delta \hat{\phi}_{00} \\
d \sin \delta & =\Delta \hat{\omega}_{\phi_{00}}
\end{aligned}
$$

Squaring and adding equations (3.88) and (3.89) as well as (3.92) and (3.93) results in

$$
\begin{aligned}
a^{2} & =\left(3 \Delta \hat{r}_{00}+2 \Delta \hat{\omega}_{\theta_{00}}\right)^{2}+\Delta \hat{v}_{r_{00}}^{2} \\
d^{2} & =\Delta \hat{\phi}_{00}^{2}+\Delta \hat{\omega}_{\phi_{00}}^{2} \\
\sin \alpha & =-\frac{\Delta \hat{v}_{r_{00}}}{a}
\end{aligned}
$$




$$
\begin{aligned}
\cos \alpha & =-\frac{3 \Delta \hat{r}_{00}+2 \Delta \hat{\omega}_{\theta_{00}}}{a} \\
\sin \delta & =-\frac{\Delta \hat{\omega}_{\phi_{00}}}{d} \\
\cos \delta & =\frac{\Delta \hat{\phi}_{00}}{d}
\end{aligned}
$$

Another form of the solution may be found by introducing the complex variables

$$
\begin{aligned}
A & =\frac{1}{2} a \mathrm{e}^{i \alpha} \\
B & =b \\
C & =c \\
D & =\frac{1}{2} d \mathrm{e}^{i \delta}
\end{aligned}
$$

where $a, b, c, d, \alpha$ and $\delta$ are as found above and $i=\sqrt{-1}$. Using these new variables, the solution may be written as

$$
\begin{aligned}
& \Delta \hat{r}=A \mathrm{e}^{i \tau}+\bar{A} \mathrm{e}^{-i \tau}+B \\
& \Delta \hat{\theta}=2 i A \mathrm{e}^{i \tau}-2 i \bar{A} \mathrm{e}^{-i \tau}-\frac{3}{2} B \tau+C \\
& \Delta \hat{\phi}=D \mathrm{e}^{i \tau}+\bar{D} \mathrm{e}^{-i \tau}
\end{aligned}
$$

where $\bar{A}$ and $\bar{D}$ are the complex conjugates of $A$ and $D$.

\subsection{Relative Position and Velocity Vectors}

In order to complete the problem it is necessary to express the solution in the form of relative position and relative velocity vectors. Using the coordinate systems shown below, the nondimensional relative position vector, $\hat{\mathbf{R}}$, may be written as

$$
\hat{\mathbf{R}}=(1+\Delta \hat{r}) \mathbf{e}_{r_{2}}-\mathbf{e}_{r}
$$

where $\mathbf{e}_{r_{2}}, \mathbf{e}_{\theta_{2}}$ and $\mathbf{e}_{\phi_{2}}$ are the unit vectors of the coordinate system with $\mathbf{e}_{r_{2}}$ aligned with the position vector of the satellite and $\mathbf{e}_{r}, \mathbf{e}_{\theta}$ and $\mathbf{e}_{\phi}$ are the unit vectors of the coordinate system aligned with the reference orbit. Vectors $\mathbf{e}_{r_{2}}, \mathbf{e}_{\theta_{2}}$ and $\mathbf{e}_{\phi_{2}}$ may be expressed in terms of $\mathbf{e}_{r}, \mathbf{e}_{\theta}$ and $\mathbf{e}_{\phi}$ by a series of two simple coordinate transformations: first a rotation of angle $\Delta \hat{\theta}$ about $\mathbf{e}_{\phi}$ to align the coordinate system with an intermediate system $\mathbf{e}_{r_{1}}, \mathbf{e}_{\theta_{1}}$ and $\mathbf{e}_{\phi_{1}}$, and then a rotation of angle $-\Delta \hat{\phi}$ about the $\mathbf{e}_{\theta_{1}}$ axis to align the intermediate system with $\mathbf{e}_{r_{2}}, \mathbf{e}_{\theta_{2}}$ and $\mathbf{e}_{\phi_{2}}$. In matrix form this operation may be written as

$$
\left(\begin{array}{c}
\mathbf{e}_{r_{2}} \\
\mathbf{e}_{\theta_{2}} \\
\mathbf{e}_{\phi_{2}}
\end{array}\right)=\left[\begin{array}{ccc}
\cos \Delta \hat{\phi} & 0 & \sin \Delta \hat{\phi} \\
0 & 1 & 0 \\
-\sin \Delta \hat{\phi} & 0 & \cos \Delta \hat{\phi}
\end{array}\right]\left[\begin{array}{ccc}
\cos \Delta \hat{\theta} & \sin \Delta \hat{\theta} & 0 \\
-\sin \Delta \hat{\theta} & \cos \Delta \hat{\theta} & 0 \\
0 & 0 & 1
\end{array}\right]\left(\begin{array}{c}
\mathbf{e}_{r} \\
\mathbf{e}_{\theta} \\
\mathbf{e}_{\phi}
\end{array}\right)
$$




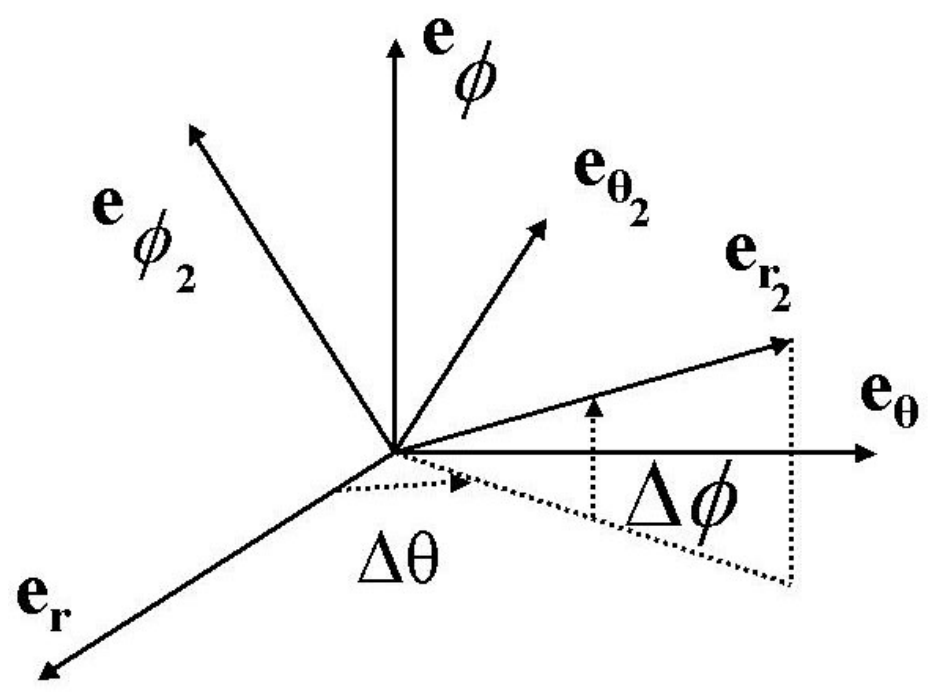

Figure 3.1: Relative Spherical Coordinate System

$$
=\left[\begin{array}{ccc}
\cos \Delta \hat{\phi} \cos \Delta \hat{\theta} & \cos \Delta \hat{\phi} \sin \Delta \hat{\theta} & \sin \Delta \hat{\phi} \\
-\sin \Delta \hat{\theta} & \cos \Delta \hat{\theta} & 0 \\
-\sin \Delta \hat{\phi} \cos \Delta \hat{\theta} & -\sin \Delta \hat{\theta} \sin \Delta \hat{\theta} \cos \Delta \hat{\phi}
\end{array}\right]\left(\begin{array}{c}
\mathbf{e}_{r} \\
\mathbf{e}_{\theta} \\
\mathbf{e}_{\phi}
\end{array}\right)
$$

Therefore, the relative position vector may be written as

$$
\hat{\mathbf{R}}=[(1+\Delta \hat{r}) \cos \Delta \hat{\phi} \cos \Delta \hat{\theta}-1] \mathbf{e}_{r}+(1+\Delta \hat{r}) \cos \Delta \hat{\phi} \sin \Delta \hat{\theta} \mathbf{e}_{\theta}+(1+\Delta \hat{r}) \sin \Delta \hat{\phi} \mathbf{e}_{\phi}
$$

Similarly to chapter (2), the derivatives of the unit vectors aligned with the reference orbit are

$$
\begin{aligned}
\mathbf{e}_{r}^{\prime} & =\mathbf{e}_{\theta} \\
\mathbf{e}_{\theta}^{\prime} & =-\mathbf{e}_{r} \\
\mathbf{e}_{\phi}^{\prime} & =0
\end{aligned}
$$

so that the relative velocity vector is

$$
\begin{aligned}
\hat{\mathbf{V}}= & {\left[\Delta \hat{v}_{r} \cos \Delta \hat{\phi} \cos \Delta \hat{\theta}-(1+\Delta \hat{r}) \Delta \hat{\omega}_{\phi} \sin \Delta \hat{\phi} \cos \Delta \hat{\theta}-\right.} \\
& \left.-(1+\Delta \hat{r}) \Delta \hat{\omega}_{\theta} \cos \Delta \hat{\phi} \sin \Delta \hat{\theta}-(1+\Delta \hat{r}) \cos \Delta \hat{\phi} \sin \Delta \hat{\theta}\right] \mathbf{e}_{r}+ \\
& +\left[\Delta \hat{v}_{r} \cos \Delta \hat{\phi} \sin \Delta \hat{\theta}-(1+\Delta \hat{r}) \Delta \hat{\omega}_{\phi} \sin \Delta \hat{\phi} \sin \Delta \hat{\theta}+\right. \\
& \left.+(1+\Delta \hat{r}) \Delta \hat{\omega}_{\theta} \cos \Delta \hat{\phi} \cos \Delta \hat{\theta}+(1+\Delta \hat{r}) \cos \Delta \hat{\phi} \cos \Delta \hat{\theta}-1\right] \mathbf{e}_{\theta}+ \\
& +\left[\Delta \hat{v}_{r} \sin \Delta \hat{\phi}+(1+\Delta \hat{r}) \Delta \hat{\omega}_{\phi} \cos \Delta \hat{\phi}\right] \mathbf{e}_{\phi}
\end{aligned}
$$


The absolute position and velocity of the satellite are simply

$$
\begin{aligned}
\hat{\mathbf{r}} & =\mathbf{e}_{r}+\hat{\mathbf{R}} \\
\hat{\mathbf{V}} & =\mathbf{e}_{\theta}+\hat{\mathbf{V}}
\end{aligned}
$$

The equations developed in this chapter, regardless of the form they are expressed in, are the solution to a linearized representation of the orbital motion. These solutions are known as Hill's equations or as the Clohessy-Wiltshire equations, although in spherical coordinates rather than the more traditional rectangular form used in [1] and [2]. 


\section{Chapter 4}

\section{Nonlinear Model}

This chapter develops the analytical solution of nonlinear relative motion equations. A Taylor series expansion of the orbital dynamics is used to approximate the full nonlinear equations of motion as in the previous chapter. Here the expansion will be truncated after secondorder terms, leading to a system of nonlinear ordinary differential equations. The same nondimensional state variables are introduced. The method of multiple scales is used to determine an approximate solution of the second-order equations of motion.

\subsection{Equations of Motion}

This section will develop a set of differential equations similar to that found in (3.59), (3.60) and (3.61), except that now they will contain second order terms, in other words, terms with quadratic nonlinearity. These equations will be developed in the same manner as the previous chapter, but now the Taylor expansion will be carried on to the next order. The same reference conditions will be used. The expansion of (3.4) is

$$
\begin{aligned}
\dot{v}_{r}= & {\left[\dot{v}_{r}\right]_{r e f}+\Delta r\left[\frac{\partial \dot{v}_{r}}{\partial r}\right]_{r e f}+\Delta \theta\left[\frac{\partial \dot{v}_{r}}{\partial \theta}\right]_{r e f}+\Delta \phi\left[\frac{\partial \dot{v}_{r}}{\partial \phi}\right]_{r e f}+\Delta v_{r}\left[\frac{\partial \dot{v}_{r}}{\partial v_{r}}\right]_{r e f}+} \\
& +\Delta \omega_{\theta}\left[\frac{\partial \dot{v}_{r}}{\partial \omega_{\theta}}\right]_{r e f}+\Delta \omega_{\phi}\left[\frac{\partial \dot{v}_{r}}{\partial \omega_{\phi}}\right]_{r e f}+\frac{1}{2} \Delta r^{2}\left[\frac{\partial^{2} \dot{v}_{r}}{\partial r^{2}}\right]_{r e f}+\Delta \theta \Delta r\left[\frac{\partial^{2} \dot{v}_{r}}{\partial \theta \partial r}\right]_{r e f}+ \\
& +\Delta \phi \Delta r\left[\frac{\partial^{2} \dot{v}_{r}}{\partial \phi \partial r}\right]_{r e f}+\Delta v_{r} \Delta r\left[\frac{\partial^{2} \dot{v}_{r}}{\partial v_{r} \partial r}\right]_{r e f}+\Delta \omega_{\theta} \Delta r\left[\frac{\partial^{2} \dot{v}_{r}}{\partial \omega_{\theta} \partial r}\right]_{r e f}+ \\
& +\Delta \omega_{\phi} \Delta r\left[\frac{\partial^{2} \dot{v}_{r}}{\partial \omega_{\phi} \partial r}\right]_{r e f}+\frac{1}{2} \Delta \theta^{2}\left[\frac{\partial^{2} \dot{v}_{r}}{\partial \theta^{2}}\right]_{r e f}+\Delta \phi \Delta \theta\left[\frac{\partial^{2} \dot{v}_{r}}{\partial \phi \partial \theta}\right]_{r e f}+ \\
& +\Delta v_{r} \Delta \theta\left[\frac{\partial^{2} \dot{v}_{r}}{\partial v_{r} \partial \theta}\right]_{r e f}+\Delta \omega_{\theta} \Delta \theta\left[\frac{\partial^{2} \dot{v}_{r}}{\partial \omega_{\theta} \partial \theta}\right]_{r e f}+\Delta \omega_{\phi} \Delta \theta\left[\frac{\partial^{2} \dot{v}_{r}}{\partial \omega_{\phi} \partial \theta}\right]_{r e f}+
\end{aligned}
$$




$$
\begin{aligned}
& +\frac{1}{2} \Delta \phi^{2}\left[\frac{\partial^{2} \dot{v}_{r}}{\partial \phi^{2}}\right]_{r e f}+\Delta v_{r} \Delta \phi\left[\frac{\partial^{2} \dot{v}_{r}}{\partial v_{r} \partial \phi}\right]_{r e f}+\Delta \omega_{\theta} \Delta \phi\left[\frac{\partial^{2} \dot{v}_{r}}{\partial \omega_{\theta} \partial \phi}\right]_{r e f}+ \\
& +\Delta \omega_{\phi} \Delta \phi\left[\frac{\partial^{2} \dot{v}_{r}}{\partial \omega_{\phi} \partial \phi}\right]_{r e f}+\frac{1}{2} \Delta v_{r}^{2}\left[\frac{\partial^{2} \dot{v}_{r}}{\partial v_{r}^{2}}\right]_{r e f}+\Delta \omega_{\theta} \Delta v_{r}\left[\frac{\partial^{2} \dot{v}_{r}}{\partial \omega_{\theta} \partial v_{r}}\right]_{r e f}+ \\
& +\Delta \omega_{\phi} \Delta v_{r}\left[\frac{\partial^{2} \dot{v}_{r}}{\partial \omega_{\phi} \partial v_{r}}\right]_{r e f}+\frac{1}{2} \Delta \omega_{\theta}^{2}\left[\frac{\partial^{2} \dot{v}_{r}}{\partial \omega_{\theta}^{2}}\right]_{r e f}+\Delta \omega_{\theta} \Delta \omega_{\phi}\left[\frac{\partial^{2} \dot{v}_{r}}{\partial \omega_{\phi} \partial \omega_{\theta}}\right]_{r e f}+ \\
& +\frac{1}{2} \Delta \omega_{\phi}^{2}\left[\frac{\partial^{2} \dot{v}_{r}}{\partial \omega_{\phi}^{2}}\right]_{r e f}
\end{aligned}
$$

The expansion of equation (3.5) is

$$
\begin{aligned}
\dot{\omega}_{\theta}= & {\left[\dot{\omega}_{\theta}\right]_{r e f}+\Delta r\left[\frac{\partial \dot{\omega}_{\theta}}{\partial r}\right]_{r e f}+\Delta \theta\left[\frac{\partial \dot{\omega}_{\theta}}{\partial \theta}\right]_{r e f}+\Delta \phi\left[\frac{\partial \dot{\omega}_{\theta}}{\partial \phi}\right]_{r e f}+\Delta v_{r}\left[\frac{\partial \dot{\omega}_{\theta}}{\partial v_{r}}\right]_{r e f}+} \\
& +\Delta \omega_{\theta}\left[\frac{\partial \dot{\omega}_{\theta}}{\partial \omega_{\theta}}\right]_{r e f}+\Delta \omega_{\phi}\left[\frac{\partial \dot{\omega}_{\theta}}{\partial \omega_{\phi}}\right]_{r e f}+\frac{1}{2} \Delta r^{2}\left[\frac{\partial^{2} \dot{\omega}_{\theta}}{\partial r^{2}}\right]_{r e f}+\Delta \theta \Delta r\left[\frac{\partial^{2} \dot{\omega}_{\theta}}{\partial \theta \partial r}\right]_{r e f}+ \\
& +\Delta \phi \Delta r\left[\frac{\partial^{2} \dot{\omega}_{\theta}}{\partial \phi \partial r}\right]_{r e f}+\Delta v_{r} \Delta r\left[\frac{\partial^{2} \dot{\omega}_{\theta}}{\partial v_{r} \partial r}\right]_{r e f}+\Delta \omega_{\theta} \Delta r\left[\frac{\partial^{2} \dot{\omega}_{\theta}}{\partial \omega_{\theta} \partial r}\right]_{r e f}+ \\
& +\Delta \omega_{\phi} \Delta r\left[\frac{\partial^{2} \dot{\omega}_{\theta}}{\partial \omega_{\phi} \partial r}\right]_{r e f}+\frac{1}{2} \Delta \theta^{2}\left[\frac{\partial^{2} \dot{\omega}_{\theta}}{\partial \theta^{2}}\right]_{r e f}+\Delta \phi \Delta \theta\left[\frac{\partial^{2} \dot{\omega}_{\theta}}{\partial \phi \partial \theta}\right]_{r e f}+ \\
& +\Delta v_{r} \Delta \theta\left[\frac{\partial^{2} \dot{\omega}_{\theta}}{\partial v_{r} \partial \theta}\right]_{r e f}+\Delta \omega_{\theta} \Delta \theta\left[\frac{\partial^{2} \dot{\omega}_{\theta}}{\partial \omega_{\theta} \partial \theta}\right]_{r e f}+\Delta \omega_{\phi} \Delta \theta\left[\frac{\partial^{2} \dot{\omega}_{\theta}}{\partial \omega_{\phi} \partial \theta}\right]_{r e f}+ \\
& +\frac{1}{2} \Delta \phi^{2}\left[\frac{\partial^{2} \dot{\omega}_{\theta}}{\partial \phi^{2}}\right]_{r e f}+\Delta v_{r} \Delta \phi\left[\frac{\partial^{2} \dot{\omega}_{\theta}}{\partial v_{r} \partial \phi}\right]_{r e f}+\Delta \omega_{\theta} \Delta \phi\left[\frac{\partial^{2} \dot{\omega}_{\theta}}{\partial \omega_{\theta} \partial \phi}\right]_{r e f}+ \\
& +\Delta \omega_{\phi} \Delta \phi\left[\frac{\partial^{2} \dot{\omega}_{\theta}}{\partial \omega_{\phi} \partial \phi}\right]_{r e f}+\frac{1}{2} \Delta v_{r}^{2}\left[\frac{\partial^{2} \dot{\omega}_{\theta}}{\partial v_{r}^{2}}\right]_{r e f}+\Delta \omega_{\theta} \Delta v_{r}\left[\frac{\partial^{2} \dot{\omega}_{\theta}}{\partial \omega_{\theta} \partial v_{r}}\right]_{r e f}+ \\
& +\Delta \omega_{\phi} \Delta v_{r}\left[\frac{\partial^{2} \dot{\omega}_{\theta}}{\partial \omega_{\phi} \partial v_{r}}\right]_{r e f}+\frac{1}{2} \Delta \omega_{\theta}^{2}\left[\frac{\partial^{2} \dot{\omega}_{\theta}}{\partial \omega_{\theta}^{2}}\right]_{r e f}+\Delta \omega_{\theta} \Delta \omega_{\phi}\left[\frac{\partial^{2} \dot{\omega}_{\theta}}{\partial \omega_{\phi} \partial \omega_{\theta}}\right]_{r e f}+ \\
& +\frac{1}{2} \Delta \omega_{\phi}^{2}\left[\frac{\partial^{2} \dot{\omega}_{\theta}}{\partial \omega_{\phi}^{2}}\right]_{r e f}
\end{aligned}
$$

Finally, the expansion of (3.6) is

$$
\begin{aligned}
\dot{\omega}_{\phi}= & {\left[\dot{\omega}_{\phi}\right]_{r e f}+\Delta r\left[\frac{\partial \dot{\omega}_{\phi}}{\partial r}\right]_{r e f}+\Delta \theta\left[\frac{\partial \dot{\omega}_{\phi}}{\partial \theta}\right]_{r e f}+\Delta \phi\left[\frac{\partial \dot{\omega}_{\phi}}{\partial \phi}\right]_{r e f}+\Delta v_{r}\left[\frac{\partial \dot{\omega}_{\phi}}{\partial v_{r}}\right]_{r e f}+} \\
& +\Delta \omega_{\theta}\left[\frac{\partial \dot{\omega}_{\phi}}{\partial \omega_{\theta}}\right]_{r e f}+\Delta \omega_{\phi}\left[\frac{\partial \dot{\omega}_{\phi}}{\partial \omega_{\phi}}\right]_{r e f}+\frac{1}{2} \Delta r^{2}\left[\frac{\partial^{2} \dot{\omega}_{\phi}}{\partial r^{2}}\right]_{r e f}+\Delta \theta \Delta r\left[\frac{\partial^{2} \dot{\omega}_{\phi}}{\partial \theta \partial r}\right]_{r e f}+ \\
& +\Delta \phi \Delta r\left[\frac{\partial^{2} \dot{\omega}_{\phi}}{\partial \phi \partial r}\right]_{r e f}+\Delta v_{r} \Delta r\left[\frac{\partial^{2} \dot{\omega}_{\phi}}{\partial v_{r} \partial r}\right]_{r e f}+\Delta \omega_{\theta} \Delta r\left[\frac{\partial^{2} \dot{\omega}_{\phi}}{\partial \omega_{\theta} \partial r}\right]_{r e f}+
\end{aligned}
$$




$$
\begin{aligned}
& +\Delta \omega_{\phi} \Delta r\left[\frac{\partial^{2} \dot{\omega}_{\phi}}{\partial \omega_{\phi} \partial r}\right]_{r e f}+\frac{1}{2} \Delta \theta^{2}\left[\frac{\partial^{2} \dot{\omega}_{\phi}}{\partial \theta^{2}}\right]_{r e f}+\Delta \phi \Delta \theta\left[\frac{\partial^{2} \dot{\omega}_{\phi}}{\partial \phi \partial \theta}\right]_{r e f}+ \\
& +\Delta v_{r} \Delta \theta\left[\frac{\partial^{2} \dot{\omega}_{\phi}}{\partial v_{r} \partial \theta}\right]_{r e f}+\Delta \omega_{\theta} \Delta \theta\left[\frac{\partial^{2} \dot{\omega}_{\phi}}{\partial \omega_{\theta} \partial \theta}\right]_{r e f}+\Delta \omega_{\phi} \Delta \theta\left[\frac{\partial^{2} \dot{\omega}_{\phi}}{\partial \omega_{\phi} \partial \theta}\right]_{r e f}+ \\
& +\frac{1}{2} \Delta \phi^{2}\left[\frac{\partial^{2} \dot{\omega}_{\phi}}{\partial \phi^{2}}\right]_{r e f}+\Delta v_{r} \Delta \phi\left[\frac{\partial^{2} \dot{\omega}_{\phi}}{\partial v_{r} \partial \phi}\right]_{r e f}+\Delta \omega_{\theta} \Delta \phi\left[\frac{\partial^{2} \dot{\omega}_{\phi}}{\partial \omega_{\theta} \partial \phi}\right]_{r e f}+ \\
& +\Delta \omega_{\phi} \Delta \phi\left[\frac{\partial^{2} \dot{\omega}_{\phi}}{\partial \omega_{\phi} \partial \phi}\right]_{r e f}+\frac{1}{2} \Delta v_{r}^{2}\left[\frac{\partial^{2} \dot{\omega}_{\phi}}{\partial v_{r}^{2}}\right]_{r e f}+\Delta \omega_{\theta} \Delta v_{r}\left[\frac{\partial^{2} \dot{\omega}_{\phi}}{\partial \omega_{\theta} \partial v_{r}}\right]_{r e f}+ \\
& +\Delta \omega_{\phi} \Delta v_{r}\left[\frac{\partial^{2} \dot{\omega}_{\phi}}{\partial \omega_{\phi} \partial v_{r}}\right]_{r e f}+\frac{1}{2} \Delta \omega_{\theta}^{2}\left[\frac{\partial^{2} \dot{\omega}_{\phi}}{\partial \omega_{\theta}^{2}}\right]_{r e f}+\Delta \omega_{\theta} \Delta \omega_{\phi}\left[\frac{\partial^{2} \dot{\omega}_{\phi}}{\partial \omega_{\phi} \partial \omega_{\theta}}\right]_{r e f}+ \\
& +\frac{1}{2} \Delta \omega_{\phi}^{2}\left[\frac{\partial^{2} \dot{\omega}_{\phi}}{\partial \omega_{\phi}^{2}}\right]_{r e f}
\end{aligned}
$$

The process of evaluating each of these terms is given in appendix (A). Assembling these terms leads to

$$
\begin{aligned}
\Delta \dot{r}= & \Delta v_{r} \\
\Delta \dot{\theta}= & \Delta \omega_{\theta} \\
\Delta \dot{\phi}= & \Delta \omega_{\phi} \\
\Delta \dot{v}_{r}= & 3 \omega_{0}^{2} \Delta r+2 r_{0} \omega_{0} \Delta \omega_{\theta}-\frac{3 \omega_{0}^{2}}{r_{0}} \Delta r^{2}+2 \omega_{0} \Delta r \Delta \omega_{\theta}- \\
& -r_{0} \omega_{0}^{2} \Delta \phi^{2}+r_{0} \Delta \omega_{\theta}^{2}+r_{0} \Delta \omega_{\phi}^{2} \\
\Delta \dot{\omega}_{\theta}= & -\frac{2 \omega_{0}}{r_{0}} \Delta v_{r}+\frac{2 \omega_{0}}{r_{0}^{2}} \Delta v_{r} \Delta r+2 \omega_{0} \Delta \omega_{\phi} \Delta \phi-\frac{2}{r_{0}} \Delta \omega_{\theta} \Delta v_{r} \\
\Delta \dot{\omega}_{\phi}= & -\omega_{0}^{2} \Delta \phi-2 \Delta \omega_{\theta} \Delta \phi-\frac{2}{r_{0}} \Delta \omega_{\phi} \Delta v_{r}
\end{aligned}
$$

Using the same non-dimensionalization as the previous chapter, and writing the equations as coupled systems of second order differential equations rather than in state variable form leads to

$$
\begin{aligned}
\Delta \hat{r}^{\prime \prime}-3 \Delta \hat{r}-2 \Delta \hat{\theta}^{\prime} & =\epsilon\left(-3 \Delta \hat{r}^{2}+2 \Delta \hat{r} \Delta \hat{\theta}^{\prime}-\Delta \hat{\phi}^{2}+\Delta \hat{\theta}^{\prime 2}+\Delta \hat{\phi}^{2}\right) \\
\Delta \hat{\theta}^{\prime \prime}+2 \Delta \hat{r}^{\prime} & =\epsilon\left(2 \Delta \hat{r} \Delta \hat{r}^{\prime}+2 \Delta \hat{\phi} \Delta \hat{\phi}^{\prime}-2 \Delta \hat{\theta}^{\prime} \Delta \hat{r}^{\prime}\right) \\
\Delta \hat{\phi}^{\prime \prime}+\Delta \hat{\phi} & =\epsilon\left(-2 \Delta \hat{\theta}^{\prime} \Delta \hat{\phi}-2 \Delta \hat{\phi}^{\prime} \Delta \hat{r}^{\prime}\right)
\end{aligned}
$$

A place keeping parameter, $\epsilon$, as described in chapter (2), has been introduced into the problem since there is no small parameter. In order for a truncated Taylor expansion to be valid, the variables $\Delta r, \Delta \theta$ and $\Delta \phi$ should be small themselves. If these variables are of order $\rho<<1$, then the perturbation terms will be of order $\rho^{2}$ since the nonlinearity of this problem is quadratic. Therefore, the perturbation of the linear system is of higher order by itself, validating the use of a place keeping parameter. 
Note that the in-plane motion has re-coupled with the out-of-plane motion by expanding the equations of motion to second order. Even without the fact that these equations represent a better approximation of the dynamics, this re-coupling is a further advantage.

\subsection{The Method of Multiple Scales}

The method of multiple scales seeks an approximate solution of equations $(4.10-4.12)$ by making the expansion

$$
\begin{aligned}
& \Delta \hat{r}(\tau)=\Delta \hat{r}_{0}\left(T_{0}, T_{1}, T_{2}\right)+\epsilon \Delta \hat{r}_{1}\left(T_{0}, T_{1}, T_{2}\right)+\epsilon^{2} \Delta \hat{r}_{2}\left(T_{0}, T_{1}, T_{2}\right)+\cdots \\
& \Delta \hat{\theta}(\tau)=\Delta \hat{\theta}_{0}\left(T_{0}, T_{1}, T_{2}\right)+\epsilon \Delta \hat{\theta}_{1}\left(T_{0}, T_{1}, T_{2}\right)+\epsilon^{2} \Delta \hat{\theta}_{2}\left(T_{0}, T_{1}, T_{2}\right)+\cdots \\
& \Delta \hat{\phi}(\tau)=\Delta \hat{\phi}_{0}\left(T_{0}, T_{1}, T_{2}\right)+\epsilon \Delta \hat{\phi}_{1}\left(T_{0}, T_{1}, T_{2}\right)+\epsilon^{2} \Delta \hat{\phi}_{2}\left(T_{0}, T_{1}, T_{2}\right)+\cdots
\end{aligned}
$$

where $T_{0}=\tau, T_{1}=\epsilon \tau$ and $T_{2}=\epsilon^{2} \tau$ are the fast, slow and even slower time scales. Substituting (4.13), (4.14) and (4.15) into (4.10), (4.11) and (4.12) and using the the expansion of the differential operator given in (2.38) and (2.39) for order $\epsilon^{0}$ results in

$$
\begin{aligned}
\frac{\partial^{2} \Delta \hat{r}_{0}}{\partial T_{0}^{2}}-3 \Delta \hat{r}_{0}-2 \frac{\partial \Delta \hat{\theta}_{0}}{\partial T_{0}} & =0 \\
\frac{\partial^{2} \Delta \hat{\theta}_{0}}{\partial T_{0}^{2}}+2 \frac{\partial \Delta \hat{r}_{0}}{\partial T_{0}} & =0 \\
\frac{\partial^{2} \Delta \hat{\phi}_{0}}{\partial T_{0}^{2}}+\Delta \hat{\phi}_{0} & =0
\end{aligned}
$$

Grouping terms of order $\epsilon^{1}$ yields

$$
\begin{aligned}
\frac{\partial^{2} \Delta \hat{r}_{1}}{\partial T_{0}^{2}}-3 \Delta \hat{r}_{1}-2 \frac{\partial \Delta \hat{\theta}_{1}}{\partial T_{0}}= & -2 \frac{\partial^{2} \Delta \hat{r}_{0}}{\partial T_{0} \partial T_{1}}+2 \frac{\partial \Delta \hat{\theta}_{0}}{\partial T_{1}}-3 \Delta \hat{r}_{0}^{2}+2 \Delta \hat{r}_{0} \frac{\partial \Delta \hat{\theta}_{0}}{\partial T_{0}}-\Delta \hat{\phi}_{0}^{2}+ \\
& +\left(\frac{\partial \Delta \hat{\theta}_{0}}{\partial T_{0}}\right)^{2}+\left(\frac{\partial \Delta \hat{\phi}_{0}}{\partial T_{0}}\right)^{2} \\
\frac{\partial^{2} \Delta \hat{\theta}_{1}}{\partial T_{0}^{2}}+2 \frac{\partial \Delta \hat{r}_{1}}{\partial T_{0}}= & -2 \frac{\partial^{2} \Delta \hat{\theta}_{0}}{\partial T_{0} \partial T_{1}}-2 \frac{\partial \Delta \hat{r}_{0}}{\partial T_{1}}+2 \Delta \hat{r}_{0} \frac{\partial \Delta \hat{r}_{0}}{\partial T_{0}}+2 \Delta \hat{\phi}_{0} \frac{\partial \Delta \hat{\phi}_{0}}{\partial T_{0}}- \\
& -2 \frac{\partial \Delta \hat{\theta}_{0}}{\partial T_{0}} \frac{\partial \Delta \hat{r}_{0}}{\partial T_{0}} \\
\frac{\partial^{2} \Delta \hat{\phi}_{1}}{\partial T_{0}^{2}}+\Delta \hat{\phi}_{1}= & -2 \frac{\partial^{2} \Delta \hat{\phi}_{0}}{\partial T_{0} \partial T_{1}}-2 \Delta \hat{\phi}_{0} \frac{\partial \Delta \hat{\theta}_{0}}{\partial T_{0}}-2 \frac{\partial \Delta \hat{\phi}_{0}}{\partial T_{0}} \frac{\partial \hat{r}_{0}}{\partial T_{0}}
\end{aligned}
$$

Finally, grouping terms of order $\epsilon^{2}$ leads to

$$
\frac{\partial^{2} \Delta \hat{r}_{2}}{\partial T_{0}^{2}}-3 \Delta \hat{r}_{2}-2 \frac{\partial \Delta \hat{\theta}_{2}}{\partial T_{0}}=-2 \frac{\partial^{2} \Delta \hat{r}_{1}}{\partial T_{0} \partial T_{1}}-2 \frac{\partial^{2} \Delta \hat{r}_{0}}{\partial T_{0} \partial T_{2}}-\frac{\partial^{2} \Delta \hat{r}_{0}}{\partial T_{1}^{2}}+2 \frac{\partial \Delta \hat{\theta}_{0}}{\partial T_{2}}+2 \frac{\partial \Delta \hat{\theta}_{1}}{\partial T_{1}}-
$$




$$
\begin{aligned}
& -6 \Delta \hat{r}_{0} \Delta \hat{r}_{1}+2 \Delta \hat{r}_{1} \frac{\partial \Delta \hat{\theta}_{0}}{\partial T_{0}}+2 \Delta \hat{r}_{0} \frac{\partial \Delta \hat{\theta}_{0}}{\partial T_{1}}+2 \Delta \hat{r}_{0} \frac{\partial \Delta \hat{\theta}_{1}}{\partial T_{0}}- \\
& -2 \Delta \hat{\phi}_{0} \Delta \hat{\phi}_{1}+2 \frac{\partial \Delta \hat{\theta}_{0}}{\partial T_{0}} \frac{\partial \Delta \hat{\theta}_{0}}{\partial T_{1}}+2 \frac{\partial \Delta \hat{\theta}_{0}}{\partial T_{0}} \frac{\partial \Delta \hat{\theta}_{1}}{\partial T_{0}}+ \\
& +2 \frac{\partial \Delta \hat{\phi}_{0}}{\partial T_{0}} \frac{\partial \Delta \hat{\phi}_{0}}{\partial T_{1}}+2 \frac{\partial \Delta \hat{\phi}_{0}}{\partial T_{0}} \frac{\partial \Delta \hat{\phi}_{1}}{\partial T_{0}} \\
& \frac{\partial^{2} \Delta \hat{\theta}_{2}}{\partial T_{0}^{2}}+2 \frac{\partial \Delta \hat{r}_{2}}{\partial T_{0}}=-2 \frac{\partial^{2} \Delta \hat{\theta}_{1}}{\partial T_{0} \partial T_{1}}-2 \frac{\partial^{2} \Delta \hat{\theta}_{0}}{\partial T_{0} \partial T_{2}}-\frac{\partial^{2} \Delta \hat{\theta}_{0}}{\partial T_{1}^{2}}-2 \frac{\partial \Delta \hat{r}_{0}}{\partial T_{2}}-2 \frac{\partial \Delta \hat{r}_{1}}{\partial T_{1}}+ \\
& +2 \Delta \hat{r}_{0} \frac{\partial \Delta \hat{r}_{1}}{\partial T_{0}}+2 \Delta \hat{r}_{0} \frac{\partial \Delta \hat{r}_{0}}{\partial T_{1}}+2 \Delta \hat{r}_{1} \frac{\partial \Delta \hat{r}_{0}}{\partial T_{0}}+2 \Delta \hat{\phi}_{0} \frac{\partial \Delta \hat{\phi}_{1}}{\partial T_{0}}+ \\
& +2 \Delta \hat{\phi}_{0} \frac{\partial \Delta \hat{\phi}_{0}}{\partial T_{1}}+2 \Delta \hat{\phi}_{1} \frac{\partial \Delta \hat{\phi}_{0}}{\partial T_{0}}-2 \frac{\partial \Delta \hat{\theta}_{0}}{\partial T_{0}} \frac{\partial \Delta \hat{r}_{1}}{\partial T_{0}}- \\
& -2 \frac{\partial \Delta \hat{\theta}_{0}}{\partial T_{0}} \frac{\partial \Delta \hat{r}_{0}}{\partial T_{1}}-2 \frac{\partial \Delta \hat{\theta}_{1}}{\partial T_{0}} \frac{\partial \Delta \hat{r}_{0}}{\partial T_{0}}-2 \frac{\partial \Delta \hat{\theta}_{0}}{\partial T_{1}} \frac{\partial \Delta \hat{r}_{0}}{\partial T_{0}} \\
& \frac{\partial^{2} \Delta \hat{\phi}_{2}}{\partial T_{0}^{2}}+\Delta \hat{\phi}_{2}=-2 \frac{\partial^{2} \Delta \hat{\phi}_{1}}{\partial T_{0} \partial T_{1}}-2 \frac{\partial^{2} \Delta \hat{\phi}_{0}}{\partial T_{0} \partial T_{2}}-\frac{\partial^{2} \Delta \hat{\phi}_{0}}{\partial T_{1}^{2}}-2 \Delta \hat{\phi}_{1} \frac{\partial \Delta \hat{\theta}_{0}}{\partial T_{0}}- \\
& -2 \Delta \hat{\phi}_{0} \frac{\partial \Delta \hat{\theta}_{1}}{\partial T_{0}}-2 \Delta \hat{\phi}_{0} \frac{\partial \Delta \hat{\theta}_{0}}{\partial T_{1}}-2 \frac{\partial \Delta \hat{\phi}_{0}}{\partial T_{0}} \frac{\partial \Delta \hat{r}_{0}}{\partial T_{1}}- \\
& -2 \frac{\partial \Delta \hat{\phi}_{0}}{\partial T_{0}} \frac{\partial \Delta \hat{r}_{1}}{\partial T_{0}}-2 \frac{\partial \Delta \hat{\phi}_{0}}{\partial T_{1}} \frac{\partial \Delta \hat{r}_{0}}{\partial T_{0}}-2 \frac{\partial \Delta \hat{\phi}_{1}}{\partial T_{0}} \frac{\partial \Delta \hat{r}_{0}}{\partial T_{0}}
\end{aligned}
$$

Equations (4.16), (4.17) and (4.18) are the equivalent to equations (3.59), (3.60) and (3.61)except that they are expressed as a system of partial differential equations with respect to the independent variable $T_{0}$ rather than ordinary differential equations. This being the case, the solution of (4.16), (4.17) and (4.18) takes the same form as (3.104), (3.105) and (3.106), but now, $A, B$, $C$ and $D$ are functions of the slow time scales $T_{1}$ and $T_{2}$. The solution is then

$$
\begin{aligned}
& \Delta \hat{r}_{0}=A\left(T_{1}, T_{2}\right) \mathrm{e}^{i T_{0}}+\bar{A}\left(T_{1}, T_{2}\right) \mathrm{e}^{-i T_{0}}+B\left(T_{1}, T_{2}\right) \\
& \Delta \hat{\theta}_{0}=2 i A\left(T_{1}, T_{2}\right) \mathrm{e}^{i T_{0}}-2 i \bar{A}\left(T_{1}, T_{2}\right) \mathrm{e}^{-i T_{0}}-\frac{3}{2} B\left(T_{1}, T_{2}\right) T_{0}+C\left(T_{1}, T_{2}\right) \\
& \Delta \hat{\phi}_{0}=D\left(T_{1}, T_{2}\right) \mathrm{e}^{i T_{0}}+\bar{D}\left(T_{1}, T_{2}\right) \mathrm{e}^{-i T_{0}}
\end{aligned}
$$

These equations will be used as the starting point in the process of assembling the total solution (4.13), (4.14) and (4.15).

Note that six more constants of integration will be required to solve the partial differential equations at each order of $\epsilon$. The typical approach is to set these constants to zero. This approach leads to a re-definition of the constants of integration between the linear case and the nonlinear case. In order to more readily compare the linear solution to the nonlinear solution, it is desirable that the definition of the constants remains the same. Therefore, the initial conditions of the problem may be expressed as

$$
\Delta \hat{r}(0)=\Delta \hat{r}_{0}(0)+\epsilon \Delta \hat{r}_{1}(0)+\epsilon^{2} \Delta \hat{r}_{2}(0)
$$




$$
\begin{aligned}
\Delta \hat{\theta}(0)= & \Delta \hat{\theta}_{0}(0)+\epsilon \Delta \hat{\theta}_{1}(0)+\epsilon^{2} \Delta \hat{\theta}_{2}(0) \\
\Delta \hat{\phi}(0)= & \Delta \hat{\phi}_{0}(0)+\epsilon \Delta \hat{\phi}_{1}(0)+\epsilon^{2} \Delta \hat{\phi}_{2}(0) \\
\Delta \hat{v}_{r}(0)= & \frac{\partial \Delta \hat{r}_{0}}{\partial T_{0}}(0)+\epsilon\left(\frac{\partial \Delta \hat{r}_{0}}{\partial T_{1}}(0)+\frac{\partial \Delta \hat{r}_{1}}{\partial T_{0}}(0)\right)+ \\
& +\epsilon^{2}\left(\frac{\partial \Delta \hat{r}_{0}}{\partial T_{2}}(0)+\frac{\partial \Delta \hat{r}_{1}}{\partial T_{1}}(0)+\frac{\partial \Delta \hat{r}_{2}}{\partial T_{0}}(0)\right) \\
\Delta \hat{\omega}_{\theta}(0)= & \frac{\partial \Delta \hat{\theta}_{0}}{\partial T_{0}}(0)+\epsilon\left(\frac{\partial \Delta \hat{\theta}_{0}}{\partial T_{1}}(0)+\frac{\partial \Delta \hat{\theta}_{1}}{\partial T_{0}}(0)\right)+ \\
& +\epsilon^{2}\left(\frac{\partial \Delta \hat{\theta}_{0}}{\partial T_{2}}(0)+\frac{\partial \Delta \hat{\theta}_{1}}{\partial T_{1}}(0)+\frac{\partial \Delta \hat{\theta}_{2}}{\partial T_{0}}(0)\right) \\
\Delta \hat{\omega}_{\phi}(0)= & \frac{\partial \Delta \hat{\phi}_{0}}{\partial T_{0}}(0)+\epsilon\left(\frac{\partial \Delta \hat{\phi}_{0}}{\partial T_{1}}(0)+\frac{\partial \Delta \hat{\phi}_{1}}{\partial T_{0}}(0)\right)+ \\
& +\epsilon^{2}\left(\frac{\partial \Delta \hat{\phi}_{0}}{\partial T_{2}}(0)+\frac{\partial \Delta \hat{\phi}_{1}}{\partial T_{1}}+\frac{\partial \Delta \hat{\phi}_{2}}{\partial T_{0}}(0)\right)
\end{aligned}
$$

In order to enforce the initial conditions of the problem on the linear portion of the solution alone, it must be true that

$$
\begin{aligned}
\Delta \hat{r}_{1}(0) & =0 \\
\Delta \hat{r}_{2}(0) & =0 \\
\Delta \hat{\theta}_{1}(0) & =0 \\
\Delta \hat{\theta}_{2}(0) & =0 \\
\Delta \hat{\phi}_{1}(0) & =0 \\
\Delta \hat{\phi}_{2}(0) & =0 \\
\frac{\partial \Delta \hat{r}_{1}}{\partial T_{0}}(0) & =-\frac{\partial \Delta \hat{r}_{0}}{\partial T_{1}}(0) \\
\frac{\partial \Delta \hat{r}_{2}}{\partial T_{0}}(0) & =-\frac{\partial \Delta \hat{r}_{0}}{\partial T_{2}}(0)-\frac{\partial \Delta \hat{r}_{1}}{\partial T_{1}}(0) \\
\frac{\partial \Delta \hat{\theta}_{1}}{\partial T_{0}}(0) & =-\frac{\partial \Delta \hat{\theta}_{0}}{\partial T_{1}}(0) \\
\frac{\partial \Delta \hat{\theta}_{2}}{\partial T_{0}}(0) & =-\frac{\partial \Delta \hat{\theta}_{0}}{\partial T_{2}}(0)-\frac{\partial \Delta \hat{\theta}_{1}}{\partial T_{1}}(0) \\
\frac{\partial \Delta \hat{\phi}_{1}}{\partial T_{0}}(0) & =-\frac{\partial \Delta \hat{\phi}_{0}}{\partial T_{1}}(0) \\
\frac{\partial \Delta \hat{\phi}_{2}}{\partial T_{0}}(0) & =-\frac{\partial \Delta \hat{\phi}_{0}}{\partial T_{2}}(0)-\frac{\partial \Delta \hat{\phi}_{1}}{\partial T_{1}}(0)
\end{aligned}
$$

The nonzero initial conditions on the first and second order partial differential equations will 
be defined as

$$
\begin{aligned}
& \frac{\partial \Delta \hat{r}_{1}}{\partial T_{0}}(0)=\Delta \hat{v}_{r_{10}} \\
& \frac{\partial \Delta \hat{r}_{2}}{\partial T_{0}}(0)=\Delta \hat{v}_{r_{20}} \\
& \frac{\partial \Delta \hat{\theta}_{1}}{\partial T_{0}}(0)=\Delta \hat{\omega}_{\theta_{10}} \\
& \frac{\partial \Delta \hat{\theta}_{2}}{\partial T_{0}}(0)=\Delta \hat{\omega}_{\theta_{20}} \\
& \frac{\partial \Delta \hat{\phi}_{1}}{\partial T_{0}}(0)=\Delta \hat{\omega}_{\phi_{10}} \\
& \frac{\partial \Delta \hat{\phi}_{2}}{\partial T_{0}}(0)=\Delta \hat{\omega}_{\phi_{20}}
\end{aligned}
$$

\subsubsection{First Order Solution}

Evaluating the equation (4.19), (4.20) and (4.21) using the solution (4.25), (4.26) and (4.27) is an algebraically complicated task, and is given in appendix (B). The first order (in terms of $\epsilon$ ) system of partial differential equations becomes

$$
\begin{aligned}
\frac{\partial^{2} \Delta \hat{r}_{1}}{\partial T_{0}^{2}}-3 \Delta \hat{r}_{1}-2 \frac{\partial \Delta \hat{\theta}_{1}}{\partial T_{0}}= & \left(2 i \frac{\partial A}{\partial T_{1}}-7 A B\right) \mathrm{e}^{i T_{0}}+\left(-2 i \frac{\partial \bar{A}}{\partial T_{1}}-7 \bar{A} B\right) \mathrm{e}^{-i T_{0}}+ \\
& +\left(-3 A^{2}-2 D^{2}\right) \mathrm{e}^{2 i T_{0}}+\left(-3 \bar{A}^{2}-2 \bar{D}^{2}\right) \mathrm{e}^{-2 i T_{0}}+ \\
& +2 \frac{\partial C}{\partial T_{1}}-\frac{15 B^{2}}{4}-6 A \bar{A}-3 \frac{\partial B}{\partial T_{1}} T_{0} \\
\frac{\partial^{2} \Delta \hat{\theta}_{1}}{\partial T_{0}^{2}}+2 \frac{\partial \Delta \hat{r}_{1}}{\partial T_{0}}= & \left(2 \frac{\partial A}{\partial T_{1}}+5 i A B\right) \mathrm{e}^{i T_{0}}+\left(2 \frac{\partial \bar{A}}{\partial T_{1}}-5 i \bar{A} B\right) \mathrm{e}^{-i T_{0}}+ \\
& +\left(6 i A^{2}+2 i D^{2}\right) \mathrm{e}^{2 i T_{0}}+\left(-6 i \bar{A}^{2}-2 i \bar{D}^{2}\right) \mathrm{e}^{-2 i T_{0}}- \\
& -2 \frac{\partial B}{\partial T_{1}} \\
\frac{\partial^{2} \Delta \hat{\phi}_{1}}{\partial T_{0}^{2}}+\Delta \hat{\phi}_{1}= & \left(-2 i \frac{\partial D}{\partial T_{1}}+3 D B\right) \mathrm{e}^{i T_{0}}+\left(2 i \frac{\partial \bar{D}}{\partial T_{1}}+3 \bar{D} B\right) \mathrm{e}^{-i T_{0}}+ \\
& +6 A D \mathrm{e}^{2 i T_{0}}+6 \bar{A} \bar{D} \mathrm{e}^{-2 i T_{0}}+2 \bar{A} D+2 A \bar{D}
\end{aligned}
$$

At this stage it is convenient to introduce several intermediate variables (all functions of the 
slow time scales, $T_{1}$ and $\left.T_{2}\right), \gamma_{r_{1}}, \gamma_{\theta_{r_{1}}}, \gamma_{\phi_{1}} \eta_{r_{1}}, \eta_{\theta_{1}}, \eta_{\phi_{1}}, \sigma_{r_{1}}, \sigma_{\theta_{1}}, \sigma_{\phi_{1}}$ and $\lambda_{r_{1}}$ defined such that

$$
\begin{aligned}
& \gamma_{r_{1}}=2 i \frac{\partial A}{\partial T_{1}}-7 A B \\
& \gamma_{\theta_{1}}=2 \frac{\partial A}{\partial T_{1}}+5 i A B \\
& \gamma_{\phi_{1}}=3 D B-2 i \frac{\partial D}{\partial T_{1}} \\
& \eta_{r_{1}}=-3 A^{2}-2 D^{2} \\
& \eta_{\theta_{1}}=6 i A^{2}+2 i D^{2} \\
& \eta_{\phi_{1}}=6 A D \\
& \sigma_{r_{1}}=2 \frac{\partial C}{\partial T_{1}}-\frac{15 B^{2}}{4}-6 A \bar{A} \\
& \sigma_{\theta_{1}}=-2 \frac{\partial B}{\partial T_{1}} \\
& \sigma_{\phi_{1}}=2 \bar{A} D+2 A \bar{D} \\
& \lambda_{r_{1}}=-3 \frac{\partial B}{\partial T_{1}}
\end{aligned}
$$

Using these definitions, equations (4.52), (4.53) and (4.54) become

$$
\begin{gathered}
\frac{\partial^{2} \Delta \hat{r}_{1}}{\partial T_{0}^{2}}-3 \Delta \hat{r}_{1}-2 \frac{\partial \Delta \hat{\theta}_{1}}{\partial T_{0}}=\gamma_{r_{1}} \mathrm{e}^{i T_{0}}+\bar{\gamma}_{r_{1}} \mathrm{e}^{-i T_{0}}+\eta_{r_{1}} \mathrm{e}^{2 i T_{0}}+\bar{\eta}_{r_{1}} \mathrm{e}^{-2 i T_{0}}+\sigma_{r_{1}}+\lambda_{r_{1}} T_{0} \\
\frac{\partial^{2} \Delta \hat{\theta}_{1}}{\partial T_{0}^{2}}+2 \frac{\partial \Delta \hat{r}_{1}}{\partial T_{0}}=\gamma_{\theta_{1}} \mathrm{e}^{i T_{0}}+\bar{\gamma}_{\theta_{1}} \mathrm{e}^{-i T_{0}}+\eta_{\theta_{1}} \mathrm{e}^{2 i T_{0}}+\bar{\eta}_{\theta_{1}} \mathrm{e}^{-2 i T_{0}}+\sigma_{\theta_{1}} \\
\frac{\partial^{2} \Delta \hat{\phi}_{1}}{\partial T_{0}^{2}}+\Delta \hat{\phi}_{1}=\gamma_{\phi_{1}} \mathrm{e}^{i T_{0}}+\bar{\gamma}_{\phi_{1}} \mathrm{e}^{-i T_{0}}+\eta_{\phi_{1}} \mathrm{e}^{2 i T_{0}}+\bar{\eta}_{\phi_{1}} \mathrm{e}^{-2 i T_{0}}+\sigma_{\phi_{1}}
\end{gathered}
$$

Again, note that these equations are the same linear system of partial differential equations found in (4.16), (4.17) and (4.18) but now there appears a forcing function for each equation. The out-of-plane equation, (4.67), has again de-coupled from the in-plane equations, (4.65) and (4.66). Furthermore, since these are partial differential equations in terms of $T_{0}$ only, any function that varies with respect to $T_{1}$ or $T_{2}$ is fixed. This condition allows for the system to be solved by means of Laplace transforms as in chapter (3). First, the Laplace transform of the 
in-plane equation (using the initial conditions previously discussed) is

$$
\left[\begin{array}{cc}
s^{2}-3 & -2 s \\
2 s & s^{2}
\end{array}\right]\left(\begin{array}{c}
\Delta \hat{R}_{1} \\
\Delta \hat{\Theta}_{1}
\end{array}\right)=\left(\begin{array}{c}
\frac{\gamma_{r_{1}}}{s-i}+\frac{\bar{\gamma}_{r_{1}}}{s+i}+\frac{\eta_{r_{1}}}{s-2 i}+\frac{\bar{\eta}_{r_{1}}}{s+2 i}+\frac{\sigma_{r_{1}}}{s}+\frac{\lambda_{r_{1}}}{s^{2}}+\Delta \hat{v}_{r_{10}} \\
\frac{\gamma_{\theta_{1}}}{s-i}+\frac{\bar{\gamma}_{\theta_{1}}}{s+i}+\frac{\eta_{\theta_{1}}}{s-2 i}+\frac{\eta_{\theta_{1}}}{s+2 i}+\frac{\sigma_{\theta_{1}}}{s}+\Delta \hat{\omega}_{\phi_{10}}
\end{array}\right)
$$

Solving for $\Delta \hat{R}_{1}$ and $\Delta \hat{\Theta}_{1}$ results in

$$
\left(\begin{array}{c}
\Delta \hat{R}_{1} \\
\Delta \hat{\Theta}_{1}
\end{array}\right)=\frac{1}{s^{2}\left(s^{2}+1\right)}\left[\begin{array}{cc}
s^{2} & 2 s \\
-2 s & s^{2}-3
\end{array}\right]\left(\begin{array}{c}
\frac{\gamma_{r_{1}}}{s-i}+\frac{\bar{\gamma}_{r_{1}}}{s+i}+\frac{\eta_{r_{1}}}{s-2 i}+\frac{\bar{\eta}_{r_{1}}}{s+2 i}+\frac{\sigma_{r_{1}}}{s}+\frac{\lambda_{r_{1}}}{s^{2}}+\Delta \hat{v}_{r_{10}} \\
\frac{\gamma_{\theta_{1}}}{s-i}+\frac{\bar{\gamma}_{\theta_{1}}}{s+i}+\frac{\eta_{\theta_{1}}}{s-2 i}+\frac{\bar{\eta}_{\theta_{1}}}{s+2 i}+\frac{\sigma_{\theta_{1}}}{s}+\Delta \hat{\omega}_{\theta_{10}}
\end{array}\right)
$$

Or,

$$
\begin{aligned}
\Delta \hat{R}_{1}= & \frac{\gamma_{r_{1}}}{\left(s^{2}+1\right)(s-i)}+\frac{\bar{\gamma}_{r_{1}}}{\left(s^{2}+1\right)(s+i)}+\frac{\eta_{r_{1}}}{\left(s^{2}+1\right)(s-2 i)}+\frac{\bar{\eta}_{r_{1}}}{\left(s^{2}+1\right)(s+2 i)}+ \\
& +\frac{\lambda_{r_{1}}+2 \sigma_{\theta_{1}}}{s^{2}\left(s^{2}+1\right)}+\frac{\sigma_{r_{1}}}{s\left(s^{2}+1\right)}+\frac{2 \gamma_{\theta_{1}}}{s\left(s^{2}+1\right)(s-i)}+\frac{2 \bar{\gamma}_{\theta_{1}}}{s\left(s^{2}+1\right)(s+i)}+ \\
& +\frac{2 \eta_{\theta_{1}}}{s\left(s^{2}+1\right)(s-2 i)}+\frac{2 \bar{\eta}_{\theta_{1}}}{s\left(s^{2}+1\right)(s+2 i)}+\frac{\Delta \hat{v}_{r_{10}}}{s^{2}+1}+\frac{2 \Delta \hat{\omega}_{\theta_{10}}}{s\left(s^{2}+1\right)} \\
\Delta \hat{\Theta}_{1}= & -\frac{2 \gamma_{r_{1}}}{s\left(s^{2}+1\right)(s-i)}-\frac{2 \bar{\gamma}_{r_{1}}}{s\left(s^{2}+1\right)(s+i)}-\frac{2 \eta_{r_{1}}}{s\left(s^{2}+1\right)(s-2 i)}-\frac{2 \bar{\eta}_{r_{1}}}{s\left(s^{2}+1\right)(s+2 i)}- \\
& -\frac{2 \lambda_{r_{1}}}{s^{3}\left(s^{2}+1\right)}-\frac{2 \sigma_{r_{1}}}{s^{2}\left(s^{2}+1\right)}+\frac{\gamma_{\theta_{1}}\left(s^{2}-3\right)}{s^{2}\left(s^{2}+1\right)(s-i)}+\frac{\bar{\gamma}_{\theta_{1}}\left(s^{2}-3\right)}{s^{2}\left(s^{2}+1\right)(s+i)}+ \\
& +\frac{\eta_{\theta_{1}}\left(s^{2}-3\right)}{s^{2}\left(s^{2}+1\right)(s-2 i)}+\frac{\bar{\eta}_{\theta_{1}}\left(s^{2}-3\right)}{s^{2}\left(s^{2}+1\right)(s+2 i)}+\frac{\sigma_{\theta_{1}}\left(s^{2}-3\right)}{s^{3}\left(s^{2}+1\right)}+\frac{\left(s^{2}-3\right) \Delta \hat{\omega}_{\theta_{10}}}{s^{2}\left(s^{2}+1\right)}- \\
& -\frac{2 \Delta \hat{v}_{r_{10}}}{s\left(s^{2}+1\right)}
\end{aligned}
$$

The inverse Laplace transforms are

$$
\begin{aligned}
\mathcal{L}^{-1}\left[\frac{1}{\left(s^{2}+1\right)(s \pm i)}\right] & = \pm\left(\frac{1}{2} i T_{0} \mathrm{e}^{\mp i T_{0}}+\frac{1}{4} \mathrm{e}^{-i T_{0}}-\frac{1}{4} \mathrm{e}^{i T_{0}}\right) \\
\mathcal{L}^{-1}\left[\frac{1}{\left(s^{2}+1\right)(s \pm 2 i)}\right] & =\frac{1}{2} \mathrm{e}^{\mp i T_{0}}-\frac{1}{6} \mathrm{e}^{ \pm i T_{0}}-\frac{1}{3} \mathrm{e}^{\mp 2 i T_{0}} \\
\mathcal{L}^{-1}\left[\frac{1}{s\left(s^{2}+1\right)(s \pm i)}\right] & =-\frac{1}{2} T_{0} \mathrm{e}^{\mp i T_{0}} \mp i\left(1-\frac{3}{4} \mathrm{e}^{\mp i T_{0}}-\frac{1}{4} \mathrm{e}^{ \pm i T_{0}}\right) \\
\mathcal{L}^{-1}\left[\frac{1}{s\left(s^{2}+1\right)(s \pm 2 i)}\right] & = \pm\left(\frac{1}{2} \mathrm{e}^{\mp i T_{0}}+\frac{1}{6} \mathrm{e}^{ \pm i T_{0}}-\frac{1}{6} \mathrm{e}^{\mp 2 i T_{0}}-1\right) \\
\mathcal{L}^{-1}\left[\frac{1}{s^{2}\left(s^{2}+1\right)(s \pm i)}\right] & =1-\frac{5}{4} \mathrm{e}^{\mp i T_{0}}+\frac{1}{4} \mathrm{e}^{ \pm i T_{0}} \mp \frac{1}{2} i T_{0} \mathrm{e}^{\mp i T_{0}} \mp i T_{0} \\
\mathcal{L}^{-1}\left[\frac{1}{s^{2}\left(s^{2}+1\right)(s \pm 2 i)}\right] & =\frac{1}{4}-\frac{1}{2} \mathrm{e}^{\mp i T_{0}}+\frac{1}{6} \mathrm{e}^{ \pm i T_{0}}+\frac{1}{12} \mathrm{e}^{\mp 2 i T_{0}} \mp \frac{1}{2} i T_{0} \\
\mathcal{L}^{-1}\left[\frac{1}{s\left(s^{2}+1\right)}\right] & =1-\frac{1}{2} \mathrm{e}^{i T_{0}}-\frac{1}{2} \mathrm{e}^{-i T_{0}}
\end{aligned}
$$


Chris Karlgaard

$$
\begin{aligned}
& \mathcal{L}^{-1}\left[\frac{1}{s^{2}\left(s^{2}+1\right)}\right]=T_{0}+\frac{1}{2} i \mathrm{e}^{i T_{0}}-\frac{1}{2} i \mathrm{e}^{-i T_{0}} \\
& \mathcal{L}^{-1}\left[\frac{1}{s^{3}\left(s^{2}+1\right)}\right]=\frac{1}{2} T_{0}^{2}+\frac{1}{2} \mathrm{e}^{i T_{0}}+\frac{1}{2} \mathrm{e}^{-i T_{0}}-1
\end{aligned}
$$

Assembling these leads to

$$
\begin{aligned}
& \Delta \hat{r}_{1}=\left(\frac{\gamma_{r_{1}}}{4}+\frac{\eta_{r_{1}}}{2}-\frac{\bar{\gamma}_{r_{1}}}{4}+\frac{i \bar{\gamma}_{\theta_{1}}}{2}-\frac{\bar{\eta}_{r_{1}}}{6}+\frac{i \bar{\eta}_{\theta_{1}}}{3}-\frac{3 i \gamma_{\theta_{1}}}{2}-i \eta_{\theta_{1}}+\frac{i \lambda_{r_{1}}}{2}-\frac{\sigma_{r_{1}}}{2}+i \sigma_{\theta_{1}}-\right. \\
& \left.-\frac{i \Delta \hat{v}_{r_{10}}}{2}-\Delta \hat{\omega}_{\theta_{10}}-\left[\gamma_{\theta_{1}}+\frac{i \gamma_{r_{1}}}{2}\right] T_{0}\right) \mathrm{e}^{i T_{0}}+\left(\frac{\bar{\gamma}_{r_{1}}}{4}+\frac{\bar{\eta}_{r_{1}}}{2}-\frac{\gamma_{r_{1}}}{4}-\frac{i \gamma_{\theta_{1}}}{2}-\frac{\eta_{r_{1}}}{6}-\frac{i \eta_{\theta_{1}}}{3}+\right. \\
& \left.+\frac{3 i \bar{\gamma}_{\theta_{1}}}{2}+i \bar{\eta}_{\theta_{1}}-\frac{i \lambda_{r_{1}}}{2}-\frac{\sigma_{r_{1}}}{2}-i \sigma_{\theta_{1}}+\frac{i \Delta \hat{v}_{r_{10}}}{2}-\Delta \hat{\omega}_{\theta_{10}}-\left[\bar{\gamma}_{\theta_{1}}-\frac{i \bar{\gamma}_{r_{1}}}{2}\right] T_{0}\right) \mathrm{e}^{-i T_{0}}+ \\
& +\left(\frac{i \eta_{\theta_{1}}}{3}-\frac{\eta_{r_{1}}}{3}\right) \mathrm{e}^{2 i T_{0}}+\left(-\frac{i \bar{\eta}_{\theta_{1}}}{3}-\frac{\bar{\eta}_{r_{1}}}{3}\right) \mathrm{e}^{-2 i T_{0}}+\left(\lambda_{r_{1}}+2 \sigma_{\theta_{1}}\right) T_{0}+2 i \gamma_{\theta_{1}}-2 i \bar{\gamma}_{\theta_{1}}+ \\
& +i \eta_{\theta_{1}}-i \bar{\eta}_{\theta_{1}}+\sigma_{r_{1}}+2 \Delta \hat{\omega}_{\theta_{10}} \\
& \Delta \hat{\theta}_{1}=\left(4 \gamma_{\theta_{1}}+i \eta_{r_{1}}+2 \eta_{\theta_{1}}-\lambda_{r_{1}}-i \sigma_{r_{1}}-2 \sigma_{\theta_{1}}-\frac{i \bar{\gamma}_{r_{1}}}{2}-\bar{\gamma}_{\theta_{1}}-\frac{i \bar{\eta}_{r_{1}}}{3}-\frac{2 \bar{\eta}_{\theta_{1}}}{3}+\frac{3 i \gamma_{r_{1}}}{2}+\right. \\
& \left.+\Delta \hat{v}_{r_{10}}-2 i \Delta \hat{\omega}_{\theta_{10}}+\left[\gamma_{r_{1}}-2 i \gamma_{\theta_{1}}\right] T_{0}\right) \mathrm{e}^{i T_{0}}+\left(4 \bar{\gamma}_{\theta_{1}}-i \bar{\eta}_{r_{1}}+2 \bar{\eta}_{\theta_{1}}-\lambda_{r_{1}}+i \sigma_{r_{1}}-\right. \\
& \left.-2 \sigma_{\theta_{1}}+\frac{i \gamma_{r_{1}}}{2}-\gamma_{\theta_{1}}+\frac{i \eta_{r_{1}}}{3}-\frac{2 \eta_{\theta_{1}}}{3}-\frac{3 i \bar{\gamma}_{r_{1}}}{2}+\Delta \hat{v}_{r_{10}}+2 i \Delta \hat{\omega}_{\theta_{10}}+\left[\bar{\gamma}_{r_{1}}+2 i \bar{\gamma}_{\theta_{1}}\right] T_{0}\right) \mathrm{e}^{-i T_{0}}+ \\
& +\left(-\frac{i \eta_{r_{1}}}{3}-\frac{7 \eta_{\theta_{1}}}{12}\right) \mathrm{e}^{2 i T_{0}}+\left(\frac{i \bar{\eta}_{r_{1}}}{3}-\frac{7 \bar{\eta}_{\theta_{1}}}{12}\right) \mathrm{e}^{-2 i T_{0}}+\left(3 i \bar{\gamma}_{\theta_{1}}-3 i \gamma_{\theta_{1}}+\frac{3 i \bar{\eta}_{\theta_{1}}}{2}-\frac{3 i \eta_{\theta_{1}}}{2}-\right. \\
& \left.-2 \sigma_{r_{1}}-3 \Delta \hat{\omega}_{\theta_{10}}\right) T_{0}-\left(\lambda_{r_{1}}+\frac{3 \sigma_{\theta_{1}}}{2}\right) T_{0}^{2}+2 i \bar{\gamma}_{r_{1}}-2 i \gamma_{r_{1}}-3 \bar{\gamma}_{\theta_{1}}-3 \gamma_{\theta_{1}}+i \bar{\eta}_{r_{1}}- \\
& -i \eta_{r_{1}}-\frac{3 \bar{\eta}_{\theta_{1}}}{4}-\frac{3 \eta_{\theta_{1}}}{4}+2 \lambda_{r_{1}}+4 \sigma_{\theta_{1}}-2 \Delta \hat{v}_{r_{10}}
\end{aligned}
$$

Elimination of secular terms requires that

$$
\begin{gathered}
\lambda_{r_{1}}+2 \sigma_{\theta_{1}}=0 \\
\lambda_{r_{1}}+\frac{3 \sigma_{\theta_{1}}}{2}=0 \\
\gamma_{r_{1}}-2 i \gamma_{\theta_{1}}=0
\end{gathered}
$$

and enforcing the initial conditions of the problem gives,

$$
3 i \bar{\gamma}_{\theta_{1}}-3 i \gamma_{\theta_{1}}+\frac{3 i \bar{\eta}_{\theta_{1}}}{2}-\frac{3 i \eta_{\theta_{1}}}{2}-2 \sigma_{r_{1}}-3 \Delta \hat{\omega}_{\theta_{10}}=0
$$

Note that equation (4.85) appears in several locations in the solution, although in an algebraically different form which therefore contains no new information. Equations (4.83) and (4.84) imply that

$$
\lambda_{r_{1}}=\sigma_{\theta_{1}}=0
$$


which leads to

$$
\frac{\partial B}{\partial T_{1}}=0
$$

Expanding equation (4.85),

$$
\begin{aligned}
2 i \frac{\partial A}{\partial T_{1}}-7 A B & =4 i \frac{\partial A}{\partial T_{1}}-10 A B \\
2 i \frac{\partial A}{\partial T_{1}} & =3 A B
\end{aligned}
$$

which results in

$$
\frac{\partial A}{\partial T_{1}}=-\frac{3}{2} i A B
$$

Equation (4.86) leads to

$$
\begin{aligned}
\sigma_{r_{1}} & =\frac{3}{2} i \bar{\gamma}_{\theta_{1}}-\frac{3}{2} i \gamma_{\theta_{1}}+\frac{3}{4} i \bar{\eta}_{\theta_{1}}-\frac{3}{4} i \eta_{\theta_{1}}-\frac{3}{4} \Delta \hat{\omega}_{\theta_{10}} \\
2 \frac{\partial C}{\partial T_{1}}-\frac{15}{4} B^{2}-6 A \bar{A} & =\frac{3}{2}\left(D^{2}+\bar{D}^{2}\right)+\frac{9}{2}\left(A^{2}+\bar{A}^{2}\right)+3(A B+\bar{A} B)-\frac{3}{2} \Delta \hat{\omega}_{\theta_{10}}
\end{aligned}
$$

so that

$$
\frac{\partial C}{\partial T_{1}}=\frac{15}{8} B^{2}+3 A \bar{A}+\frac{3}{4}\left(D^{2}+\bar{D}^{2}\right)+\frac{9}{4}\left(A^{2}+\bar{A}^{2}\right)+\frac{3}{2}(A B+\bar{A} B)-\frac{3}{4} \Delta \hat{\omega}_{\theta_{10}}
$$

Substituting equations (4.88), (4.91), (4.94) into (4.81) and (4.82) gives the result

$$
\begin{aligned}
\Delta \hat{r}_{1}= & \left(\frac{9}{4} A^{2}+\frac{1}{2} A B+\frac{1}{4} D^{2}+\frac{1}{2} \bar{A} B+\frac{1}{4} \bar{A}^{2}+\frac{1}{4} \bar{D}^{2}-\frac{i \Delta \hat{v}_{r_{10}}}{2}-\frac{\Delta \hat{\omega}_{\theta_{10}}}{4}\right) \mathrm{e}^{i T_{0}}+ \\
& +\left(\frac{9}{4} \bar{A}^{2}+\frac{1}{2} \bar{A} B+\frac{1}{4} \bar{D}^{2}+\frac{1}{2} A B+\frac{1}{4} A^{2}+\frac{1}{4} D^{2}+\frac{i \Delta \hat{v}_{r_{10}}}{2}-\frac{\Delta \hat{\omega}_{\theta_{10}}}{4}\right) \mathrm{e}^{-i T_{0}}- \\
& -A^{2} \mathrm{e}^{2 i T_{0}}-\bar{A}^{2} \mathrm{e}^{-2 i T_{0}}-\frac{3}{2} A^{2}-\frac{3}{2} \bar{A}^{2}-A B-\bar{A} B-\frac{1}{2} D^{2}-\frac{1}{2} \bar{D}^{2}+\frac{\Delta \hat{\omega}_{\theta_{10}}}{2} \\
\Delta \hat{\theta}_{1}= & \left(\frac{9}{2} A^{2}-A B+\frac{1}{2} D^{2}+\bar{A} B+\frac{1}{2} \bar{A}^{2}+\frac{1}{2} \bar{D}^{2}-i \Delta \hat{v}_{r_{10}}-\frac{\Delta \hat{\omega}_{\theta_{10}}}{2}\right) i \mathrm{e}^{i T_{0}}+ \\
& +\left(-\frac{9 \bar{A}^{2}}{2}+\bar{A} B-\frac{1}{2} \bar{D}^{2}-A B-\frac{1}{2} A^{2}-\frac{1}{2} D^{2}-i \Delta \hat{v}_{r_{10}}+\frac{\Delta \hat{\omega}_{\theta_{10}}}{2}\right) i \mathrm{e}^{-i T_{0}}+ \\
& +\left(-\frac{5}{2} A^{2}-\frac{1}{2} D^{2}\right) i \mathrm{e}^{2 i T_{0}}+\left(\frac{5}{2} \bar{A}^{2}+\frac{1}{2} \bar{D}^{2}\right) i \mathrm{e}^{-2 i T_{0}}+ \\
& +\left(-\frac{3}{2} A^{2}+\frac{3}{2} \bar{A}^{2}+2 A B-2 \bar{A} B+\frac{1}{2} D^{2}-\frac{1}{2} \bar{D}^{2}+2 i \Delta \hat{v}_{r_{10}}\right) i
\end{aligned}
$$

Equations (4.95) and (4.96) are the solution of equations (4.52) and (4.53) that have been made free of secular terms by determining conditions on the slowly varying parameters given in 
equations (4.88), (4.91) and (4.94). Next, the Laplace transform of the out-of-plane equation, (4.54), is

$$
\begin{aligned}
\Delta \hat{\Phi}_{1}= & \frac{\gamma_{\phi_{1}}}{\left(s^{2}+1\right)(s-i)}+\frac{\bar{\gamma}_{\phi_{1}}}{\left(s^{2}+1\right)(s+i)}+\frac{\eta_{\phi_{1}}}{\left(s^{2}+1\right)(s-2 i)}+\frac{\bar{\eta}_{\phi_{1}}}{\left(s^{2}+1\right)(s+2 i)}+ \\
& +\frac{\sigma_{\phi_{1}}}{s\left(s^{2}+1\right)}+\frac{\Delta \hat{\omega}_{\phi_{10}}}{s^{2}+1}
\end{aligned}
$$

which has the inverse transformation

$$
\begin{aligned}
\Delta \hat{\phi}_{1}= & \left(\frac{\gamma_{\phi_{1}}}{4}+\frac{\eta_{\phi_{1}}}{2}-\frac{\bar{\gamma}_{\phi_{1}}}{4}-\frac{\bar{\eta}_{\phi_{1}}}{6}-\frac{\sigma_{\phi_{1}}}{2}-\frac{i \Delta \hat{\omega}_{\phi_{10}}}{2}-\frac{i \gamma_{\phi_{1}}}{2} T_{0}\right) \mathrm{e}^{i T_{0}}+ \\
& +\left(\frac{\bar{\gamma}_{\phi_{1}}}{4}+\frac{\bar{\eta}_{\phi_{1}}}{2}-\frac{\gamma_{\phi_{1}}}{4}-\frac{\eta_{\phi_{1}}}{6}-\frac{\sigma_{\phi_{1}}}{2}+\frac{i \Delta \hat{\omega}_{\phi_{10}}}{2}+\frac{i \bar{\gamma}_{\phi_{1}}}{2} T_{0}\right) \mathrm{e}^{-i T_{0}}- \\
& -\frac{\eta_{\phi_{1}}}{3} \mathrm{e}^{2 i T_{0}}-\frac{\bar{\eta}_{\phi_{1}}}{3} \mathrm{e}^{-2 i T_{0}}+\sigma_{\phi_{1}}
\end{aligned}
$$

Eliminating secular terms requires that

$$
\gamma_{\phi_{1}}=0
$$

or, that

$$
\frac{\partial D}{\partial T_{1}}=-\frac{3}{2} i D B
$$

The solution of the out-of-plane equation is

$$
\begin{aligned}
\Delta \hat{\phi}_{1}= & \left(3 A D-\bar{A} D-A \bar{D}-\bar{A} \bar{D}-\frac{i \Delta \hat{\omega}_{\phi_{10}}}{2}\right) \mathrm{e}^{i T_{0}}+ \\
& +\left(3 \bar{A} \bar{D}-A \bar{D}-\bar{A} D-A D+\frac{i \Delta \hat{\omega}_{\phi_{10}}}{2}\right) \mathrm{e}^{-i T_{0}}- \\
& -2 A D \mathrm{e}^{2 i T_{0}}-2 \bar{A} \bar{D} \mathrm{e}^{-2 i T_{0}}+2 A \bar{D}+2 \bar{A} D
\end{aligned}
$$

This section has produced equations for the variables $\Delta \hat{r}_{1}, \Delta \hat{\theta}_{1}$ and $\Delta \hat{\phi}_{1}$ given by (4.95), (4.96) and (4.101), respectively. These equations are the first term in the correction to the linear approximation. Partial differential equations have been found to describe the behavior of $A, B$, $C$ and $D$ in terms of the slow variable, $T_{1}$, in such a way that the solution is free of spurious secular terms. Next, these solutions will be used to construct the system of partial differential equations that will give the next term in the correction series (4.13), (4.14) and (4.15), and the goal is to find more conditions on $A, B, C$ and $D$ in terms of $T_{2}$ in such a way that $\Delta \hat{r}_{2}, \Delta \hat{\theta}_{2}$ and $\Delta \hat{\phi}_{2}$ will also be free of secular terms. 


\subsubsection{Second Order Solution}

The process of evaluating the equations (4.22), (4.23) and (4.24) using the solutions (4.25), (4.26), (4.27), (4.95), (4.96) and (4.101) is given in appendix (B). The second order system of partial differential equations becomes

$$
\begin{aligned}
\frac{\partial^{2} \Delta \hat{r}_{2}}{\partial T_{0}^{2}}-3 \Delta \hat{r}_{2}-2 \frac{\partial \Delta \hat{\theta}_{2}}{\partial T_{0}}= & \gamma_{r_{2}} \mathrm{e}^{i T_{0}}+\bar{\gamma}_{r_{2}} \mathrm{e}^{-i T_{0}}+\eta_{r_{2}} \mathrm{e}^{2 i T_{0}}+\bar{\eta}_{r_{2}} \mathrm{e}^{-2 i T_{0}}+ \\
& +\chi_{r_{2}} \mathrm{e}^{3 i T_{0}}+\bar{\chi}_{r_{2}} \mathrm{e}^{-3 i T_{0}}+\sigma_{r_{2}}+\lambda_{r_{2}} T_{0} \\
\frac{\partial^{2} \Delta \hat{\theta}_{2}}{\partial T_{0}^{2}}+2 \frac{\partial \Delta \hat{r}_{2}}{\partial T_{0}}= & \gamma_{\theta_{2}} \mathrm{e}^{i T_{0}}+\bar{\gamma}_{\theta_{2}} \mathrm{e}^{-i T_{0}}+\eta_{\theta_{2}} \mathrm{e}^{2 i T_{0}}+\bar{\eta}_{\theta_{2}} \mathrm{e}^{-2 i T_{0}}+ \\
& +\chi_{\theta_{2}} \mathrm{e}^{3 i T_{0}}+\bar{\chi}_{\theta_{2}} \mathrm{e}^{-3 i T_{0}}+\sigma_{\theta_{2}} \\
\frac{\partial^{2} \Delta \hat{\phi}_{2}}{\partial T_{0}^{2}}+\Delta \hat{\phi}_{2}= & \gamma_{\phi_{2}} \mathrm{e}^{i T_{0}}+\bar{\gamma}_{\phi_{2}} \mathrm{e}^{-i T_{0}}+\eta_{\phi_{2}} \mathrm{e}^{2 i T_{0}}+\bar{\eta}_{\phi_{2}} \mathrm{e}^{-2 i T_{0}}+ \\
& +\chi_{\phi_{2}} \mathrm{e}^{3 i T_{0}}+\bar{\chi}_{\phi_{2}} \mathrm{e}^{-3 i T_{0}}+\sigma_{\phi_{2}}
\end{aligned}
$$

where

$$
\begin{aligned}
& \gamma_{r_{2}}=\frac{21}{2} A^{3}+\frac{19}{4} A^{2} B-19 A B^{2}+\frac{7}{2} A D^{2}-\frac{1}{4} B D^{2}-6 A^{2} \bar{A}+\frac{5}{2} A \bar{A} B-5 \bar{A} B^{2}- \\
& -6 \bar{A} D^{2}+\frac{21}{2} A \bar{A}^{2}-\frac{13}{4} \bar{A}^{2} B-8 A D \bar{D}+\frac{7}{2} A \bar{D}^{2}-\frac{13}{4} B \bar{D}^{2}+\frac{7}{2} \Delta \hat{v}_{r_{10}} B+ \\
& +\frac{3}{2} \Delta \hat{\omega}_{\theta_{10}} A+\frac{7}{4} \Delta \hat{\omega}_{\theta_{10}} B+2 i \frac{\partial A}{\partial T_{2}} \\
& \gamma_{\theta_{2}}=\left(4 B^{2} \bar{A}-\frac{15}{2} A^{3}-\frac{47}{4} A^{2} B-\frac{35}{4} A B^{2}-\frac{5}{2} A D^{2}-\frac{1}{4} B D^{2}-6 A^{2} \bar{A}-\frac{19}{2} A \bar{A} B+\right. \\
& +6 \bar{A} D^{2}-\frac{15}{2} A \bar{A}^{2}+\frac{11}{4} \bar{A}^{2} B-\frac{5}{2} A D^{2}+\frac{11}{4} B D^{2}+\frac{3}{2} \Delta \hat{\omega}_{\theta} A-\frac{5}{4} \Delta \hat{\omega}_{\theta_{10}} B- \\
& \left.-\frac{5}{2} i \Delta \hat{v}_{r_{10}} B\right) i+2 \frac{\partial A}{\partial T_{2}} \\
& \gamma_{\phi_{2}}=2 A \bar{A} D-\frac{9}{2} A^{2} D-\frac{33}{2} A B D-\frac{3}{2} B^{2} D-\frac{3}{2} D^{3}-\frac{21}{2} \bar{A} B D-\frac{9}{2} \bar{A}^{2} D+2 A^{2} D- \\
& -3 A B \bar{D}-2 D^{2} \bar{D}-9 \bar{A} B \bar{D}-\frac{3}{2} D \bar{D}^{2}+\frac{3}{2} \Delta \hat{\omega}_{\theta_{10}} D-\frac{3}{2} i \Delta \hat{\omega}_{\phi_{10}}-2 i \frac{\partial D}{\partial T_{2}} \\
& \eta_{r_{2}}=4 A D \bar{D}+4 \bar{A} \bar{D} D-\frac{3}{2} A \bar{D}^{2}-\frac{27}{2} A^{3}-12 A^{2} B-\frac{27}{2} A D^{2}-B D^{2}- \\
& -3 A \bar{A} B+4 \bar{A} D^{2}-\frac{3}{2} A \bar{A}^{2}+\frac{3}{2} \Delta \hat{\omega}_{\theta_{10}} A+2 i \Delta \hat{\omega}_{\phi_{10}} D+3 i \Delta \hat{v}_{r_{10}} A \\
& \eta_{\theta_{2}}=\left(27 A^{3}+A^{2} B+15 A D^{2}+3 B D^{2}+6 A \bar{A} B-4 \bar{A} D^{2}+3 A \bar{A}^{2}-4 A D \bar{D}-\right. \\
& \left.-4 \bar{A} \bar{D} D+3 A \bar{D}^{2}-3 \Delta \hat{\omega}_{\theta_{10}} A\right) i-2 \Delta \hat{v}_{r_{10}} A+2 \Delta \hat{\omega}_{\phi_{10}} D
\end{aligned}
$$




$$
\begin{aligned}
\eta_{\phi_{2}}= & \frac{63}{2} A^{2} D+5 A B D+\frac{3}{2} D^{3}-6 A \bar{A} D+3 \bar{A} B D+\frac{3}{2} \bar{A}^{2} D-6 A^{2} \bar{D}- \\
& -6 A \bar{A} \bar{D}+\frac{3}{2} D \bar{D}^{2}-\frac{3}{2} \Delta \hat{\omega}_{\theta_{10}} D-3 i \Delta \hat{\omega}_{\phi_{10}} A-3 i \Delta \hat{v}_{r_{10}} D \\
\chi_{r_{2}}= & 10 A D^{2} \\
\chi_{\theta_{2}}= & -\left(24 A^{3}+14 A D^{2}\right) i \\
\chi_{\phi_{2}}= & -30 A^{2} D-2 D^{3} \\
\sigma_{r_{2}}= & 2 \frac{\partial C}{\partial T_{2}}+\frac{9}{4} A^{2} B-\frac{3}{2} A^{3}+\frac{45}{4} A B^{2}-\frac{15}{8} B^{3}-\frac{3}{2} A D^{2}+\frac{27}{4} B D^{2}-\frac{27}{2} A^{2} \bar{A}- \\
& -32 A \bar{A} B+\frac{45}{4} \bar{A} B^{2}-\frac{3}{2} \bar{A} D^{2}-\frac{27}{2} A \bar{A}^{2}-\frac{3}{4} \bar{A}^{2} B-\frac{3}{2} \bar{A}^{3}-6 B D \bar{D}-\frac{3}{2} A \bar{D}^{2}+ \\
& +\frac{27}{4} B \bar{D}^{2}-\frac{3}{2} \bar{A} \bar{D}^{2}-3 i A \Delta \hat{v}_{r_{10}}+3 i \Delta \hat{v}_{r_{10}} \bar{A}+\frac{3}{2} \Delta \hat{\omega}_{\theta_{10}} A+\frac{3}{2} \Delta \hat{\omega}_{\theta_{10}} \bar{A}+ \\
& +\frac{3}{4} \Delta \hat{\omega}_{\theta_{10}} B \\
& +4 A \bar{A} \bar{D}+\bar{A} B \bar{D}-\frac{3}{2} \bar{A}^{2} \bar{D}+\frac{1}{2} D \bar{D}^{2}+\frac{1}{2} \bar{D}^{3}-\frac{1}{2} \Delta \hat{\omega}_{\theta_{10}}-\frac{1}{2} \Delta \hat{\omega}_{\theta_{10}} \bar{D}+ \\
& i \Delta \hat{v}_{r_{10}} D-i \Delta \hat{v}_{r_{10}} \bar{D}+i \Delta \hat{\omega}_{\phi_{10}} A-i \Delta \hat{\omega}_{\phi_{10}} \bar{A} \\
\lambda_{r_{2}}= & -3 \frac{\partial B}{\partial T_{2}} \\
\sigma_{\phi_{2}}= & \left.4 \frac{3}{4} A^{2} B+\frac{33}{4} A B^{2}-\frac{3}{4} B D^{2}-3 A \bar{A} B-\frac{33}{4} \bar{A} B^{2}+\frac{9}{4} \bar{A}^{2} B+\frac{3}{4} B \bar{D}^{2}\right) i+\frac{\partial B}{\partial T_{2}} \\
& \\
&
\end{aligned}
$$

In a similar manner as the first order solution, the Laplace transforms of $\Delta \hat{r}_{2}$ and $\Delta \hat{\theta}_{2}$ are

$$
\begin{aligned}
\Delta \hat{R}_{2}= & \frac{\gamma_{r_{2}}}{\left(s^{2}+1\right)(s-i)}+\frac{\bar{\gamma}_{r_{2}}}{\left(s^{2}+1\right)(s+i)}+\frac{\eta_{r_{2}}}{\left(s^{2}+1\right)(s-2 i)}+\frac{\bar{\eta}_{r_{2}}}{\left(s^{2}+1\right)(s+2 i)}+ \\
& +\frac{\chi_{r_{2}}}{\left(s^{2}+1\right)(s-3 i)}+\frac{\bar{\chi}_{r_{2}}}{\left(s^{2}+1\right)(s+3 i)}+\frac{\lambda_{r_{2}}+2 \sigma_{\theta_{2}}}{s^{2}\left(s^{2}+1\right)}+\frac{\sigma_{r_{2}}}{s\left(s^{2}+1\right)}+ \\
& +\frac{2 \gamma_{\theta_{2}}}{s\left(s^{2}+1\right)(s-i)}+\frac{2 \bar{\gamma}_{\theta_{2}}}{s\left(s^{2}+1\right)(s+i)}+\frac{2 \eta_{\theta_{2}}}{s\left(s^{2}+1\right)(s-2 i)}+ \\
& +\frac{2 \bar{\eta}_{\theta_{2}}}{s\left(s^{2}+1\right)(s+2 i)}+\frac{2 \chi_{\theta_{2}}}{s\left(s^{2}+1\right)(s-3 i)}+\frac{2 \bar{\chi}_{\theta_{2}}}{s\left(s^{2}+1\right)(s+3 i)}+
\end{aligned}
$$




$$
\begin{aligned}
& +\frac{\Delta \hat{v}_{r_{20}}}{s^{2}+1}+\frac{2 \Delta \hat{\omega}_{\theta_{20}}}{s\left(s^{2}+1\right)} \\
\Delta \hat{\Theta}_{2}= & -\frac{2 \gamma_{r_{2}}}{s\left(s^{2}+1\right)(s-i)}-\frac{2 \bar{\gamma}_{r_{2}}}{s\left(s^{2}+1\right)(s+i)}-\frac{2 \eta_{r_{2}}}{s\left(s^{2}+1\right)(s-2 i)}-\frac{2 \bar{\eta}_{r_{2}}}{s\left(s^{2}+1\right)(s+2 i)}- \\
& -\frac{2 \chi_{r_{2}}}{s\left(s^{2}+1\right)(s-3 i)}-\frac{2 \bar{\chi}_{r_{2}}}{s\left(s^{2}+1\right)(s+3 i)}-\frac{2 \lambda_{r_{2}}}{s^{3}\left(s^{2}+1\right)}-\frac{2 \sigma_{r_{2}}}{s^{2}\left(s^{2}+1\right)}+ \\
& +\frac{\gamma_{\theta_{2}}\left(s^{2}-3\right)}{s^{2}\left(s^{2}+1\right)(s-i)}+\frac{\bar{\gamma}_{\theta_{2}}\left(s^{2}-3\right)}{s^{2}\left(s^{2}+1\right)(s+i)}+\frac{\eta_{\theta_{2}}\left(s^{2}-3\right)}{s^{2}\left(s^{2}+1\right)(s-2 i)}+ \\
& +\frac{\bar{\eta}_{\theta_{2}}\left(s^{2}-3\right)}{s^{2}\left(s^{2}+1\right)(s+2 i)}+\frac{\chi_{\theta_{2}}\left(s^{2}-3\right)}{s^{2}\left(s^{2}+1\right)(s-3 i)}+\frac{\bar{\chi}_{\theta_{2}}\left(s^{2}-3\right)}{s^{2}\left(s^{2}+1\right)(s+3 i)}+ \\
& +\frac{\sigma_{\theta_{2}}\left(s^{2}-3\right)}{s^{3}\left(s^{2}+1\right)}+\frac{\left(s^{2}-3\right) \Delta \hat{\omega}_{\theta_{20}}}{s^{2}\left(s^{2}+1\right)}-\frac{2 \Delta \hat{v}_{r_{20}}}{s\left(s^{2}+1\right)}
\end{aligned}
$$

The new inverse Laplace transforms are

$$
\begin{aligned}
\mathcal{L}^{-1}\left[\frac{1}{\left(s^{2}+1\right)(s \pm 3 i)}\right] & =\frac{1}{4} \mathrm{e}^{\mp i T_{0}}-\frac{1}{8} \mathrm{e}^{ \pm i T_{0}}-\frac{1}{8} \mathrm{e}^{\mp 3 i T_{0}} \\
\mathcal{L}^{-1}\left[\frac{1}{s\left(s^{2}+1\right)(s \pm 3 i)}\right] & = \pm i\left(\frac{1}{4} \mathrm{e}^{\mp i T_{0}}+\frac{1}{8} \mathrm{e}^{ \pm i T_{0}}-\frac{1}{24} \mathrm{e}^{\mp 3 i T_{0}}-\frac{1}{3}\right) \\
\mathcal{L}^{-1}\left[\frac{1}{s^{2}\left(s^{2}+1\right)(s \pm 3 i)}\right] & =\frac{1}{9}-\frac{1}{4} \mathrm{e}^{\mp i T_{0}}+\frac{1}{8} \mathrm{e}^{ \pm i T_{0}}+\frac{1}{72} \mathrm{e}^{\mp 3 i T_{0}} \mp \frac{1}{3} i T_{0}
\end{aligned}
$$

which leads to

$$
\begin{aligned}
\Delta \hat{r}_{2}= & \left(\frac{\gamma_{r_{2}}}{4}+\frac{\eta_{r_{2}}}{2}-\frac{\bar{\gamma}_{r_{2}}}{4}+\frac{i \bar{\gamma}_{\theta_{2}}}{2}-\frac{\bar{\eta}_{r_{2}}}{6}+\frac{i \bar{\eta}_{\theta_{2}}}{3}-\frac{3 i \gamma_{\theta_{2}}}{2}-i \eta_{\theta_{2}}+\frac{i \lambda_{r_{2}}}{2}-\frac{\sigma_{r_{2}}}{2}+i \sigma_{\theta_{2}}-\right. \\
& \left.-\frac{\bar{\chi}_{r_{2}}}{8}+\frac{\chi_{r_{2}}}{4}-\frac{i \chi_{\theta_{2}}}{2}+\frac{i \bar{\chi}_{\theta_{2}}}{4}-\frac{i \Delta \hat{v}_{r_{20}}}{2}-\Delta \hat{\omega}_{\theta_{20}}-\left[\gamma_{\theta_{2}}+\frac{i \gamma_{r_{2}}}{2}\right] T_{0}\right) \mathrm{e}^{i T_{0}}+ \\
& +\left(\frac{\bar{\gamma}_{r_{2}}}{4}+\frac{\bar{\eta}_{r_{2}}}{2}-\frac{\gamma_{r_{2}}}{4}-\frac{i \gamma_{\theta_{2}}}{2}-\frac{\eta_{r_{2}}}{6}-\frac{i \eta_{\theta_{2}}}{3}+\frac{3 i \bar{\gamma}_{\theta_{2}}}{2}+i \bar{\eta}_{\theta_{2}}-\frac{i \lambda_{r_{2}}}{2}-\frac{\sigma_{r_{2}}}{2}-i \sigma_{\theta_{2}}-\right. \\
& \left.-\frac{\chi_{r_{2}}}{8}+\frac{\bar{\chi}_{r_{2}}}{4}+\frac{i \bar{\chi}_{\theta_{2}}}{2}-\frac{i \chi_{\theta_{2}}}{4}+\frac{i \Delta \hat{v}_{r_{20}}}{2}-\Delta \hat{\omega}_{\theta_{20}}-\left[\bar{\gamma}_{\theta_{2}}-\frac{i \bar{\gamma}_{r_{2}}}{2}\right] T_{0}\right) \mathrm{e}^{-i T_{0}}+ \\
& +\left(\frac{i \eta_{\theta_{2}}}{3}-\frac{\eta_{r_{2}}}{3}\right) \mathrm{e}^{2 i T_{0}}+\left(-\frac{i \bar{\eta}_{\theta_{2}}}{3}-\frac{\bar{\eta}_{r_{2}}}{3}\right) \mathrm{e}^{-2 i T_{0}}+ \\
& +\left(\frac{i \chi_{\theta_{2}}}{12}-\frac{\chi_{r_{2}}}{8}\right) \mathrm{e}^{3 i T_{0}}+\left(-\frac{i \bar{\chi}_{\theta_{2}}}{12}-\frac{\bar{\chi}_{r_{2}}}{8}\right) \mathrm{e}^{-3 i T_{0}}+\left(\lambda_{r_{2}}+2 \sigma_{\theta_{2}}\right) T_{0}+2 i \gamma_{\theta_{2}}- \\
& -2 i \bar{\gamma}_{\theta_{2}}+i \eta_{\theta_{2}}-i \bar{\eta}_{\theta_{2}}+\sigma_{r_{2}}+\frac{2 i \chi_{\theta_{2}}}{3}-\frac{2 i \bar{\chi}_{\theta_{2}}}{3}+2 \Delta \hat{\omega}_{\theta_{20}} \\
\Delta \hat{\theta}_{2}= & \left(4 \gamma_{\theta_{2}}+i \eta_{r_{2}}+2 \eta_{\theta_{2}}-\lambda_{r_{2}}-i \sigma_{r_{2}}-2 \sigma_{\theta_{2}}-\frac{i \bar{\gamma}_{r_{2}}}{2}-\bar{\gamma}_{\theta_{2}}-\frac{i \bar{\eta}_{r_{2}}}{3}-\frac{2 \bar{\eta}_{\theta_{2}}}{3}+\frac{3 i \gamma_{r_{2}}}{2}+\right. \\
& \left.+\chi_{\theta_{2}}-\frac{\bar{\chi}_{\theta_{2}}}{2}+\frac{i \chi_{r_{2}}}{2}-\frac{i \bar{\chi}_{r_{2}}}{4}+\Delta \hat{v}_{r_{20}}-2 i \Delta \hat{\omega}_{\theta_{20}}+\left[\gamma_{r_{2}}-2 i \gamma_{\theta_{2}}\right] T_{0}\right) \mathrm{e}^{i T_{0}}+
\end{aligned}
$$




$$
\begin{aligned}
& +\left(4 \bar{\gamma}_{\theta_{2}}-i \bar{\eta}_{r_{2}}+2 \bar{\eta}_{\theta_{2}}-\lambda_{r_{2}}+i \sigma_{r_{2}}-2 \sigma_{\theta_{2}}+\frac{i \gamma_{r_{2}}}{2}-\gamma_{\theta_{2}}+\frac{i \eta_{r_{2}}}{3}-\frac{2 \eta_{\theta_{2}}}{3}-\frac{3 i \bar{\gamma}_{r_{2}}}{2}+\right. \\
& \left.+\bar{\chi}_{\theta_{2}}-\frac{\chi_{\theta_{2}}}{2}-\frac{i \bar{\chi}_{r_{2}}}{2}+\frac{i \chi_{r_{2}}}{4}+\Delta \hat{v}_{r_{20}}+2 i \Delta \hat{\omega}_{\theta_{20}}+\left[\bar{\gamma}_{r_{2}}+2 i \bar{\gamma}_{\theta_{2}}\right] T_{0}\right) \mathrm{e}^{-i T_{0}}+ \\
& +\left(-\frac{i \eta_{r_{2}}}{3}-\frac{7 \eta_{\theta_{2}}}{12}\right) \mathrm{e}^{2 i T_{0}}+\left(\frac{i \bar{\eta}_{r_{2}}}{3}-\frac{7 \bar{\eta}_{\theta_{2}}}{12}\right) \mathrm{e}^{-2 i T_{0}}+ \\
& +\left(-\frac{i \chi_{r_{2}}}{12}-\frac{\chi_{\theta_{2}}}{6}\right) \mathrm{e}^{3 i T_{0}}+\left(\frac{i \bar{\chi}_{r_{2}}}{12}-\frac{\bar{\chi}_{\theta_{2}}}{6}\right) \mathrm{e}^{-3 i T_{0}}+ \\
& +\left(3 i \bar{\gamma}_{\theta_{2}}-3 i \gamma_{\theta_{2}}+\frac{3 i \bar{\eta}_{\theta_{2}}}{2}-\frac{3 i \eta_{\theta_{2}}}{2}-2 \sigma_{r_{2}}-i \chi_{\theta_{2}}+i \bar{\chi}_{\theta_{2}}-3 \Delta \hat{\omega}_{\theta_{20}}\right) T_{0}- \\
& -\left(\lambda_{r_{2}}+\frac{3 \sigma_{\theta_{2}}}{2}\right) T_{0}^{2}+2 i \bar{\gamma}_{r_{2}}-2 i \gamma_{r_{2}}-3 \bar{\gamma}_{\theta_{2}}-3 \gamma_{\theta_{2}}+i \bar{\eta}_{r_{2}}-i \eta_{r_{2}}- \\
& -\frac{3 \bar{\eta}_{\theta_{2}}}{4}-\frac{3 \eta_{\theta_{2}}}{4}+2 \lambda_{r_{2}}+4 \sigma_{\theta_{2}}+\frac{2 i \bar{\chi}_{r_{2}}}{3}-\frac{2 i \bar{\chi}_{r_{2}}}{3}-\frac{\chi_{\theta_{2}}}{3}-\frac{\bar{\chi}_{\theta_{2}}}{3}-2 \Delta \hat{v}_{r_{20}}
\end{aligned}
$$

Eliminating secular terms from the solution requires that

$$
\begin{gathered}
\gamma_{r_{2}}-2 i \gamma_{\theta_{2}}=0 \\
\lambda_{r_{2}}+2 \sigma_{\theta_{2}}=0 \\
\lambda_{r_{2}}+\frac{3 \sigma_{\theta_{2}}}{2}=0
\end{gathered}
$$

and in order to satisfy the initial conditions it must be true that

$$
3 i \bar{\gamma}_{\theta_{2}}-3 i \gamma_{\theta_{2}}+\frac{3 i \bar{\eta}_{\theta_{2}}}{2}-\frac{3 i \eta_{\theta_{2}}}{2}-2 \sigma_{r_{2}}-i \chi_{\theta_{2}}+i \bar{\chi}_{\theta_{2}}-3 \Delta \hat{\omega}_{\theta_{20}}=0
$$

Expanding equation (4.125) results in

$$
\begin{aligned}
\frac{\partial A}{\partial T_{2}}= & i\left(\frac{9}{4} A^{3}+\frac{93}{8} A^{2} B+\frac{73}{4} A B^{2}+\frac{3}{4} A D^{2}+\frac{3}{8} B D^{2}+9 A^{2} \bar{A}+\frac{33}{4} A \bar{A} B-\right. \\
& -\frac{3}{2} B^{2} \bar{A}-3 \bar{A} D^{2}+\frac{9}{4} A \bar{A}^{2}-\frac{9}{8} \bar{A}^{2} B+4 A D \bar{D}+\frac{3}{4} A \bar{D}^{2}-\frac{9}{8} B \bar{D}^{2}- \\
& \left.-\frac{9}{4} A \Delta \hat{\omega}_{\theta_{10}}+\frac{3}{8} B \Delta \hat{\omega}_{\theta_{10}}\right)-\frac{3}{4} B \Delta \hat{v}_{r_{10}}
\end{aligned}
$$

Equation (4.128) leads to

$$
\begin{aligned}
\frac{\partial C}{\partial T_{2}}= & \frac{3}{4} A^{2}+\frac{3}{4} A^{3}+\frac{9}{2} A B-\frac{51}{8} A^{2} B-\frac{15}{8} A B^{2}+\frac{15}{16} B^{3}+\frac{3}{4} D^{2}+\frac{3}{4} A D^{2}- \\
& -\frac{27}{8} B D^{2}+\frac{27}{4} A^{2} \bar{A}+\frac{9}{2} \bar{A} B+13 A \bar{A} B-\frac{15}{8} \bar{A} B^{2}+\frac{3}{4} \bar{A} D^{2}+\frac{3}{4} \bar{A}^{2}+ \\
& +\frac{27}{4} A \bar{A}^{2}-\frac{51}{8} \bar{A}^{2} B+\frac{3}{4} \bar{A}^{3}+\frac{3}{4} \bar{D}^{2}+3 B D \bar{D}+\frac{3}{4} A \bar{D}^{2}-\frac{27}{2} B \bar{D}^{2}+ \\
& +\frac{3}{4} \bar{A} \bar{D}^{2}-\frac{27}{4} A \Delta \hat{\omega}_{\theta_{10}}-\frac{27}{4} \bar{A} \Delta \hat{\omega}_{\theta_{10}}+\frac{9}{8} B \Delta \hat{\omega}_{\theta_{10}}-\frac{3}{4} \Delta \hat{\omega}_{\theta_{20}}+ \\
& +\frac{9}{8} i B \Delta \hat{v}_{r_{10}}-\frac{9}{8} i B \Delta \hat{v}_{r_{10}}
\end{aligned}
$$


Equations (4.126) and (4.127) again imply that $\lambda_{r_{2}}$ and $\sigma_{\theta_{2}}$ must both be equal to 0 . However, expanding each of these variables results in

$$
\frac{\partial B}{\partial T_{2}}=0
$$

and

$$
\frac{\partial B}{\partial T_{2}}=i\left(\frac{3}{4} B D^{2}+3 A \bar{A} B+\frac{33}{4} \bar{A} B^{2}-\frac{3}{4} A^{2} B-\frac{33}{4} A B^{2}-\frac{9}{4} \bar{A}^{2} B-\frac{3}{4} B \bar{D}^{2}\right)
$$

Equations (4.131) and (4.132) contradict each other, and therefore the method of multiple scales cannot remove secular terms from this expansion at order $\epsilon^{2}$. Continuing on to the out-of-plane equation for completeness gives

$$
\begin{aligned}
\Delta \hat{\Phi}_{2}= & \frac{\gamma_{\phi_{2}}}{\left(s^{2}+1\right)(s-i)}+\frac{\bar{\gamma}_{\phi_{2}}}{\left(s^{2}+1\right)(s+i)}+\frac{\eta_{\phi_{2}}}{\left(s^{2}+1\right)(s-2 i)}+\frac{\bar{\eta}_{\phi_{2}}}{\left(s^{2}+1\right)(s+2 i)}+ \\
& +\frac{\sigma_{\phi_{2}}}{s\left(s^{2}+1\right)}+\frac{\chi_{\phi_{2}}}{\left(s^{2}+1\right)(s-3 i)}+\frac{\bar{\chi}_{\phi_{2}}}{\left(s^{2}+1\right)(s+3 i)}+\frac{\Delta \hat{\omega}_{\phi_{20}}}{s^{2}+1}
\end{aligned}
$$

The inverse transformation is

$$
\begin{aligned}
\Delta \hat{\phi}_{2}= & \left(\frac{\gamma_{\phi_{2}}}{4}+\frac{\eta_{\phi_{2}}}{2}-\frac{\bar{\gamma}_{\phi_{2}}}{4}-\frac{\bar{\eta}_{\phi_{2}}}{6}-\frac{\sigma_{\phi_{2}}}{2}+\frac{\chi_{\phi_{2}}}{4}-\frac{\bar{\chi}_{\phi_{2}}}{8}-\frac{i \Delta \hat{\omega}_{\phi_{20}}}{2}-\frac{i \gamma_{\phi_{2}}}{2} T_{0}\right) \mathrm{e}^{i T_{0}}+ \\
& +\left(\frac{\bar{\gamma}_{\phi_{1}}}{4}+\frac{\bar{\eta}_{\phi_{1}}}{2}-\frac{\gamma_{\phi_{1}}}{4}-\frac{\eta_{\phi_{1}}}{6}-\frac{\sigma_{\phi_{1}}}{2}+\frac{\bar{\chi}_{\phi_{2}}}{4}-\frac{\chi_{\phi_{2}}}{8}+\frac{i \Delta \hat{\omega}_{\phi_{20}}}{2}+\frac{i \bar{\gamma}_{\phi_{1}}}{2} T_{0}\right) \mathrm{e}^{-i T_{0}}- \\
& -\frac{\eta_{\phi_{1}}}{3} \mathrm{e}^{2 i T_{0}}-\frac{\bar{\eta}_{\phi_{1}}}{3} \mathrm{e}^{-2 i T_{0}}-\frac{\chi_{\phi_{2}}}{8} \mathrm{e}^{3 i T_{0}}-\frac{\bar{\chi}_{\phi_{2}}}{8} \mathrm{e}^{-3 i T_{0}}+\sigma_{\phi_{1}}
\end{aligned}
$$

In order to eliminate secular terms, it must be true that

$$
\gamma_{\phi_{2}}=0
$$

or, that

$$
\begin{aligned}
\frac{\partial D}{\partial T_{2}}= & i\left(\frac{3}{4} D \bar{D}^{2}+\frac{9}{4} \bar{A} B \bar{D}+D^{2} \bar{D}+\frac{3}{4} A B \bar{D}-A^{2} D+\frac{9}{4} \bar{A}^{2} D+\frac{21}{4} \bar{A} B D+\right. \\
& \left.+\frac{3}{4} D^{3}+\frac{3}{4} B^{2} D+\frac{33}{4} A B D+\frac{9}{4} A^{2} D-A \bar{A} D-\frac{3}{2} \Delta \hat{\omega}_{\theta_{10}}\right)-\frac{3}{2} \Delta \hat{\omega}_{\phi_{10}}
\end{aligned}
$$

This section has attempted to find uniform solutions for $\Delta \hat{r}_{2}, \Delta \hat{\theta}_{2}$ and $\Delta \hat{\phi}_{2}$ which would normally form the second term in the approximation of (4.10), (4.11) and (4.12). Unfortunately, the conditions placed on the variable $B$ were contradictory, and as a result uniform solutions can not be found, meaning that the approximate solution of (4.10), (4.11) and (4.12) is limited to corrections of order $\epsilon$ only. 


\subsubsection{Solvability Conditions}

This section will solve the conditions placed upon variables $A, B, C$ and $D$ that were determined in the previous sections such that the correction of order $\epsilon$ was free of secular terms. Restating those conditions here to summarize, it was found that

$$
\begin{aligned}
& \frac{\partial A}{\partial T_{1}}=-\frac{3}{2} i A B \\
& \frac{\partial B}{\partial T_{1}}=0 \\
& \frac{\partial C}{\partial T_{1}}=\frac{15}{8} B^{2}+3 A \bar{A}+\frac{3}{4}\left(D^{2}+\bar{D}^{2}\right)+\frac{9}{4}\left(A^{2}+\bar{A}^{2}\right)+\frac{3}{2}(A B+\bar{A} B)-\frac{3}{2} \Delta \hat{\omega}_{\theta_{10}} \\
& \frac{\partial D}{\partial T_{1}}=-\frac{3}{2} i D B
\end{aligned}
$$

First, equation (4.138) states that $B$ is a constant, or

$$
B=b_{0}
$$

Next, using the polar transformation

$$
A=\frac{1}{2} a \mathrm{e}^{i \alpha}
$$

and the result from (4.141) applied to equation (4.137) leads to

$$
\frac{1}{2} \frac{\partial a}{\partial T_{1}} \mathrm{e}^{i \alpha}+\frac{1}{2} i a \frac{\partial \alpha}{\partial T_{1}} \mathrm{e}^{i \alpha}=-\frac{3}{4} i a b_{0} \mathrm{e}^{i \alpha}
$$

Multiplying through by $2 \mathrm{e}^{-i \alpha}$ leads to

$$
\frac{\partial a}{\partial T_{1}}+i a \frac{\partial \alpha}{\partial T_{1}}=-\frac{3}{2} i a b_{0}
$$

Equating real and imaginary parts,

$$
\begin{aligned}
& \frac{\partial a}{\partial T_{1}}=0 \\
& \frac{\partial \alpha}{\partial T_{1}}=-\frac{3}{2} b_{0}
\end{aligned}
$$

or, that

$$
\begin{aligned}
a & =a_{0} \\
\alpha & =-\frac{3}{2} b_{0} T_{1}+\alpha_{0}
\end{aligned}
$$

Using a similar transformation on variable $D$,

$$
D=\frac{1}{2} d \mathrm{e}^{i \delta}
$$


on equation (4.140) leads to

$$
\frac{1}{2} \frac{\partial d}{\partial T_{1}} \mathrm{e}^{i \delta}+\frac{1}{2} i d \frac{\partial \delta}{\partial T_{1}} \mathrm{e}^{i \delta}=-\frac{3}{4} i d b_{0} \mathrm{e}^{i \delta}
$$

Multiplying through by $2 \mathrm{e}^{-i \delta}$ and equating real and imaginary parts gives

$$
\begin{aligned}
& \frac{\partial d}{\partial T_{1}}=0 \\
& \frac{\partial \delta}{\partial T_{1}}=-\frac{3}{2} b_{0}
\end{aligned}
$$

which leads to

$$
\begin{aligned}
d & =d_{0} \\
\delta & =-\frac{3}{2} b_{0} T_{1}+\delta_{0}
\end{aligned}
$$

Substituting these results into equation (4.139) gives

$$
\begin{aligned}
\frac{\partial C}{\partial T_{1}}= & \frac{15}{8} b_{0}^{2}+\frac{3}{4} a_{0}^{2}+\frac{3}{16} d_{0}^{2}\left(\mathrm{e}^{2 i \delta}+\mathrm{e}^{-2 i \delta}\right)+\frac{9}{16} a_{0}^{2}\left(\mathrm{e}^{2 i \alpha}+\mathrm{e}^{-2 i \alpha}\right)+ \\
& +\frac{3}{4} a_{0} b_{0}\left(\mathrm{e}^{i \alpha}+\mathrm{e}^{-i \alpha}\right)-\frac{3}{4} \Delta \hat{\omega}_{\theta_{10}} \\
= & \frac{15}{8} b_{0}^{2}+\frac{3}{4} a_{0}^{2}+\frac{3}{8} d_{0}^{2} \cos 2 \delta+\frac{9}{8} a_{0}^{2} \cos 2 \alpha+\frac{3}{2} a_{0} b_{0} \cos \alpha-\frac{3}{4} \Delta \hat{\omega}_{\theta_{10}} \\
= & \frac{15}{8} b_{0}^{2}+\frac{3}{4} a_{0}^{2}+\frac{3}{8} d_{0}^{2} \cos \left(2 \delta_{0}-3 b_{0} T_{1}\right)+\frac{9}{8} a_{0}^{2} \cos \left(2 \alpha_{0}-3 b_{0} T_{1}\right)+ \\
& +\frac{3}{2} a_{0} b_{0} \cos \left(\alpha_{0}-\frac{3}{2} b_{0} T_{1}\right)-\frac{3}{4} \Delta \hat{\omega}_{\theta_{10}}
\end{aligned}
$$

If $b_{0} \neq 0$, then

$$
\begin{aligned}
C= & \left(\frac{15}{8} b_{0}^{2}+\frac{3}{4} a_{0}^{2}-\frac{3}{4} \Delta \hat{\omega}_{\theta_{10}}\right) T_{1}-\frac{3}{8} \frac{a_{0}^{2}}{b_{0}} \sin \left(2 \alpha_{0}-3 b_{0} T_{1}\right)-a_{0} \sin \left(\alpha 0-\frac{3}{2} b_{0} T_{1}\right)- \\
& -\frac{1}{8} \frac{d_{0}^{2}}{b_{0}} \sin \left(2 \delta_{0}-3 b_{0} T_{1}\right)+Q
\end{aligned}
$$

where $Q$ is a constant of integration that can be found by

$$
\begin{aligned}
C(0) & =Q-\frac{3}{8} \frac{a_{0}^{2}}{b_{0}} \sin 2 \alpha_{0}-a_{0} \sin \alpha_{0}-\frac{1}{8} \frac{d_{0}^{2}}{b_{0}} \sin 2 \delta_{0} \\
& =c_{0}
\end{aligned}
$$

which leads to

$$
Q=c_{0}+\frac{1}{8} \frac{d_{0}^{2}}{b_{0}} \sin 2 \delta_{0}+\frac{3}{8} \frac{a_{0}^{2}}{b_{0}} \sin 2 \alpha_{0}+a_{0} \sin \alpha_{0}
$$


So,

$$
\begin{aligned}
C= & \left(\frac{15}{8} b_{0}^{2}+\frac{3}{4} a_{0}^{2}-\frac{3}{4} \Delta \hat{\omega}_{\theta_{10}}\right) T_{1}+\frac{1}{8} \frac{d_{0}^{2}}{b_{0}}\left[\sin \left(3 b_{0} T_{1}-2 \delta_{0}\right)+\sin 2 \delta_{0}\right]+ \\
& +\frac{3}{8} \frac{a_{0}^{2}}{b_{0}}\left[\sin \left(3 b_{0} T_{1}-2 \alpha_{0}\right)+\sin 2 \alpha_{0}\right]+a_{0}\left[\sin \left(\frac{3}{2} b_{0} T_{1}-\alpha_{0}\right)+\sin \alpha_{0}\right]+c_{0}
\end{aligned}
$$

If $b_{0}=0$, then

$$
\frac{\partial C}{\partial T_{1}}=\frac{3}{4} a_{0}^{2}+\frac{3}{8} d_{0}^{2} \cos 2 \delta_{0}+\frac{9}{8} a_{0}^{2} \cos 2 \alpha_{0}-\frac{3}{4} \Delta \hat{\omega}_{\theta_{10}}
$$

which leads to

$$
C=\left(\frac{3}{4} a_{0}^{2}+\frac{3}{8} d_{0}^{2} \cos 2 \delta_{0}+\frac{9}{8} a_{0}^{2} \cos 2 \alpha_{0}-\frac{3}{4} \Delta \hat{\omega}_{\theta_{10}}\right) T_{1}+c_{0}
$$

At this point, the initial conditions $\Delta \hat{v}_{r_{10}}, \Delta \hat{\omega}_{\phi_{10}}$ and $\Delta \hat{\omega}_{\theta_{10}}$ can be found from equations (4.40), (4.42) and (4.44):

$$
\begin{aligned}
\Delta \hat{v}_{r_{10}}= & -\frac{\partial \Delta r_{0}}{\partial T_{1}}(0) \\
= & -\left[\frac{\partial A}{\partial T_{1}} \mathrm{e}^{i T_{0}}+\frac{\partial \bar{A}}{\partial T_{1}} \mathrm{e}^{-i T_{0}}+\frac{\partial B}{\partial T_{1}}\right](0) \\
= & \frac{3}{4} i a_{0} b_{0}\left(\mathrm{e}^{i \alpha_{0}}-\mathrm{e}^{-i \alpha_{0}}\right) \\
= & -\frac{3}{2} a_{0} b_{0} \sin \alpha_{0} \\
= & -\left[\frac{\partial D}{\partial T_{1}} \mathrm{e}^{i T_{0}}+\frac{\partial \bar{D}}{\partial T_{1}} \mathrm{e}^{-i T_{0}}\right](0) \\
\left.\Delta \hat{\omega}_{\phi_{10}}\right] & \frac{3}{4} i d_{0} b_{0}\left(\mathrm{e}^{i \delta_{0}}-\mathrm{e}^{-i \delta_{0}}\right) \\
= & -\frac{3}{2} d_{0} b_{0} \sin \delta_{0} \\
\Delta \hat{\omega}_{\theta_{10}}= & -\frac{\partial \Delta \hat{\theta}_{0}}{\partial T_{1}}(0) \\
= & -\left[2 i \frac{\partial A}{\partial T_{1}} \mathrm{e}^{i T_{0}}-2 i \frac{\partial \bar{A}}{\partial T_{1}} \mathrm{e}^{-i T_{0}}+\frac{\partial C}{\partial T_{1}}\right](0) \\
= & -\frac{3}{2} a_{0} b_{0}\left(\mathrm{e}^{i \alpha_{0}}+\mathrm{e}^{-i \alpha_{0}}\right)-\frac{15}{8} b_{0}^{2}-\frac{3}{4} a_{0}^{2}-\frac{9}{16} a_{0}^{2}\left(\mathrm{e}^{2 i \alpha_{0}}+\mathrm{e}^{-2 i \alpha_{0}}\right)- \\
& -\frac{3}{4} a_{0} b_{0}\left(\mathrm{e}^{i \alpha_{0}}+\mathrm{e}^{-i \alpha_{0}}\right)-\frac{3}{16} d_{0}^{2}\left(\mathrm{e}^{2 i \delta_{0}}+\mathrm{e}^{-2 i \delta_{0}}\right)+\frac{3}{4} \Delta \hat{\omega}_{\theta_{10}}
\end{aligned}
$$

so,

$$
\Delta \hat{\omega}_{\theta_{10}}=-\frac{15}{2} b_{0}^{2}-3 a_{0}^{2}-9 a_{0} b_{0}\left(\mathrm{e}^{i \alpha_{0}}+\mathrm{e}^{-i \alpha_{0}}\right)-\frac{9}{4} a_{0}^{2}\left(\mathrm{e}^{2 i \alpha_{0}}-\mathrm{e}^{-2 i \alpha_{0}}\right)-
$$




$$
\begin{aligned}
& -\frac{3}{4} d_{0}^{2}\left(\mathrm{e}^{2 i \delta_{0}}+\mathrm{e}^{-2 i \delta_{0}}\right) \\
= & -\frac{15}{2} b_{0}^{2}-3 a_{0}^{2}-18 a_{0} b_{0} \cos \alpha_{0}-\frac{9}{2} a_{0}^{2} \cos 2 \alpha_{0}-\frac{3}{2} d_{0}^{2} \cos 2 \delta_{0}
\end{aligned}
$$

Substituting this result into the equation for $C$ gives, for $b_{0} \neq 0$ that

$$
\begin{aligned}
C= & \left(\frac{15}{2} b_{0}^{2}+3 a_{0}^{2}+\frac{27}{2} a_{0} b_{0} \cos \alpha_{0}+\frac{27}{8} \cos 2 \alpha_{0}+\frac{9}{8} \cos 2 \delta_{0}\right) T_{1}+ \\
& +\frac{1}{8} \frac{d_{0}^{2}}{b_{0}}\left[\sin \left(3 b_{0} T_{1}-2 \delta_{0}\right)+\sin 2 \delta_{0}\right]+\frac{3}{8} \frac{a_{0}^{2}}{b_{0}}\left[\sin \left(3 b_{0} T_{1}-2 \alpha_{0}\right)+\sin 2 \alpha_{0}\right]+ \\
& +a_{0}\left[\sin \left(\frac{3}{2} b_{0} T_{1}-\alpha_{0}\right)+\sin \alpha_{0}\right]+c_{0}
\end{aligned}
$$

and for $b_{0}=0$,

$$
C=\left(3 a_{0}^{2}+\frac{3}{2} d_{0}^{2} \cos 2 \delta_{0}+\frac{9}{2} a_{0}^{2} \cos 2 \alpha_{0}\right) T_{1}+c_{0}
$$

Equations (4.141), (4.147), (4.148), (4.153), (4.154), (4.169) and (4.170) are solutions to the conditions found in the previous sections which were found in order that $\Delta \hat{r}_{1}, \Delta \hat{\theta}_{1}$ and $\Delta \hat{\phi}_{1}$ were free of secular terms. The result found for the variable $c$ takes on two forms, depending on the value of $b_{0}$. It is unfortunate that the form of the solution depends on the initial conditions, but this does not present a problem of any kind. The next section will use the results obtained in (4.141), (4.147), (4.148), (4.153), (4.154), (4.162) and (4.164) along with (4.95), (4.96) and (4.101) and construct the solution in terms of only real variables, as well as to substitute back the value $\epsilon=1$ and simplify the resulting expression.

\subsubsection{Overall Solution}

The method of multiple scales has produced the approximate solution of equations (4.10), (4.11) and (4.12) accurate to order $\epsilon$ in the form

$$
\begin{aligned}
\Delta \hat{r}(\tau) & =\Delta \hat{r}_{0}\left(T_{0}, T_{1}\right)+\epsilon \Delta \hat{r}_{1}\left(T_{0}, T_{1}\right) \\
\Delta \hat{\theta}(\tau) & =\Delta \hat{\theta}_{0}\left(T_{0}, T_{1}\right)+\epsilon \Delta \hat{\theta}_{1}\left(T_{0}, T_{1}\right) \\
\Delta \hat{\phi}(\tau) & =\Delta \hat{\phi}_{0}\left(T_{0}, T_{1}\right)+\epsilon \Delta \hat{\phi}_{1}\left(T_{0}, T_{1}\right)
\end{aligned}
$$

In review, the results were

$$
\begin{aligned}
\Delta \hat{r}_{0} & =A \mathrm{e}^{i T_{0}}+\bar{A} \mathrm{e}^{-i T_{0}}+B \\
\Delta \hat{\theta}_{0} & =2 i A \mathrm{e}^{i T_{0}}-2 i \bar{A} \mathrm{e}^{-i T_{0}}-\frac{3}{2} B T_{0}+C \\
\Delta \hat{\phi}_{0} & =D \mathrm{e}^{i T_{0}}+\bar{D} \mathrm{e}^{-i T_{0}} \\
\Delta \hat{r}_{1} & =\left(\frac{9}{4} A^{2}+\frac{1}{2} A B+\frac{1}{4} D^{2}+\frac{1}{2} \bar{A} B+\frac{1}{4} \bar{A}^{2}+\frac{1}{4} \bar{D}^{2}-\frac{i \Delta \hat{v}_{r_{10}}}{2}-\frac{\Delta \hat{\omega}_{\theta_{10}}}{4}\right) \mathrm{e}^{i T_{0}}+
\end{aligned}
$$




$$
\begin{aligned}
& +\left(\frac{9}{4} \bar{A}^{2}+\frac{1}{2} \bar{A} B+\frac{1}{4} \bar{D}^{2}+\frac{1}{2} A B+\frac{1}{4} A^{2}+\frac{1}{4} D^{2}+\frac{i \Delta \hat{v}_{r_{10}}}{2}-\frac{\Delta \hat{\omega}_{\theta_{10}}}{4}\right) \mathrm{e}^{-i T_{0}}- \\
& -A^{2} \mathrm{e}^{2 i T_{0}}-\bar{A}^{2} \mathrm{e}^{-2 i T_{0}}-\frac{3}{2} A^{2}-\frac{3}{2} \bar{A}^{2}-A B-\bar{A} B-\frac{1}{2} D^{2}-\frac{1}{2} \bar{D}^{2}+\frac{\Delta \hat{\omega}_{\theta_{10}}}{2} \\
\Delta \hat{\theta}_{1}= & \left(\frac{9}{2} A^{2}-A B+\frac{1}{2} D^{2}+\bar{A} B+\frac{1}{2} \bar{A}^{2}+\frac{1}{2} \bar{D}^{2}-i \Delta \hat{v}_{r_{10}}-\frac{\Delta \hat{\omega}_{\theta_{10}}}{2}\right) i \mathrm{e}^{i T_{0}}+ \\
& +\left(-\frac{9 \bar{A}^{2}}{2}+\bar{A} B-\frac{1}{2} \bar{D}^{2}-A B-\frac{1}{2} A^{2}-\frac{1}{2} D^{2}-i \Delta \hat{v}_{r_{10}}+\frac{\Delta \hat{\omega}_{\theta_{10}}}{2}\right) i \mathrm{e}^{-i T_{0}}+ \\
& +\left(-\frac{5}{2} A^{2}-\frac{1}{2} D^{2}\right) i \mathrm{e}^{2 i T_{0}}+\left(\frac{5}{2} \bar{A}^{2}+\frac{1}{2} \bar{D}^{2}\right) i \mathrm{e}^{-2 i T_{0}}+ \\
& +\left(-\frac{3}{2} A^{2}+\frac{3}{2} \bar{A}^{2}+2 A B-2 \bar{A} B+\frac{1}{2} D^{2}-\frac{1}{2} \bar{D}^{2}+2 i \Delta \hat{v}_{r_{10}}\right) i \\
\Delta \hat{\phi}_{1}= & \left(3 A D-\bar{A} D-A \bar{D}-\bar{A} \bar{D}-\frac{i \Delta \hat{\omega}_{\phi_{10}}}{2}\right) \mathrm{e}^{i T_{0}}+ \\
& +\left(3 \bar{A} \bar{D}-A \bar{D}-\bar{A} D-A D+\frac{i \Delta \hat{\omega}_{\phi_{10}}}{2}\right) \mathrm{e}^{-i T_{0}}- \\
& -2 A D \mathrm{e}^{2 i T_{0}}-2 \bar{A} \bar{D} \mathrm{e}^{-2 i T_{0}}+2 A \bar{D}+2 \bar{A} D
\end{aligned}
$$

The derivatives of equations (4.171), (4.172) and (4.173) to order $\epsilon$ are

$$
\begin{aligned}
& \Delta \hat{v}_{r}(\tau)=\frac{\partial \Delta \hat{r}_{0}}{\partial T_{0}}+\epsilon\left(\frac{\partial \Delta \hat{r}_{0}}{\partial T_{1}}+\frac{\partial \Delta \hat{r}_{1}}{\partial T_{0}}\right) \\
& \Delta \hat{\omega}_{\theta}(\tau)=\frac{\partial \Delta \hat{\theta}_{0}}{\partial T_{0}}+\epsilon\left(\frac{\partial \Delta \hat{\theta}_{0}}{\partial T_{1}}+\frac{\partial \Delta \hat{\theta}_{1}}{\partial T_{0}}\right) \\
& \Delta \hat{\omega}_{\phi}(\tau)=\frac{\partial \Delta \hat{\phi}_{0}}{\partial T_{0}}+\epsilon\left(\frac{\partial \Delta \hat{\phi}_{0}}{\partial T_{1}}+\frac{\partial \Delta \hat{\phi}_{1}}{\partial T_{0}}\right)
\end{aligned}
$$

Evaluating the partial derivatives leads to

$$
\begin{aligned}
\frac{\partial \Delta \hat{r}_{0}}{\partial T_{0}}= & i A \mathrm{e}^{i T_{0}}-i \bar{A} \mathrm{e}^{-i T_{0}} \\
\frac{\partial \Delta \hat{r}_{0}}{\partial T_{1}}= & \frac{\partial A}{\partial T_{1}} \mathrm{e}^{i T_{0}}+\frac{\partial \bar{A}}{\partial T_{1}} \mathrm{e}^{-i T_{0}}+\frac{\partial B}{\partial T_{1}} \\
= & -\frac{3}{2} i A B \mathrm{e}^{i T_{0}}+\frac{3}{2} i \bar{A} B \mathrm{e}^{-i T_{0}} \\
\frac{\partial \Delta \hat{r}_{1}}{\partial T_{0}}= & i\left(\frac{9}{4} A^{2}+\frac{1}{2} A B+\frac{1}{4} D^{2}+\frac{1}{2} \bar{A} B+\frac{1}{4} \bar{A}^{2}+\frac{1}{4} \bar{D}^{2}-\frac{i \Delta \hat{v}_{r_{10}}}{2}-\frac{\Delta \hat{\omega}_{\theta_{10}}}{4}\right) \mathrm{e}^{i T_{0}}- \\
& -i\left(\frac{9}{4} \bar{A}^{2}+\frac{1}{2} \bar{A} B+\frac{1}{4} \bar{D}^{2}+\frac{1}{2} A B+\frac{1}{4} A^{2}+\frac{1}{4} D^{2}+\frac{i \Delta \hat{v}_{r_{10}}}{2}-\frac{\Delta \hat{\omega}_{\theta_{10}}}{4}\right) \mathrm{e}^{-i T_{0}}- \\
& -2 i A^{2} \mathrm{e}^{2 i T_{0}}+2 i \bar{A}^{2} \mathrm{e}^{-2 i T_{0}} \\
\frac{\partial \Delta \hat{\theta}_{0}}{\partial T_{0}}= & -2 A \mathrm{e}^{i T_{0}}-2 \bar{A} \mathrm{e}^{-i T_{0}}-\frac{3}{2} B
\end{aligned}
$$




$$
\begin{aligned}
\frac{\partial \Delta \hat{\theta}_{0}}{\partial T_{1}}= & 2 i \frac{\partial A}{\partial T_{1}} \mathrm{e}^{i T_{0}}-2 i \frac{\partial \bar{A}}{\partial T_{1}} \mathrm{e}^{-i T_{0}}-\frac{3}{2} \frac{\partial B}{\partial T_{1}} T_{0}+\frac{\partial C}{\partial T_{1}} \\
= & 3 A B \mathrm{e}^{i T_{0}}+3 \bar{A} B \mathrm{e}^{-i T_{0}}+\frac{15}{8} B^{2}+3 A \bar{A}+ \\
& +\frac{3}{4}\left(D^{2}+\bar{D}^{2}\right)+\frac{9}{4}\left(A^{2}+\bar{A}^{2}\right)+\frac{3}{2}(A B+\bar{A} B)-\frac{3}{4} \Delta \hat{\omega}_{\theta_{10}} \\
\frac{\partial \Delta \hat{\theta}_{1}}{\partial T_{0}}= & -\left(\frac{9}{2} A^{2}-A B+\frac{1}{2} D^{2}+\bar{A} B+\frac{1}{2} \bar{A}^{2}+\frac{1}{2} \bar{D}^{2}-i \Delta \hat{v}_{r_{10}}-\frac{\Delta \hat{\omega}_{\theta_{10}}}{2}\right) \mathrm{e}^{i T_{0}}+ \\
& +\left(-\frac{9 \bar{A}^{2}}{2}+\bar{A} B-\frac{1}{2} \bar{D}^{2}-A B-\frac{1}{2} A^{2}-\frac{1}{2} D^{2}-i \Delta \hat{v}_{r_{10}}+\frac{\Delta \hat{\omega}_{\theta_{10}}}{2}\right) \mathrm{e}^{-i T_{0}}+ \\
& +\left(5 A^{2}+D^{2}\right) \mathrm{e}^{2 i T_{0}}+\left(5 \bar{A}^{2}+\bar{D}^{2}\right) \mathrm{e}^{-2 i T_{0}} \\
\frac{\partial \Delta \hat{\phi}_{0}}{\partial T_{0}}= & i D \mathrm{e}^{i T_{0}}-i \bar{D} \mathrm{e}^{-i T_{0}} \\
\frac{\partial \Delta \hat{\phi}_{0}}{\partial T_{1}}= & \frac{\partial D}{\partial T_{1}} \mathrm{e}^{i T_{0}}+\frac{\partial \bar{D}}{\partial T_{1}} \mathrm{e}^{-i T_{0}} \\
= & -\frac{3}{2} i D B \mathrm{e}^{i T_{0}}+\frac{3}{2} i \bar{D} B \mathrm{e}^{-i T_{0}} \\
& -i\left(3 \bar{A} \bar{D}-A \bar{D}-\bar{A} D-A D+\frac{i \Delta \hat{\omega}_{\phi_{10}}}{2}\right) \mathrm{e}^{-i T_{0}}- \\
\frac{\partial \Delta \hat{\phi}_{1}}{\partial T_{0}}= & i\left(3 A D-\bar{A} D-A \bar{D}-\bar{A} \bar{D}-\frac{i \Delta \hat{\omega}_{\phi_{10}}}{2}\right) \mathrm{e}^{i T_{0}}- \\
& -4 i \bar{A} \bar{D} \mathrm{e}^{-2 i T_{0}} \\
& -i \bar{A}_{0}+
\end{aligned}
$$

Substituting these results into equations (4.171), (4.172), (4.173), (4.180), (4.181) and (4.182) gives

$$
\begin{aligned}
\Delta \hat{r}(\tau)= & A \mathrm{e}^{i T_{0}}+\bar{A} \mathrm{e}^{-i T_{0}}+B+ \\
& +\epsilon\left[\left(\frac{9}{4} A^{2}+\frac{1}{2} A B+\frac{1}{4} D^{2}+\frac{1}{2} \bar{A} B+\frac{1}{4} \bar{A}^{2}+\frac{1}{4} \bar{D}^{2}-\frac{i \Delta \hat{v}_{r_{10}}}{2}-\frac{\Delta \hat{\omega}_{\theta_{10}}}{4}\right) \mathrm{e}^{i T_{0}}+\right. \\
& +\left(\frac{9}{4} \bar{A}^{2}+\frac{1}{2} \bar{A} B+\frac{1}{4} \bar{D}^{2}+\frac{1}{2} A B+\frac{1}{4} A^{2}+\frac{1}{4} D^{2}+\frac{i \Delta \hat{v}_{r_{10}}}{2}-\frac{\Delta \hat{\omega}_{\theta_{10}}}{4}\right) \mathrm{e}^{-i T_{0}}- \\
& \left.-A^{2} \mathrm{e}^{2 i T_{0}}-\bar{A}^{2} \mathrm{e}^{-2 i T_{0}}-\frac{3}{2} A^{2}-\frac{3}{2} \bar{A}^{2}-A B-\bar{A} B-\frac{1}{2} D^{2}-\frac{1}{2} \bar{D}^{2}+\frac{\Delta \hat{\omega}_{\theta_{10}}}{2}\right] \\
\Delta \hat{\theta}(\tau)= & 2 i A \mathrm{e}^{i T_{0}}-2 i \bar{A} \mathrm{e}^{-i T_{0}}-\frac{3}{2} B T_{0}+C+ \\
& +\epsilon\left[\left(\frac{9}{2} A^{2}-A B+\frac{1}{2} D^{2}+\bar{A} B+\frac{1}{2} \bar{A}^{2}+\frac{1}{2} \bar{D}^{2}-i \Delta \hat{v}_{r_{10}}-\frac{\Delta \hat{\omega}_{\theta_{10}}}{2}\right) i \mathrm{e}^{i T_{0}}+\right. \\
& +\left(-\frac{9 \bar{A}^{2}}{2}+\bar{A} B-\frac{1}{2} \bar{D}^{2}-A B-\frac{1}{2} A^{2}-\frac{1}{2} D^{2}-i \Delta \hat{v}_{r_{10}}+\frac{\Delta \hat{\omega}_{\theta_{10}}}{2}\right) i \mathrm{e}^{-i T_{0}}+
\end{aligned}
$$




$$
\begin{aligned}
& +\left(-\frac{5}{2} A^{2}-\frac{1}{2} D^{2}\right) i \mathrm{e}^{2 i T_{0}}+\left(\frac{5}{2} \bar{A}^{2}+\frac{1}{2} \bar{D}^{2}\right) i \mathrm{e}^{-2 i T_{0}}+ \\
& \left.+\left(-\frac{3}{2} A^{2}+\frac{3}{2} \bar{A}^{2}+2 A B-2 \bar{A} B+\frac{1}{2} D^{2}-\frac{1}{2} \bar{D}^{2}+2 i \Delta \hat{v}_{r_{10}}\right) i\right] \\
& \Delta \hat{\phi}(\tau)=D \mathrm{e}^{i T_{0}}+\bar{D} \mathrm{e}^{-i T_{0}}+\epsilon\left[\left(3 A D-\bar{A} D-A \bar{D}-\bar{A} \bar{D}-\frac{i \Delta \hat{\omega}_{\phi_{10}}}{2}\right) \mathrm{e}^{i T_{0}}+\right. \\
& +\left(3 \bar{A} \bar{D}-A \bar{D}-\bar{A} D-A D+\frac{i \Delta \hat{\omega}_{\phi_{10}}}{2}\right) \mathrm{e}^{-i T_{0}}- \\
& \left.-2 A D \mathrm{e}^{2 i T_{0}}-2 \bar{A} \bar{D} \mathrm{e}^{-2 i T_{0}}+2 A \bar{D}+2 \bar{A} D\right] \\
& \Delta \hat{v}_{r}(\tau)=i A \mathrm{e}^{i T_{0}}-i \bar{A} \mathrm{e}^{-i T_{0}}+ \\
& +\epsilon\left[i\left(\frac{9}{4} A^{2}-A B+\frac{1}{4} D^{2}+\frac{1}{2} \bar{A} B+\frac{1}{4} \bar{A}^{2}+\frac{1}{4} \bar{D}^{2}-\frac{i \Delta \hat{v}_{r_{10}}}{2}-\frac{\Delta \hat{\omega}_{\theta_{10}}}{4}\right) \mathrm{e}^{i T_{0}}-\right. \\
& -i\left(\frac{9}{4} \bar{A}^{2}-\bar{A} B+\frac{1}{4} \bar{D}^{2}+\frac{1}{2} A B+\frac{1}{4} A^{2}+\frac{1}{4} D^{2}+\frac{i \Delta \hat{v}_{r_{10}}}{2}-\frac{\Delta \hat{\omega}_{\theta_{10}}}{4}\right) \mathrm{e}^{-i T_{0}}- \\
& \left.-2 i A^{2} \mathrm{e}^{2 i T_{0}}+2 i \bar{A}^{2} \mathrm{e}^{-2 i T_{0}}\right] \\
& \Delta \hat{\omega}_{\theta}(\tau)=-2 A \mathrm{e}^{i T_{0}}-2 \bar{A} \mathrm{e}^{-i T_{0}}-\frac{3}{2} B+ \\
& +\epsilon\left[-\left(\frac{9}{2} A^{2}-4 A B+\frac{1}{2} D^{2}+\bar{A} B+\frac{1}{2} \bar{A}^{2}+\frac{1}{2} \bar{D}^{2}-i \Delta \hat{v}_{r_{10}}-\frac{\Delta \hat{\omega}_{\theta_{10}}}{2}\right) \mathrm{e}^{i T_{0}}+\right. \\
& +\left(-\frac{9 \bar{A}^{2}}{2}+4 \bar{A} B-\frac{1}{2} \bar{D}^{2}-A B-\frac{1}{2} A^{2}-\frac{1}{2} D^{2}-i \Delta \hat{v}_{r_{10}}+\frac{\Delta \hat{\omega}_{\theta_{10}}}{2}\right) \mathrm{e}^{-i T_{0}}+ \\
& \left(5 A^{2}+D^{2}\right) \mathrm{e}^{2 i T_{0}}+\left(5 \bar{A}^{2}+\bar{D}^{2}\right) \mathrm{e}^{-2 i T_{0}}+\frac{15}{8} B^{2}+3 A \bar{A}+ \\
& \left.+\frac{3}{4}\left(D^{2}+\bar{D}^{2}\right)+\frac{9}{4}\left(A^{2}+\bar{A}^{2}\right)+\frac{3}{2}\left(A B+\bar{A} B-\frac{3}{4} \Delta \hat{\omega}_{\theta_{10}}\right)\right] \\
& \Delta \hat{\omega}_{\phi}(\tau)=i D \mathrm{e}^{i T_{0}}-i \bar{D} \mathrm{e}^{-i T_{0}}+\epsilon\left[i\left(3 A D-\bar{A} D-A \bar{D}-\bar{A} \bar{D}-\frac{3}{2} D B-\frac{i \Delta \hat{\omega}_{\phi_{10}}}{2}\right) \mathrm{e}^{i T_{0}}-\right. \\
& -i\left(3 \bar{A} \bar{D}-A \bar{D}-\bar{A} D-A D+\frac{3}{2} \bar{D} B+\frac{i \Delta \hat{\omega}_{\phi_{10}}}{2}\right) \mathrm{e}^{-i T_{0}}- \\
& \left.-4 i A D \mathrm{e}^{2 i T_{0}}+4 i \bar{A} \bar{D} \mathrm{e}^{-2 i T_{0}}\right]
\end{aligned}
$$

Where $A, B$ and $D$ are given by

$$
\begin{aligned}
A & =\frac{1}{2} a_{0} \mathrm{e}^{i\left(\alpha_{0}-\frac{3}{2} b_{0}\right) T_{1}} \\
B & =b_{0} \\
D & =\frac{1}{2} d_{0} \mathrm{e}^{i\left(\delta_{0}-\frac{3}{2} b_{0}\right) T_{1}}
\end{aligned}
$$

and $C$ takes two forms. If $b_{0}=0$, then

$$
C=\left(3 a_{0}^{2}+\frac{3}{2} d_{0}^{2} \cos 2 \delta_{0}+\frac{9}{2} a_{0}^{2} \cos 2 \alpha_{0}\right) T_{1}+c_{0}
$$


or, if $b_{0} \neq 0$, then

$$
\begin{aligned}
C= & \left(\frac{15}{2} b_{0}^{2}+3 a_{0}^{2}+\frac{27}{2} a_{0} b_{0} \cos \alpha_{0}+\frac{27}{8} \cos 2 \alpha_{0}+\frac{9}{8} \cos 2 \delta_{0}\right) T_{1}+ \\
& +\frac{1}{8} \frac{d_{0}^{2}}{b_{0}}\left[\sin \left(3 b_{0} T_{1}-2 \delta_{0}\right)+\sin 2 \delta_{0}\right]+\frac{3}{8} \frac{a_{0}^{2}}{b_{0}}\left[\sin \left(3 b_{0} T_{1}-2 \alpha_{0}\right)+\sin 2 \alpha_{0}\right]+ \\
& +a_{0}\left[\sin \left(\frac{3}{2} b_{0} T_{1}-\alpha_{0}\right)+\sin \alpha_{0}\right]+c_{0}
\end{aligned}
$$

Also, $\Delta \hat{v}_{r_{10}}, \Delta \hat{\omega}_{\theta_{10}}$ and $\Delta \hat{\omega}_{\phi_{10}}$ were found to be

$$
\begin{aligned}
\Delta \hat{v}_{r_{10}} & =-\frac{3}{2} a_{0} b_{0} \sin \alpha_{0} \\
\Delta \hat{\omega}_{\theta_{10}} & =-\frac{15}{2} b_{0}^{2}-3 a_{0}^{2}-18 a_{0} b_{0} \cos \alpha_{0}-\frac{9}{2} a_{0}^{2} \cos 2 \alpha_{0}-\frac{3}{2} d_{0}^{2} \cos 2 \delta_{0} \\
\Delta \hat{\omega}_{\phi_{10}} & =\frac{3}{2} d_{0} b_{0} \sin \delta_{0}
\end{aligned}
$$

Substituting these relations into equations $(4.192-4.197)$ and setting $\epsilon=1$, which also implies that $T_{1}=T_{0}=\tau$, gives the result

$$
\begin{aligned}
\Delta \hat{r}(\tau)= & a_{0} \cos \left[\left(1-\frac{3}{2} b_{0}\right) \tau+\alpha_{0}\right]+b_{0}+\frac{1}{4} \sin \left(\frac{\tau}{2}\right)\left[\operatorname { s i n } ( \frac { \tau } { 2 } ) \left(8 a_{0}^{2} \cos \left[2 \alpha_{0}+\left(1-3 b_{0}\right) \tau\right]-\right.\right. \\
& \left.-2 a_{0}^{2} \cos \left(2 \alpha_{0}-3 b_{0} \tau\right)-2 d_{0}^{2} \cos \left(2 \delta_{0}-3 b_{0} \tau\right)-18 a_{0}^{2} \cos 2 \alpha_{0}-6 d_{0}^{2} \cos 2 \delta_{0}-12 a_{0}^{2}\right)+ \\
& +2 a_{0} b_{0}\left[15 \sin \left(\alpha_{0}-\frac{1}{2} \tau\right)-21 \sin \left(\alpha_{0}+\frac{1}{2} \tau\right)-4 \sin \left(\frac{1}{2} \tau\right) \cos \left(\alpha_{0}-\frac{3}{2} b_{0} \tau\right)\right]- \\
& \left.-30 b_{0}^{2} \sin \left(\frac{\tau}{2}\right)\right] \\
\Delta \hat{\theta}(\tau)= & -2 a_{0} \sin \left[\left(1-\frac{3}{2} b_{0}\right) \tau+\alpha_{0}\right]-\frac{3}{2} b_{0} \tau+C(\tau)-\frac{1}{2} \sin \left(\frac{\tau}{2}\right)\left[9 a_{0}^{2} \cos \left(2 \alpha_{0}-\frac{1}{2} \tau\right)+\right. \\
& +3 d_{0}^{2} \cos \left(2 \delta_{0}-\frac{1}{2} \tau\right)+12 a_{0}^{2} \cos \left(\frac{\tau}{2}\right)+9 a_{0}^{2} \cos \left(2 \alpha_{0}+\frac{1}{2} \tau\right)+ \\
& +3 d_{0}^{2} \cos \left(2 \delta_{0}+\frac{1}{2} \tau\right)+4 a_{0}^{2} \cos \left[2 \alpha_{0}+\left(\frac{1}{2}-3 b_{0}\right) \tau\right]+ \\
& +a_{0}^{2} \cos \left[2 \alpha_{0}-\left(\frac{1}{2}+3 b_{0}\right) \tau\right]+d_{0}^{2} \cos \left[2 \delta_{0}-\left(\frac{1}{2}+3 b_{0}\right) \tau\right]- \\
& -5 a_{0}^{2} \cos \left[2 \alpha_{0}+\left(\frac{3}{2}-3 b_{0}\right) \tau\right]-d_{0}^{2} \cos \left[2 \delta_{0}+\left(\frac{3}{2}-3 b_{0}\right) \tau\right]+ \\
& +2 a_{0} b_{0}\left[15 \cos \left(\alpha_{0}-\frac{1}{2} \tau\right)+21 \cos \left(\alpha_{0}+\frac{1}{2} \tau\right)+4 \sin \left(\frac{\tau}{2}\right) \sin \left(\alpha_{0}-\frac{3}{2} b_{0} \tau\right)\right]+ \\
& \left.+30 b_{0}^{2} \cos \left(\frac{\tau}{2}\right)\right] \\
& \left.\left.+\cos \left(\alpha_{0}+\delta_{0}-3 b_{0} \tau\right)+2 \cos \left[\alpha_{0}+\delta_{0}+\left(1-3 b_{0}\right) \tau\right]\right]-3 b_{0} \sin \delta_{0} \sin \tau\right] \\
\Delta \hat{\phi}(\tau)= & d_{0} \cos \left[\left(1-\frac{3}{2} b_{0}\right) \tau+\delta_{0}\right]+\frac{1}{2} d_{0}\left[4 a _ { 0 } \operatorname { s i n } { } ^ { 2 } ( \frac { \tau } { 2 } ) \left[\cos \left(\alpha_{0}-\delta_{0}\right)+\right.\right.
\end{aligned}
$$




$$
\begin{aligned}
\Delta \hat{v}_{r}(\tau)= & -a_{0} \sin \left[\left(1-\frac{3}{2} b_{0}\right) \tau+\alpha_{0}\right]+\frac{1}{8}\left[9 a_{0}^{2} \sin \left(2 \alpha_{0}-\tau\right)+3 d_{0}^{2} \sin \left(2 \delta_{0}-\tau\right)-\right. \\
& -9 a_{0}^{2} \sin \left(2 \alpha_{0}+\tau\right)-3 d_{0}^{2} \sin \left(2 \delta_{0}+\tau\right)+a_{0}^{2} \sin \left[2 \alpha_{0}+\left(1+3 b_{0}\right) \tau\right]+ \\
& +d_{0}^{2} \sin \left[2 \delta_{0}-\left(1+3 b_{0}\right) \tau\right]-9 a_{0}^{2} \sin \left[2 \alpha_{0}+\left(1-3 b_{0}\right) \tau\right]-12 a_{0}^{2} \sin \tau- \\
& -d_{0}^{2} \sin \left[2 \delta_{0}+\left(1-3 b_{0}\right) \tau\right]+8 a_{0}^{2} \sin \left[2 \alpha_{0}+\left(2-3 b_{0}\right) \tau\right]+ \\
& +2 a_{0} b_{0}\left[15 \sin \left(\alpha_{0}-\tau\right)-21 \sin \left(\alpha_{0}+\tau\right)+2 \sin \left[\alpha_{0}-\left(1+\frac{3}{2} b_{0}\right) \tau\right]+\right. \\
& \left.\left.+4 \sin \left[\alpha_{0}+\left(1-\frac{3}{2} b_{0}\right) \tau\right]\right]-30 b_{0}^{2} \sin \tau\right] \\
\Delta \hat{\omega}_{\theta}(\tau)= & -2 a_{0} \cos \left[\left(1-\frac{3}{2} b_{0}\right) \tau+\alpha_{0}\right]-\frac{3}{2} b_{0}+\frac{1}{8}\left[24 a_{0}^{2}+27 a_{0}^{2} \cos 2 \alpha_{0}+9 d_{0}^{2} \cos 2 \delta_{0}-\right. \\
& -18 a_{0}^{2} \cos \left(2 \alpha_{0}-\tau\right)-6 d_{0}^{2} \cos \left(2 \delta_{0}-\tau\right)-24 a_{0}^{2} \cos \tau- \\
& -18 a_{0}^{2} \cos \left(2 \alpha_{0}+\tau\right)-6 d_{0}^{2} \cos \left(2 \delta_{0}+\tau\right)+9 a_{0}^{2} \cos \left(2 \alpha_{0}-3 b_{0} \tau\right)+ \\
& +3 d_{0}^{2} \cos \left(2 \delta_{0}-3 b_{0} \tau\right)-2 a_{0}^{2} \cos \left[2 \alpha_{0}-\left(1-3 b_{0}\right) \tau\right]-2 d_{0}^{2} \cos \left[2 \delta_{0}-\left(1+3 b_{0}\right) \tau\right]- \\
& -18 a_{0}^{2} \cos \left[2 \alpha_{0}+\left(1-3 b_{0}\right) \tau\right]-2 d_{0}^{2} \cos \left[2 \delta_{0}+\left(1-3 b_{0}\right) \tau\right]+ \\
& +20 a_{0}^{2} \cos \left[2 \alpha_{0}+\left(2-3 b_{0}\right) \tau\right]+4 d_{0}^{2} \cos \left[2 \delta_{0}+\left(2-3 b_{0}\right) \tau\right]+ \\
& +4 a_{0} b_{0}\left[27 \cos \alpha_{0}-15 \cos \left(\alpha_{0}-\tau\right)-21 \cos \left(\alpha_{0}+\tau\right)+3 \cos \left(\alpha_{0}-\frac{3}{2} b_{0} \tau\right)-\right. \\
& \left.\left.-2 \cos \left[\alpha_{0}-\left(1+\frac{3}{2} b_{0}\right) \tau\right]+8 \cos \left[\alpha_{0}+\left(1-\frac{3}{2} b_{0}\right) \tau\right]\right]-60 b_{0}^{2}(\cos \tau-1)\right] \\
& \left.+4 a_{0} \sin \left[\alpha_{0}+\delta_{0}+\left(2-3 b_{0}\right) \tau\right]-3 b_{0} \sin \delta_{0} \cos \tau+3 b_{0} \sin \left[\delta_{0}+\left(1-\frac{3}{2}\right) \tau\right]\right] \\
& -2 a_{0} \cos \left(\alpha_{0}+\delta_{0}-3 b_{0} \tau\right) \sin \tau-4 a_{0} \sin \left(\alpha_{0}+\delta_{0}-3 b_{0} \tau\right) \cos \tau+ \\
\Delta \hat{\omega}_{\phi}(\tau)= & d_{0} \sin \left[\left(1-\frac{3}{2} b_{0}\right) \tau+\delta_{0}\right]+\frac{1}{2} d_{0}\left[2 a_{0} \cos \left(\alpha_{0}-\delta_{0}\right) \sin \tau-\right. \\
& -110
\end{aligned}
$$

where

$$
C(\tau)=\left(3 a_{0}^{2}+\frac{3}{2} d_{0}^{2} \cos 2 \delta_{0}+\frac{9}{2} a_{0}^{2} \cos 2 \alpha_{0}\right) \tau+c_{0}
$$

if $b_{0}=0$ and

$$
\begin{aligned}
C(\tau)= & \left(\frac{15}{2} b_{0}^{2}+3 a_{0}^{2}+\frac{27}{2} a_{0} b_{0} \cos \alpha_{0}+\frac{27}{8} \cos 2 \alpha_{0}+\frac{9}{8} \cos 2 \delta_{0}\right) \tau+ \\
& +\frac{1}{8} \frac{d_{0}^{2}}{b_{0}}\left[\sin \left(3 b_{0} \tau-2 \delta_{0}\right)+\sin 2 \delta_{0}\right]+\frac{3}{8} \frac{a_{0}^{2}}{b_{0}}\left[\sin \left(3 b_{0} \tau-2 \alpha_{0}\right)+\sin 2 \alpha_{0}\right]+ \\
& +a_{0}\left[\sin \left(\frac{3}{2} b_{0} \tau-\alpha_{0}\right)+\sin \alpha_{0}\right]+c_{0}
\end{aligned}
$$

if $b_{0} \neq 0$. The parameters $a_{0}, \alpha_{0}, b_{0}, c_{0}, d_{0}$ and $\delta_{0}$ are calculated from

$$
\begin{aligned}
a_{0}^{2} & =\left(3 \Delta \hat{r}_{00}+2 \Delta \hat{\omega}_{\theta_{00}}\right)^{2}+\Delta \hat{v}_{r_{00}}^{2} \\
d_{0}^{2} & =\Delta \hat{\phi}_{00}^{2}+\Delta \hat{\omega}_{\phi_{00}}^{2} \\
\sin \alpha_{0} & =-\frac{\Delta \hat{v}_{r_{00}}}{a}
\end{aligned}
$$




$$
\begin{aligned}
\cos \alpha_{0} & =-\frac{3 \Delta \hat{r}_{00}+2 \Delta \hat{\omega}_{\theta_{00}}}{a} \\
\sin \delta_{0} & =-\frac{\Delta \hat{\omega}_{\phi_{00}}}{d} \\
\cos \delta_{0} & =\frac{\Delta \hat{\phi}_{00}}{d} \\
b_{0} & =4 \Delta \hat{r}_{00}+2 \Delta \hat{\omega}_{\theta_{00}} \\
c_{0} & =\Delta \hat{\theta}_{00}-2 \Delta \hat{v}_{r_{00}}
\end{aligned}
$$

and the relative position and velocity vectors are given by

$$
\hat{\mathbf{R}}=[(1+\Delta \hat{r}) \cos \Delta \hat{\phi} \cos \Delta \hat{\theta}-1] \mathbf{e}_{r}+(1+\Delta \hat{r}) \cos \Delta \hat{\phi} \sin \Delta \hat{\theta} \mathbf{e}_{\theta}+(1+\Delta \hat{r}) \sin \Delta \hat{\phi} \mathbf{e}_{\phi}
$$

and

$$
\begin{aligned}
\hat{\mathbf{V}}= & {\left[\Delta \hat{v}_{r} \cos \Delta \hat{\phi} \cos \Delta \hat{\theta}-(1+\Delta \hat{r}) \Delta \hat{\omega}_{\phi} \sin \Delta \hat{\phi} \cos \Delta \hat{\theta}-\right.} \\
& \left.-(1+\Delta \hat{r}) \Delta \hat{\omega}_{\theta} \cos \Delta \hat{\phi} \sin \Delta \hat{\theta}-(1+\Delta \hat{r}) \cos \Delta \hat{\phi} \sin \Delta \hat{\theta}\right] \mathbf{e}_{r}+ \\
& +\left[\Delta \hat{v}_{r} \cos \Delta \hat{\phi} \sin \Delta \hat{\theta}-(1+\Delta \hat{r}) \Delta \hat{\omega}_{\phi} \sin \Delta \hat{\phi} \sin \Delta \hat{\theta}+\right. \\
& \left.+(1+\Delta \hat{r}) \Delta \hat{\omega}_{\theta} \cos \Delta \hat{\phi} \cos \Delta \hat{\theta}+(1+\Delta \hat{r}) \cos \Delta \hat{\phi} \cos \Delta \hat{\theta}-1\right] \mathbf{e}_{\theta}+ \\
& +\left[\Delta \hat{v}_{r} \sin \Delta \hat{\phi}+(1+\Delta \hat{r}) \Delta \hat{\omega}_{\phi} \cos \Delta \hat{\phi}\right] \mathbf{e}_{\phi}
\end{aligned}
$$

\subsection{Summary}

This chapter has given the solution of relative motion equations which contain quadratic nonlinearity. Using the linear solution as a starting point, the method of multiple scales was employed to obtain a uniform approximation to the solution of the nonlinear relative motion equations. The expansion produced here is valid for only one correction term to the linear solution, as secular terms cannot be eliminated when a second correction term (and its corresponding slow time scale, $T_{2}$ ) are introduced to the problem.

The next chapter compares the new second order relative motion equations that are derived in this chapter against the first order relative motion equations derived in chapter (3) to understand what improvements have been made. 


\section{Chapter 5}

\section{Results}

This chapter compares the new, nonlinear, relative motion equations found in (4.206) through (4.211) against the previous, linear, relative motions equations found in (3.81). A comparison is also made by comparing the approximate analytical solution of equation (4.10) through (4.12) against a numerical solution to understand how closely the analytical approximation follows the numerical solution. This comparison is made using the following procedure:

1. Specify initial conditions $\Delta \hat{r}(0), \Delta \hat{\theta}(0), \Delta \hat{\phi}(0), \Delta \hat{v}_{r}(0), \Delta \hat{\omega}_{\theta}(0)$ and $\Delta \hat{\omega}_{\phi}(0)$

2. Numerically integrate the full, nonlinear equations of motion given in (2.14), (2.15) and (2.16)

3. Numerically integrate the quadratic equations of relative motion given in (4.10), (4.11) and (4.12)

4. Calculate the analytical solution of the quadratic equations found in (4.206) through (4.211)

5. Calculate the solution of the linear relative motion equations found in (3.81)

6. For each of the above cases, calculate the relative position and velocity vectors using equations (3.109) and (3.113)

7. Define a state error to be the approximate solution (found by either an analytical technique or numerical integration of approximate equations) minus the numerical integration of the full nonlinear equations of motion

8. Define relative position and velocity errors to the relative position and velocity computed from an approximation minus the numerical integration of the full nonlinear equations of motion

9. Plot the state errors and magnitude of relative position and velocity errors versus time for two periods of the reference orbit 
The results of this comparison are in appendix (C). The first line of each plot shows what the initial conditions of the problem, with everything not mentioned assumed to be zero. The first series of plots, (C.1) through (C.15), shows the response due to one nonzero initial state at a time. Figures (C.16) through (C.33) shows the results from a combination of in-plane and out-of-plane initial conditions. The value for each nonzero initial condition is 0.001, which, for the case of a radial separation, would be roughly 7 kilometers for a low Earth orbit.

A significant improvement has been made with the new nonlinear relative motion equations over the linear relative motion equations. For most cases, the linear solution begins to depart from the numerical integration after roughly $\frac{1}{4}$ of the period of the reference orbit, while the nonlinear equations show a much smaller variation - in many cases the solution appears exact on the scale shown (in all cases this scaling depends on the error of the linear equations). The approximate analytical solution of equations (4.10), (4.11) and (4.12) also follows the numerical approximation quite well, and only small deviations are visible toward the end of the time scale shown.

Note that for only in-plane initial conditions there is no out-of-plane motion, therefore the error for $\Delta \hat{\phi}$ and $\Delta \hat{\omega}_{\phi}$ in each of these cases is identically zero. Also note that in many cases the error amplitude is growing in time, such as that shown in (C.1). It should be made clear that this amplitude results from a difference in the frequency of oscillation, and remains bounded in time. This is also true for the plots of the magnitude of relative position and velocity - since the solutions contain no false secular terms these errors must be bounded.

For cases of just out-of-plane initial separations, such as shown in figure (C.13), the new solution captures the in-plane coupling quite well, but the out-of-plane motion reverts back to the linear solution. Note, however, that the scale of the out-of-plane error is quite a bit smaller than the scale of the in-plane error, so this approximation is still relatively good. 


\section{Chapter 6}

\section{Conclusions}

The purpose of this thesis was to develop a solution of the relative motion problem which results from a higher order expansion of the equations of motion (2.1). A further requirement was that the solution be uniform in the sense described in chapter (2). This solution was developed using the method of multiple scales, which made use of the solution to the first order expansion of the equations of motion. The new solution has several advantages when compared with the linear solution:

- Improved accuracy

- Re-coupling of motion in the plane and out of the plane of the reference orbit

- Better estimate of the drift rate between orbits of differing periods

The new solution does have the disadvantage that it is rather bulky and contains many terms, although this ceases to be a problem if the solution is implemented on a computer.

As always, there is more work that could be done on this problem. First, one may wish to re-formulate the problem slightly by expanding the equations of motion about an slightly eccentric orbit in a manner similar to the work of [3] or [24]. This would lead to a system of differential equations that is both nonlinear as well as nonautonomous, therefore it may not be possible to obtain a uniform expansion using the method of multiple scales. The only way to know for sure is to try it.

Another possibility is to consider gravitational perturbations that are caused by a nonspherical primary body. The first step would be to consider the so-called $J_{2}$ perturbation - the first in a series expansion of the primary body's gravitational potential. The $J_{2}$ perturbation leads to what is called "nodal regression", where the plane of the orbit regresses around the primary body (see reference [25]). Therefore, one would expect to see secular terms arise in the solution for the out-of-plane motion. This secular motion can be subtracted out of the solution using other analytical results for the regression rate in order to yield motion relative to an orbit that is also regressing in response to the gravitational perturbation. 
The new solution may also be applied to the applications discussed in chapter (1). A classic problem would be the optimal two-impulse rendezvous maneuver studied in several of the references. A comparison can also be made here between the linear solution and the nonlinear solution by examining miss distances and the total fuel requirements among other things.

Another use for the new solution is in the area of satellite formations. There are several areas for further study in this area. For a given formation geometry, a higher order estimate of the drift rate between the satellites is found in the new solution. This drift rate may be used to determine the amount of fuel required for station keeping, for example. The reverse of this problem is to use the new estimate of the drift rate to design the formation geometry such that this rate is zero, so that (at second order) the satellites would remain in formation. This would require a numerical study to understand the unmodeled third order effects and other perturbations.

An unresolved problem with this solution is the presence of the constant offset term in the equation for $\Delta \hat{\phi}$. The constant term does not satisfy conservation of angular momentum, although it seems to appear on every occasion that nonlinear terms are kept in the expansion of equation (2.1). Further work should also be focused on removing this constant from the solution. 


\section{Appendix A}

\section{Nonlinear Model}

The quadratic expansion of the radial equation was

$$
\begin{aligned}
\dot{v}_{r}= & {\left[\dot{v}_{r}\right]_{r e f}+\Delta r\left[\frac{\partial \dot{v}_{r}}{\partial r}\right]_{r e f}+\Delta \theta\left[\frac{\partial \dot{v}_{r}}{\partial \theta}\right]_{r e f}+\Delta \phi\left[\frac{\partial \dot{v}_{r}}{\partial \phi}\right]_{r e f}+\Delta v_{r}\left[\frac{\partial \dot{v}_{r}}{\partial v_{r}}\right]_{r e f}+} \\
& +\Delta \omega_{\theta}\left[\frac{\partial \dot{v}_{r}}{\partial \omega_{\theta}}\right]_{r e f}+\Delta \omega_{\phi}\left[\frac{\partial \dot{v}_{r}}{\partial \omega_{\phi}}\right]_{r e f}+\frac{1}{2} \Delta r^{2}\left[\frac{\partial^{2} \dot{v}_{r}}{\partial r^{2}}\right]_{r e f}+\Delta \theta \Delta r\left[\frac{\partial^{2} \dot{v}_{r}}{\partial \theta \partial r}\right]_{r e f}+ \\
& +\Delta \phi \Delta r\left[\frac{\partial^{2} \dot{v}_{r}}{\partial \phi \partial r}\right]_{r e f}+\Delta v_{r} \Delta r\left[\frac{\partial^{2} \dot{v}_{r}}{\partial v_{r} \partial r}\right]_{r e f}+\Delta \omega_{\theta} \Delta r\left[\frac{\partial^{2} \dot{v}_{r}}{\partial \omega_{\theta} \partial r}\right]_{r e f}+ \\
& +\Delta \omega_{\phi} \Delta r\left[\frac{\partial^{2} \dot{v}_{r}}{\partial \omega_{\phi} \partial r}\right]_{r e f}+\frac{1}{2} \Delta \theta^{2}\left[\frac{\partial^{2} \dot{v}_{r}}{\partial \theta^{2}}\right]_{r e f}+\Delta \phi \Delta \theta\left[\frac{\partial^{2} \dot{v}_{r}}{\partial \phi \partial \theta}\right]_{r e f}+ \\
& +\Delta v_{r} \Delta \theta\left[\frac{\partial^{2} \dot{v}_{r}}{\partial v_{r} \partial \theta}\right]_{r e f}+\Delta \omega_{\theta} \Delta \theta\left[\frac{\partial^{2} \dot{v}_{r}}{\partial \omega_{\theta} \partial \theta}\right]_{r e f}+\Delta \omega_{\phi} \Delta \theta\left[\frac{\partial^{2} \dot{v}_{r}}{\partial \omega_{\phi} \partial \theta}\right]_{r e f}+ \\
& +\frac{1}{2} \Delta \phi^{2}\left[\frac{\partial^{2} \dot{v}_{r}}{\partial \phi^{2}}\right]_{r e f}+\Delta v_{r} \Delta \phi\left[\frac{\partial^{2} \dot{v}_{r}}{\partial v_{r} \partial \phi}\right]_{r e f}+\Delta \omega_{\theta} \Delta \phi\left[\frac{\partial^{2} \dot{v}_{r}}{\partial \omega_{\theta} \partial \phi}\right]_{r e f}+ \\
& +\Delta \omega_{\phi} \Delta \phi\left[\frac{\partial^{2} \dot{v}_{r}}{\partial \omega_{\phi} \partial \phi}\right]_{r e f}+\frac{1}{2} \Delta v_{r}^{2}\left[\frac{\partial^{2} \dot{v}_{r}}{\partial v_{r}^{2}}\right]_{r e f}+\Delta \omega_{\theta} \Delta v_{r}\left[\frac{\partial^{2} \dot{v}_{r}}{\partial \omega_{\theta} \partial v_{r}}\right]_{r e f}+ \\
& +\Delta \omega_{\phi} \Delta v_{r}\left[\frac{\partial^{2} \dot{v}_{r}}{\partial \omega_{\phi} \partial v_{r}}\right]_{r e f}+\frac{\partial^{2}}{2} \Delta \omega_{\theta}^{2}\left[\frac{\partial^{2} \dot{v}_{r}}{\partial \omega_{\theta}^{2}}\right]_{r e f}+\Delta \omega_{\theta} \Delta \omega_{\phi}\left[\frac{\partial^{2} \dot{v}_{r}}{\partial \omega_{\phi} \partial \omega_{\theta}}\right]_{r e f}+ \\
& +\frac{1}{2} \Delta \omega_{\phi}^{2}\left[\frac{\partial^{2} \dot{v}_{r}}{\partial \omega_{\phi}^{2}}\right]_{r e f} \\
& +
\end{aligned}
$$

Evaluating the coefficients of the nonlinear terms (keeping in mind that all derivatives with respect to $\theta$ will be zero since it is a cyclic variable) leads to

$$
\left[\frac{\partial^{2} \dot{v}_{r}}{\partial r^{2}}\right]_{r e f}=\left[-\frac{6 \mu}{r^{4}}\right]_{r e f}
$$




$$
\begin{aligned}
& =-\frac{6 \omega_{0}^{2}}{r_{0}} \\
& {\left[\frac{\partial^{2} \dot{v}_{r}}{\partial \phi \partial r}\right]_{r e f}=\left[-2 \omega_{\theta}^{2} \cos \phi \sin \phi\right]_{r e f}} \\
& =0 \\
& {\left[\frac{\partial^{2} \dot{v}_{r}}{\partial v_{r} \partial r}\right]_{r e f}=0} \\
& {\left[\frac{\partial^{2} \dot{v}_{r}}{\partial \omega_{\theta} \partial r}\right]_{r e f}=\left[2 \omega_{\theta} \cos ^{2} \phi\right]_{r e f}} \\
& =2 \omega_{0} \\
& {\left[\frac{\partial^{2} \dot{v}_{r}}{\partial \omega_{\phi} \partial r}\right]_{r e f}=\left[2 \omega_{\phi}\right]_{r e f}} \\
& =0 \\
& {\left[\frac{\partial^{2} \dot{v}_{r}}{\partial \phi^{2}}\right]_{r e f}=\left[2 r \omega_{\theta}^{2} \sin ^{2} \phi-2 r \omega_{\theta}^{2} \cos ^{2} \phi\right]_{r e f}} \\
& =-2 r_{0} \omega_{0}^{2} \\
& {\left[\frac{\partial^{2} \dot{v}_{r}}{\partial v_{r} \partial \phi}\right]_{r e f}=0} \\
& {\left[\frac{\partial^{2} \dot{v}_{r}}{\partial \omega_{\theta} \partial \phi}\right]_{r e f}=\left[-4 r \omega_{\theta} \cos \phi \sin \phi\right]_{r e f}} \\
& =0 \\
& {\left[\frac{\partial^{2} \dot{v}_{r}}{\partial \omega_{\phi} \partial \phi}\right]_{r e f}=0} \\
& {\left[\frac{\partial^{2} \dot{v}_{r}}{\partial v_{r}^{2}}\right]_{r e f}=0} \\
& {\left[\frac{\partial^{2} \dot{v}_{r}}{\partial \omega_{\theta} \partial v_{r}}\right]_{r e f}=0} \\
& {\left[\frac{\partial^{2} \dot{v}_{r}}{\partial \omega_{\phi} \partial v_{r}}\right]_{r e f}=0}
\end{aligned}
$$




$$
\begin{aligned}
{\left[\frac{\partial^{2} \dot{v}_{r}}{\partial \omega_{\theta}^{2}}\right]_{r e f} } & =\left[2 r \cos ^{2} \phi\right]_{r e f} \\
& =2 r_{0} \\
{\left[\frac{\partial^{2} \dot{v}_{r}}{\partial \omega_{\phi} \partial \omega_{\theta}}\right]_{r e f} } & =0 \\
{\left[\frac{\partial^{2} \dot{v}_{r}}{\partial \omega_{\phi}^{2}}\right]_{r e f} } & =[2 r]_{r e f} \\
& =2 r_{0}
\end{aligned}
$$

which results in

$$
\begin{aligned}
\Delta \dot{v}_{r}= & 3 \omega_{0}^{2} \Delta r+2 r_{0} \omega_{0} \Delta \omega_{\theta}-\frac{3 \omega_{0}^{2}}{r_{0}} \Delta r^{2}+2 \omega_{0} \Delta r \Delta \omega_{\theta}- \\
& -r_{0} \omega_{0}^{2} \Delta \phi^{2}+r_{0} \Delta \omega_{\theta}^{2}+r_{0} \Delta \omega_{\phi}^{2}
\end{aligned}
$$

The quadratic expansion of the transverse equation was

$$
\begin{aligned}
\dot{\omega}_{\theta}= & {\left[\dot{\omega}_{\theta}\right]_{r e f}+\Delta r\left[\frac{\partial \dot{\omega}_{\theta}}{\partial r}\right]_{r e f}+\Delta \theta\left[\frac{\partial \dot{\omega}_{\theta}}{\partial \theta}\right]_{r e f}+\Delta \phi\left[\frac{\partial \dot{\omega}_{\theta}}{\partial \phi}\right]_{r e f}+\Delta v_{r}\left[\frac{\partial \dot{\omega}_{\theta}}{\partial v_{r}}\right]_{r e f}+} \\
& +\Delta \omega_{\theta}\left[\frac{\partial \dot{\omega}_{\theta}}{\partial \omega_{\theta}}\right]_{r e f}+\Delta \omega_{\phi}\left[\frac{\partial \dot{\omega}_{\theta}}{\partial \omega_{\phi}}\right]_{r e f}+\frac{1}{2} \Delta r^{2}\left[\frac{\partial^{2} \dot{\omega}_{\theta}}{\partial r^{2}}\right]_{r e f}+\Delta \theta \Delta r\left[\frac{\partial^{2} \dot{\omega}_{\theta}}{\partial \theta \partial r}\right]_{r e f}+ \\
& +\Delta \phi \Delta r\left[\frac{\partial^{2} \dot{\omega}_{\theta}}{\partial \phi \partial r}\right]_{r e f}+\Delta v_{r} \Delta r\left[\frac{\partial^{2} \dot{\omega}_{\theta}}{\partial v_{r} \partial r}\right]_{r e f}+\Delta \omega_{\theta} \Delta r\left[\frac{\partial^{2} \dot{\omega}_{\theta}}{\partial \omega_{\theta} \partial r}\right]_{r e f}+ \\
& +\Delta \omega_{\phi} \Delta r\left[\frac{\partial^{2} \dot{\omega}_{\theta}}{\partial \omega_{\phi} \partial r}\right]_{r e f}+\frac{1}{2} \Delta \theta^{2}\left[\frac{\partial^{2} \dot{\omega}_{\theta}}{\partial \theta^{2}}\right]_{r e f}+\Delta \phi \Delta \theta\left[\frac{\partial^{2} \dot{\omega}_{\theta}}{\partial \phi \partial \theta}\right]_{r e f}+ \\
& +\Delta v_{r} \Delta \theta\left[\frac{\partial^{2} \dot{\omega}_{\theta}}{\partial v_{r} \partial \theta}\right]_{r e f}+\Delta \omega_{\theta} \Delta \theta\left[\frac{\partial^{2} \dot{\omega}_{\theta}}{\partial \omega_{\theta} \partial \theta}\right]_{r e f}+\Delta \omega_{\phi} \Delta \theta\left[\frac{\partial^{2} \dot{\omega}_{\theta}}{\partial \omega_{\phi} \partial \theta}\right]_{r e f}+ \\
& +\frac{1}{2} \Delta \phi^{2}\left[\frac{\partial^{2} \dot{\omega}_{\theta}}{\partial \phi^{2}}\right]_{r e f}+\Delta v_{r} \Delta \phi\left[\frac{\partial^{2} \dot{\omega}_{\theta}}{\partial v_{r} \partial \phi}\right]_{r e f}+\Delta \omega_{\theta} \Delta \phi\left[\frac{\partial^{2} \dot{\omega}_{\theta}}{\partial \omega_{\theta} \partial \phi}\right]_{r e f}+ \\
& +\Delta \omega_{\phi} \Delta \phi\left[\frac{\partial^{2} \dot{\omega}_{\theta}}{\partial \omega_{\phi} \partial \phi}\right]_{r e f}+\frac{1}{2} \Delta v_{r}^{2}\left[\frac{\partial^{2} \dot{\omega}_{\theta}}{\partial v_{r}^{2}}\right]_{r e f}+\Delta \omega_{\theta} \Delta v_{r}\left[\frac{\partial^{2} \dot{\omega}_{\theta}}{\partial \omega_{\theta} \partial v_{r}}\right]_{r e f}+ \\
& +\Delta \omega_{\phi} \Delta v_{r}\left[\frac{\partial^{2} \dot{\omega}_{\theta}}{\partial \omega_{\phi} \partial v_{r}}\right]_{r e f}+\frac{1}{2} \Delta \omega_{\theta}^{2}\left[\frac{\partial^{2} \dot{\omega}_{\theta}}{\partial \omega_{\theta}^{2}}\right]_{r e f}+\Delta \omega_{\theta} \Delta \omega_{\phi}\left[\frac{\partial^{2} \dot{\omega}_{\theta}}{\partial \omega_{\phi} \partial \omega_{\theta}}\right]_{r e f}+ \\
& +\frac{1}{2} \Delta \omega_{\phi}^{2}\left[\frac{\partial^{2} \dot{\omega}_{\theta}}{\partial \omega_{\phi}^{2}}\right]_{r e f}
\end{aligned}
$$

where the coefficients of the nonlinear terms are

$$
\left[\frac{\partial^{2} \dot{\omega}_{\theta}}{\partial r^{2}}\right]_{r e f}=\left[-\frac{4 v_{r} \omega_{\theta}}{r^{3}}\right]_{r e f}
$$




$$
=0
$$

$\left[\frac{\partial^{2} \dot{\omega}_{\theta}}{\partial \phi \partial r}\right]_{r e f}=0$

$$
\begin{aligned}
{\left[\frac{\partial^{2} \dot{\omega}_{\theta}}{\partial v_{r} \partial r}\right]_{r e f} } & =\left[\frac{2 \omega_{\theta}}{r^{2}}\right]_{r e f} \\
& =\frac{2 \omega_{0}}{r_{0}}
\end{aligned}
$$

$$
\begin{aligned}
{\left[\frac{\partial^{2} \dot{\omega}_{\theta}}{\partial \omega_{\theta} \partial r}\right]_{r e f} } & =\left[\frac{2 v_{r}}{r^{2}}\right]_{r e f} \\
& =0
\end{aligned}
$$

$$
\left[\frac{\partial^{2} \dot{\omega}_{\theta}}{\partial \omega_{\phi} \partial r}\right]_{r e f}=0
$$

$$
\begin{aligned}
{\left[\frac{\partial^{2} \dot{\omega}_{\theta}}{\partial \phi^{2}}\right]_{r e f} } & =\left[4 \omega_{\phi} \omega_{\theta} \sec ^{2} \phi \tan \phi\right]_{r e f} \\
& =0
\end{aligned}
$$

$$
\left[\frac{\partial^{2} \dot{\omega}_{\theta}}{\partial v_{r} \partial \phi}\right]_{r e f}=0
$$

$$
\begin{aligned}
{\left[\frac{\partial^{2} \dot{\omega}_{\theta}}{\partial \omega_{\theta} \partial \phi}\right]_{r e f} } & =\left[2 \omega_{\phi} \sec ^{2} \phi\right]_{r e f} \\
& =0
\end{aligned}
$$

$$
\begin{aligned}
{\left[\frac{\partial^{2} \dot{\omega}_{\theta}}{\partial \omega_{\phi} \partial \phi}\right]_{r e f} } & =\left[2 \omega_{\theta}^{2} \sec ^{2} \phi\right]_{r e f} \\
& =2 \omega_{0}
\end{aligned}
$$

$$
\begin{aligned}
{\left[\frac{\partial^{2} \dot{\omega}_{\theta}}{\partial v_{r}^{2}}\right]_{r e f} } & =0 \\
{\left[\frac{\partial^{2} \dot{\omega}_{\theta}}{\partial \omega_{\theta} \partial v_{r}}\right]_{r e f} } & =\left[-\frac{2}{r}\right]_{r e f} \\
& =-\frac{2}{r_{0}} \\
{\left[\frac{\partial^{2} \dot{\omega}_{\theta}}{\partial \omega_{\phi} \partial v_{r}}\right]_{r e f} } & =0
\end{aligned}
$$




$$
\begin{aligned}
{\left[\frac{\partial^{2} \dot{\omega}_{\theta}}{\partial \omega_{\theta}^{2}}\right]_{r e f} } & =0 \\
{\left[\frac{\partial^{2} \dot{\omega}_{\theta}}{\partial \omega_{\phi} \partial \omega_{\theta}}\right]_{r e f} } & =[2 \tan \phi]_{r e f} \\
& =0 \\
{\left[\frac{\partial^{2} \dot{\omega}_{\theta}}{\partial \omega_{\phi}^{2}}\right]_{r e f} } & =0
\end{aligned}
$$

which results in

$$
\Delta \dot{\omega}_{\theta}=-\frac{2 \omega_{0}}{r_{0}} \Delta v_{r}+\frac{2 \omega_{0}}{r_{0}^{2}} \Delta v_{r} \Delta r+2 \omega_{0} \Delta \omega_{\phi} \Delta \phi-\frac{2}{r_{0}} \Delta \omega_{\theta} \Delta v_{r}
$$

The quadratic expansion of the out-of-plane equation was

$$
\begin{aligned}
\dot{\omega}_{\phi}= & {\left[\dot{\omega}_{\phi}\right]_{r e f}+\Delta r\left[\frac{\partial \dot{\omega}_{\phi}}{\partial r}\right]_{r e f}+\Delta \theta\left[\frac{\partial \dot{\omega}_{\phi}}{\partial \theta}\right]_{r e f}+\Delta \phi\left[\frac{\partial \dot{\omega}_{\phi}}{\partial \phi}\right]_{r e f}+\Delta v_{r}\left[\frac{\partial \dot{\omega}_{\phi}}{\partial v_{r}}\right]_{r e f}+} \\
& +\Delta \omega_{\theta}\left[\frac{\partial \dot{\omega}_{\phi}}{\partial \omega_{\theta}}\right]_{r e f}+\Delta \omega_{\phi}\left[\frac{\partial \dot{\omega}_{\phi}}{\partial \omega_{\phi}}\right]_{r e f}+\frac{1}{2} \Delta r^{2}\left[\frac{\partial^{2} \dot{\omega}_{\phi}}{\partial r^{2}}\right]_{r e f}+\Delta \theta \Delta r\left[\frac{\partial^{2} \dot{\omega}_{\phi}}{\partial \theta \partial r}\right]_{r e f}+ \\
& +\Delta \phi \Delta r\left[\frac{\partial^{2} \dot{\omega}_{\phi}}{\partial \phi \partial r}\right]_{r e f}+\Delta v_{r} \Delta r\left[\frac{\partial^{2} \dot{\omega}_{\phi}}{\partial v_{r} \partial r}\right]_{r e f}+\Delta \omega_{\theta} \Delta r\left[\frac{\partial^{2} \dot{\omega}_{\phi}}{\partial \omega_{\theta} \partial r}\right]_{r e f}+ \\
& +\Delta \omega_{\phi} \Delta r\left[\frac{\partial^{2} \dot{\omega}_{\phi}}{\partial \omega_{\phi} \partial r}\right]_{r e f}+\frac{1}{2} \Delta \theta^{2}\left[\frac{\partial^{2} \dot{\omega}_{\phi}}{\partial \theta^{2}}\right]_{r e f}+\Delta \phi \Delta \theta\left[\frac{\partial^{2} \dot{\omega}_{\phi}}{\partial \phi \partial \theta}\right]_{r e f}+ \\
& +\Delta v_{r} \Delta \theta\left[\frac{\partial^{2} \dot{\omega}_{\phi}}{\partial v_{r} \partial \theta}\right]_{r e f}+\Delta \omega_{\theta} \Delta \theta\left[\frac{\partial^{2} \dot{\omega}_{\phi}}{\partial \omega_{\theta} \partial \theta}\right]_{r e f}+\Delta \omega_{\phi} \Delta \theta\left[\frac{\partial^{2} \dot{\omega}_{\phi}}{\partial \omega_{\phi} \partial \theta}\right]_{r e f}+ \\
& +\frac{1}{2} \Delta \phi^{2}\left[\frac{\partial^{2} \dot{\omega}_{\phi}}{\partial \phi^{2}}\right]_{r e f}+\Delta v_{r} \Delta \phi\left[\frac{\partial^{2} \dot{\omega}_{\phi}}{\partial v_{r} \partial \phi}\right]_{r e f}+\Delta \omega_{\theta} \Delta \phi\left[\frac{\partial^{2} \dot{\omega}_{\phi}}{\partial \omega_{\theta} \partial \phi}\right]_{r e f}+ \\
& +\Delta \omega_{\phi} \Delta \phi\left[\frac{\partial^{2} \dot{\omega}_{\phi}}{\partial \omega_{\phi} \partial \phi}\right]_{r e f}+\frac{1}{2} \Delta v_{r}^{2}\left[\frac{\partial^{2} \dot{\omega}_{\phi}}{\partial v_{r}^{2}}\right]_{r e f}+\Delta \omega_{\theta} \Delta v_{r}\left[\frac{\partial^{2} \dot{\omega}_{\phi}}{\partial \omega_{\theta} \partial v_{r}}\right]_{r e f}+ \\
& +\Delta \omega_{\phi} \Delta v_{r}\left[\frac{\partial^{2} \dot{\omega}_{\phi}}{\partial \omega_{\phi} \partial v_{r}}\right]_{r e f}+\frac{1}{2} \Delta \omega_{\theta}^{2}\left[\frac{\partial^{2} \dot{\omega}_{\phi}}{\partial \omega_{\theta}^{2}}\right]_{r e f}+\Delta \omega_{\theta} \Delta \omega_{\phi}\left[\frac{\partial^{2} \dot{\omega}_{\phi}}{\partial \omega_{\phi} \partial \omega_{\theta}}\right]_{r e f}+ \\
& +\frac{1}{2} \Delta \omega_{\phi}^{2}\left[\frac{\partial^{2} \dot{\omega}_{\phi}}{\partial \omega_{\phi}^{2}}\right]_{r e f}
\end{aligned}
$$

where

$$
\left[\frac{\partial^{2} \dot{\omega}_{\phi}}{\partial r^{2}}\right]_{r e f}=\left[-\frac{4 v_{r} \omega_{\phi}}{r^{3}}\right]_{r e f}
$$




$$
=0
$$

$$
\left[\frac{\partial^{2} \dot{\omega}_{\phi}}{\partial \phi \partial r}\right]_{r e f}=0
$$

$$
\begin{aligned}
{\left[\frac{\partial^{2} \dot{\omega}_{\phi}}{\partial v_{r} \partial r}\right]_{r e f} } & =\left[\frac{2 \omega_{\phi}}{r^{2}}\right]_{r e f} \\
& =0
\end{aligned}
$$

$$
\left[\frac{\partial^{2} \dot{\omega}_{\phi}}{\partial \omega_{\theta} \partial r}\right]_{r e f}=0
$$

$$
\left[\frac{\partial^{2} \dot{\omega}_{\phi}}{\partial \omega_{\phi} \partial r}\right]_{r e f}=\left[\frac{2 v_{r}}{r^{2}}\right]_{r e f}
$$$$
=0
$$

$$
\begin{aligned}
{\left[\frac{\partial^{2} \dot{\omega}_{\phi}}{\partial \phi^{2}}\right]_{r e f} } & =\left[4 \omega_{\theta}^{2} \cos \phi \sin \phi\right]_{r e f} \\
& =0
\end{aligned}
$$

$$
\begin{aligned}
{\left[\frac{\partial^{2} \dot{\omega}_{\phi}}{\partial v_{r} \partial \phi}\right]_{r e f} } & =0 \\
{\left[\frac{\partial^{2} \dot{\omega}_{\phi}}{\partial \omega_{\theta} \partial \phi}\right]_{r e f} } & =\left[-2 \omega_{\theta} \cos ^{2} \phi+2 \omega_{\theta} \sin ^{2} \phi\right]_{r e f} \\
& =-2 \omega_{0}
\end{aligned}
$$

$$
\left[\frac{\partial^{2} \dot{\omega}_{\phi}}{\partial \omega_{\phi} \partial \phi}\right]_{r e f}=0
$$

$$
\left[\frac{\partial^{2} \dot{\omega}_{\phi}}{\partial v_{r}^{2}}\right]_{r e f}=0
$$$$
\left[\frac{\partial^{2} \dot{\omega}_{\phi}}{\partial \omega_{\theta} \partial v_{r}}\right]_{r e f}=0
$$

$$
\begin{aligned}
{\left[\frac{\partial^{2} \dot{\omega}_{\phi}}{\partial \omega_{\phi} \partial v_{r}}\right]_{r e f} } & =\left[-\frac{2}{r}\right]_{r e f} \\
& =-\frac{2}{r_{0}}
\end{aligned}
$$




$$
\begin{aligned}
{\left[\frac{\partial^{2} \dot{\omega}_{\phi}}{\partial \omega_{\theta}^{2}}\right]_{r e f} } & =[-2 \sin \phi \cos \phi]_{r e f} \\
& =0 \\
{\left[\frac{\partial^{2} \dot{\omega}_{\phi}}{\partial \omega_{\phi} \partial \omega_{\theta}}\right]_{r e f} } & =0 \\
{\left[\frac{\partial^{2} \dot{\omega}_{\phi}}{\partial \omega_{\phi}^{2}}\right]_{r e f} } & =0
\end{aligned}
$$

which results in

$$
\Delta \dot{\omega}_{\phi}=-\omega_{0}^{2} \Delta \phi-2 \Delta \omega_{\theta} \Delta \phi-\frac{2}{r_{0}} \Delta \omega_{\phi} \Delta v_{r}
$$




\section{Appendix B}

\section{Method of Multiple Scales}

\section{B.1 Evaluation of the First Order PDE}

The first order (in $\epsilon$ ) radial equation was

$$
\begin{aligned}
\frac{\partial^{2} \Delta \hat{r}_{1}}{\partial T_{0}^{2}}-3 \Delta \hat{r}_{1}-2 \frac{\partial \Delta \hat{\theta}_{1}}{\partial T_{0}}= & -2 \frac{\partial^{2} \Delta \hat{r}_{0}}{\partial T_{0} \partial T_{1}}+2 \frac{\partial \Delta \hat{\theta}_{0}}{\partial T_{1}}-3 \Delta \hat{r}_{0}^{2}+2 \Delta \hat{r}_{0} \frac{\partial \Delta \hat{\theta}_{0}}{\partial T_{0}}-\Delta \hat{\phi}_{0}^{2}+ \\
& +\left(\frac{\partial \Delta \hat{\theta}_{0}}{\partial T_{0}}\right)^{2}+\left(\frac{\partial \Delta \hat{\phi}_{0}}{\partial T_{0}}\right)^{2}
\end{aligned}
$$

Expanding each term,

$$
\begin{aligned}
\frac{\partial^{2} \Delta \hat{r}_{0}}{\partial T_{0} \partial T_{1}} & =i \frac{\partial A}{\partial T_{1}} \mathrm{e}^{i T_{0}}-i \frac{\partial \bar{A}}{\partial T_{1}} \mathrm{e}^{-i T_{0}} \\
\frac{\partial \Delta \hat{\theta}_{0}}{\partial T_{1}} & =2 i \frac{\partial A}{\partial T_{1}} \mathrm{e}^{i T_{0}}-2 \frac{\partial \bar{A}}{\partial T_{1}} \mathrm{e}^{-i T_{0}}-\frac{3}{2} \frac{\partial B}{\partial T_{1}} T_{0}+\frac{\partial C}{\partial T_{1}} \\
\Delta \hat{r}_{0}^{2} & =\left(A \mathrm{e}^{i T_{0}}+\bar{A} \mathrm{e}^{-i T_{0}}+B\right)^{2} \\
& =2 A B \mathrm{e}^{i T_{0}}+2 \bar{A} B \mathrm{e}^{-i T_{0}}+A^{2} \mathrm{e}^{2 i T_{0}}+\bar{A}^{2} \mathrm{e}^{-2 i T_{0}}+2 A \bar{A}+B^{2} \\
\Delta \hat{r}_{0} \frac{\partial \Delta \hat{\theta}_{0}}{\partial T_{0}} & =\left(A \mathrm{e}^{i T_{0}}+\bar{A} \mathrm{e}^{-i T_{0}}+B\right)\left(-2 A \mathrm{e}^{i T_{0}}-2 \bar{A} \mathrm{e}^{-i T_{0}}-\frac{3}{2} B\right) \\
& =-\frac{7}{2} A B \mathrm{e}^{i T_{0}}-\frac{7}{2} \bar{A} B \mathrm{e}^{-i T_{0}}-2 A^{2} \mathrm{e}^{2 i T_{0}}-2 \bar{A}^{2} \mathrm{e}^{-2 i T_{0}}-\frac{3}{2} B^{2}-4 A \bar{A} \\
\Delta \hat{\phi}_{0}^{2} & =\left(D \mathrm{e}^{i T_{0}}+\bar{D}^{-i T_{0}}\right)^{2} \\
& =D^{2} \mathrm{e}^{2 i T_{0}}+\bar{D}^{2} \mathrm{e}^{-2 i T_{0}}+2 D \bar{D}
\end{aligned}
$$




$$
\begin{aligned}
\left(\frac{\partial \Delta \hat{\theta}_{0}}{\partial T_{0}}\right)^{2} & =\left(-2 A \mathrm{e}^{i T_{0}}-2 \bar{A} \mathrm{e}^{-i T_{0}}-\frac{3}{2} B\right)^{2} \\
& =6 A B \mathrm{e}^{i T_{0}}+6 \bar{A} B \mathrm{e}^{-i T_{0}}+4 A^{2} \mathrm{e}^{2 i T_{0}}+4 \bar{A}^{2} \mathrm{e}^{-2 i T_{0}}+\frac{9}{4} B^{2}+8 A \bar{A} \\
\left(\frac{\partial \Delta \hat{\phi}_{0}}{\partial T_{0}}\right)^{2} & =\left(i D \mathrm{e}^{i T_{0}}-i \bar{D} \mathrm{e}^{-i T_{0}}\right)^{2} \\
& =-D^{2} \mathrm{e}^{2 i T_{0}}-\bar{D}^{2} \mathrm{e}^{-2 i T_{0}}+2 D \bar{D}
\end{aligned}
$$

Collecting each of these together leads to

$$
\begin{aligned}
\frac{\partial^{2} \Delta \hat{r}_{1}}{\partial T_{0}^{2}}-3 \Delta \hat{r}_{1}-2 \frac{\partial \Delta \hat{\theta}_{1}}{\partial T_{0}}= & \left(2 i \frac{\partial A}{\partial T_{1}}-7 A B\right) \mathrm{e}^{i T_{0}}+\left(-2 i \frac{\partial \bar{A}}{\partial T_{1}}-7 \bar{A} B\right) \mathrm{e}^{-i T_{0}}+ \\
& +\left(-3 A^{2}-2 D^{2}\right) \mathrm{e}^{2 i T_{0}}+\left(-3 \bar{A}^{2}-2 \bar{D}^{2}\right) \mathrm{e}^{-2 i T_{0}}+ \\
& +2 \frac{\partial C}{\partial T_{1}}-\frac{15 B^{2}}{4}-6 A \bar{A}-3 \frac{\partial B}{\partial T_{1}} T_{0}
\end{aligned}
$$

The first order transverse equation was

$$
\begin{aligned}
\frac{\partial^{2} \Delta \hat{\theta}_{1}}{\partial T_{0}^{2}}+2 \frac{\partial \Delta \hat{r}_{1}}{\partial T_{0}}= & -2 \frac{\partial^{2} \Delta \hat{\theta}_{0}}{\partial T_{0} \partial T_{1}}-2 \frac{\partial \Delta \hat{r}_{0}}{\partial T_{1}}+2 \Delta \hat{r}_{0} \frac{\partial \Delta \hat{r}_{0}}{\partial T_{0}}+2 \Delta \hat{\phi}_{0} \frac{\partial \Delta \hat{\phi}_{0}}{\partial T_{0}}- \\
& -2 \frac{\partial \Delta \hat{\theta}_{0}}{\partial T_{0}} \frac{\partial \Delta \hat{r}_{0}}{\partial T_{0}}
\end{aligned}
$$

Expanding each term results in

$$
\begin{aligned}
\frac{\partial^{2} \Delta \hat{\theta}_{0}}{\partial T_{0} \partial T_{1}} & =-2 \frac{\partial A}{\partial T_{1}} \mathrm{e}^{i T_{0}}-2 \frac{\partial \bar{A}}{\partial T_{1}} \mathrm{e}^{-i T_{0}} \\
\frac{\partial \Delta \hat{r}_{0}}{\partial T_{1}} & =\frac{\partial A}{\partial T_{1}} \mathrm{e}^{i T_{0}}+\frac{\partial \bar{A}}{\partial T_{1}} \mathrm{e}^{-i T_{0}}+\frac{\partial B}{\partial T_{1}} \\
\Delta \hat{r}_{0} \frac{\partial \Delta \hat{r}_{0}}{\partial T_{0}} & =\left(A \mathrm{e}^{i T_{0}}+\bar{A} \mathrm{e}^{-i T_{0}}+B\right)\left(i A \mathrm{e}^{i T_{0}}-i \bar{A} \mathrm{e}^{-i T_{0}}\right) \\
& =i A B \mathrm{e}^{i T_{0}}-i \bar{A} B \mathrm{e}^{-i T_{0}}+i A^{2} \mathrm{e}^{2 i T_{0}}-i \bar{A}^{2} \mathrm{e}^{-2 i T_{0}} \\
\Delta \hat{\phi}_{0} \frac{\partial \Delta \hat{\phi}_{0}}{\partial T_{0}} & =\left(D \mathrm{e}^{i T_{0}}+\bar{D} \mathrm{e}^{-i T_{0}}\right)\left(i D \mathrm{e}^{i T_{0}}-i \bar{D} \mathrm{e}^{-i T_{0}}\right) \\
& =i D^{2} \mathrm{e}^{2 i T_{0}}-i \bar{D}^{2} \mathrm{e}^{-2 i T_{0}} \\
\frac{\partial \Delta \hat{\theta}_{0}}{\partial T_{0}} \frac{\partial \hat{r}_{0}}{\partial T_{0}} & =\left(-2 A \mathrm{e}^{i T_{0}}-2 \bar{A} \mathrm{e}^{-i T_{0}}-\frac{3}{2} B\right)\left(i A \mathrm{e}^{i T_{0}}-i \bar{A} \mathrm{e}^{-i T_{0}}\right) \\
& =-\frac{3}{2} i A B \mathrm{e}^{i T_{0}}+\frac{3}{2} i \bar{A} B \mathrm{e}^{-i T_{0}}-2 i A^{2} \mathrm{e}^{2 i T_{0}}+2 i \bar{A}^{2} \mathrm{e}^{-2 i T_{0}}
\end{aligned}
$$


Gathering terms together,

$$
\begin{aligned}
\frac{\partial^{2} \Delta \hat{\theta}_{1}}{\partial T_{0}^{2}}+2 \frac{\partial \Delta \hat{r}_{1}}{\partial T_{0}}= & \left(2 \frac{\partial A}{\partial T_{1}}+5 i A B\right) \mathrm{e}^{i T_{0}}+\left(2 \frac{\partial \bar{A}}{\partial T_{1}}-5 i \bar{A} B\right) \mathrm{e}^{-i T_{0}}+ \\
& +\left(6 i A^{2}+2 i D^{2}\right) \mathrm{e}^{2 i T_{0}}+\left(-6 i \bar{A}^{2}-2 i \bar{D}^{2}\right) \mathrm{e}^{-2 i T_{0}}- \\
& -2 \frac{\partial B}{\partial T_{1}}
\end{aligned}
$$

The first order out of plane equation was

$$
\frac{\partial^{2} \Delta \hat{\phi}_{1}}{\partial T_{0}^{2}}+\Delta \hat{\phi}_{1}=-2 \frac{\partial^{2} \Delta \hat{\phi}_{0}}{\partial T_{0} \partial T_{1}}-2 \Delta \hat{\phi}_{0} \frac{\partial \Delta \hat{\theta}_{0}}{\partial T_{0}}-2 \frac{\partial \Delta \hat{\phi}_{0}}{\partial T_{0}} \frac{\partial \hat{r}_{0}}{\partial T_{0}}
$$

Expanding terms,

$$
\begin{aligned}
\frac{\partial^{2} \Delta \hat{\phi}_{0}}{\partial T_{0} \partial T_{1}} & =i \frac{\partial D}{\partial T_{1}} \mathrm{e}^{i T_{0}}-i \frac{\partial \bar{D}}{\partial T_{1}} \mathrm{e}^{-i T_{0}} \\
\Delta \hat{\phi}_{0} \frac{\partial \Delta \hat{\theta}_{0}}{\partial T_{0}} & =\left(D \mathrm{e}^{i T_{0}}+\bar{D} \mathrm{e}^{-i T_{0}}\right)\left(-2 A \mathrm{e}^{i T_{0}}-2 \bar{A} \mathrm{e}^{-i T_{0}}-\frac{3}{2} B\right) \\
& =-\frac{3}{2} B D \mathrm{e}^{i T_{0}}-\frac{3}{2} B \bar{D} \mathrm{e}^{-i T_{0}}-2 A D \mathrm{e}^{2 i T_{0}}-2 \bar{A} \bar{D} \mathrm{e}^{-2 i T_{0}}-2 A \bar{D}-2 \bar{A} D \\
\frac{\partial \Delta \hat{\phi}_{0}}{\partial T_{0}} \frac{\partial \hat{r}_{0}}{\partial T_{0}} & =\left(i D \mathrm{e}^{i T_{0}}-i \bar{D} \mathrm{e}^{-i T_{0}}\right)\left(i A \mathrm{e}^{i T_{0}}-i \bar{A} \mathrm{e}^{-i T_{0}}\right) \\
& =-A D \mathrm{e}^{i T_{0}}-\bar{A} \bar{D} \mathrm{e}^{-i T_{0}}+A \bar{D}+\bar{A} D
\end{aligned}
$$

All of which results in

$$
\begin{aligned}
\frac{\partial^{2} \Delta \hat{\phi}_{1}}{\partial T_{0}^{2}}+\Delta \hat{\phi}_{1}= & \left(-2 i \frac{\partial D}{\partial T_{1}}+3 D B\right) \mathrm{e}^{i T_{0}}+\left(2 i \frac{\partial \bar{D}}{\partial T_{1}}+3 \bar{D} B\right) \mathrm{e}^{-i T_{0}}+ \\
& +6 A D \mathrm{e}^{2 i T_{0}}+6 \bar{A} \bar{D} \mathrm{e}^{-2 i T_{0}}+2 \bar{A} D+2 A \bar{D}
\end{aligned}
$$

\section{B.2 Evaluation of the Second Order PDE}

The second order radial equation was

$$
\begin{aligned}
\frac{\partial^{2} \Delta \hat{r}_{2}}{\partial T_{0}^{2}}-3 \Delta \hat{r}_{2}-2 \frac{\partial \Delta \hat{\theta}_{2}}{\partial T_{0}}= & -2 \frac{\partial^{2} \Delta \hat{r}_{1}}{\partial T_{0} \partial T_{1}}-2 \frac{\partial^{2} \Delta \hat{r}_{0}}{\partial T_{0} \partial T_{2}}-\frac{\partial^{2} \Delta \hat{r}_{0}}{\partial T_{1}^{2}}+2 \frac{\partial \Delta \hat{\theta}_{0}}{\partial T_{2}}+2 \frac{\partial \Delta \hat{\theta}_{1}}{\partial T_{1}}- \\
& -6 \Delta \hat{r}_{0} \Delta \hat{r}_{1}+2 \Delta \hat{r}_{1} \frac{\partial \Delta \hat{\theta}_{0}}{\partial T_{0}}+2 \Delta \hat{r}_{0} \frac{\partial \Delta \hat{\theta}_{0}}{\partial T_{1}}+2 \Delta \hat{r}_{0} \frac{\partial \Delta \hat{\theta}_{1}}{\partial T_{0}}-
\end{aligned}
$$




$$
\begin{aligned}
& -2 \Delta \hat{\phi}_{0} \Delta \hat{\phi}_{1}+2 \frac{\partial \Delta \hat{\theta}_{0}}{\partial T_{0}} \frac{\partial \Delta \hat{\theta}_{0}}{\partial T_{1}}+2 \frac{\partial \Delta \hat{\theta}_{0}}{\partial T_{0}} \frac{\partial \Delta \hat{\theta}_{1}}{\partial T_{0}}+ \\
& +2 \frac{\partial \Delta \hat{\phi}_{0}}{\partial T_{0}} \frac{\partial \Delta \hat{\phi}_{0}}{\partial T_{1}}+2 \frac{\partial \Delta \hat{\phi}_{0}}{\partial T_{0}} \frac{\partial \Delta \hat{\phi}_{1}}{\partial T_{0}}
\end{aligned}
$$

Expanding each term,

$$
\begin{aligned}
& \frac{\partial^{2} \Delta \hat{r}_{1}}{\partial T_{0} \partial T_{1}}=\left(\frac{27}{4} A^{2} B+\frac{3}{4} A B^{2}+\frac{3}{4} B D^{2}-\frac{3}{4} \bar{A} B^{2}-\frac{3}{4} \bar{A}^{2} B-\frac{3}{4} B \bar{D}^{2}\right) \mathrm{e}^{i T_{0}}+ \\
& +\left(\frac{27}{4} \bar{A}^{2} B+\frac{3}{4} \bar{A} B^{2}+\frac{3}{4} B \bar{D}^{2}-\frac{3}{4} A^{2} B-\frac{3}{4} A B^{2}-\frac{3}{4} B D^{2}\right) \mathrm{e}^{-i T_{0}}- \\
& -6 A^{2} B \mathrm{e}^{2 i T_{0}}-6 \bar{A}^{2} B \mathrm{e}^{-2 i T_{0}} \\
& \frac{\partial^{2} \Delta \hat{r}_{0}}{\partial T_{0} \partial T_{2}}=i \frac{\partial A}{\partial T_{2}} \mathrm{e}^{i T_{0}}-i \frac{\partial \bar{A}}{\partial T_{2}} \\
& \frac{\partial^{2} \Delta \hat{r}_{0}}{\partial T_{1}^{2}}=\frac{9}{4} A B^{2} \mathrm{e}^{i T_{0}}+\frac{9}{4} \bar{A} B^{2} \mathrm{e}^{-i T_{0}} \\
& \frac{\partial \Delta \hat{\theta}_{0}}{\partial T_{2}}=2 i \frac{\partial A}{\partial T_{2}} \mathrm{e}^{i T_{0}}-2 i \frac{\partial \bar{A}}{\partial T_{2}} \mathrm{e}^{-i T_{0}}-\frac{3}{2} \frac{\partial B}{\partial T_{2}} T_{0}+\frac{\partial C}{\partial T_{2}} \\
& \frac{\partial \Delta \hat{\theta}_{1}}{\partial T_{1}}=\left(\frac{27}{2} A^{2} B-\frac{3}{2} A B^{2}+\frac{3}{2} B D^{2}-\frac{3}{2} B^{2} \bar{A}-\frac{3}{2} \bar{A}^{2} B-\frac{3}{2} B \bar{D}^{2}\right) \mathrm{e}^{i T_{0}}+ \\
& +\left(\frac{27}{2} \bar{A}^{2} B+\frac{3}{2} B \bar{D}^{2}-\frac{3}{2} A^{2} B-\frac{3}{2} A B^{2}-\frac{3}{2} B D^{2}-\frac{3}{2} \bar{A} B^{2}\right) \mathrm{e}^{-i T_{0}}+ \\
& +\left(-\frac{15}{2} A^{2} B-\frac{3}{2} B D^{2}\right) \mathrm{e}^{2 i T_{0}}+\left(-\frac{15}{2} \bar{A}^{2} B-\frac{3}{2} B \bar{D}^{2}\right) \mathrm{e}^{-2 i T_{0}}+ \\
& +3 A B^{2}+3 \bar{A} B^{2}-\frac{9}{2} A^{2} B-\frac{9}{2} \bar{A}^{2} B+\frac{3}{2} B D^{2}+\frac{3}{2} B \bar{D}^{2} \\
& \Delta \hat{r}_{0} \Delta \hat{r}_{1}=\left(\frac{5}{4} A^{2} B+\frac{1}{2} A B^{2}-\frac{3}{2} A^{3}-\frac{1}{2} A D^{2}+\frac{1}{4} B D^{2}-A^{2} \bar{A}-A \bar{A} B+\frac{1}{2} B^{2} \bar{A}-\right. \\
& \left.-\frac{3}{2} A \bar{A}^{2}+\frac{1}{4} \bar{A}^{2} B-\frac{1}{2} A \bar{D}^{2}+\frac{1}{4} B \bar{D}^{2}-\frac{i B \Delta \hat{v}_{r_{10}}}{2}-\frac{B \Delta \hat{\omega}_{\theta_{10}}}{4}\right) \mathrm{e}^{i T_{0}}+ \\
& +\left(\frac{1}{4} A^{2} B+\frac{1}{2} A B^{2}+\frac{1}{4} B D^{2}-\frac{3}{2} A^{2} \bar{A}-A \bar{A} B+\frac{1}{2} B^{2} \bar{A}-\frac{1}{2} D^{2} \bar{A}-\right. \\
& \left.-A \bar{A}^{2}+\frac{5}{4} B \bar{A}^{2}-\frac{3}{2} \bar{A}^{3}+\frac{1}{4} B \bar{D}^{2}-\frac{1}{2} \bar{A} \bar{D}^{2}+\frac{i B \Delta \hat{v}_{r_{10}}}{2}-\frac{B \Delta \hat{\omega}_{\theta_{10}}}{4}\right) \mathrm{e}^{-i T_{0}}+ \\
& +\left(\frac{9}{4} A^{3}-\frac{1}{2} A^{2} B+\frac{1}{4} A D^{2}+\frac{1}{2} A \bar{A} B+\frac{1}{4} A \bar{A}^{2}+\frac{1}{4} A \bar{D}^{2}-\right. \\
& \left.-\frac{i A \Delta \hat{v}_{r_{10}}}{2}-\frac{A \Delta \hat{\omega}_{\theta_{10}}}{4}\right) \mathrm{e}^{2 i T_{0}}+
\end{aligned}
$$


Chris Karlgaard

$$
\begin{aligned}
& +\left(\frac{1}{4} A^{2} \bar{A}+\frac{1}{2} A \bar{A} B+\frac{1}{4} \bar{A} D^{2}-\frac{1}{2} \bar{A}^{2} B+\frac{9}{4} \bar{A}^{3}+\frac{1}{4} \bar{A} \bar{D}^{2}+\right. \\
& \left.+\frac{i \bar{A} \Delta \hat{v}_{r_{10}}}{2}-\frac{\bar{A} \Delta \hat{\omega}_{\theta_{10}}}{4}\right) \mathrm{e}^{-2 i T_{0}}- \\
& -A^{3} \mathrm{e}^{3 i T_{0}}-\bar{A}^{3} \mathrm{e}^{-3 i T_{0}}+\frac{1}{4} A^{3}+\frac{1}{4} \bar{A}^{3}-A^{2} B-\bar{A}^{2} B-A B^{2}-\bar{A} B^{2}+ \\
& +\frac{1}{4} A D^{2}+\frac{1}{4} \bar{A} \bar{D}^{2}-\frac{1}{2} B D^{2}-\frac{1}{2} B \bar{D}^{2}+\frac{1}{4} A \bar{D}^{2}+\frac{1}{4} \bar{A} D^{2}+\frac{9}{4} A^{2} \bar{A}+\frac{9}{4} \bar{A}^{2} A+ \\
& +A \bar{A} B+\frac{1}{2} i A \Delta \hat{v}_{r_{10}}-\frac{1}{2} i \bar{A} \Delta \hat{v}_{r_{10}}-\frac{1}{4} A \Delta \hat{\omega}_{\theta_{10}}-\frac{1}{4} \bar{A} \Delta \hat{\omega}_{\theta_{10}} \\
& \Delta \hat{r}_{1} \frac{\partial \Delta \hat{\theta}_{0}}{\partial T_{0}}=\left(3 A^{3}-\frac{11}{8} A^{2} B-\frac{3}{4} A B^{2}+A D^{2}-\frac{3}{8} B D^{2}+2 A^{2} \bar{A}+2 A \bar{A} B-\right. \\
& \left.-\frac{3}{4} \bar{A} B^{2}+3 A \bar{A}^{2}-\frac{3}{8} \bar{A}^{2} B+A \bar{D}^{2}-\frac{3}{8} B \bar{D}^{2}+\frac{3}{4} i B \Delta \hat{v}_{r_{10}}+\frac{3}{8} B \Delta \hat{\omega}_{\theta_{10}}\right) \mathrm{e}^{i T_{0}}+ \\
& +\left(3 A^{2} \bar{A}+2 A \bar{A} B-\frac{3}{8} A^{2} B-\frac{3}{4} A B^{2}-\frac{3}{8} B D^{2}-\frac{3}{4} B^{2} \bar{A}+\bar{A} D^{2}+2 A \bar{A}-\right. \\
& \left.-\frac{11}{8} B \bar{A}^{2}+3 \bar{A}^{3}-\frac{3}{8} B \bar{D}^{2}+\bar{A} \bar{D}^{2}-\frac{3}{4} i B \Delta \hat{v}_{r_{10}}+\frac{3}{8} B \Delta \hat{\omega}_{\theta_{10}}\right) \mathrm{e}^{-i T_{0}}+ \\
& +\left(\frac{1}{2} A^{2} B-\frac{9}{2} A^{3}-\frac{1}{2} A D^{2}-A \bar{A} B-\frac{1}{2} A \bar{A}^{2}-\frac{1}{2} A \bar{D}^{2}+i A \Delta \hat{v}_{r_{10}}+\right. \\
& \left.+\frac{1}{2} A \Delta \hat{\omega}_{\theta_{10}}\right) \mathrm{e}^{2 i T_{0}}+ \\
& +\left(\frac{1}{2} \bar{A}^{2} B-\frac{9}{2} \bar{A}^{3}-\frac{1}{2} \bar{A} \bar{D}^{2}-\frac{1}{2} A^{2} \bar{A}-A \bar{A} B-\frac{1}{2} \bar{A} D^{2}-i \bar{A} \Delta \hat{v}_{r_{10}}+\right. \\
& \left.+\frac{1}{2} \bar{A} \Delta \hat{\omega}_{\theta 10}\right) \mathrm{e}^{-2 i T_{0}}+ \\
& +2 A^{3} \mathrm{e}^{3 i T_{0}}+2 \bar{A}^{3} \mathrm{e}^{-3 i T_{0}}-\frac{1}{2} A^{3}+\frac{5}{4} A^{2} B+\frac{3}{2} A B^{2}-\frac{1}{2} A D^{2}+\frac{3}{4} B D^{2}- \\
& -\frac{9}{2} A^{2} \bar{A}-2 A \bar{A} B+\frac{3}{2} B^{2} \bar{A}-\frac{1}{2} \bar{A} D^{2}-\frac{9}{2} A \bar{A}^{2}+\frac{5}{4} B \bar{A}^{2}-\frac{1}{2} \bar{A}^{3}-\frac{1}{2} A \bar{D}^{2}+ \\
& +\frac{3}{4} B \bar{D}^{2}-\frac{1}{2} \bar{A} \bar{D}^{2}-i A \Delta \hat{v}_{r_{10}}+\frac{1}{2} A \Delta \hat{\omega}_{\theta_{10}}+i A \Delta \hat{v}_{r_{10}}+\frac{1}{2} \bar{A} \Delta \hat{\omega}_{\theta_{10}} \\
& \Delta \hat{r}_{0} \frac{\partial \Delta \hat{\theta}_{0}}{\partial T_{1}}=\left(\frac{9}{4} A^{3}+\frac{3}{2} A^{2} B+\frac{39}{8} A B^{2}+\frac{3}{4} A D^{2}+3 A^{2} \bar{A}+\frac{3}{2} A \bar{A} B+\frac{9}{4} A \bar{A}^{2}+\right. \\
& \left.+\frac{3}{4} A \bar{D}^{2}-\frac{3}{4} A \Delta \hat{\omega}_{\theta_{10}}\right) \mathrm{e}^{i T_{0}}+ \\
& +\left(\frac{9}{4} A^{2} \bar{A}+\frac{3}{2} A \bar{A} B+\frac{39}{8} B^{2} \bar{A}+\frac{3}{4} \bar{A} D^{2}+3 A \bar{A}^{2}+\frac{3}{2} B \bar{A}^{2}+\frac{9}{4} \bar{A}^{3}+\right. \\
& \left.+\frac{3}{4} \bar{A} \bar{D}^{2}-\frac{3}{4} \bar{A} \Delta \hat{\omega}_{\theta_{10}}\right) \mathrm{e}^{-i T_{0}}+3 A^{2} B \mathrm{e}^{2 i T_{0}}+3 \bar{A}^{2} B \mathrm{e}^{-2 i T_{0}}+\frac{9}{4} A^{2} B+ \\
& +\frac{3}{2} A B^{2}+\frac{15}{8} B^{3}+\frac{3}{4} B D^{2}+9 A \bar{A} B+\frac{3}{2} B^{2} \bar{A}+\frac{9}{4} B \bar{A}^{2}+\frac{3}{4} B \bar{D}^{2}
\end{aligned}
$$




$$
\begin{aligned}
\Delta \hat{\phi}_{0} \Delta \hat{\phi}_{1}= & 2 \bar{A} D^{2} \mathrm{e}^{i T_{0}}+2 A \bar{D}^{2} \mathrm{e}^{-i T_{0}}+ \\
& +\left(3 A D^{2}-\bar{A} D^{2}-A D \bar{D}-D \bar{A} \bar{D}-\frac{1}{2} i D \Delta \hat{\omega}_{\phi_{10}}\right) \mathrm{e}^{2 i T_{0}}+ \\
& +\left(3 \bar{A} \bar{D}^{2}-A D \bar{D}-D \bar{A} \bar{D}-A \bar{D}^{2}+\frac{1}{2} i \bar{D} \Delta \hat{\omega}_{\theta_{10}}\right) \mathrm{e}^{-2 i T_{0}}- \\
& -2 A D^{2} \mathrm{e}^{3 i T_{0}}-2 \bar{A} \bar{D}^{2} \mathrm{e}^{-3 i T_{0}}-A D^{2}-\bar{A} D^{2}+2 D \bar{A} \bar{D}-A \bar{D}^{2}- \\
& -\bar{A} \bar{D}^{2}+\frac{1}{2} i D \Delta \hat{\phi}_{\theta_{10}}-\frac{1}{2} i \bar{D} \Delta \hat{\omega}_{\phi_{10}}
\end{aligned}
$$

$$
\begin{aligned}
\frac{\partial \Delta \hat{\theta}_{0}}{\partial T_{0}} \frac{\partial \Delta \hat{\theta}_{0}}{\partial T_{1}}= & \left(\frac{3}{2} A \Delta \hat{\omega}_{\theta_{10}}-\frac{9}{2} A^{3}-3 A^{2} B-\frac{33}{4} A B^{2}-\frac{3}{2} A D^{2}-6 A^{2} \bar{A}-3 A \bar{A} B-\right. \\
& \left.-\frac{9}{2} A \bar{A}^{2}-\frac{3}{2} A \bar{D}^{2}\right) \mathrm{e}^{i T_{0}}+\left(\frac{3}{2} \bar{A} \Delta \hat{\omega}_{\theta_{10}}-\frac{9}{2} A^{2} \bar{A}-3 A \bar{A} B-\frac{33}{4} B^{2} \bar{A}-\right. \\
& \left.-\frac{3}{2} D^{2} \bar{A}-6 A \bar{A}^{2}-3 B \bar{A}^{2}-\frac{9}{4} \bar{A}^{3}-\frac{3}{2} \bar{A} \bar{D}^{2}\right) \mathrm{e}^{-i T_{0}}-6 A^{2} B \mathrm{e}^{2 i T_{0}}- \\
& -6 \bar{A}^{2} B \mathrm{e}^{-2 i T_{0}}-\frac{27}{8} A^{2} B-\frac{9}{4} A B^{2}-\frac{45}{16} B^{3}-\frac{9}{8} B D^{2}-\frac{33}{2} A \bar{A} B- \\
& -\frac{9}{4} B^{2} \bar{A}-\frac{27}{8} B \bar{A}^{2}-\frac{9}{8} B \bar{D}^{2}+\frac{9}{8} B \Delta \hat{\omega}_{\theta_{10}}
\end{aligned}
$$

$$
\begin{aligned}
\frac{\partial \Delta \hat{\theta}_{0}}{\partial T_{0}} \frac{\partial \Delta \hat{\theta}_{1}}{\partial T_{0}}= & \left(\frac{27}{4} A^{2} B-\frac{3}{2} A B^{2}+\frac{3}{4} B D^{2}-10 A^{2} \bar{A}+\frac{3}{2} B^{2} \bar{A}-2 \bar{A} D^{2}+\frac{3}{4} \bar{A}^{2} B+\right. \\
& \left.+\frac{3}{4} B \bar{D}^{2}-\frac{3}{2} i B \Delta \hat{v}_{r_{10}}-\frac{3}{4} B \Delta \hat{\omega}_{\theta_{10}}\right) \mathrm{e}^{i T_{0}}+\left(\frac{3}{4} A^{2} B+\frac{15}{4} A B^{2}+\frac{3}{4} B D^{2}-\right. \\
& -\frac{15}{4} \bar{A} B^{2}-10 A \bar{A}^{2}+\frac{27}{4} B \bar{A}^{2}-2 A \bar{D}^{2}+\frac{3}{4} B \bar{D}^{2}+\frac{3}{2} i B \Delta \hat{v}_{r_{10}}- \\
& \left.-\frac{3}{4} B \Delta \hat{\omega}_{\theta_{10}}\right) \mathrm{e}^{-i T_{0}}+\left(9 A^{3}-\frac{19}{2} A^{2} B+A D^{2}-\frac{3}{2} B D^{2}+2 A \bar{A} B+\right. \\
& \left.+A \bar{A}^{2}+A \bar{D}^{2}-2 i A \Delta \hat{v}_{r_{10}}-A \Delta \hat{\omega}_{\theta_{10}}\right) \mathrm{e}^{2 i T_{0}}+\left(A^{2} \bar{A}+5 A \bar{A} B+\bar{A} D^{2}-\right. \\
& \left.-\frac{25}{2} B \bar{A}^{2}+9 \bar{A}^{3}-\frac{3}{2} B \bar{D}^{2}+\bar{A} \bar{D}^{2}+2 i \bar{A} \Delta \hat{v}_{r_{10}}-\bar{A} \Delta \hat{\omega}_{\theta_{10}}\right) \mathrm{e}^{-2 i T_{0}}+ \\
& +\left(-10 A^{3}-2 A D^{2}\right) \mathrm{e}^{3 i T_{0}}+\left(-10 \bar{A}^{3}-2 \bar{A} \bar{D}^{2}\right) \mathrm{e}^{-3 i T_{0}}+ \\
& +A^{3}+5 A^{2} B+A D^{2}+9 A^{2} b a r A-7 A \bar{A} B+\bar{A} D^{2}+9 A \bar{A}^{2}+2 B \bar{A}^{2}+ \\
& +\bar{A}^{3}+A \bar{D}^{2}+\bar{A} \bar{D}^{2}+2 i A \Delta \hat{v}_{r_{10}}-2 i \bar{A} \Delta \hat{v}_{r_{10}}-A \Delta \hat{\omega}_{\theta_{10}}-\bar{A} \Delta \hat{\omega}_{\theta_{10}}
\end{aligned}
$$

$\frac{\partial \Delta \hat{\phi}_{0}}{\partial T_{0}} \frac{\partial \Delta \hat{\phi}_{0}}{\partial T_{1}}=\frac{3}{2} B D^{2} \mathrm{e}^{2 i T_{0}}+\frac{3}{2} B \bar{D}^{2} \mathrm{e}^{-2 i T_{0}}-3 B D \bar{D}$

$$
\frac{\partial \Delta \hat{\phi}_{0}}{\partial T_{0}} \frac{\partial \Delta \hat{\phi}_{1}}{\partial T_{0}}=-4 A D \bar{D} \mathrm{e}^{i T_{0}}-4 \bar{A} D \bar{D} \mathrm{e}^{-i T_{0}}+
$$


Chris Karlgaard

$$
\begin{aligned}
& +\left(\bar{A} D^{2}+A D \bar{D}+D \bar{A} \bar{D}-3 A D^{2}+\frac{1}{2} i D \Delta \hat{\omega}_{\phi_{10}}\right) \mathrm{e}^{2 i T_{0}}+ \\
& +\left(A D \bar{A}+D \bar{A} \bar{D}+A \bar{D}^{2}-3 \bar{A} \bar{D}^{2}-\frac{1}{2} i \bar{D} \Delta \hat{\omega}_{\theta_{10}}\right) \mathrm{e}^{-2 i T_{0}}+ \\
& +4 A D^{2} \mathrm{e}^{3 i T_{0}}+4 \bar{A} \bar{D}^{2} \mathrm{e}^{-3 i T_{0}}-A D^{2}-D^{2} \bar{A}+2 A D \bar{D}+2 D \bar{A} \bar{D}- \\
& -A \bar{D}^{2}-\bar{A} \bar{D}^{2}+\frac{1}{2} i D \Delta \hat{\omega}_{\phi_{10}}-\frac{1}{2} i \bar{D} \Delta \hat{\omega}_{\phi_{10}}
\end{aligned}
$$

Collecting these results leads to the definitions for $\gamma_{r_{2}}, \eta_{r_{2}}, \chi_{r_{2}}$ and $\sigma_{r_{2}}$ given in chapter (4). The second order transverse equation was

$$
\begin{aligned}
\frac{\partial^{2} \Delta \hat{\theta}_{2}}{\partial T_{0}^{2}}+2 \frac{\partial \Delta \hat{r}_{2}}{\partial T_{0}}= & -2 \frac{\partial^{2} \Delta \hat{\theta}_{1}}{\partial T_{0} \partial T_{1}}-2 \frac{\partial^{2} \Delta \hat{\theta}_{0}}{\partial T_{0} \partial T_{2}}-\frac{\partial^{2} \Delta \hat{\theta}_{0}}{\partial T_{1}^{2}}-2 \frac{\partial \Delta \hat{r}_{0}}{\partial T_{2}}-2 \frac{\partial \Delta \hat{r}_{1}}{\partial T_{1}}+ \\
& +2 \Delta \hat{r}_{0} \frac{\partial \Delta \hat{r}_{1}}{\partial T_{0}}+2 \Delta \hat{r}_{0} \frac{\partial \Delta \hat{r}_{0}}{\partial T_{1}}+2 \Delta \hat{r}_{1} \frac{\partial \Delta \hat{r}_{0}}{\partial T_{0}}+2 \Delta \hat{\phi}_{0} \frac{\partial \Delta \hat{\phi}_{1}}{\partial T_{0}}+ \\
& +2 \Delta \hat{\phi}_{0} \frac{\partial \Delta \hat{\phi}_{0}}{\partial T_{1}}+2 \Delta \hat{\phi}_{1} \frac{\partial \Delta \hat{\phi}_{0}}{\partial T_{0}}-2 \frac{\partial \Delta \hat{\theta}_{0}}{\partial T_{0}} \frac{\partial \Delta \hat{r}_{1}}{\partial T_{0}}- \\
& -2 \frac{\partial \Delta \hat{\theta}_{0}}{\partial T_{0}} \frac{\partial \Delta \hat{r}_{0}}{\partial T_{1}}-2 \frac{\partial \Delta \hat{\theta}_{1}}{\partial T_{0}} \frac{\partial \Delta \hat{r}_{0}}{\partial T_{0}}-2 \frac{\partial \Delta \hat{\theta}_{0}}{\partial T_{1}} \frac{\partial \Delta \hat{r}_{0}}{\partial T_{0}}
\end{aligned}
$$

Expanding terms

$$
\begin{aligned}
\frac{\partial^{2} \Delta \hat{\theta}_{1}}{\partial T_{0} \partial T_{1}}= & \left(\frac{3}{2} A B^{2}-\frac{27}{2} A^{2} B-\frac{3}{2} B D^{2}+\frac{3}{2} B^{2} \bar{A}+\frac{3}{2} B \bar{A}^{2}+\frac{3}{2} B \bar{D}^{2}\right) i \mathrm{e}^{i T_{0}}+ \\
& +\left(\frac{3}{2} A^{2} B+\frac{3}{2} A B^{2}+\frac{3}{2} B D^{2}+\frac{3}{2} B^{2} \bar{A}-\frac{27}{2} B \bar{A}^{2}-\frac{3}{2} B \bar{D}^{2}\right) i \mathrm{e}^{-i T_{0}}+ \\
& +\left(-15 A^{2} B-3 B D^{2}\right) i \mathrm{e}^{2 i T_{0}}+\left(15 \bar{A}^{2} B+3 B \bar{D}^{2}\right) i \mathrm{e}^{-2 i T_{0}} \\
\frac{\partial^{2} \Delta \hat{\theta}_{0}}{\partial T_{0} \partial T_{2}}= & -2 \frac{\partial A}{\partial T_{1}} \mathrm{e}^{i T_{0}}-2 \frac{\partial \bar{A}}{\partial T_{1}} \mathrm{e}^{-i T_{0}}-\frac{3}{2} \frac{\partial B}{\partial T_{2}} \\
\frac{\partial^{2} \Delta \hat{\theta}_{0}}{\partial T_{1}^{2}}= & \frac{9}{2} i A B^{2} \mathrm{e}^{i T_{0}}-\frac{9}{2} i \bar{A} B \mathrm{e}^{-i T_{0}}+\frac{27}{4} i B \bar{A}^{2}-\frac{27}{4} i A^{2} B- \\
& -\frac{45}{4} i A B^{2}+\frac{45}{4} i B^{2} \bar{A}-\frac{9}{4} i B D^{2}+\frac{9}{4} i B \bar{D}^{2} \\
\frac{\partial \Delta \hat{r}_{0}}{\partial T_{2}}= & \frac{\partial A}{\partial T_{2}} \mathrm{e}^{i T_{0}}+\frac{\partial \bar{A}}{\partial T_{2}} \mathrm{e}^{-i T_{0}}+\frac{\partial B}{\partial T_{2}} \\
\frac{\partial \Delta \hat{r}_{1}}{\partial T_{1}}= & \left(\frac{3}{4} B^{2} \bar{A}+\frac{3}{4} B \bar{A}^{2}+\frac{3}{4} B \bar{D}^{2}-\frac{27}{4} A^{2} B-\frac{3}{4} A B^{2}-\frac{3}{4} B D^{2}\right) i \mathrm{e}^{i T_{0}}+ \\
& +\left(\frac{3}{4} B^{2} \bar{A}+\frac{27}{4} B \bar{A}^{2}+\frac{3}{4} B \bar{D}^{2}-\frac{3}{4} A^{2} B-\frac{3}{4} A B^{2}-\frac{3}{4} B D^{2}\right) i \mathrm{e}^{-i T_{0}}+ \\
& +3 i A^{2} B \mathrm{e}^{2 i T_{0}}-3 i \bar{A}^{2} B \mathrm{e}^{-2 i T_{0}}+\frac{9}{2} i A^{2} B-\frac{9}{2} i \bar{A}^{2} B+\frac{3}{2} i A B^{2}+
\end{aligned}
$$




$$
\begin{aligned}
& +\frac{3}{2} i B D^{2}-\frac{3}{2} i B^{2} \bar{A}-\frac{3}{2} i B \bar{D}^{2} \\
& \Delta \hat{r}_{0} \frac{\partial \Delta \hat{r}_{1}}{\partial T_{0}}=\left(\frac{9}{4} A^{2} B+\frac{1}{2} A B^{2}+\frac{1}{4} B D^{2}-2 A^{2} \bar{A}+\frac{1}{2} B^{2} \bar{A}+\frac{1}{4} B \bar{A}^{2}+\frac{1}{4} B \bar{D}^{2}-\right. \\
& \left.-i \frac{1}{2} B \Delta \hat{v}_{r_{10}}-\frac{1}{4} B \Delta \hat{\omega}_{\theta_{10}}\right) i \mathrm{e}^{i T_{0}}+\left(2 A \bar{A}^{2}-\frac{9}{4} A \bar{A}^{2}-\frac{1}{4} B \bar{D}^{2}-\right. \\
& \left.-\frac{1}{4} A^{2} B-\frac{1}{2} A B^{2}-\frac{1}{4} B D^{2}-\frac{1}{2} B^{2} \bar{A}-i \frac{1}{2} B \Delta \hat{v}_{r_{10}}+\frac{1}{4} B \Delta \hat{\omega}_{\theta_{10}}\right) i \mathrm{e}^{-i T_{0}}+ \\
& +\left(\frac{9}{4} A^{3}-\frac{3}{2} A^{2} B+\frac{1}{4} A D^{2}+\frac{1}{2} A B \bar{A}+\frac{1}{4} A \bar{A}^{2}+\frac{1}{4} A \bar{D}^{2}-i \frac{1}{2} A \Delta \hat{v}_{r_{10}}-\right. \\
& \left.-\frac{1}{4} A \Delta \hat{\omega}_{\theta_{10}}\right) i \mathrm{e}^{2 i T_{0}}+\left(\frac{3}{2} B \bar{A}^{2}-\frac{9}{4} \bar{A}^{3}-\frac{1}{4} \bar{A} \bar{D}^{2}-\frac{1}{4} A^{2} \bar{A}-\frac{1}{2} A \bar{A} B-\frac{1}{4} D^{2} \bar{A}-\right. \\
& \left.-\frac{1}{2} i \bar{A} \Delta \hat{v}_{r_{10}}+\frac{1}{4} \bar{A} \Delta \hat{\omega}_{\theta_{10}}\right) i \mathrm{e}^{-2 i T_{0}}-2 i A^{3} \mathrm{e}^{3 i T_{0}}+2 i \bar{A}^{3} \mathrm{e}^{-3 i T_{0}}+ \\
& +\left(\frac{9}{4} A^{2} \bar{A}+\frac{1}{4} D^{2} \bar{A}+\frac{1}{2} B \bar{A}^{2}+\frac{1}{4} \bar{A}^{3}-\frac{1}{4} A^{3}-\frac{1}{2} A^{2} B-\frac{1}{4} A D^{2}-\frac{9}{4} A \bar{A}^{2}-\right. \\
& \left.-\frac{1}{4} A \bar{D}^{2}+\frac{1}{4} \bar{A} \bar{D}^{2}+\frac{1}{4} A \Delta \hat{\omega}_{\theta_{10}}-\frac{1}{4} \Delta \hat{\omega}_{\theta_{10}}\right) i+\frac{1}{2} A \Delta \hat{v}_{r_{10}}+\frac{1}{2} \bar{A} \Delta \hat{v}_{r_{10}} \\
& \Delta \hat{r}_{0} \frac{\partial \Delta \hat{r}_{0}}{\partial T_{1}}=-\frac{3}{2} i A B^{2} \mathrm{e}^{i T_{0}}+\frac{3}{2} i \bar{A} B^{2} \mathrm{e}^{-i T_{0}}-\frac{3}{2} i A^{2} B \mathrm{e}^{2 i T_{0}}+\frac{3}{2} i \bar{A}^{2} B \mathrm{e}^{-2 i T_{0}} \\
& \Delta \hat{r}_{1} \frac{\partial \Delta \hat{r}_{0}}{\partial T_{0}}=\left(A^{2} \bar{A}-\frac{3}{2} A^{3}-A^{2} B-\frac{1}{2} A D^{2}-A \bar{A} B-\frac{3}{2} A \bar{A}^{2}-\frac{1}{2} A \bar{D}^{2}\right) i \mathrm{e}^{i T_{0}}+ \\
& +\left(\frac{3}{2} A^{2} \bar{A}+A \bar{A} B+\frac{1}{2} \bar{A} D^{2}-A \bar{A}^{2}+B \bar{A}^{2}+\frac{3}{2} \bar{A}^{3}+\frac{1}{2} \bar{A} \bar{D}^{2}\right) i \mathrm{e}^{-i T_{0}}+ \\
& +\left(\frac{9}{4} A^{3}+\frac{1}{2} A^{2} B+\frac{1}{4} A D^{2}+\frac{1}{2} A \bar{A} B+\frac{1}{4} A \bar{A}^{2}+\frac{1}{4} A \bar{D}^{2}-i \frac{1}{2} A \Delta \hat{v}_{r_{10}}-\right. \\
& \left.-\frac{1}{4} A \Delta \hat{\omega}_{\theta_{10}}\right) i \mathrm{e}^{2 i T_{0}}+\left(\frac{1}{4} \bar{A} \Delta \hat{\omega}_{\theta_{10}}-\frac{1}{2} i \bar{A} \Delta \hat{v}_{r_{10}}-\frac{1}{4} A^{2} \bar{A}-\frac{1}{2} A \bar{A} B-\right. \\
& \left.-\frac{1}{4} \bar{A} D^{2}-\frac{1}{2} B \bar{A}^{2}-\frac{9}{4} \bar{A}^{3}-\frac{1}{4} \bar{A} \bar{D}^{2}\right) \mathrm{e}^{-2 i T_{0}}-i A^{3} \mathrm{e}^{3 i T_{0}}+i \bar{A}^{3} \mathrm{e}^{-3 i T_{0}}+ \\
& +\left(\frac{1}{4} A^{3}+\frac{1}{2} A^{2} B+\frac{1}{4} A D^{2}-\frac{9}{4} A^{2} \bar{A}-\frac{1}{4} D^{2} \bar{A}+\frac{9}{4} A \bar{A}^{2}-\frac{1}{2} B \bar{A}^{2}-\frac{1}{4} \bar{A}^{3}+\right. \\
& \left.+\frac{1}{4} \bar{D}^{2} A-\frac{1}{4} \bar{A} \bar{D}^{2}-\frac{1}{4} A \Delta \hat{\omega}_{\theta_{10}}+\frac{1}{4} \bar{A} \Delta \hat{\omega}_{\theta_{10}}\right) i-\frac{1}{2} A \Delta \hat{v}_{r_{10}}-\frac{1}{2} \bar{A} \Delta \hat{v}_{r_{10}}
\end{aligned}
$$

$$
\begin{aligned}
\Delta \hat{\phi}_{0} \frac{\partial \Delta \hat{\phi}_{1}}{\partial T_{0}}= & 4 i A D \bar{D} \mathrm{e}^{i T_{0}}-4 i \bar{A} \bar{D} D \mathrm{e}^{-i T_{0}}+ \\
& +\left(3 A D^{2}-D^{2} \bar{A}-A D \bar{D}-\bar{A} D \bar{D}+\frac{1}{2} D \Delta \hat{\omega}_{\phi_{10}}\right) i \mathrm{e}^{2 i T_{0}}+ \\
& +\left(\bar{A} D \bar{D}+A D \bar{D}+A \bar{D}^{2}-3 \bar{A} \bar{D}^{2}+\frac{1}{2} \bar{D} \Delta \hat{\omega}_{\phi_{10}}\right) i \mathrm{e}^{-2 i T_{0}}- \\
& -4 i A D^{2} \mathrm{e}^{3 i T_{0}}+4 i \bar{A} \bar{D}^{2} \mathrm{e}^{-3 i T_{0}}+\left(A D^{2}+\bar{A} D^{2}+4 A D \bar{D}-\right.
\end{aligned}
$$




$$
\left.-4 \bar{A} \bar{D} D-A \bar{D}^{2}-\bar{A} \bar{D}^{2}\right)+\frac{1}{2} D \Delta \hat{\omega}_{\phi_{10}}+\frac{1}{2} \bar{D} \Delta \hat{\omega}_{\phi_{10}}
$$

$$
\begin{aligned}
\Delta \hat{\phi}_{0} \frac{\partial \Delta \hat{\phi}_{0}}{\partial T_{1}}= & -\frac{3}{2} i B D^{2} \mathrm{e}^{2 i T_{0}}+\frac{3}{2} i B \bar{D}^{2} \mathrm{e}^{-2 i T_{0}} \\
\Delta \hat{\phi}_{1} \frac{\partial \Delta \hat{\phi}_{0}}{\partial T_{0}}= & \left(2 D^{2} \bar{A}+4 A D \bar{D}\right) i \mathrm{e}^{i T_{0}}-\left(4 D A \bar{D}+2 A \bar{D}^{2}\right) i \mathrm{e}^{-i T_{0}}+ \\
& +\left(3 A D^{2}-D^{2} \bar{A}-A D \bar{D}-D \bar{A} \bar{D}+\frac{1}{2} D \Delta \hat{\omega}_{\phi_{10}}\right) i \mathrm{e}^{2 i T_{0}}+ \\
& +\left(A D \bar{D}+D \bar{A} \bar{D}+A \bar{D}^{2}-3 \bar{A} \bar{D}^{2}+\frac{1}{2} \bar{D} \Delta \hat{\omega}_{\phi_{10}}\right) \mathrm{e}^{-2 i T_{0}}- \\
& -2 i A D^{2} \mathrm{e}^{3 i T_{0}}+2 i \bar{A} \bar{D}^{2} \mathrm{e}^{-3 i T_{0}}+\left(4 D \bar{D} \bar{A}+A \bar{D}^{2}+\bar{A} \bar{D}^{2}-A D^{2}-\right. \\
& \left.-D^{2} \bar{A}-4 A D \bar{D}\right) i-\frac{1}{2} D \Delta \hat{\omega}_{\phi_{10}}-\frac{1}{2} \bar{D} \Delta \hat{\omega}_{\phi_{10}}
\end{aligned}
$$

$$
\begin{aligned}
\frac{\partial \Delta \hat{\theta}_{0}}{\partial T_{0}} \frac{\partial \Delta \hat{r}_{0}}{\partial T_{1}}= & \left(4 A^{2} \bar{A}-\frac{27}{8} A^{2} B-\frac{3}{4} A B^{2}-\frac{3}{8} B D^{2}-\frac{3}{4} B^{2} \bar{A}-\frac{3}{8} B \bar{A}^{2}-\right. \\
& \left.-\frac{3}{8} B \bar{D}^{2}+\frac{3}{4} i B \Delta \hat{v}_{r_{10}}+\frac{3}{8} B \Delta \hat{\omega}_{\theta_{10}}\right) i \mathrm{e}^{i T_{0}}+\left(\frac{3}{8} A^{2} B+\frac{3}{4} A B^{2}+\frac{3}{8} B D^{2}+\right. \\
& \left.+\frac{3}{4} B^{2} \bar{A}-4 A \bar{A}^{2}+\frac{27}{8} B \bar{A}^{2}+\frac{3}{8} B \bar{D}^{2}+\frac{3}{4} i B \Delta \hat{v}_{r_{10}}-\frac{3}{8} B \Delta \hat{\omega}_{\theta_{10}}\right) i \mathrm{e}^{-i T_{0}}+ \\
& +\left(2 A^{2} B-\frac{9}{2} A^{3}-\frac{1}{2} A D^{2}+i A \Delta \hat{v}_{r_{10}}+\frac{1}{2} A \Delta \hat{\omega}_{\theta_{10}}-A \bar{A} B-\frac{1}{2} A \bar{A}^{2}-\right. \\
& \left.-\frac{1}{2} A \bar{D}^{2}\right) \mathrm{e}^{2 i T_{0}}+\left(\frac{1}{2} A^{2} \bar{A}+A \bar{A} B+\frac{1}{2} D^{2} \bar{A}-\frac{1}{2} \bar{A} \bar{D}^{2}+\frac{9}{2} \bar{A}^{3}+i \bar{A} \Delta \hat{v}_{r_{10}}-\right. \\
& \left.-\frac{1}{2} \bar{A} \Delta \hat{\omega}_{\theta_{10}}-2 B \bar{A}^{2}\right) \mathrm{e}^{-2 i T_{0}}+4 i A^{3} \mathrm{e}^{3 i T_{0}}-4 i \bar{A}^{3} \mathrm{e}^{-3 i T_{0}}+\left(\frac{1}{2} A^{3}+A^{2} B+\right. \\
& +\frac{1}{2} A D^{2}-\frac{9}{2} A^{2} \bar{A}-\frac{1}{2} D^{2} \bar{A}+\frac{9}{2} A \bar{A}^{2}-B \bar{A}^{2}-\frac{1}{2} \bar{A}^{3}+\frac{1}{2} A \bar{D}^{2}- \\
& \left.-\frac{1}{2} \bar{A} \bar{D}^{2}-\frac{1}{2} A \Delta \hat{\omega}_{\theta_{10}}+\frac{1}{2} \bar{A} \Delta \hat{\omega}_{\theta_{10}}\right) i-A \Delta \hat{v}_{r_{10}}-\bar{A} \Delta \hat{v}_{r_{10}}
\end{aligned}
$$

$$
\begin{aligned}
\frac{\partial \Delta \hat{\theta}_{0}}{\partial T_{0}} \frac{\partial \Delta \hat{r}_{0}}{\partial T_{1}}= & \frac{9}{4} i A B^{2} \mathrm{e}^{i T_{0}}-\frac{9}{4} i \bar{A} B^{2} \mathrm{e}^{-i T_{0}}+3 i A^{2} B \mathrm{e}^{2 i T_{0}}-3 i \bar{A}^{2} B \mathrm{e}^{-2 i T_{0}} \\
\frac{\partial \Delta \hat{\theta}_{1}}{\partial T_{0}} \frac{\partial \Delta \hat{r}_{0}}{\partial T_{0}}= & -\left(5 A^{2} \bar{A}+D^{2} \bar{A}\right) i \mathrm{e}^{i T_{0}}+\left(5 A \bar{A}^{2}+A \bar{D}^{2}\right) i \mathrm{e}^{-i T_{0}}+ \\
& +\left(A^{2} B-\frac{9}{2} A^{3}-\frac{1}{2} A D^{2}-A B \bar{A}-\frac{1}{2} A \bar{A}^{2}-\frac{1}{2} A \bar{D}^{2}+i A \Delta \hat{v}_{r_{10}}+\right. \\
& \left.+\frac{1}{2} A \Delta \hat{\omega}_{\theta_{10}}\right) i \mathrm{e}^{2 i T_{0}}+\left(\frac{1}{2} A^{2} \bar{A}+\frac{5}{2} A B \bar{A}+\frac{1}{2} D^{2} \bar{A}-\frac{5}{2} B \bar{A}^{2}+\frac{9}{2} \bar{A}^{3}+\frac{1}{2} \bar{A} \bar{D}^{2}-\right. \\
& \left.-\frac{1}{2} \bar{A} \Delta \hat{\omega}_{\theta_{10}}+i \Delta \hat{v}_{r_{10}}\right) i \mathrm{e}^{-2 i T_{0}}+\left(5 A^{3}+A D^{2}\right) i \mathrm{e}^{3 i T_{0}}-\left(5 \bar{A}^{3}+\bar{A}^{2}\right) i \mathrm{e}^{-3 i T_{0}}+
\end{aligned}
$$




$$
\begin{aligned}
+ & \left(\frac{9}{2} A^{2} \bar{A}-\frac{1}{2} A^{3}-\frac{5}{2} A^{2} B-\frac{1}{2} A D^{2}+\frac{3}{2} A B \bar{A}+\frac{1}{2} D^{2} \bar{A}-\frac{9}{2} A \bar{A}^{2}+B \bar{A}^{2}+\right. \\
& \left.+\frac{1}{2} \bar{A}^{3}-\frac{1}{2} A \bar{D}^{2}+\frac{1}{2} \bar{A} \bar{D}^{2}+\frac{1}{2} A \Delta \hat{\omega}_{\theta_{10}}-\frac{1}{2} \bar{A} \Delta \hat{\omega}_{\theta_{10}}\right) i+A \Delta \hat{v}_{r_{10}}+\bar{A} \Delta \hat{v}_{r_{10}} \text { (B.51) } \\
\frac{\partial \Delta \hat{\theta}_{0}}{\partial T_{1}} \frac{\partial \Delta \hat{r}_{0}}{\partial T_{0}}= & \frac{9}{4} A^{3}+\frac{3}{2} A^{2} B+\frac{15}{8} A B^{2}+\frac{3}{4} A D^{2}+3 A^{2} \bar{A}+\frac{3}{2} A \bar{A} B+\frac{9}{4} A \bar{A}^{2}+ \\
& \left.+\frac{3}{4} A \bar{D}^{2}-\frac{3}{4} A \Delta \hat{\omega}_{\theta_{10}}\right) \mathrm{e}^{i T_{0}}+\left(\frac{3}{4} D^{2} \bar{A}-\frac{9}{4} A^{2} \bar{A}-\frac{3}{2} A B \bar{A}-\frac{15}{8} B^{2} \bar{A}-3 A \bar{A}^{2}-\right. \\
& \left.-\frac{3}{2} B \bar{A}^{2}-\frac{9}{4} \bar{A}^{3}-\frac{3}{4} \bar{A} \bar{D}^{2}+\frac{3}{4} \bar{A} \Delta \hat{\omega}_{\theta_{10}}\right) i \mathrm{e}^{-i T_{0}}+3 i A^{2} B \mathrm{e}^{2 i T_{0}}-3 \bar{A}^{2} B \mathrm{e}^{-2 i T_{0}}(\mathrm{~B} .52)
\end{aligned}
$$

Which results in the definitions for $\gamma_{\theta_{2}}, \eta_{\theta_{2}}, \chi_{\theta_{2}}$ and $\sigma_{\theta_{2}}$ found in chapter (4). The second order out of plane equation was

$$
\begin{aligned}
\frac{\partial^{2} \Delta \hat{\phi}_{2}}{\partial T_{0}^{2}}+\Delta \hat{\phi}_{2}= & -2 \frac{\partial^{2} \Delta \hat{\phi}_{1}}{\partial T_{0} \partial T_{1}}-2 \frac{\partial^{2} \Delta \hat{\phi}_{0}}{\partial T_{0} \partial T_{2}}-\frac{\partial^{2} \Delta \hat{\phi}_{0}}{\partial T_{1}^{2}}-2 \Delta \hat{\phi}_{1} \frac{\partial \Delta \hat{\theta}_{0}}{\partial T_{0}}- \\
& -2 \Delta \hat{\phi}_{0} \frac{\partial \Delta \hat{\theta}_{1}}{\partial T_{0}}-2 \Delta \hat{\phi}_{0} \frac{\partial \Delta \hat{\theta}_{0}}{\partial T_{1}}-2 \frac{\partial \Delta \hat{\phi}_{0}}{\partial T_{0}} \frac{\partial \Delta \hat{r}_{0}}{\partial T_{1}}- \\
& -2 \frac{\partial \Delta \hat{\phi}_{0}}{\partial T_{0}} \frac{\partial \Delta \hat{r}_{1}}{\partial T_{0}}-2 \frac{\partial \Delta \hat{\phi}_{0}}{\partial T_{1}} \frac{\partial \Delta \hat{r}_{0}}{\partial T_{0}}-2 \frac{\partial \Delta \hat{\phi}_{1}}{\partial T_{0}} \frac{\partial \Delta \hat{r}_{0}}{\partial T_{0}}
\end{aligned}
$$

Expanding terms,

$$
\begin{aligned}
\frac{\partial^{2} \Delta \hat{\phi}_{1}}{\partial T_{0} \partial T_{1}}= & (9 A B D+3 \bar{A} B \bar{D}) \mathrm{e}^{i T_{0}}+(3 A B D+9 \bar{A} B \bar{D}) \mathrm{e}^{-i T_{0}}- \\
& -12 A B D \mathrm{e}^{2 i T_{0}}-12 \bar{A} B \bar{D} \mathrm{e}^{-2 i T_{0}} \\
\frac{\partial^{2} \Delta \hat{\phi}_{0}}{\partial T_{0} \partial T_{2}}= & i \frac{\partial D}{\partial T_{2}} \mathrm{e}^{i T_{0}}-i \frac{\partial D}{\partial T_{2}} \mathrm{e}^{-i T_{0}} \\
\frac{\partial^{2} \Delta \hat{\phi}_{0}}{\partial T_{1}^{2}}= & -\frac{9}{4} B^{2} D \mathrm{e}^{i T_{0}}-\frac{9}{4} B^{2} \bar{D} \mathrm{e}^{-i T_{0}} \\
\Delta \hat{\phi}_{1} \frac{\partial \Delta \hat{\theta}_{0}}{\partial T_{0}}= & \left(\frac{3}{2} B D \bar{A}-4 A^{2} \bar{D}+\frac{3}{2} A B \bar{D}+\frac{3}{2} B \bar{A} \bar{D}-\frac{9}{2} A B D+\frac{3}{4} i B \Delta \hat{\omega}_{\phi_{10}}\right) \mathrm{e}^{i T_{0}}+ \\
& +\left(\frac{3}{2} A B D+\frac{3}{2} B D \bar{A}-4 D \bar{A}^{2}+\frac{3}{2} A B \bar{D}-\frac{9}{2} B \bar{A} \bar{D}-\frac{3}{4} i B \Delta \hat{\omega}_{\phi_{10}}\right) \mathrm{e}^{-i T_{0}}+ \\
& +\left(3 A B D+2 A \bar{A} D+2 A^{2} \bar{D}+2 A \bar{A} \bar{D}+i A \Delta \hat{\omega}_{\phi_{10}}-6 A^{2} D\right) \mathrm{e}^{2 i T_{0}}+ \\
& +\left(2 A D \bar{A}+2 D \bar{A}^{2}+2 A \bar{A} \bar{D}+3 \bar{A} \bar{D}-6 \bar{A}^{2} \bar{D}-i \Delta \hat{\omega}_{\phi_{10}}\right) \mathrm{e}^{-2 i T_{0}}+ \\
& +4 A^{2} D \mathrm{e}^{3 i T_{0}}+4 \bar{A}^{2} \bar{D} \mathrm{e}^{-3 i T_{0}}+2 A^{2} D-i A \Delta \hat{\omega}_{\phi_{10}}-4 A \bar{A} D-3 B D \bar{A}+ \\
& +i \bar{A} \Delta \hat{\omega}_{\phi_{10}}+2 D \bar{A}^{2}+2 A^{2} \bar{D}-3 A B \bar{D}-4 A \bar{A} \bar{D}+2 \bar{A}^{2} \bar{D}
\end{aligned}
$$




$$
\begin{aligned}
\Delta \hat{\phi}_{0} \frac{\partial \Delta \hat{\theta}_{1}}{\partial T_{0}}= & \left(5 A^{2} \bar{D}+D^{2} \bar{D}\right) \mathrm{e}^{i T_{0}}+\left(5 \bar{A}^{2} D+\bar{D}^{2} D\right) \mathrm{e}^{-i T_{0}}+ \\
& +\left(A B D-\frac{9}{2} A^{2} D-\frac{1}{2} D^{3}-\bar{A} D B-\frac{1}{2} D \bar{A}^{2}-\frac{1}{2} D \bar{D}^{2}+i D \Delta \hat{v}_{r_{10}}+\right. \\
& \left.+\frac{1}{2} D \Delta \hat{\omega}_{\theta_{10}}\right) \mathrm{e}^{2 i T_{0}}+\left(\bar{A} \bar{D} B-\frac{1}{2} A^{2} \bar{D}-\bar{A} B D-\frac{1}{2} D^{2} \bar{D}-\frac{9}{2} \bar{A}^{2} \bar{D}-\frac{1}{2} \bar{D}^{3}-\right. \\
& \left.-i \bar{D} \Delta \hat{v}_{r_{10}}+\frac{1}{2} \bar{D} \Delta \hat{\omega}_{\theta_{10}}\right) \mathrm{e}^{-2 i T_{0}}+\left(5 A^{2} D+D^{3}\right) \mathrm{e}^{3 i T_{0}}+ \\
& +\left(5 \bar{A}^{2} \bar{D}+\bar{D}^{3}\right) \mathrm{e}^{-3 i T_{0}}-\frac{1}{2} A^{2} D-\frac{5}{2} A B D-\frac{1}{2} D^{3}+\frac{5}{2} B \bar{A} D-\frac{9}{2} \bar{A}^{2} D- \\
& -\frac{9}{2} A^{2} \bar{D}+A B \bar{D}-\frac{1}{2} D^{2} \bar{D}-B \bar{A} \bar{D}-\frac{1}{2} \bar{A}^{2} \bar{D}-\frac{1}{2} D \bar{D}^{2}-\frac{1}{2} \bar{D}^{3}-i D \Delta \hat{v}_{r_{10}}+ \\
& +i \bar{D} \Delta \hat{v}_{r_{10}}+\frac{1}{2} D \Delta \hat{\omega}_{\theta_{10}}+\frac{1}{2} \bar{D} \Delta \hat{\omega}_{\theta_{10}} \\
\Delta \hat{\phi}_{0} \frac{\partial \Delta \hat{\theta}_{0}}{\partial T_{1}}= & \frac{9}{4} A^{2} D+\frac{3}{2} A B D+\frac{15}{8} B^{2} D+\frac{3}{4} D^{3}+3 A D \bar{A}+\frac{3}{2} B D \bar{A}+\frac{9}{4} D \bar{A}^{2}+\frac{3}{4} D \bar{D}^{2}- \\
& \left.-\frac{3}{4} D \Delta \hat{\omega}_{\theta_{10}}\right) \mathrm{e}^{i T_{0}}+\left(\frac{9}{4} A^{2} \bar{D}+\frac{3}{2} A B \bar{D}+\frac{15}{8} B^{2} \bar{D}+\frac{3}{4} D^{2} \bar{D}+3 A \bar{A} \bar{D}+\right. \\
& \left.+\frac{3}{2} B \bar{A} \bar{D}+\frac{9}{4} \bar{A}^{2} \bar{D}+\frac{3}{4} \bar{D}^{3}-\frac{3}{4} \bar{D} \Delta \hat{\omega}_{\theta_{10}}\right) \mathrm{e}^{-i T_{0}}+3 A B D \mathrm{e}^{2 i T_{0}}+3 \bar{A} B \bar{D} \mathrm{e}^{-2 i T_{0}}+ \\
& +3 B D \bar{A}+3 A B \bar{D}
\end{aligned}
$$

$$
\begin{aligned}
\frac{\partial \Delta \hat{\phi}_{0}}{\partial T_{0}} \frac{\partial \Delta \hat{r}_{0}}{\partial T_{1}}= & \frac{3}{2} A B D \mathrm{e}^{2 i T_{0}}+\frac{3}{2} A B \bar{D} \mathrm{e}^{-2 i T_{0}}-\frac{3}{2} B D \bar{A}-\frac{3}{2} A B \bar{D} \\
\frac{\partial \Delta \hat{\phi}_{0}}{\partial T_{0}} \frac{\partial \Delta \hat{r}_{1}}{\partial T_{0}}= & -2 A^{2} \bar{D} \mathrm{e}^{i T_{0}}-2 \bar{A}^{2} D \mathrm{e}^{-i T_{0}}+\left(\frac{1}{4} D \Delta \hat{\omega}_{\theta_{10}}+\frac{1}{2} i \Delta \hat{v}_{r_{10}}-\frac{9}{4} A^{2} D-\frac{1}{2} A B D-\right. \\
& \left.-\frac{1}{4} D^{3}-\frac{1}{2} \bar{A} B D-\frac{1}{4} \bar{A}^{2} D-\frac{1}{4} D \bar{D}^{2}\right) \mathrm{e}^{2 i T_{0}}+\left(\frac{1}{4} \bar{D} \Delta \hat{\omega}_{\theta_{10}}-\frac{1}{2} i \bar{D} \Delta \hat{v}_{r_{10}}-\right. \\
& \left.-\frac{1}{4} A^{2} \bar{D}-\frac{1}{2} A B \bar{D}-\frac{1}{4} D^{2} \bar{D}-\frac{1}{2} \bar{A} B \bar{D}-\frac{9}{4} \bar{A}^{2} \bar{D}-\frac{1}{4} \bar{D}^{3}\right) \mathrm{e}^{-2 i T_{0}}+ \\
& +2 A^{2} D \mathrm{e}^{3 i T_{0}}+2 \bar{A}^{2} \bar{D} \mathrm{e}^{-3 i T_{0}}+\frac{1}{4} A^{2} D+\frac{1}{2} A B D+\frac{1}{4} D^{3}+\frac{1}{2} \bar{A} B D+ \\
& +\frac{9}{4} \bar{A}^{2} D+\frac{9}{4} A^{2} \bar{D}+\frac{1}{2} A B \bar{D}+\frac{1}{4} D^{2} \bar{D}+\frac{1}{2} \bar{A} B D+\frac{1}{4} \bar{A}^{2} \bar{D}+\frac{1}{4} D \bar{D}^{2}+ \\
& +\frac{1}{4} \bar{D}^{3}+\frac{1}{2} i D \Delta \hat{v}_{r_{10}}-\frac{1}{2} i \bar{D} \Delta \hat{v}_{r_{10}}-\frac{1}{4} D \Delta \hat{\omega}_{\theta_{10}}-\frac{1}{4} \bar{D} \Delta \hat{\omega}_{\theta_{10}}
\end{aligned}
$$

$\frac{\partial \Delta \hat{\phi}_{0}}{\partial T_{1}} \frac{\partial \Delta \hat{r}_{0}}{\partial T_{0}}=\frac{3}{2} A B D \mathrm{e}^{2 i T_{0}}+\frac{3}{2} \bar{A} B \bar{D} \mathrm{e}^{-2 i T_{0}}-\frac{3}{2} \bar{A} B D-\frac{3}{2} A B \bar{D}$

$\frac{\partial \Delta \hat{\phi}_{1}}{\partial T_{0}} \frac{\partial \Delta \hat{r}_{0}}{\partial T_{0}}=-4 A \bar{A} D \mathrm{e}^{i T_{0}}-4 \bar{A} A \bar{D} \mathrm{e}^{-i T_{0}}+$ 


$$
\begin{aligned}
& +\left(A \bar{A} D+A^{2} \bar{D}+A \bar{A} \bar{D}-3 A^{2} D+\frac{1}{2} i A \Delta \hat{\omega}_{\phi_{10}}\right) \mathrm{e}^{2 i T_{0}}+ \\
& +\left(A \bar{A} D+\bar{A}^{2} D+A \bar{A} \bar{D}-3 \bar{A}^{2} \bar{D}-\frac{1}{2} i \hat{A} \Delta \hat{\omega}_{\phi_{10}}\right) \mathrm{e}^{-2 i T_{0}}+ \\
& +4 A^{2} D \mathrm{e}^{3 i T_{0}}+4 \bar{A}^{2} \bar{D} \mathrm{e}^{-3 i T_{0}}-A^{2} D+2 A \bar{A} D-\bar{A}^{2} D-A^{2} \bar{D}+ \\
& +2 A \bar{A} \bar{D}+\frac{1}{2} i A \Delta \hat{\omega}_{\phi_{10}}-\frac{1}{2} \Delta \hat{\omega}_{\phi_{10}}
\end{aligned}
$$

Collecting these terms together leads to the result for $\gamma_{\phi_{2}}, \eta_{\phi_{2}}, \chi_{\phi_{2}}$ and $\sigma_{\phi_{2}}$ in chapter (4). 


\section{Appendix C}

\section{Plots}



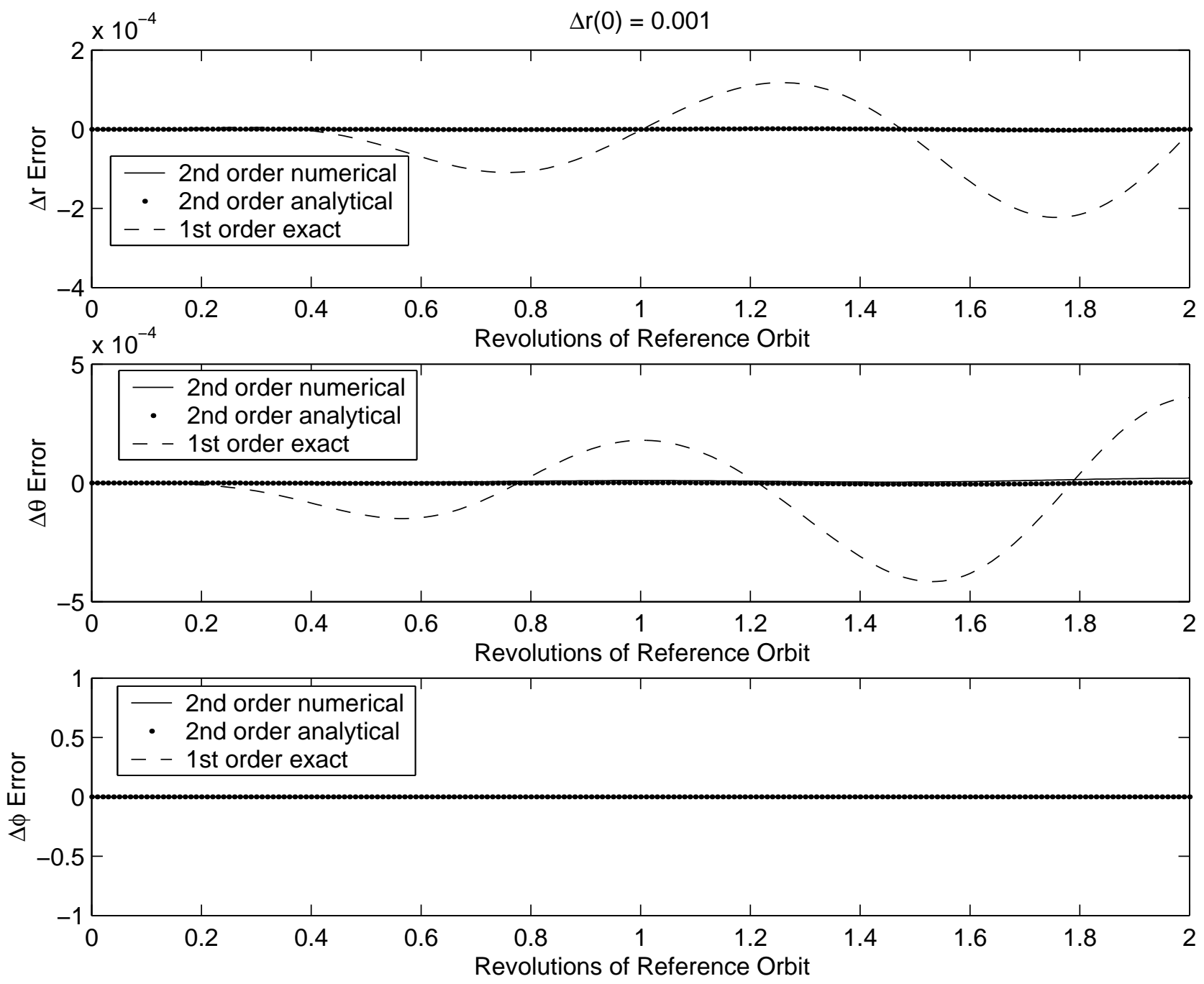

Figure C.1: Error of Position Variables for $\Delta \hat{r}(0)=0.001$ 

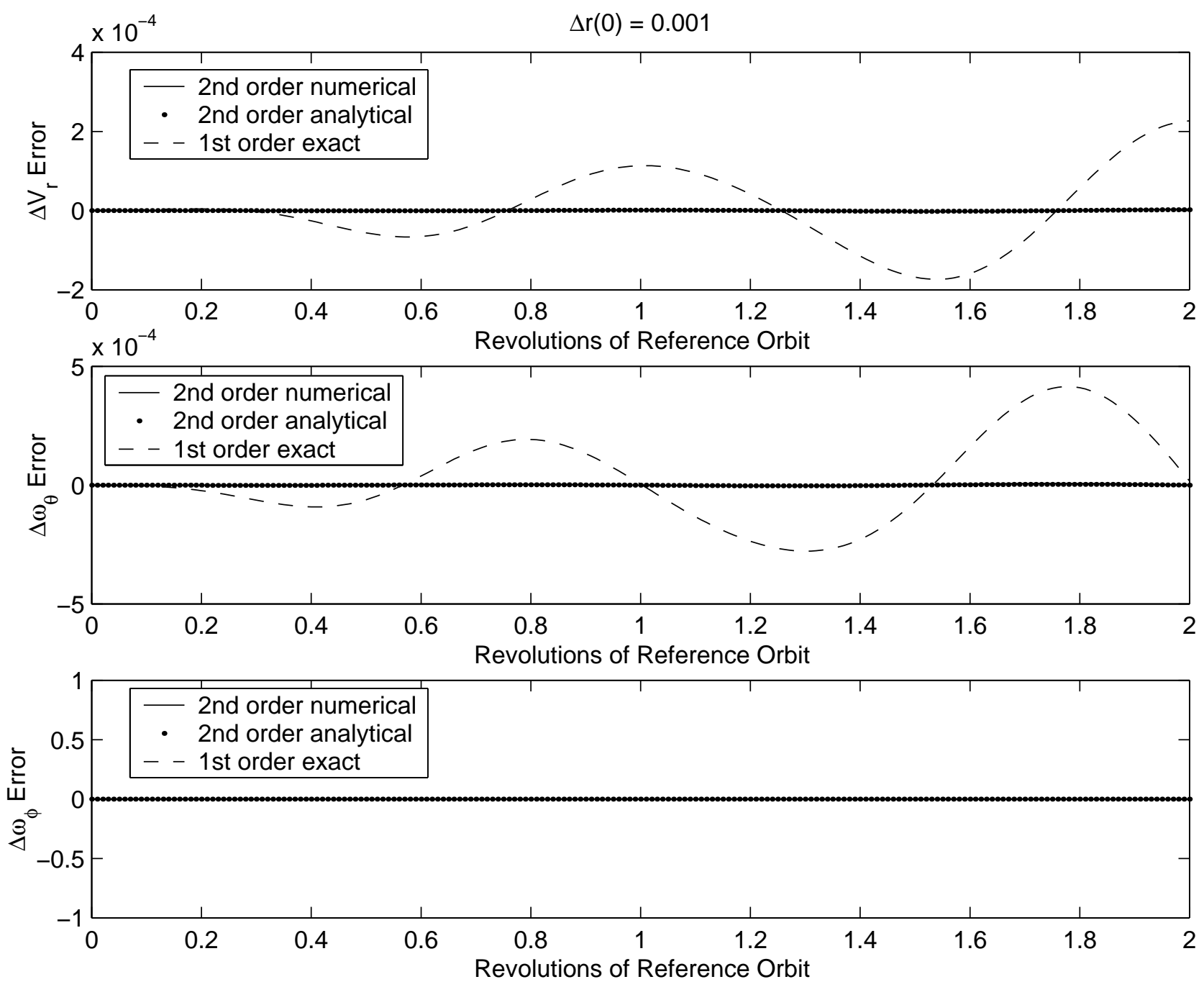

Figure C.2: Error of Velocity Variables for $\Delta \hat{r}(0)=0.001$ 

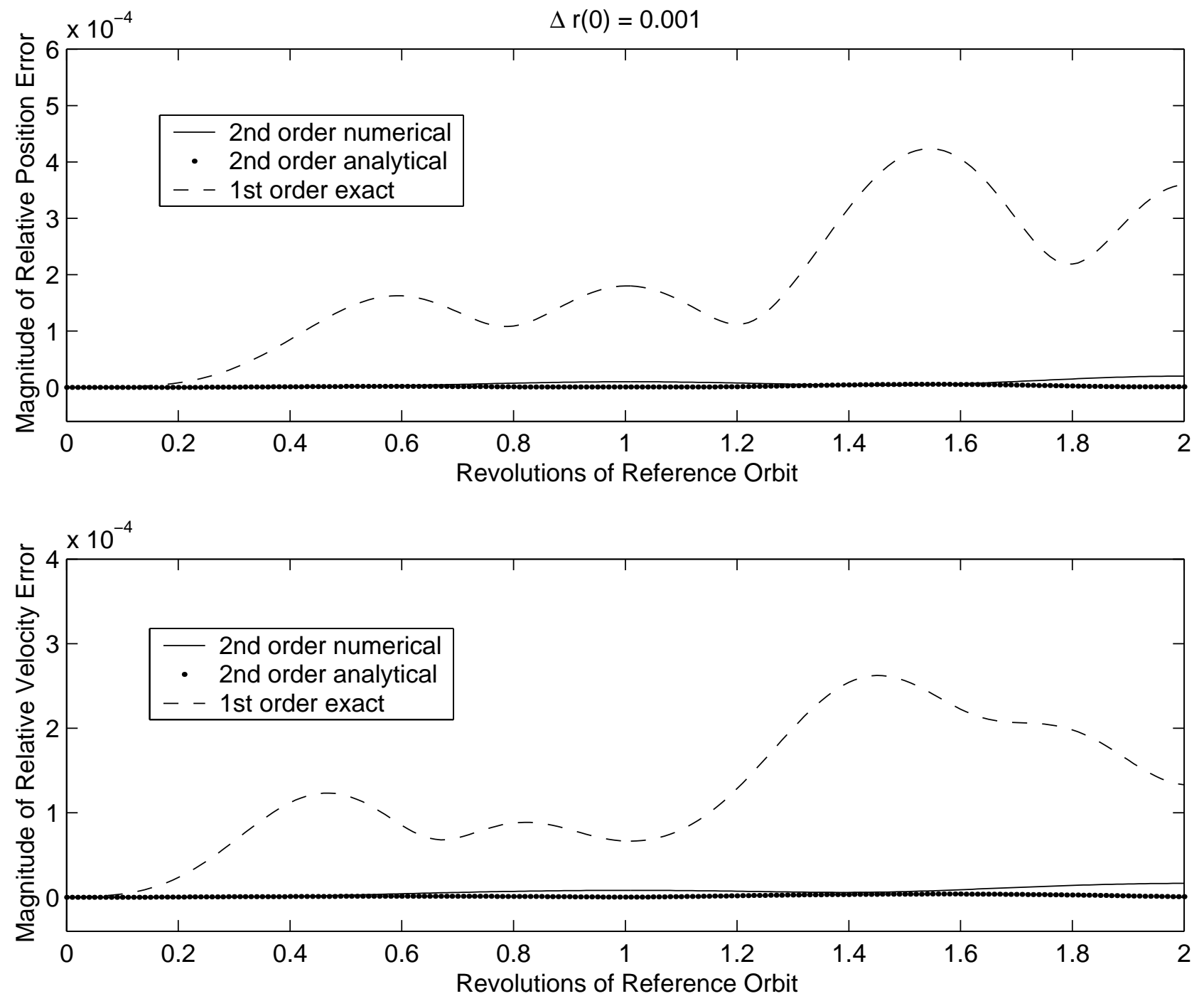

Figure C.3: Magnitude of Relative Position and Velocity Error for $\Delta \hat{r}(0)=0.001$ 

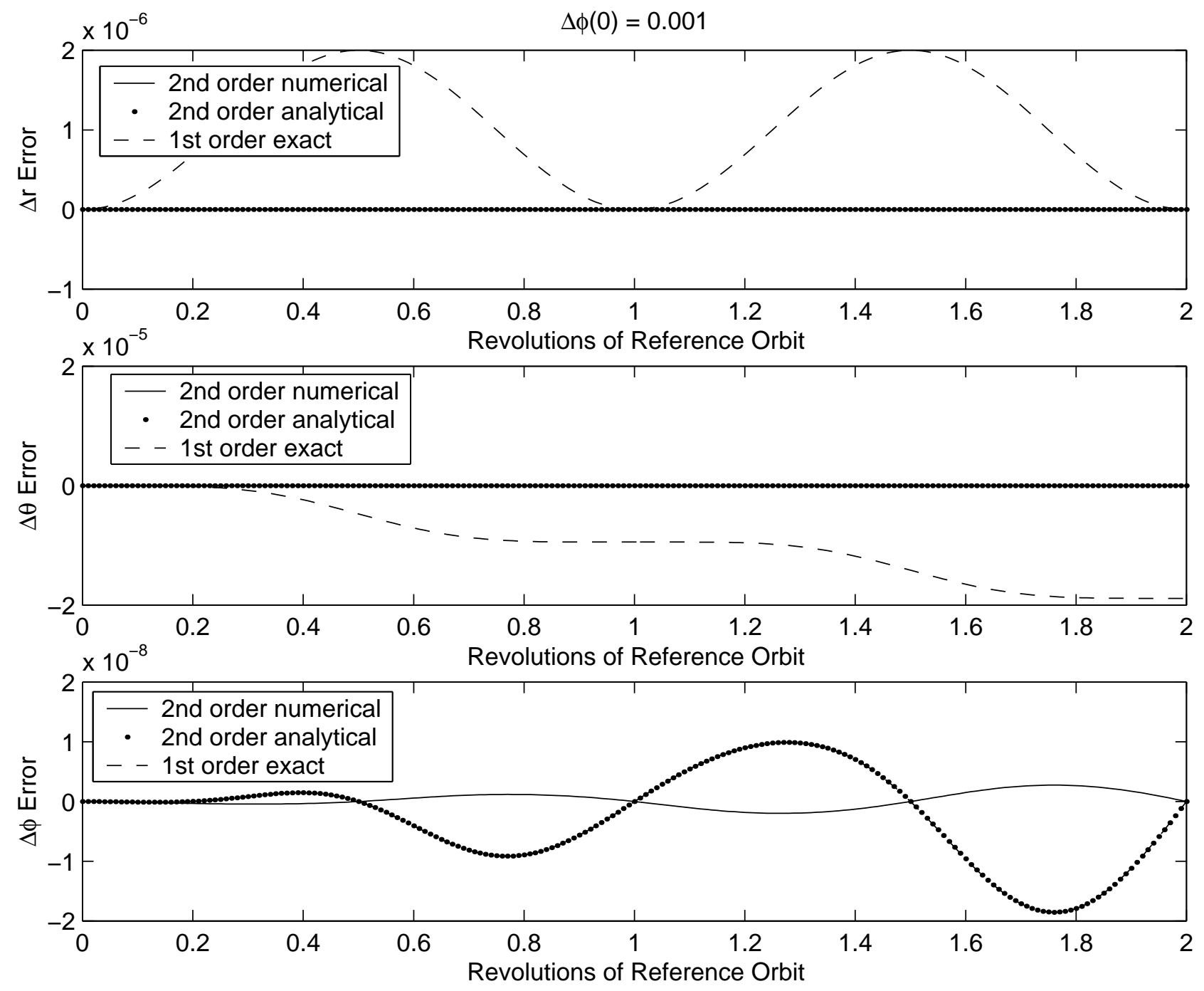

Figure C.4: Error of Position Variables for $\Delta \hat{\phi}(0)=0.001$ 

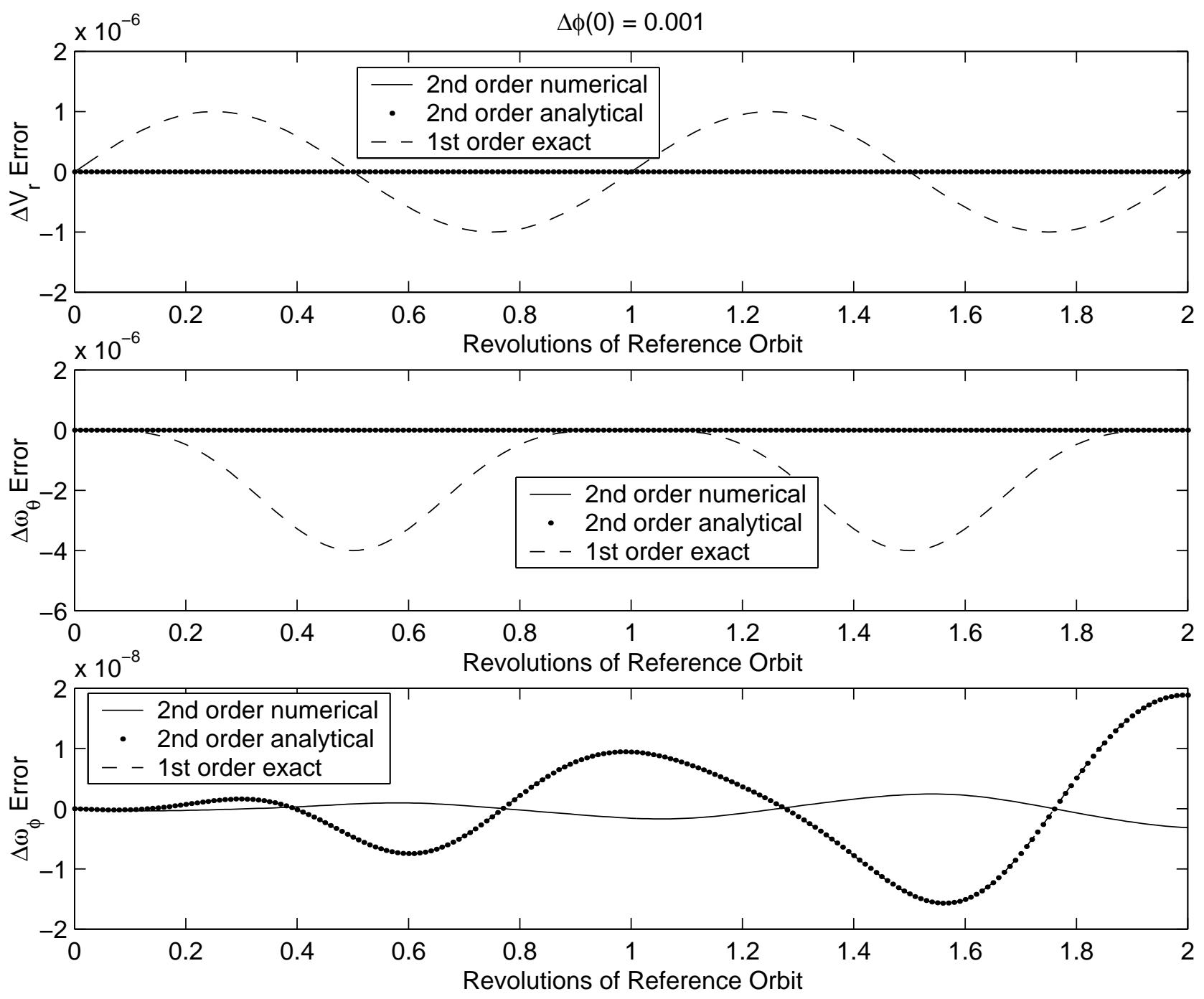

Figure C.5: Error of Velocity Variables for $\Delta \hat{\phi}(0)=0.001$ 

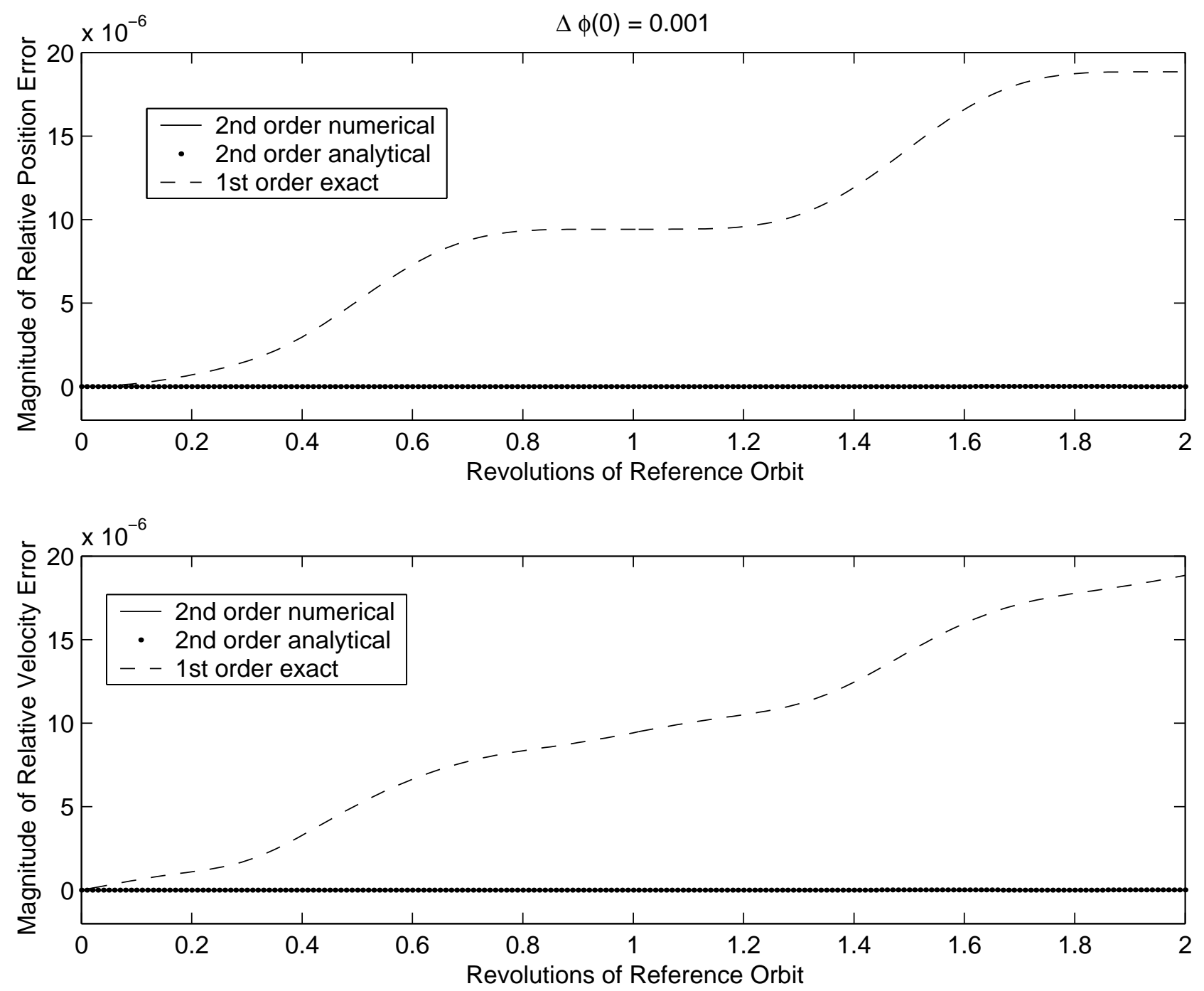

Figure C.6: Magnitude of Relative Position and Velocity Error for $\Delta \hat{\phi}(0)=0.001$ 

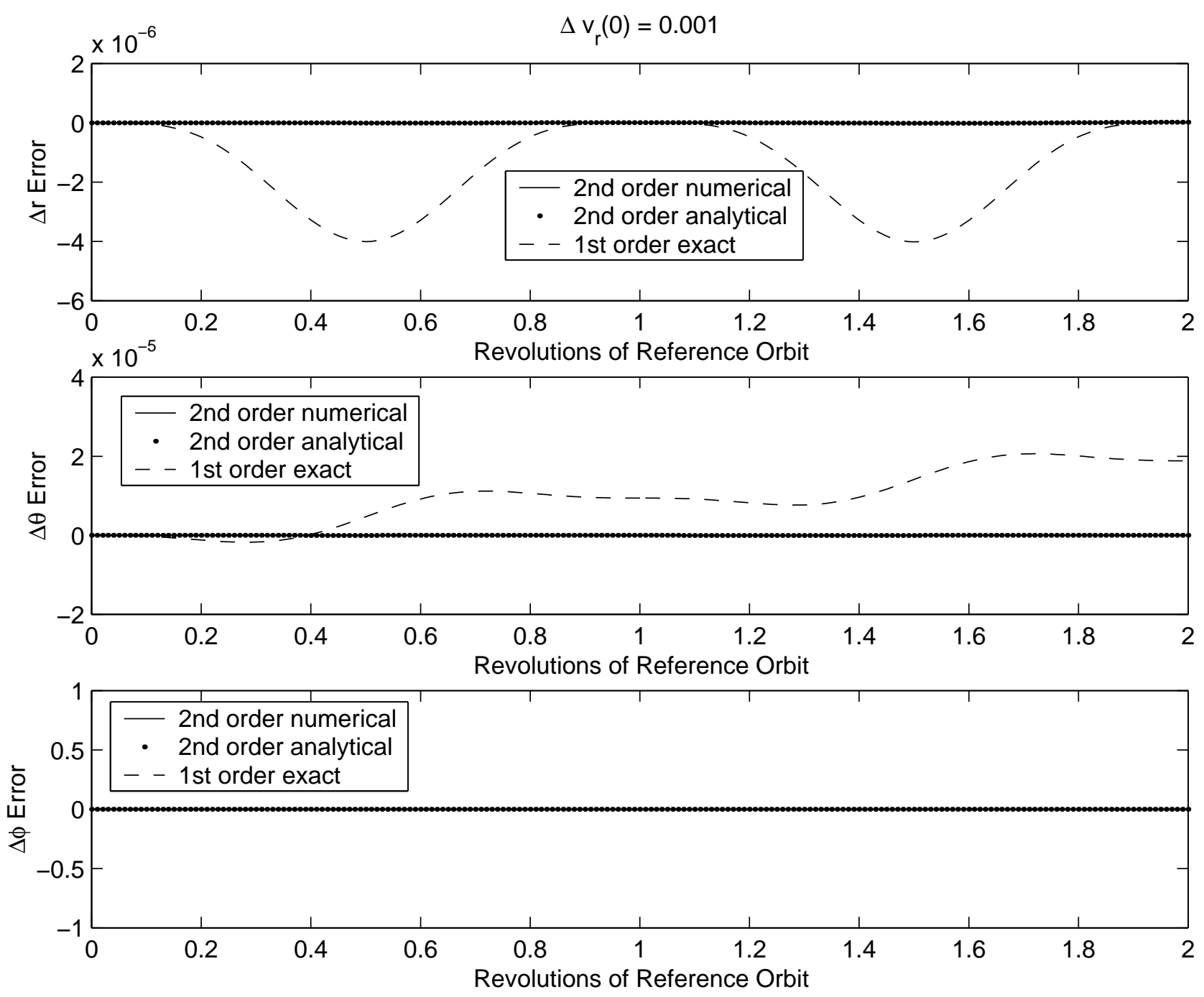

Figure C.7: Error of Position Variables for $\Delta \hat{v}_{r}(0)=0.001$ 

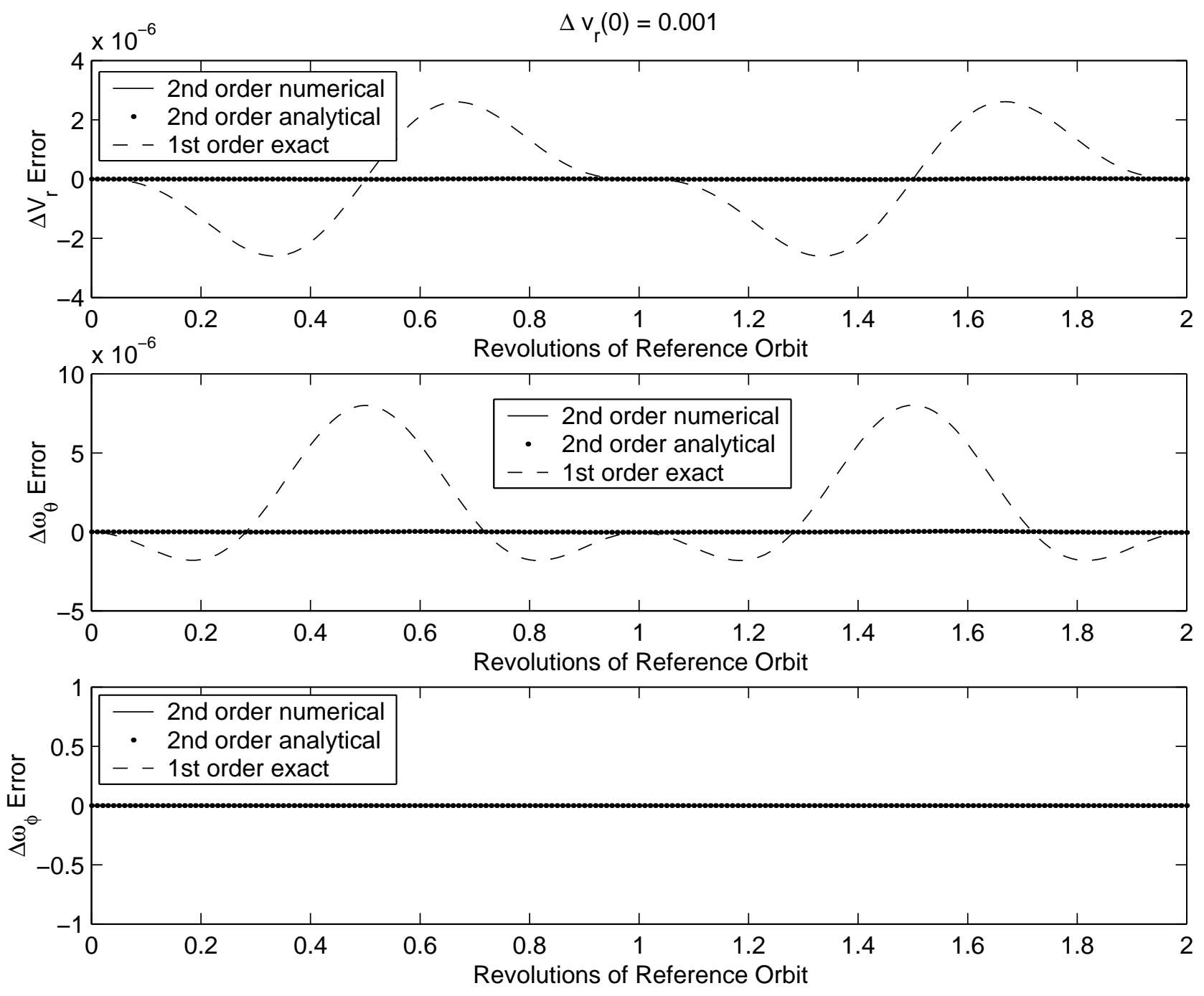

Figure C.8: Error of Velocity Variables for $\Delta \hat{v}_{r}(0)=0.001$ 

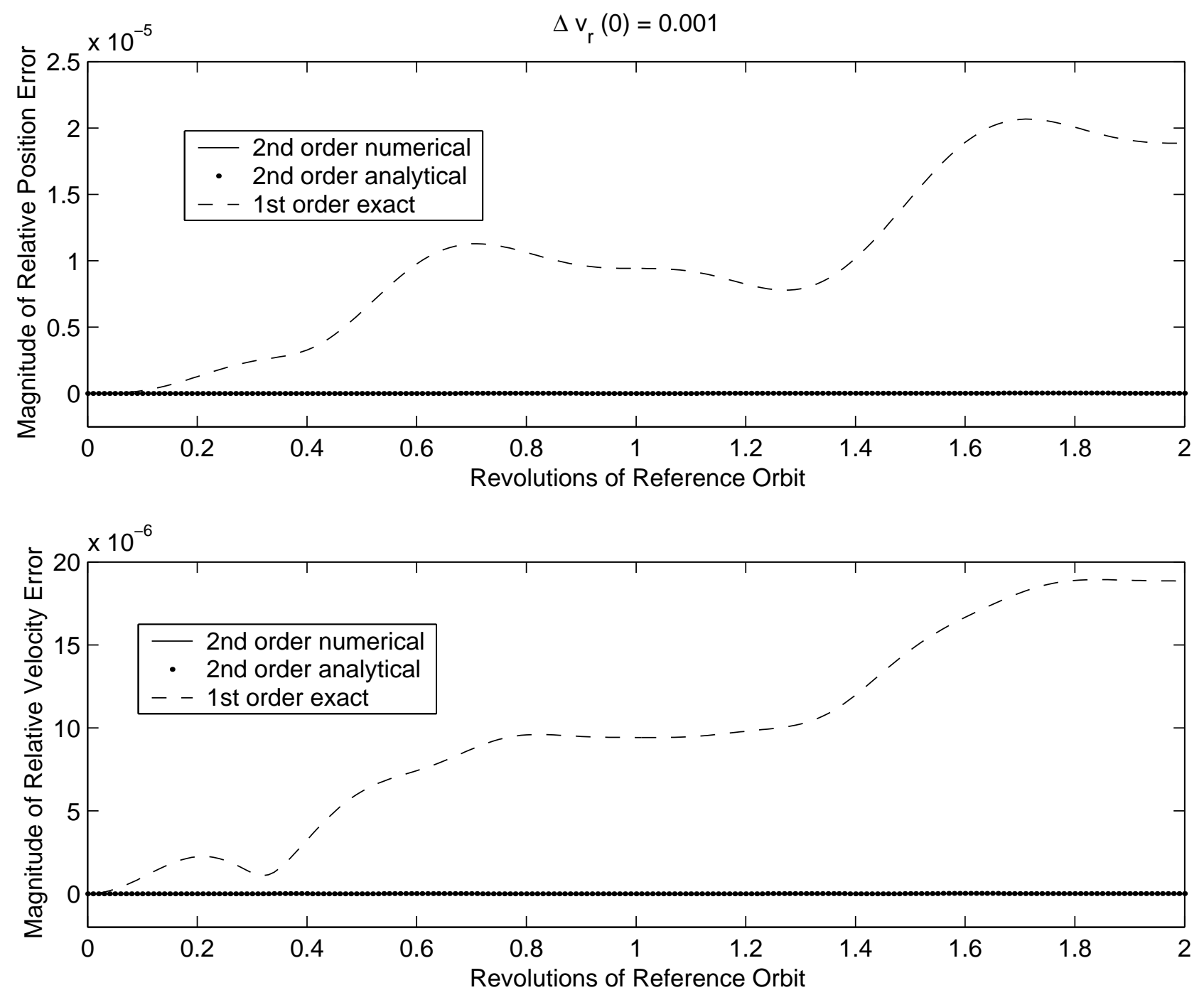

Figure C.9: Magnitude of Relative Position and Velocity Error for $\Delta \hat{v}_{r}(0)=0.001$ 

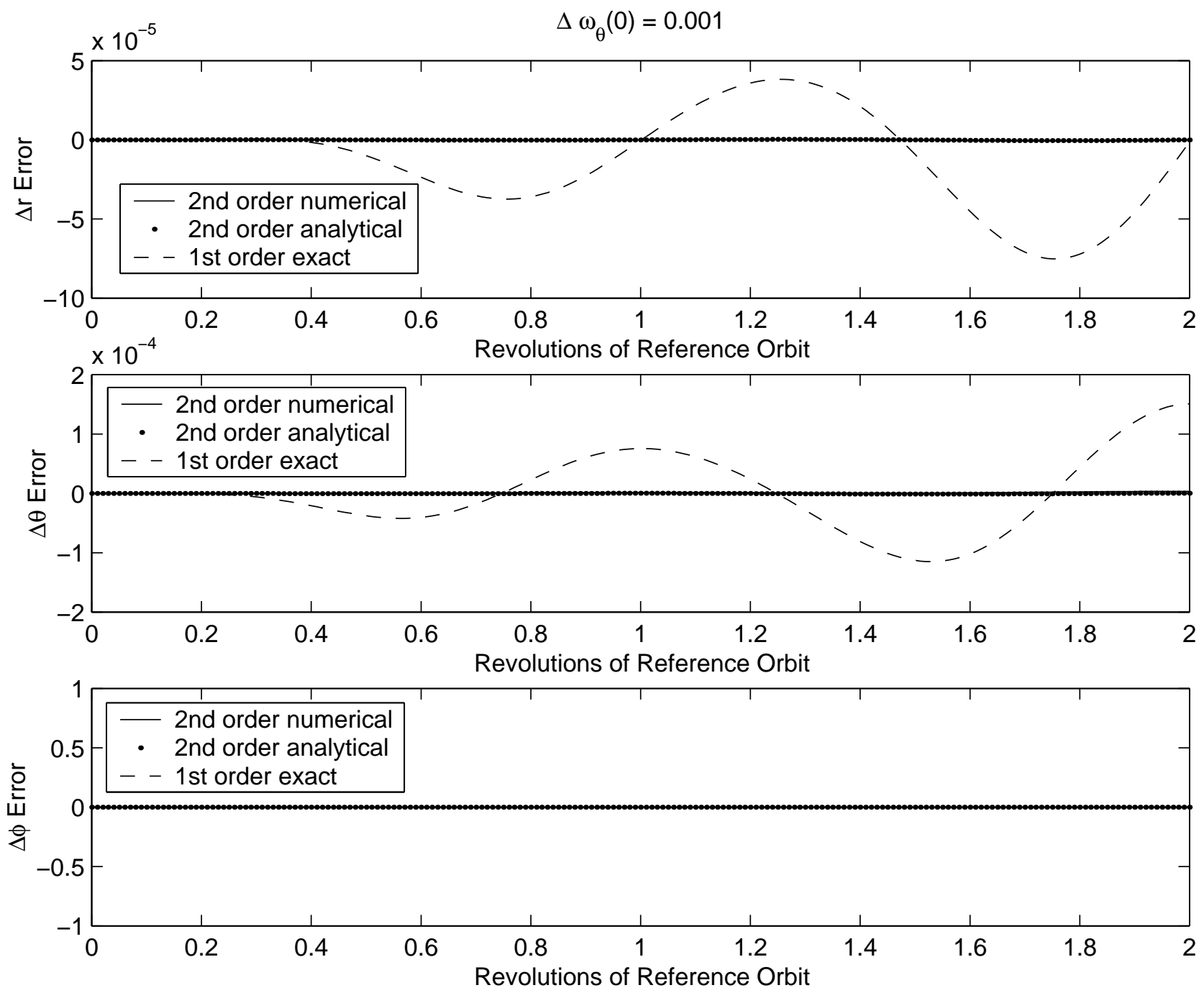

Figure C.10: Error of Position Variables for $\Delta \hat{\omega}_{\theta}(0)=0.001$ 

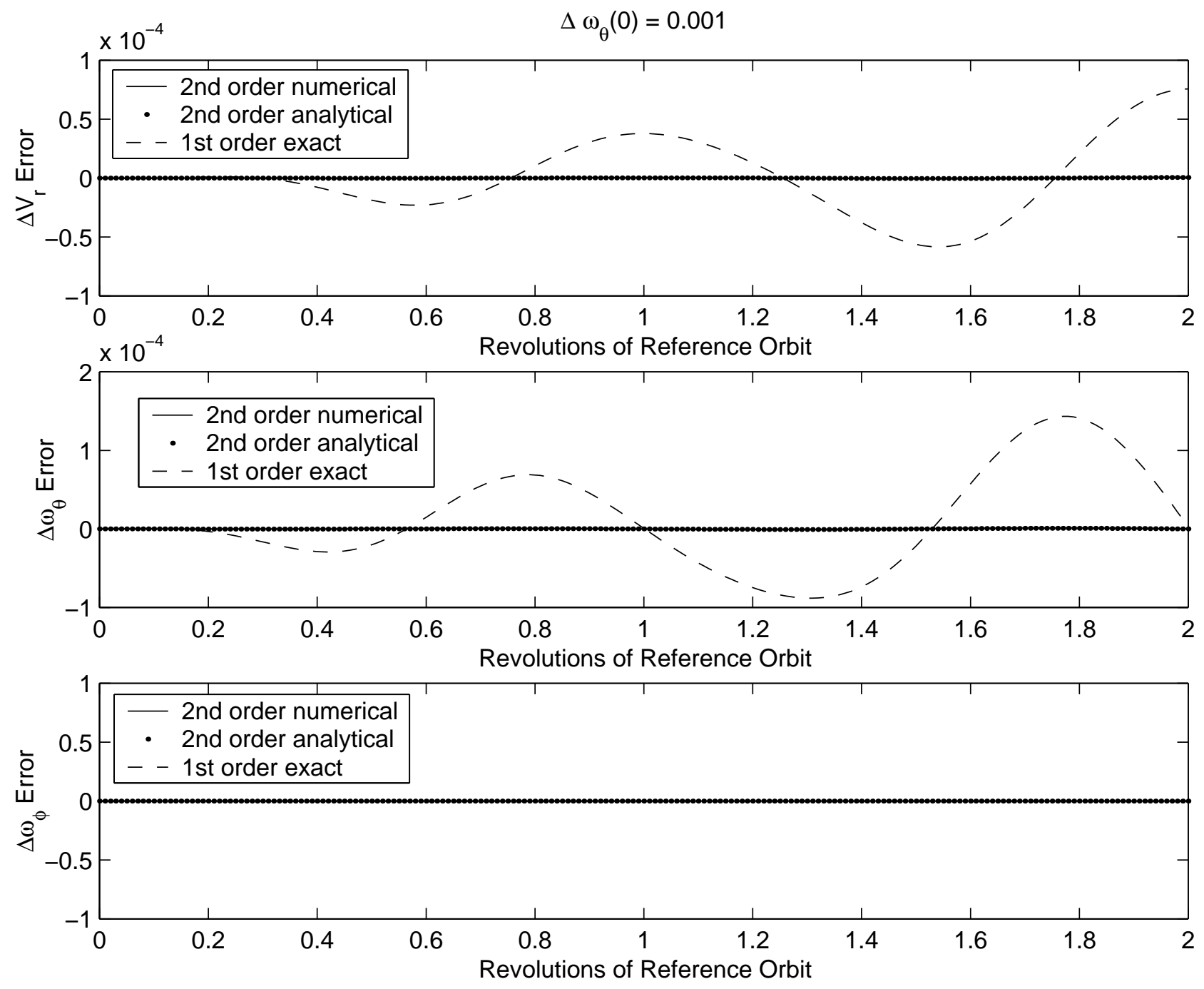

Figure C.11: Error of Velocity Variables for $\Delta \hat{\omega}_{\theta}(0)=0.001$ 

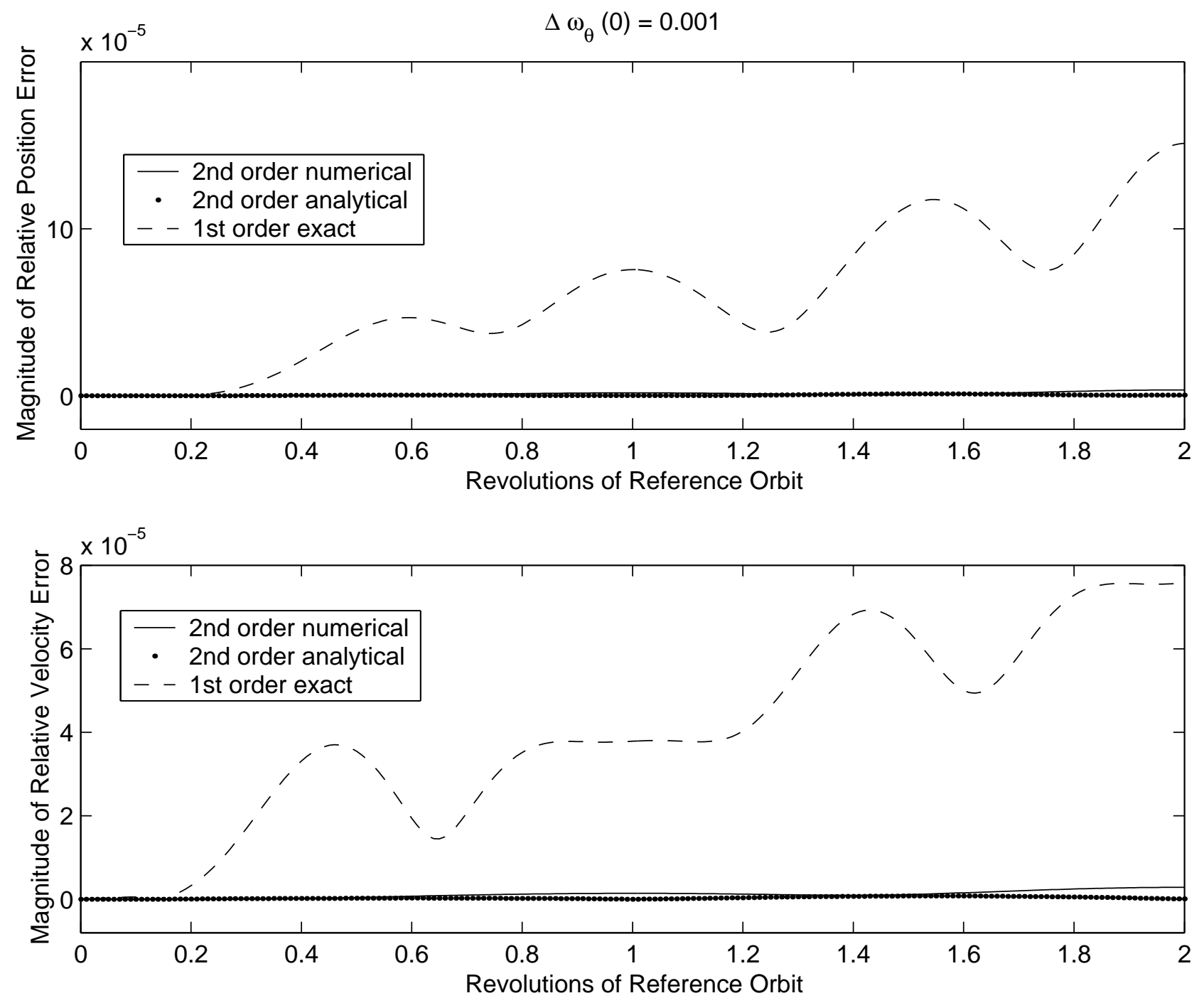

Figure C.12: Magnitude of Relative Position and Velocity Error for $\Delta \hat{\omega}_{\theta}(0)=0.001$ 

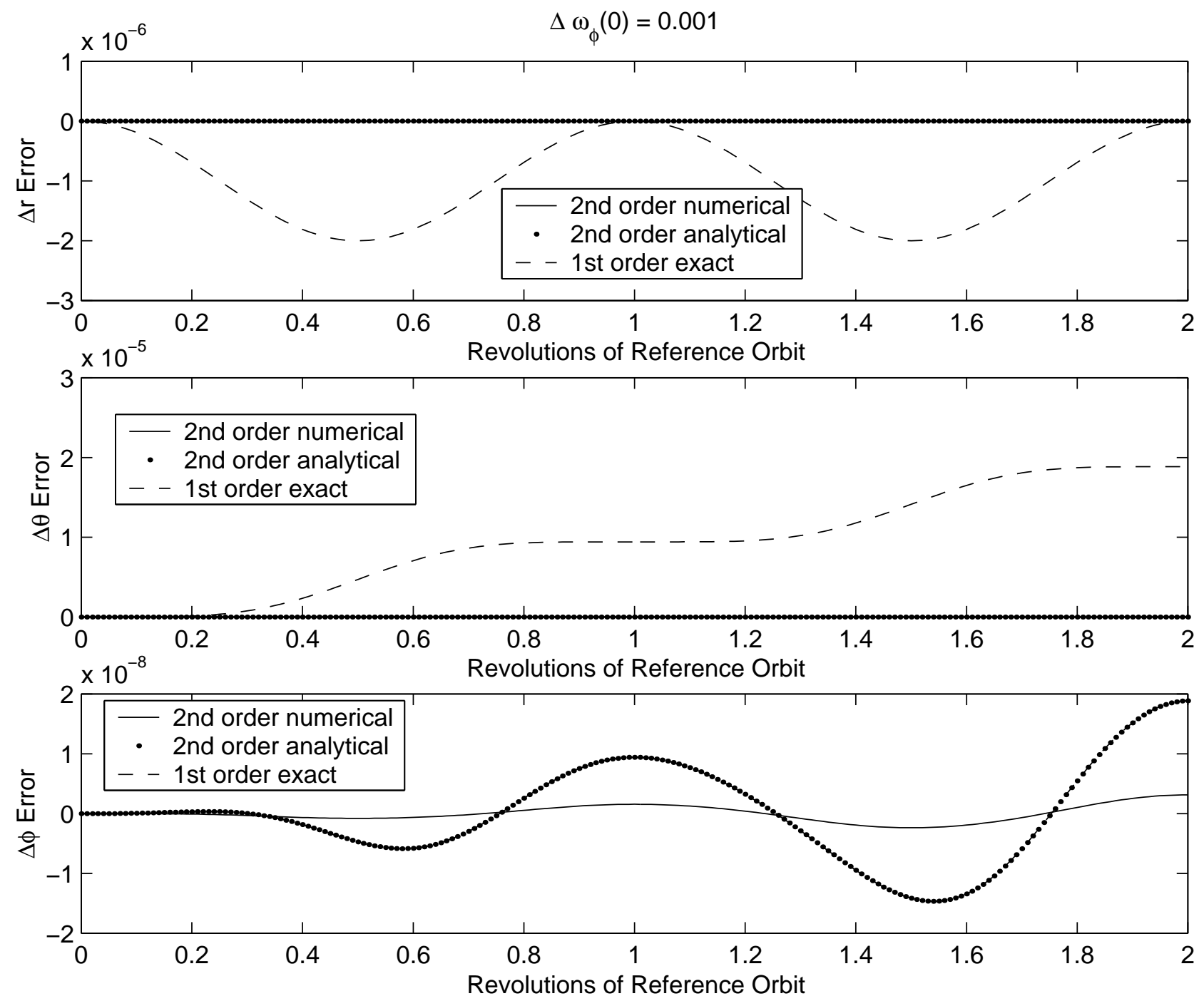

Figure C.13: Error of Position Variables for $\Delta \hat{\omega}_{\phi}(0)=0.001$ 

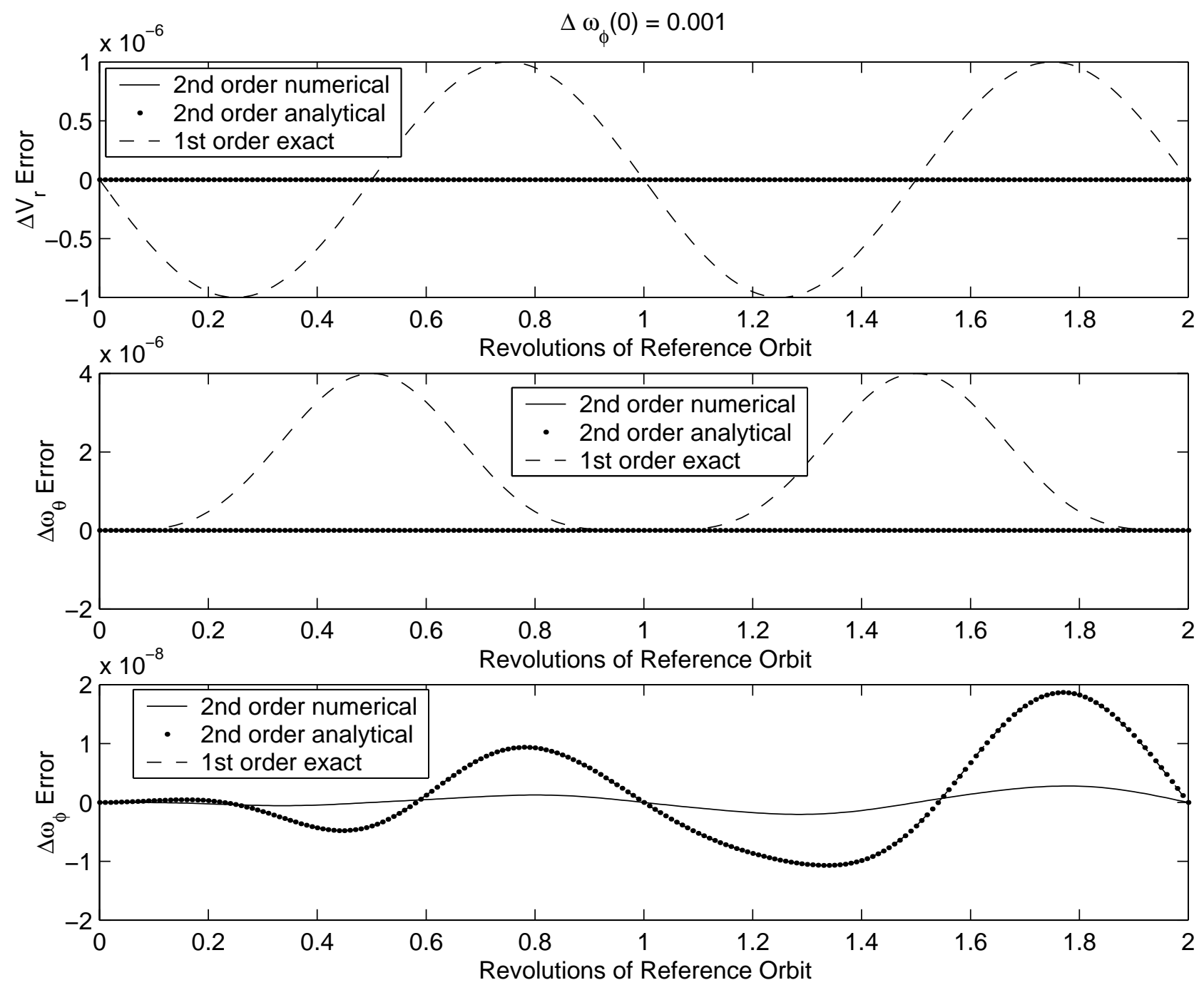

Figure C.14: Error of Velocity Variables for $\Delta \hat{\omega}_{\phi}(0)=0.001$ 

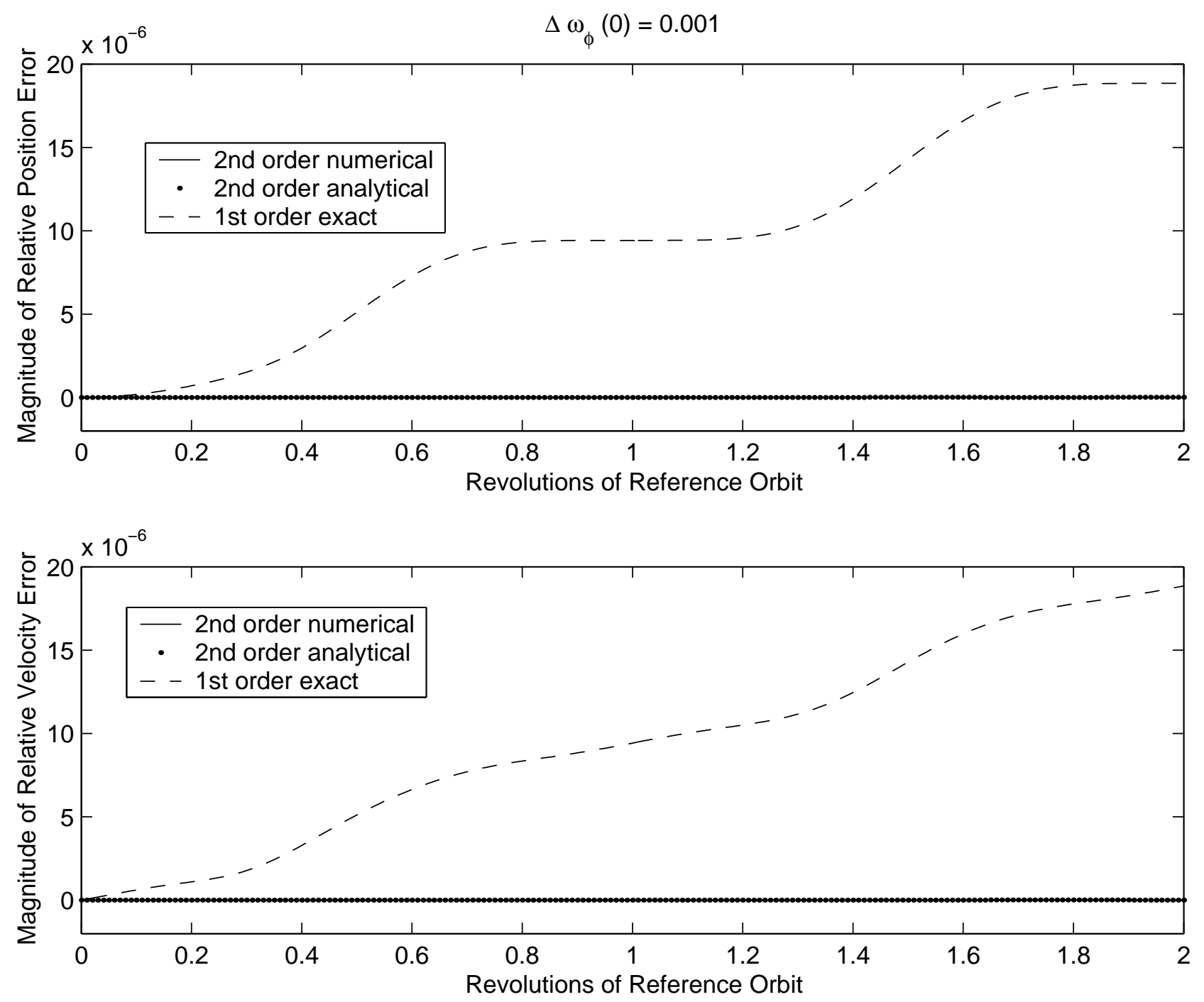

Figure C.15: Magnitude of Relative Position and Velocity Error for $\Delta \hat{\omega}_{\phi}(0)=0.001$ 

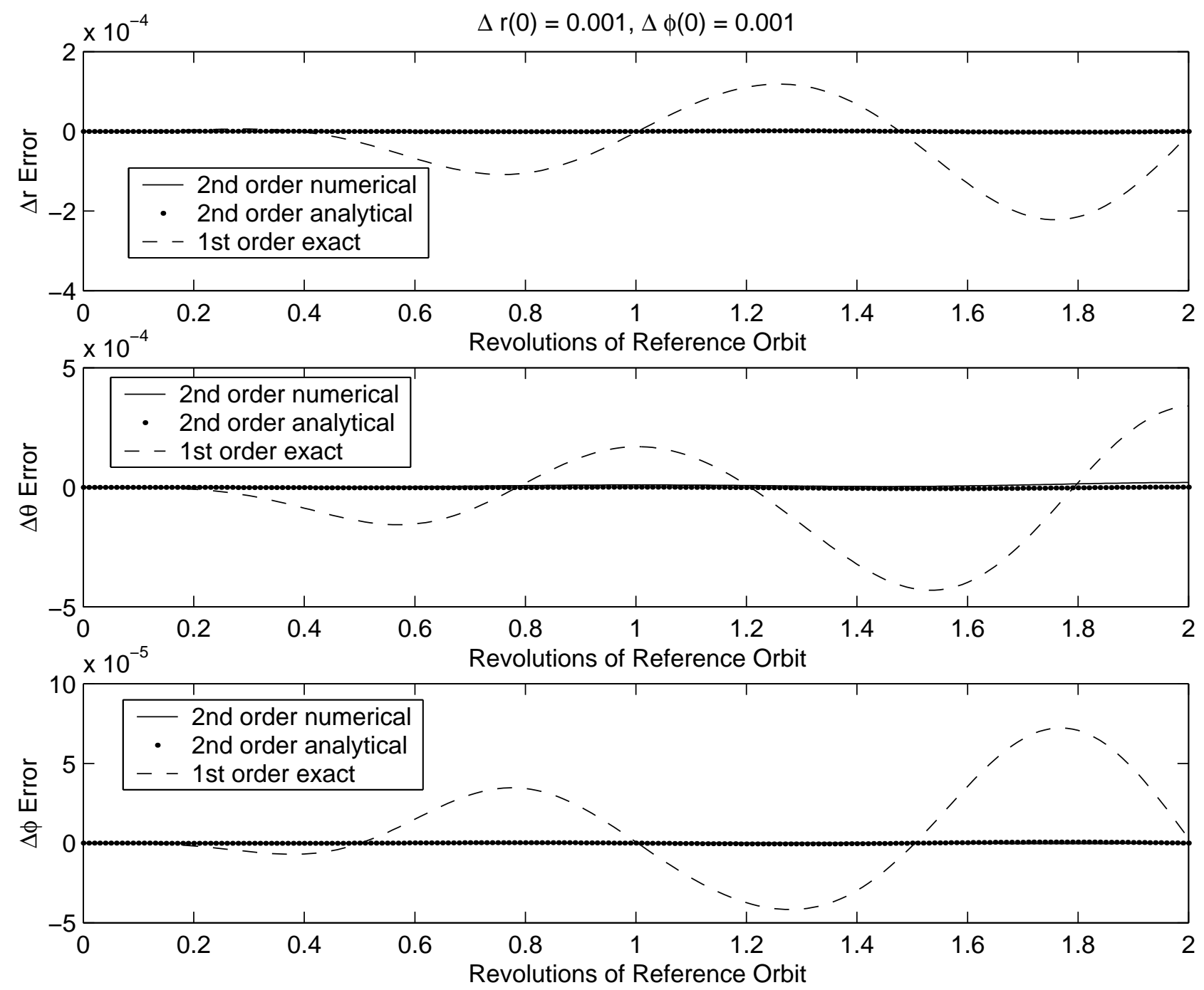

Figure C.16: Error of Position Variables for $\Delta \hat{r}(0)=0.001, \Delta \hat{\phi}(0)=0.001$ 

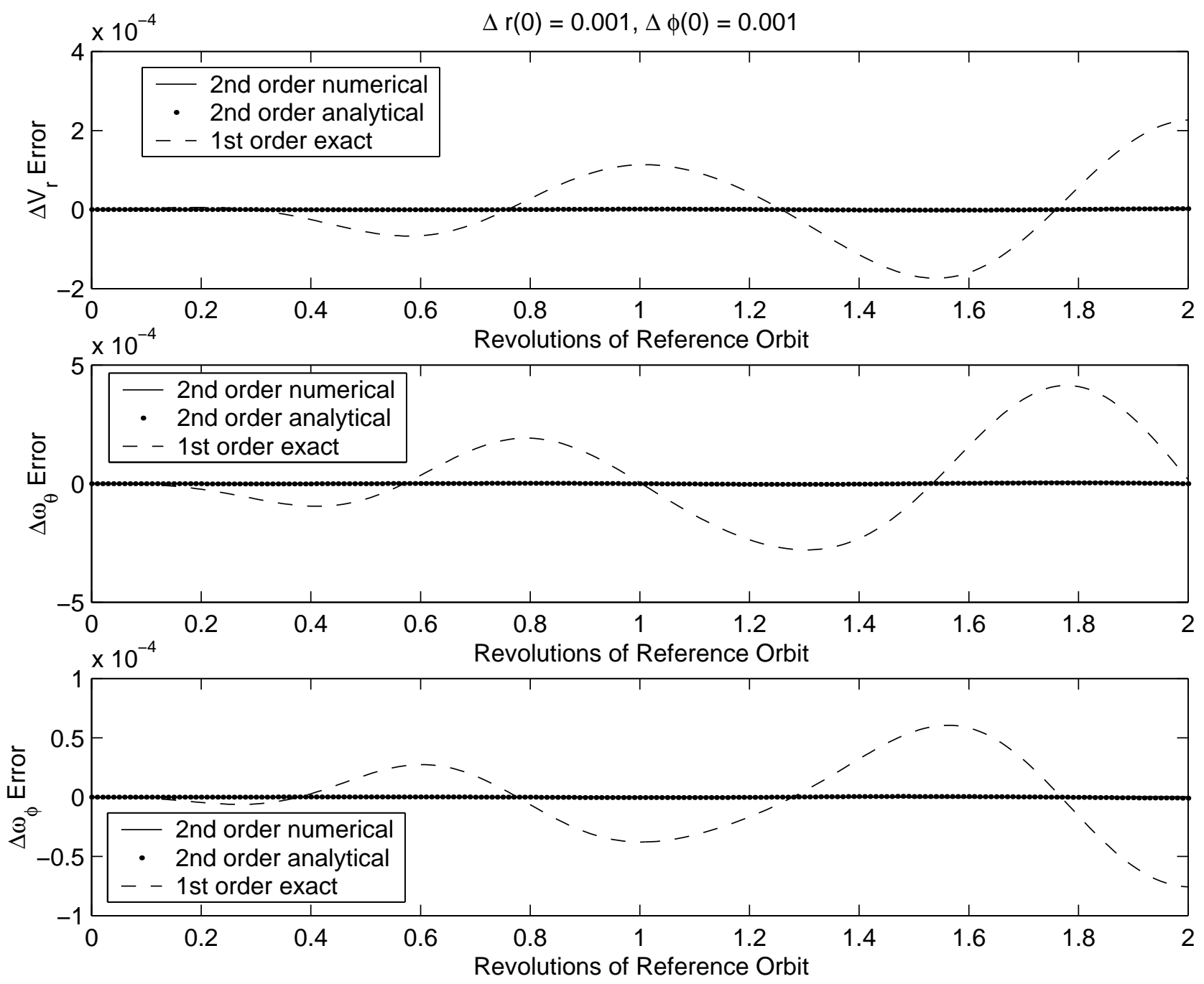

Figure C.17: Error of Velocity Variables for $\Delta \hat{r}(0)=0.001, \Delta \hat{\phi}(0)=0.001$ 

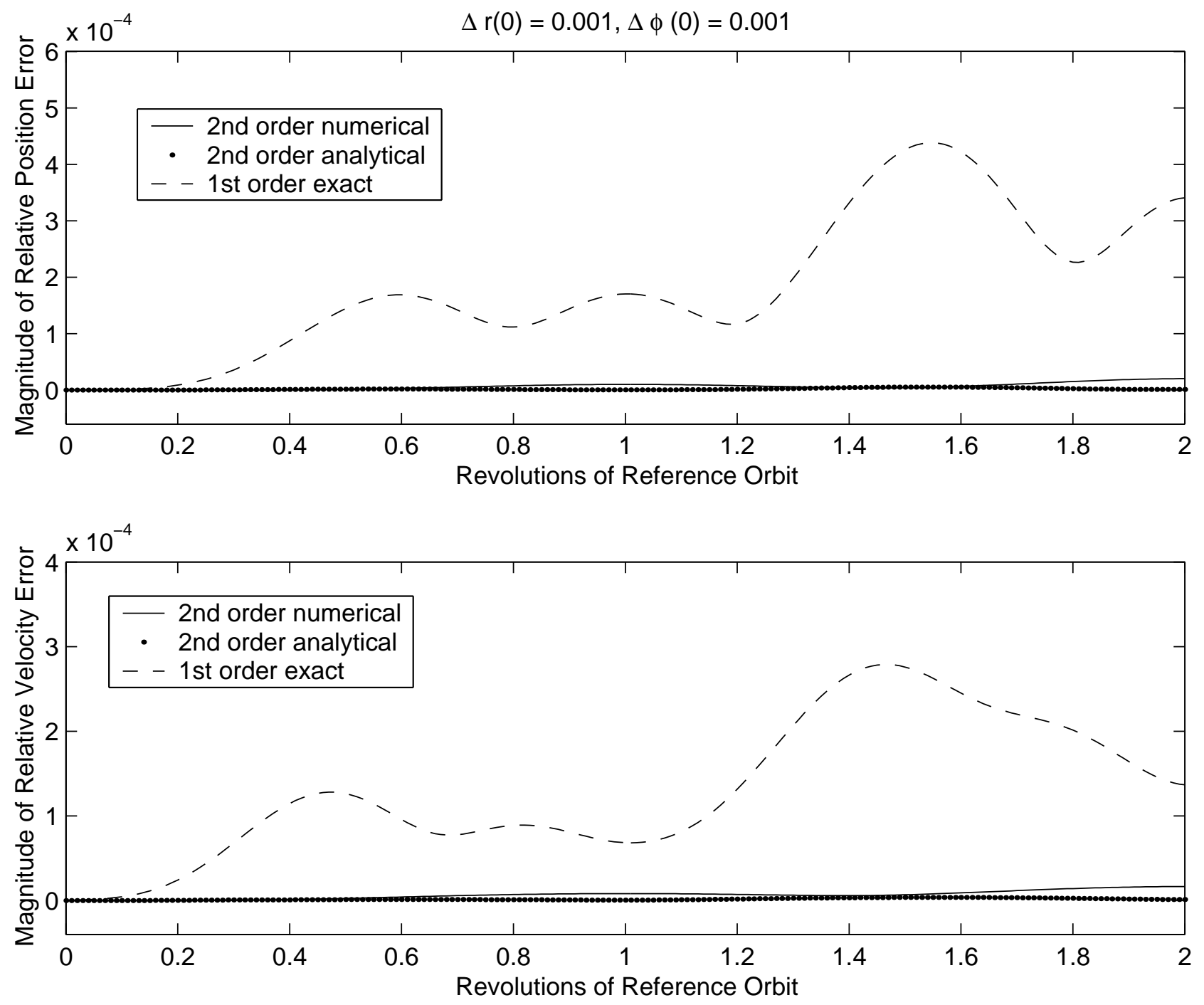

Figure C.18: Magnitude of Relative Position and Velocity Error for $\Delta \hat{r}(0)=0.001, \Delta \hat{\phi}(0)=$ 0.001 

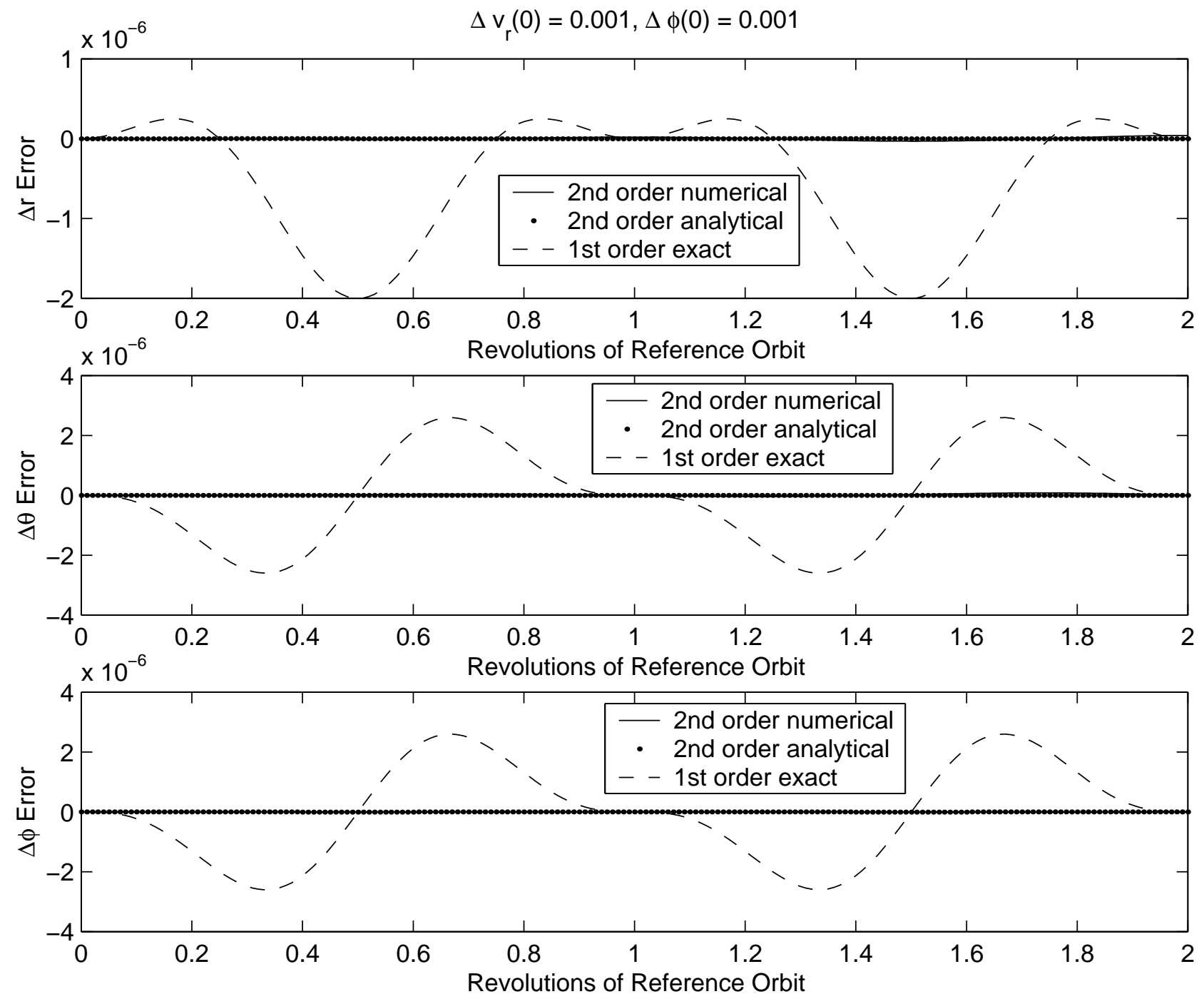

Figure C.19: Error of Position Variables for $\Delta \hat{v}_{r}(0)=0.001, \Delta \hat{\phi}(0)=0.001$ 

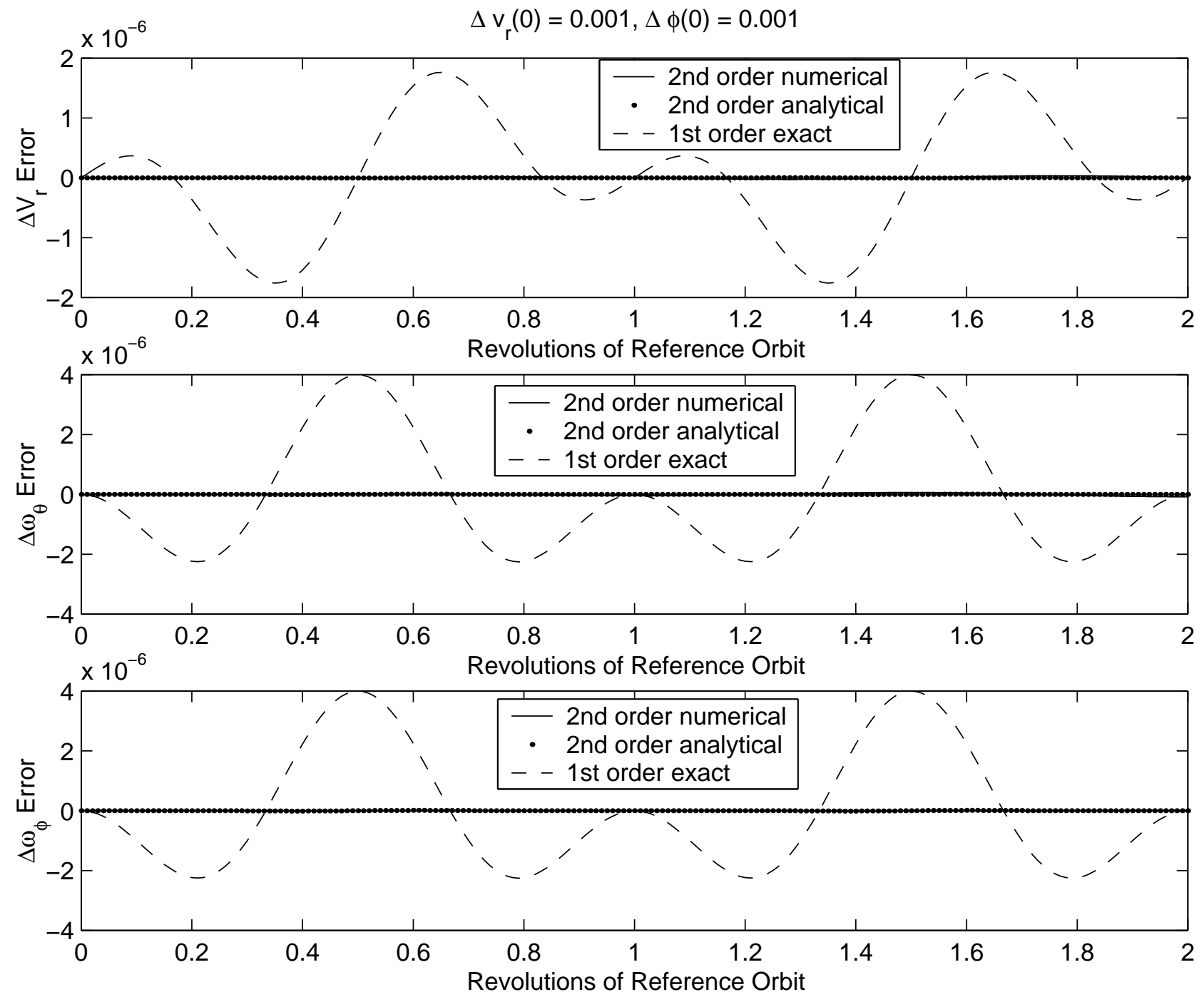

Figure C.20: Error of Velocity Variables for $\Delta \hat{v}_{r}(0)=0.001, \Delta \hat{\phi}(0)=0.001$ 

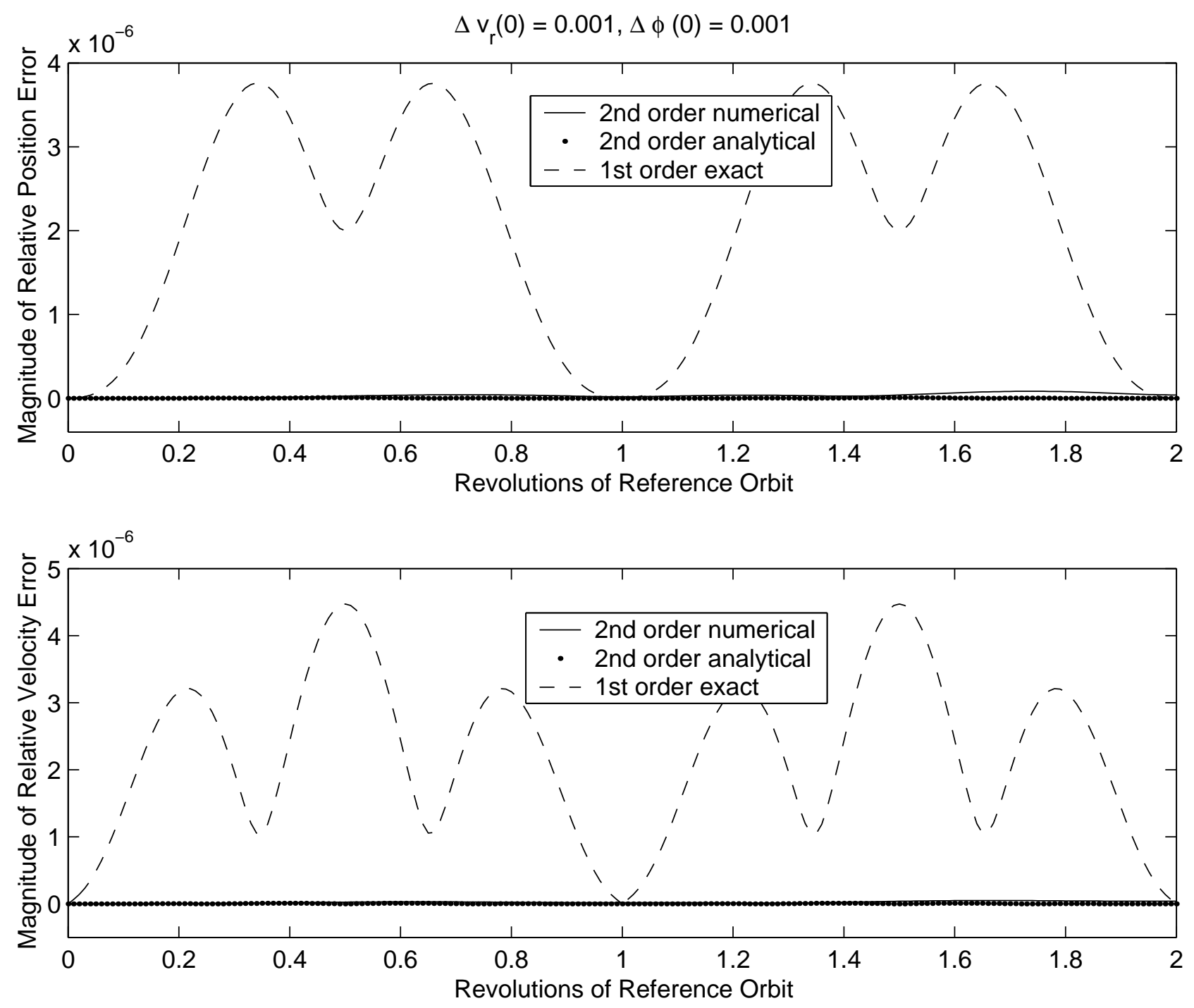

Figure C.21: Magnitude of Relative Position and Velocity Error for $\Delta \hat{v}_{r}(0)=0.001, \Delta \hat{\phi}(0)=$ 0.001 

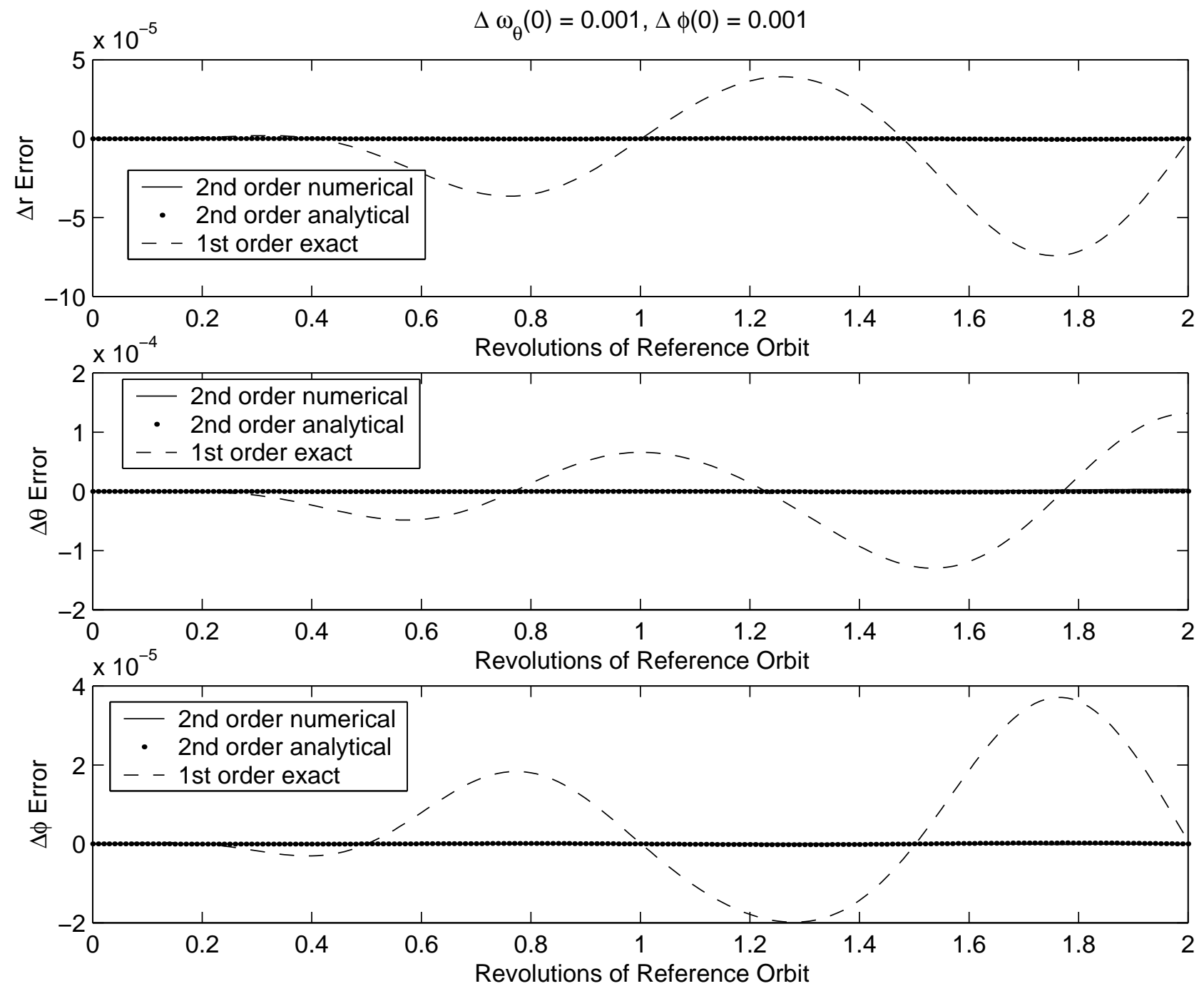

Figure C.22: Error of Position Variables for $\Delta \hat{\omega}_{\theta}(0)=0.001, \Delta \hat{\phi}(0)=0.001$ 

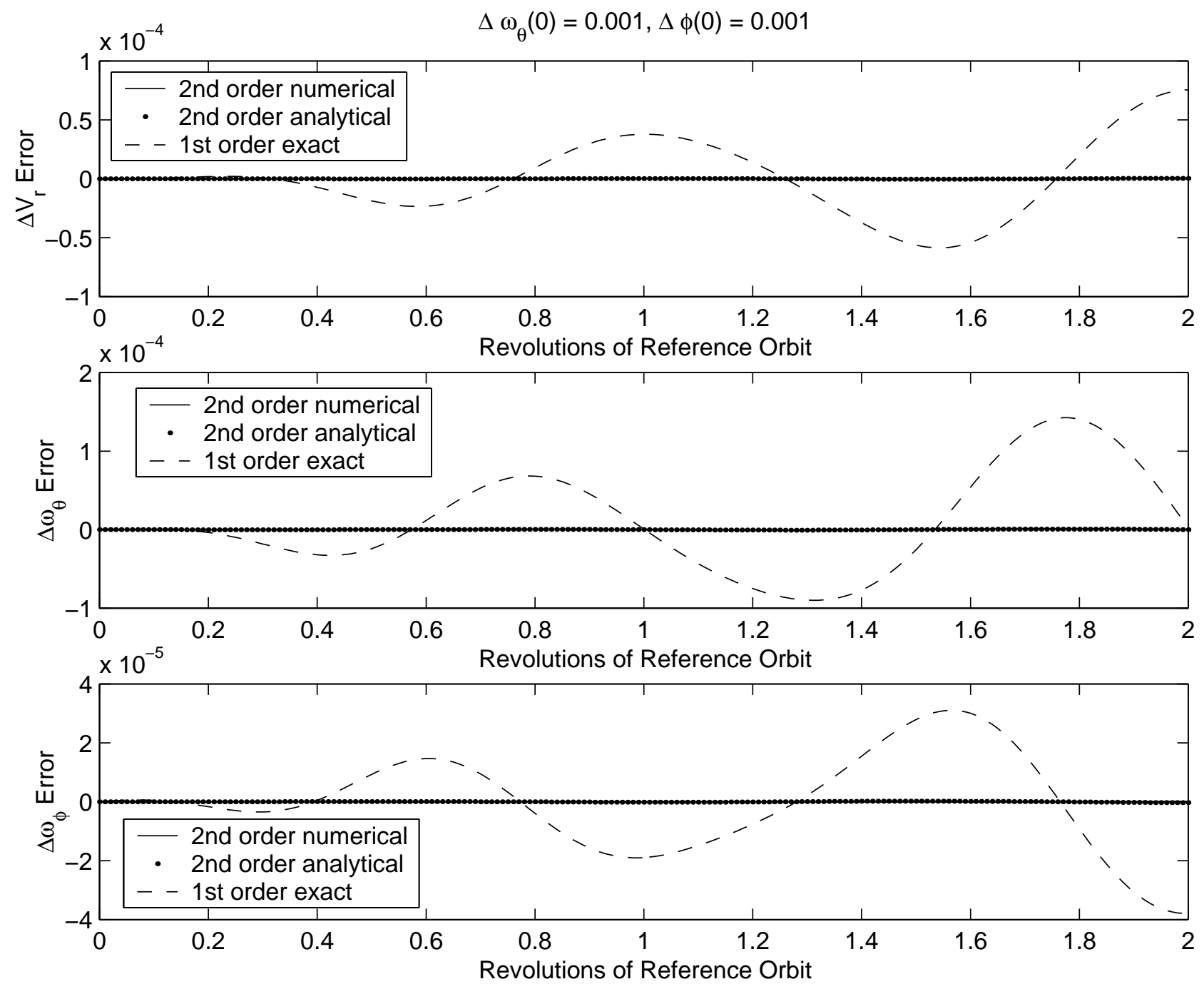

Figure C.23: Error of Velocity Variables for $\Delta \hat{\omega}_{\theta}(0)=0.001, \Delta \hat{\phi}(0)=0.001$ 

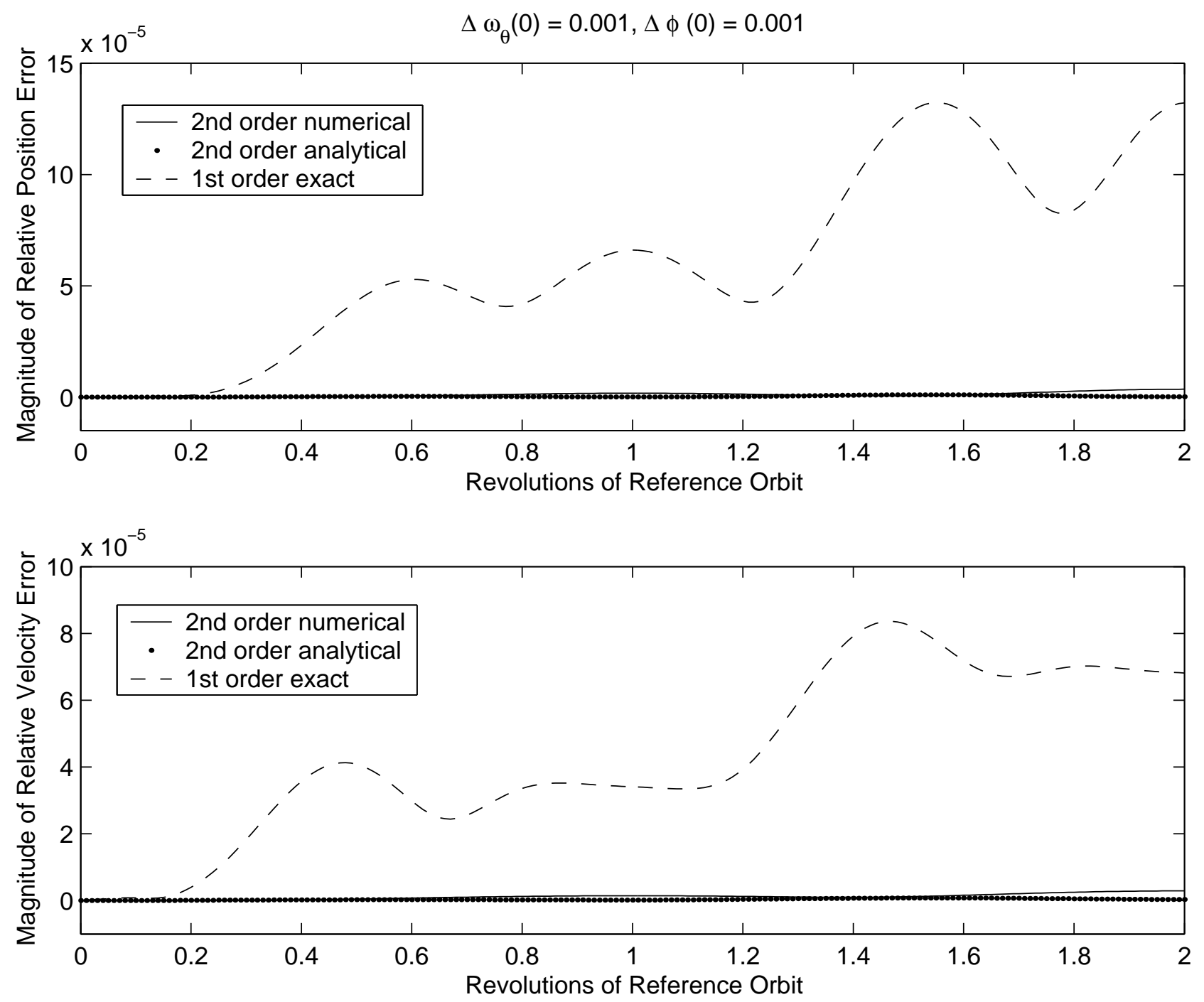

Figure C.24: Magnitude of Relative Position and Velocity Error for $\Delta \hat{\omega}_{\theta}(0)=0.001, \Delta \hat{\phi}(0)=$ 0.001 

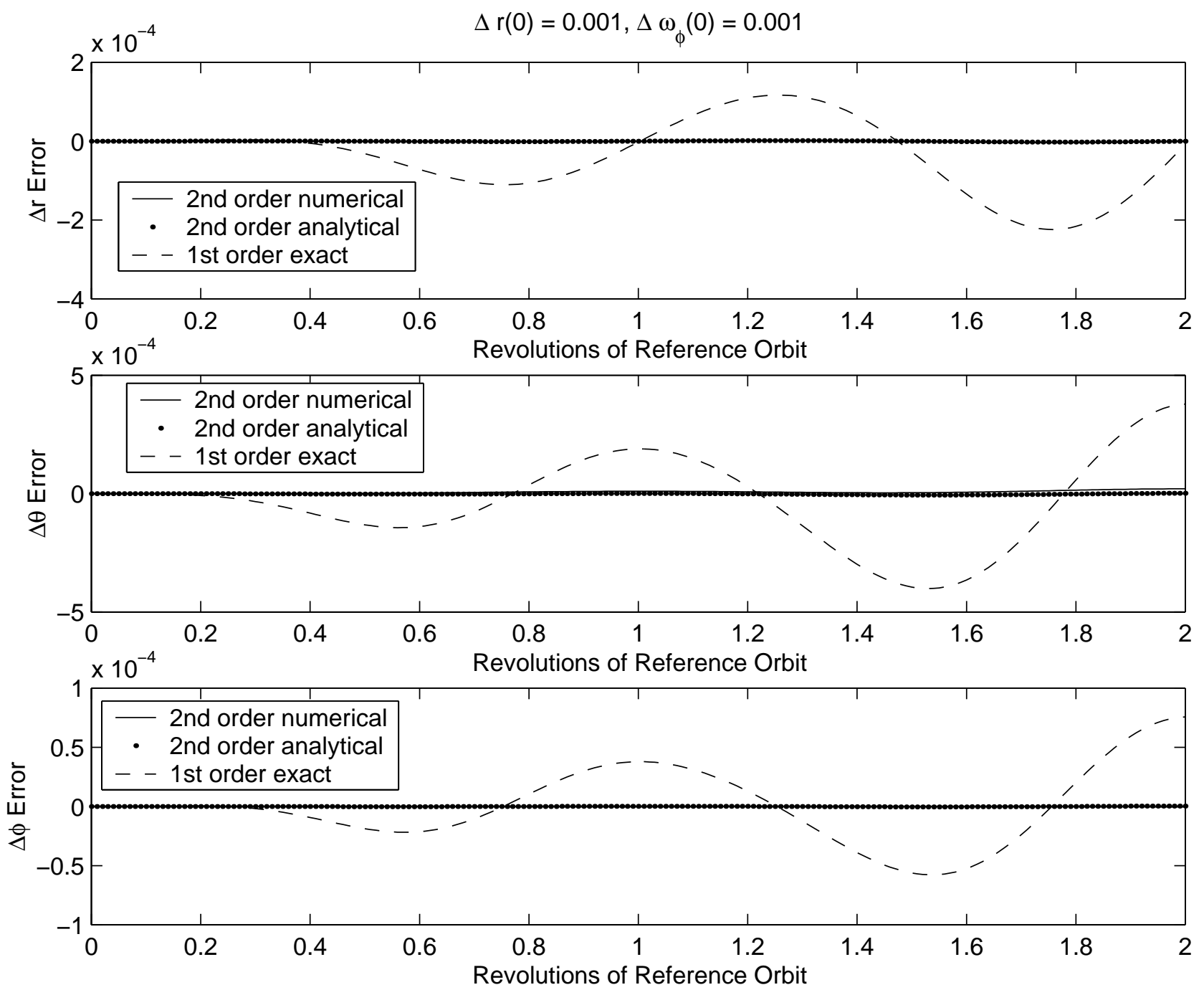

Figure C.25: Error of Position Variables for $\Delta \hat{r}(0)=0.001, \Delta \hat{\omega}_{\phi}(0)=0.001$ 

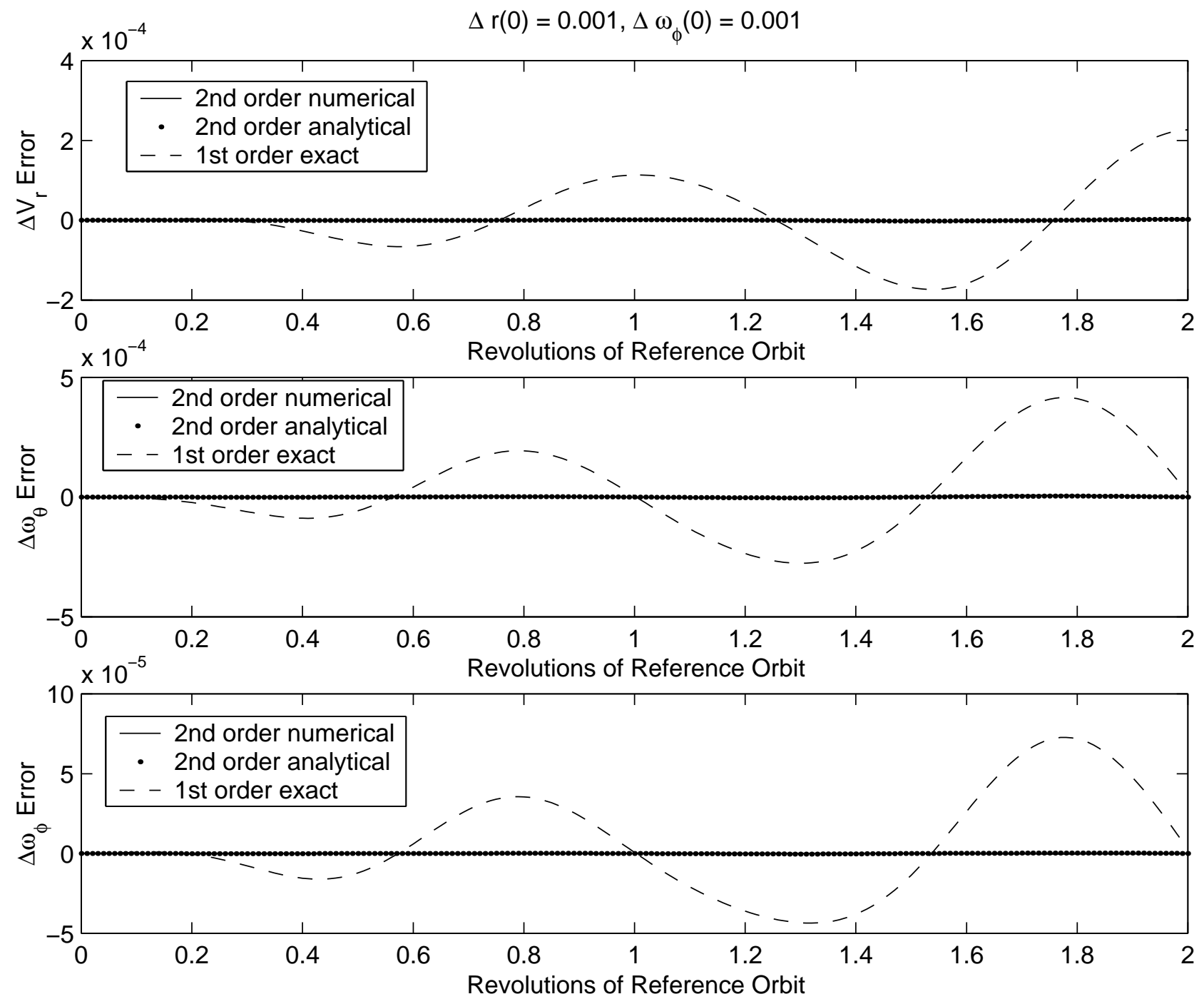

Figure C.26: Error of Velocity Variables for $\Delta \hat{r}(0)=0.001, \Delta \hat{\omega}_{\phi}(0)=0.001$ 

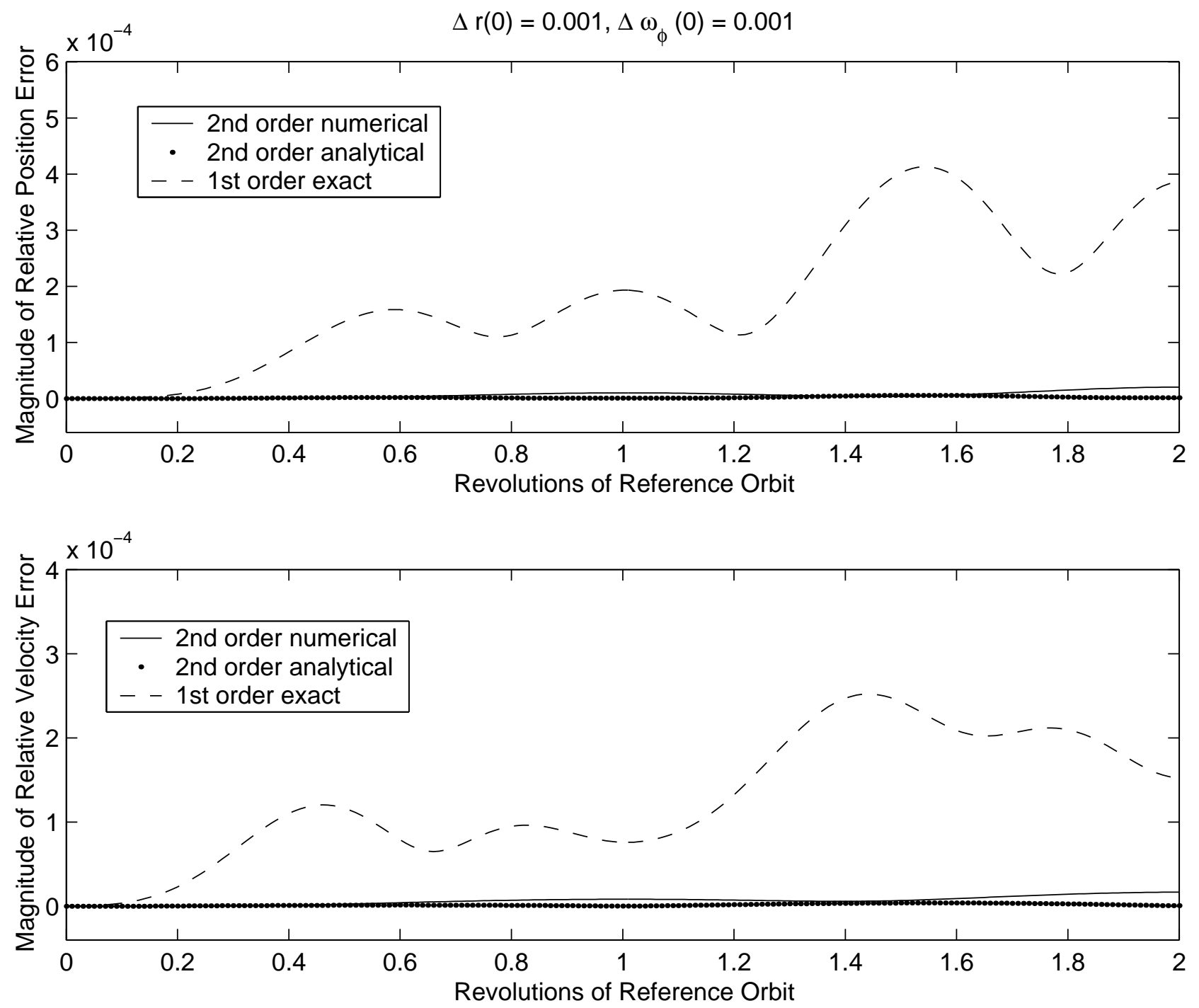

Figure C.27: Magnitude of Relative Position and Velocity Error for $\Delta \hat{r}(0)=0.001, \Delta \hat{\omega}_{\phi}(0)=$ 0.001 

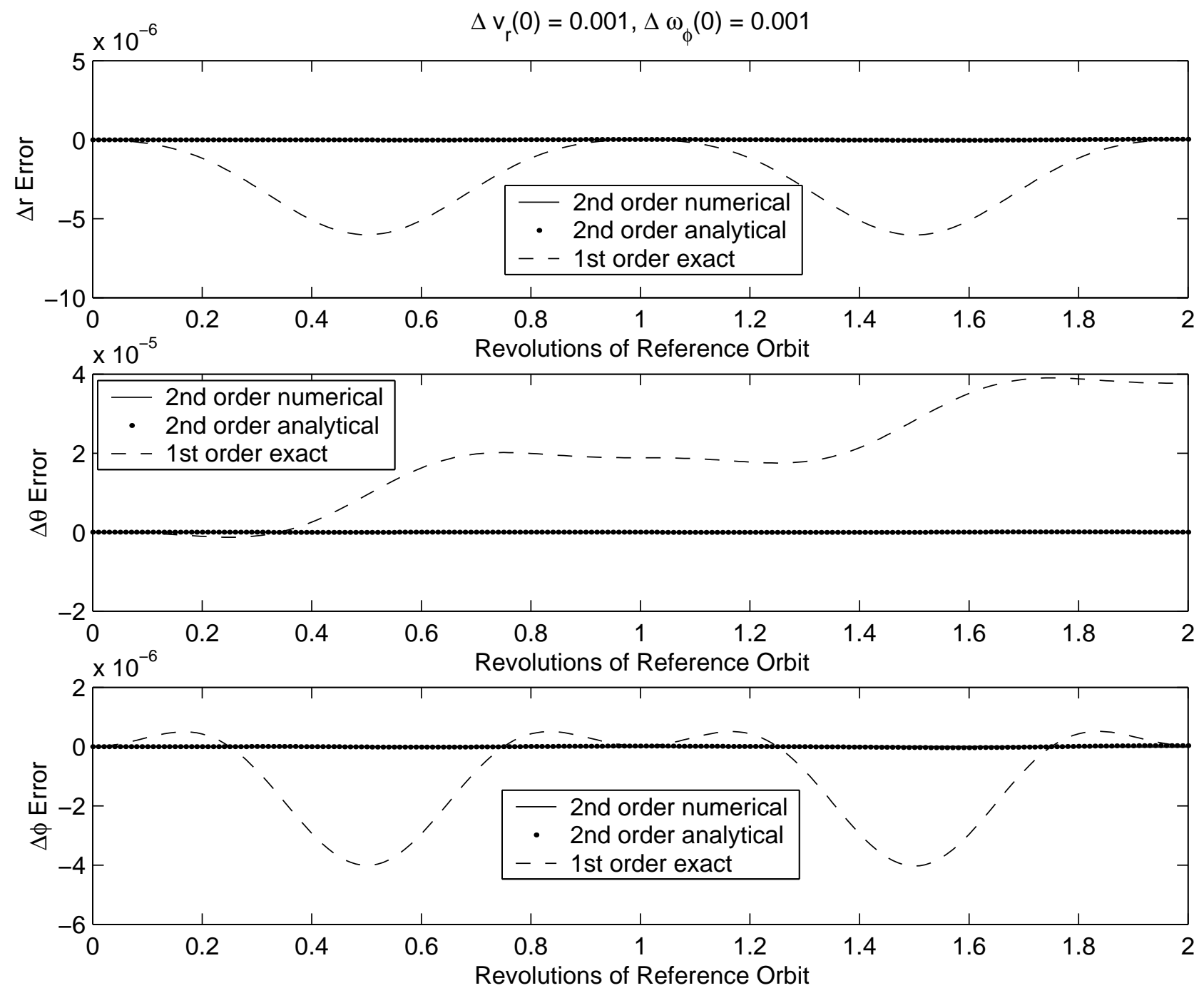

Figure C.28: Error of Position Variables for $\Delta \hat{v}_{r}(0)=0.001, \Delta \hat{\omega}_{\phi}(0)=0.001$ 

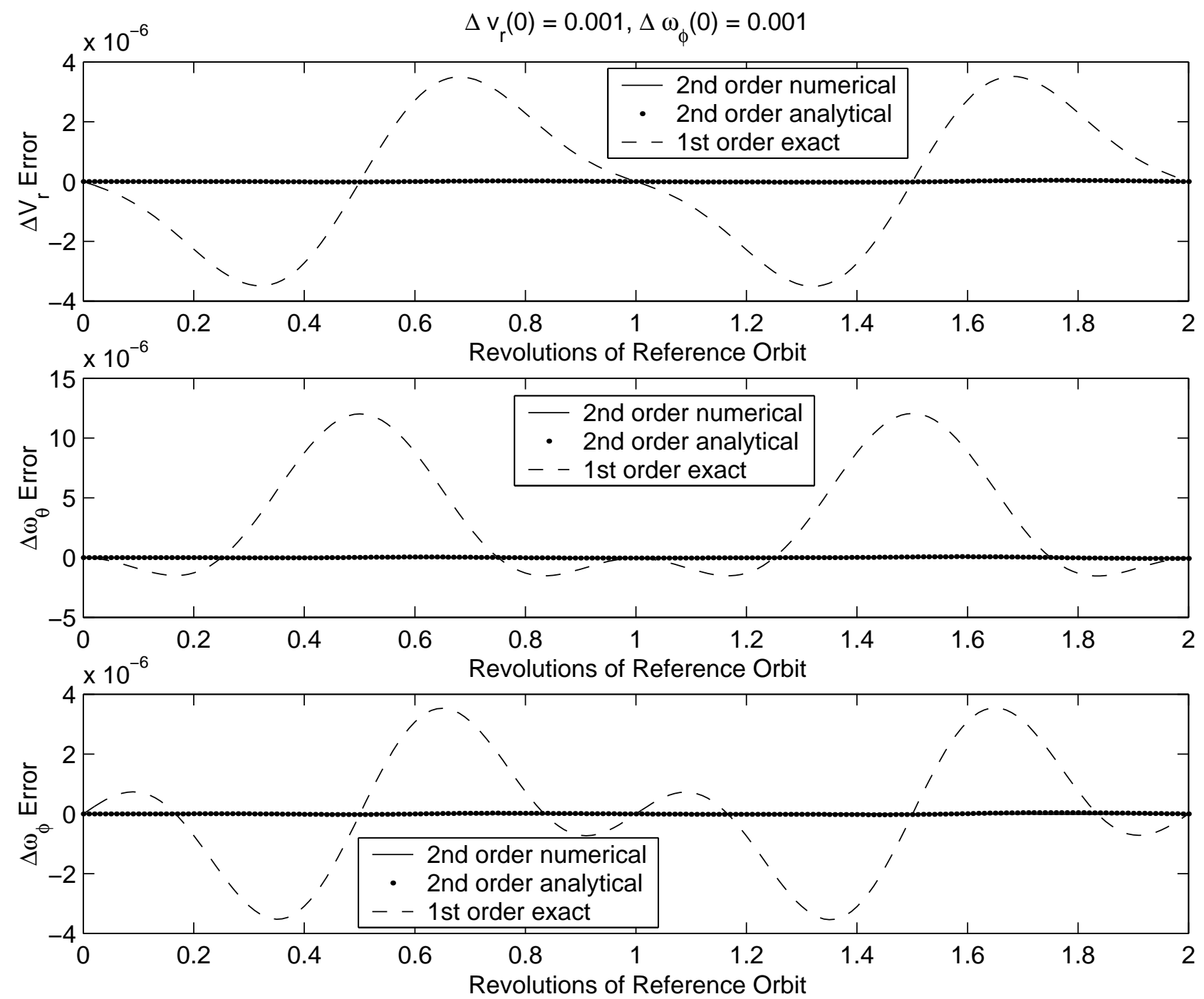

Figure C.29: Error of Velocity Variables for $\Delta \hat{v}_{r}(0)=0.001, \Delta \hat{\omega}_{\phi}(0)=0.001$ 

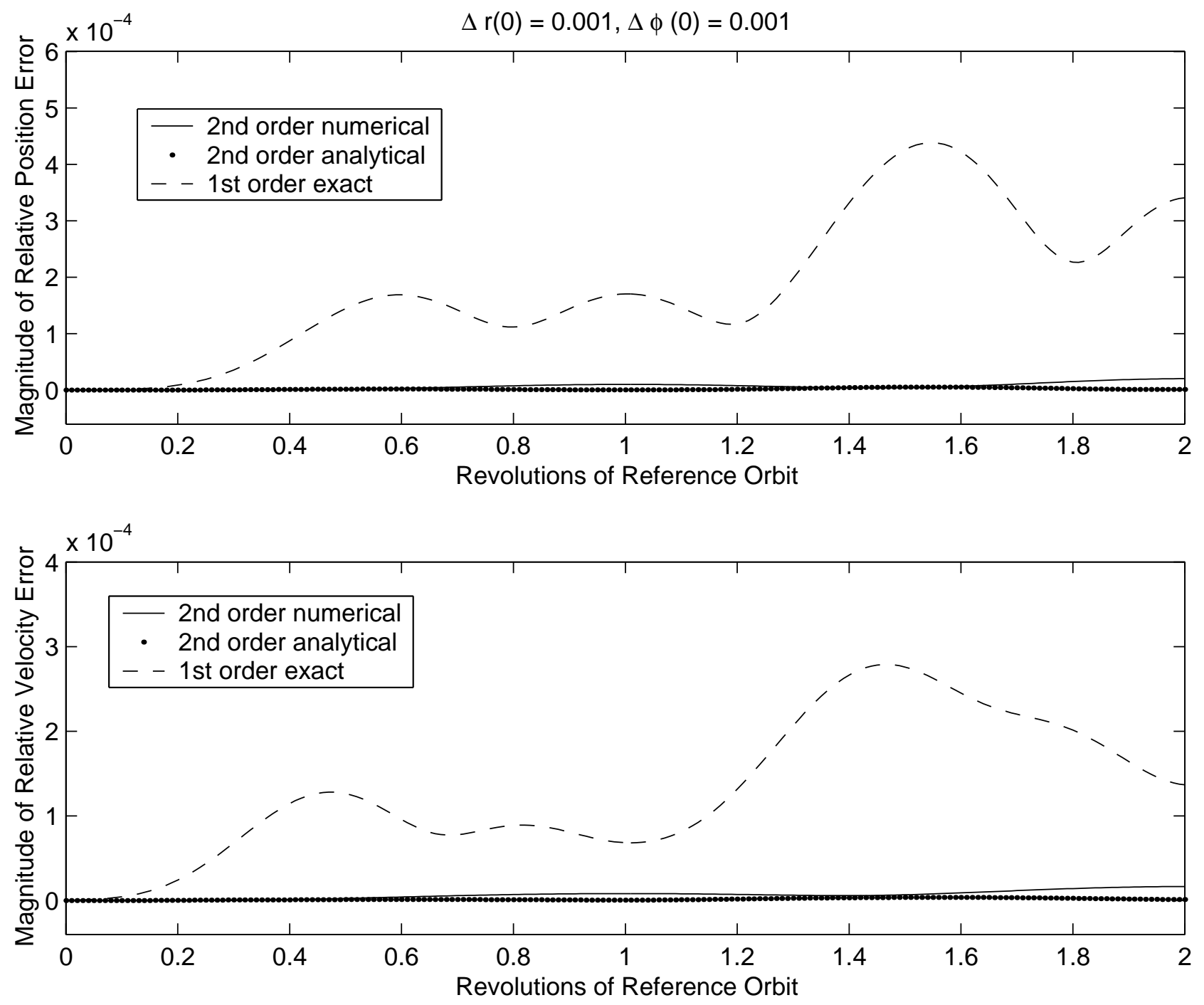

Figure C.30: Magnitude of Relative Position and Velocity Error for $\Delta \hat{v}_{r}(0)=0.001, \Delta \hat{\omega}_{\phi}(0)=$ 0.001 

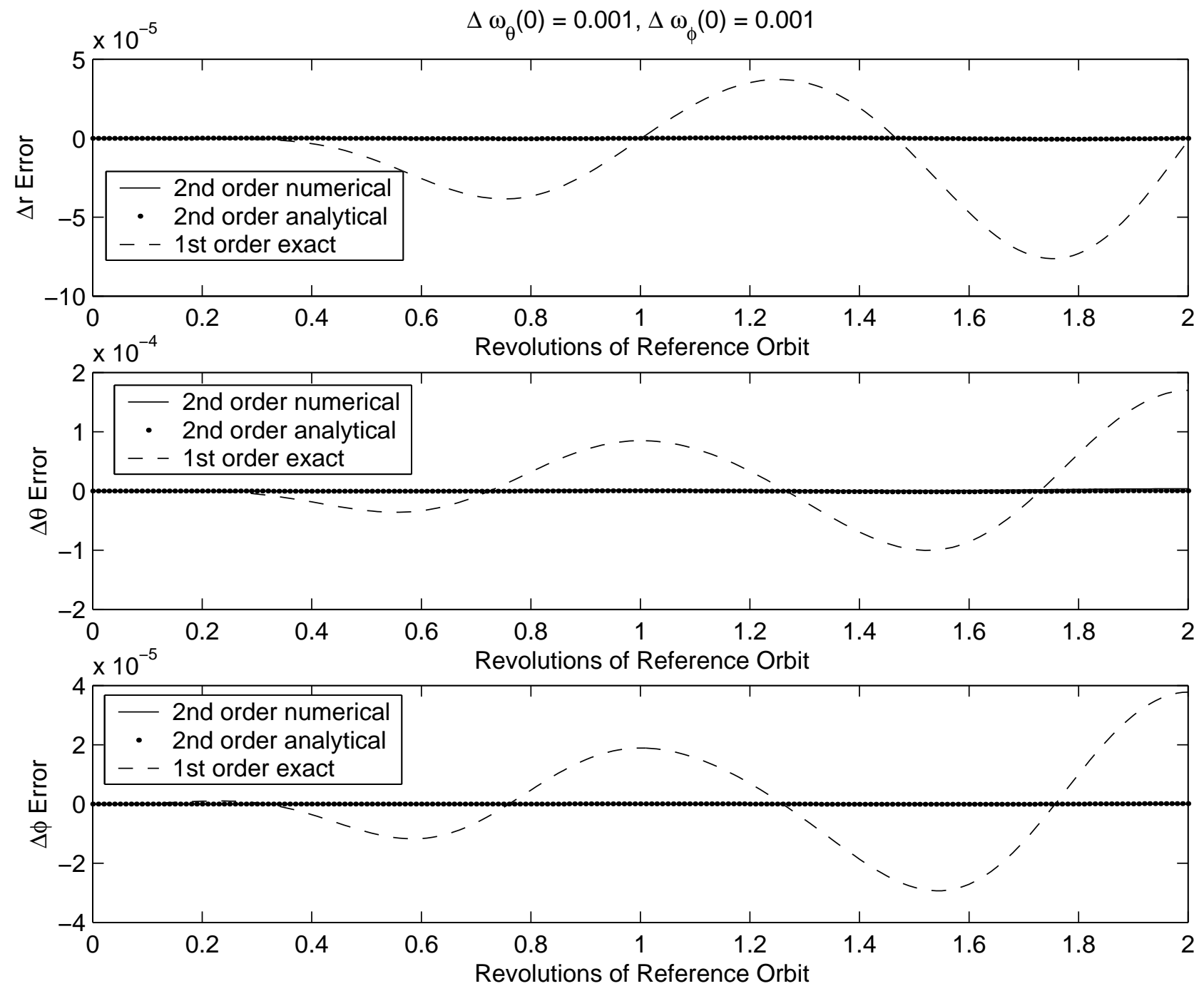

Figure C.31: Error of Position Variables for $\Delta \hat{\omega}_{\theta}(0)=0.001, \Delta \hat{\omega}_{\phi}(0)=0.001$ 

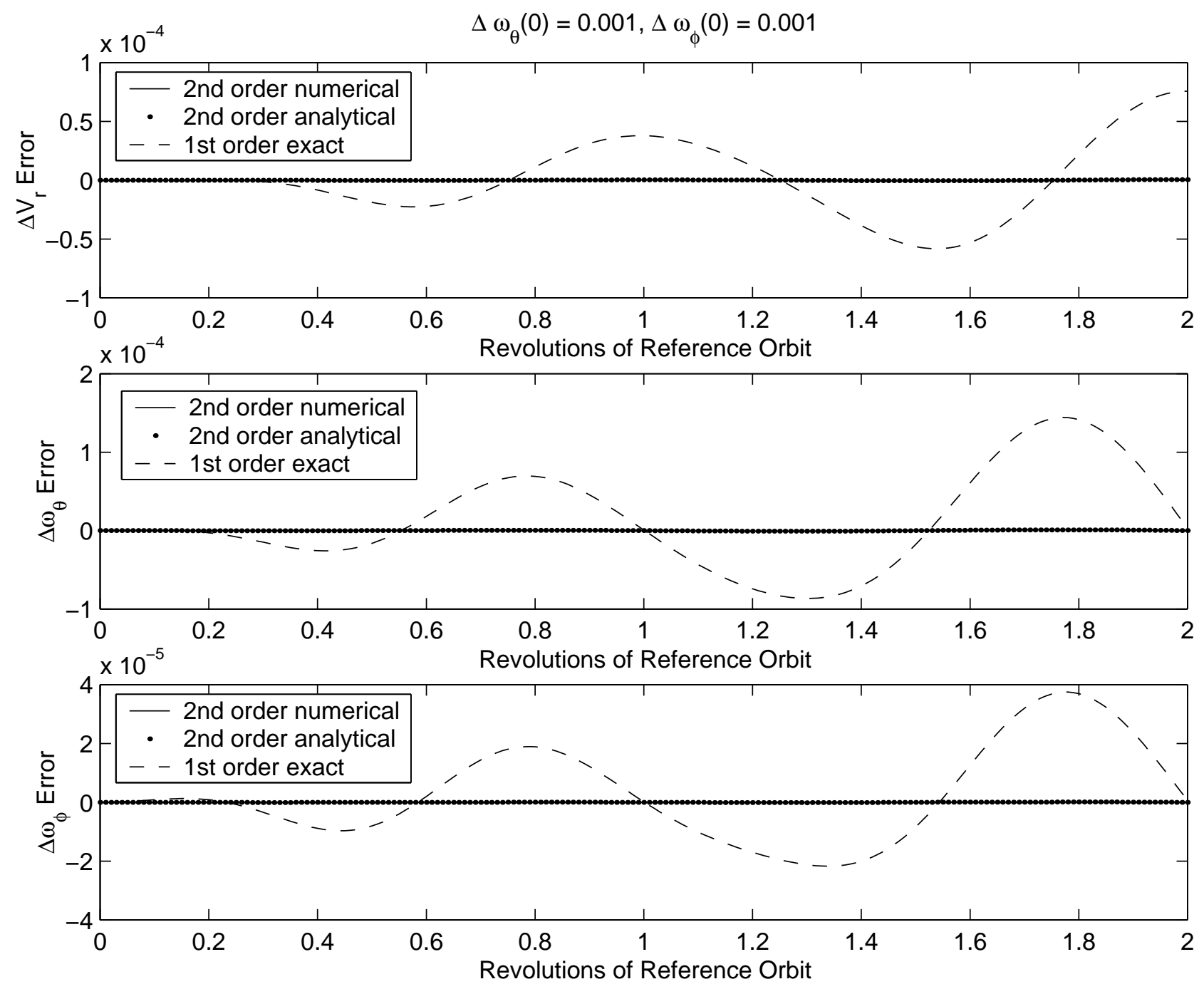

Figure C.32: Error of Velocity Variables for $\Delta \hat{\omega}_{\theta}(0)=0.001, \Delta \hat{\omega}_{\phi}(0)=0.001$ 

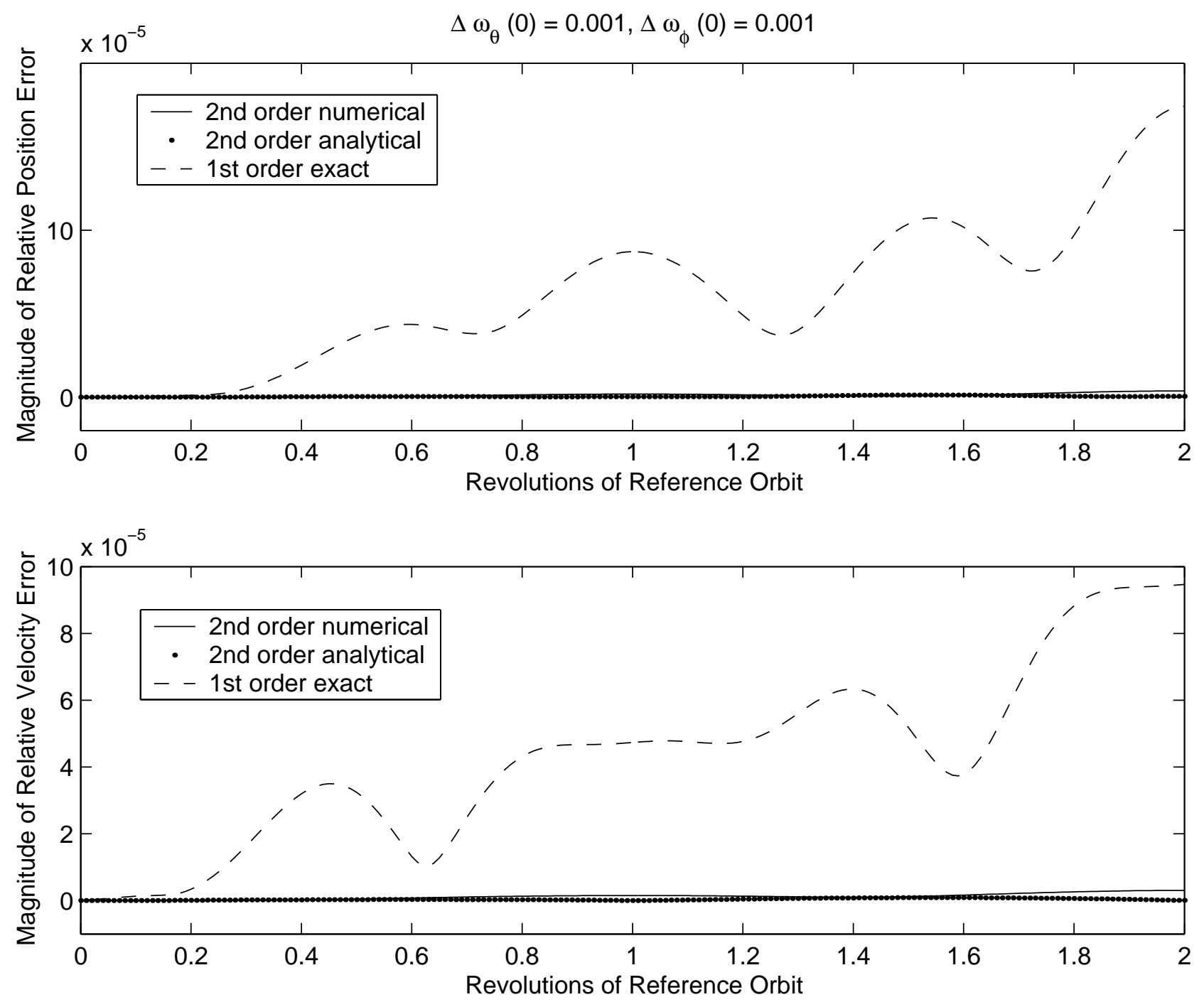

Figure C.33: Magnitude of Relative Position and Velocity Error for $\Delta \hat{\omega}_{\theta}(0)=0.001, \Delta \hat{\omega}_{\phi}(0)=$ 0.001 


\section{BIBLIOGRAPHY}

[1] Hill, G. W., "Researches in the Lunar Theory," American Journal of Mathematics, Vol. 1, No. 1, 1878, pp. 5-26, 129-147, 245-260.

[2] Clohessy, W. H. and Wiltshire, R. S., "Terminal Guidance System for Satellite Rendezvous," Journal of the Aerospace Sciences, Vol. 27, No. 5, 1960, pp. 653-658, 674.

[3] Anthony, M. L. and Sasaki, F. T., "Rendezvous Problem for Nearly Circular Orbits," AIAA Journal, Vol. 3, No. 7, 1965, pp. 1666-1673.

[4] Werlwas, R. W., A New First Order Solution to the Relative Motion Problem with Application to Intercept and Rendezvous, $\mathrm{PhD}$ thesis, Virginia Polytechnic Institute and State University, Blacksburg, VA, 1968.

[5] Jezewski, D. J. and Donaldson, J. D., "An Analytic Approach to Optimal Rendezvous Using Clohessy-Wiltshire Equations," Journal of the Astronautical Sciences, Vol. 27, No. 3, 1979, pp. 293-310.

[6] Kelly, T. J., "An Analytical Approach to the Two-Impulse Optimal Rendezvous Problem," Advances in the Astronautical Sciences, Vol. 87, No. 1, 1994, pp. 337-347.

[7] Lutze, F. H., "Unaided EVA Intercept and Rendezvous Charts," Journal of Spacecraft, Vol. 16, No. 6, 1979, pp. 426-431.

[8] Sabol, C., Burns, R., and McLaughlin, C. A., "Satellite Formation Flying Design and Evolution," Advances in the Astronautical Sciences, Vol. 102, No. 1, 1999, pp. 265-284.

[9] Sabol, C., Burns, R., and McLaughlin, C. A., "Satellite Formation Flying Design and Evolution," Journal of Spacecraft and Rockets, Vol. 38, No. 2, 2001, pp. 270-278.

[10] Chao, C. C., Pollard, J. E., and Janson, S. W., "Dynamics and Control of Cluster Orbits for Distributed Space Missions," Advances in the Astronautical Sciences, Vol. 102, No. 1, 1999, pp. 355-374.

[11] Hughes, S. P. Formation Flying Performance Measures for Earth-Pointing Missions. Master's thesis, Virginia Polytechnic Institute and State University, Blacksburg, VA, 1999.

[12] Hughes, S. P. and Hall, C. D., "Optimal Configurations for Rotating Spacecraft Formations," Journal of the Astronautical Sciences, Vol. 48, No. 2 and 3, 2000, pp. 225-247.

[13] Badesha, S. S., Heyler, G. A., Sharer, P. J., and Strikwerda, T. E., "Development of Formation Deployment and Initialization Concepts," In Flight Mechanics Symposium, Goddard Space Flight Center, May 1999. 
[14] Chichka, D. F., "Satellite Clusters with Constant Apparent Distribution," Journal of Guidance, Control and Dynamics, Vol. 24, No. 1, 2001, pp. 117-122.

[15] Vadali, S. R., Vaddi, S. S., and Alfriend, K. T., "New Concept for Controlling Formation Flying Satellites," In Space Flight Mechanics Conference, Santa Barbara, California, February 2001.

[16] London, H. S., "Second Approximation to the Solution of the Rendezvous Equations," AIAA Journal, Vol. 1, No. 7, 1963, pp. 1691-1693.

[17] DeVries, J. P., "Elliptic Elements in Terms of Small Increments of Position and Velocity Components," AIAA Journal, Vol. 1, No. 11, 1963, pp. 2626-2629.

[18] Euler, E. A. and Shulman, Y., "Second-Order Solution to the Elliptical Rendezvous Problem," AIAA Journal, Vol. 5, No. 5, 1967, pp. 1033-1035.

[19] Lancaster, E. R., "Relative Motion of Two Particles in Elliptic Orbits," AIAA Journal, Vol. 8, No. 10, 1967, pp. 1878-1879.

[20] Berreen, T. F. and Crisp, J. D. C., "An Exact and a New First-Order Solution for the Relative Trajectories of a Probe Ejected From a Space Station," Celestial Mechanics, Vol. 13, 1976, pp. 75-88.

[21] Szebehely, V., "Time Transformations for Relative Motion," Celestial Mechanics, Vol. 13, 1976, pp. 465-470.

[22] Garrison, J. L., Gardner, T. G., and Axelrad, P., "Relative Motion in Highly Elliptical Orbits," Advances in the Astronautical Sciences, Vol. 89, No. 2, 1995, pp. 1359-1376.

[23] Melton, R. G., "Relative Motion of Satellites in Elliptical Orbits," Advances in the Astronautical Sciences, Vol. 97, No. 2, 1997, pp. 2075-2094.

[24] Melton, R. G., "Time Explicit Representation of Relative Motion Between Elliptical Orbits," Journal of Guidance, Control and Dynamics, Vol. 23, No. 4, 2000, pp. 604-610.

[25] Vallado, D. A., Fundamentals of Astrodynamics and Applications, McGraw-Hill, New York, NY, 1997.

[26] Nayfeh, A., Introduction to Perturbation Techniques, Wiley, New York, NY, 1981.

[27] Holmes, M. H., Introduction to Perturbation Methods, Springer-Verlag, New York, NY, 1995.

[28] Kevorkian, J. and Cole, J. D., Multiple Scale and Singular Perturbation Methods, SpringerVerlag, New York, NY, 1996.

[29] Ogata, K., Modern Control Engineering, Prentice Hall, Upper Saddle River, NJ, 1997. 


\section{Vita}

The author was deployed on August 24th, 1976 in Alexandria, Virginia, but shortly thereafter he moved to Fairfax Virginia where he would live out those funny years in between birth and college. In the fall of 1994 he started his university career as a Physics and Astronomy major at the University of Maryland, but after a year he switched to Mathematics, and then after another semester (and a bureaucratic nightmare) he was able to add Aerospace Engineering as well. In the summer of 1999 was he awarded a B.S. in Aerospace Engineering and a B.S. in Mathematics. During his time at UMD, he worked part time for 2 years in the Astronomy department helping out with the archiving and distribution of comet data. He also spent a summer with the Visiting Student Enrichment Program at NASA/GSFC in the Scientific Computing Branch working on the modeling and simulation of volcanic plumes. For two summers he was a co-op in the Mathematics and Orbit Dynamics section of the Naval Research Lab where he worked on software for orbit determination and orbit prediction. In the fall of 1999 he came to Virginia Tech to work on a master's degree (hence the reason for writing this thesis). He is currently employed as a Project Engineer with Analytical Mechanics Associates, Inc. in Hampton, Virginia. 